\title{
Selbstorganisation magnetischer Nanopartikel auf facettierten Saphir-Substraten
}

\author{
Dissertation \\ zur Erlangung des Doktorgrades \\ der Mathematisch-Naturwissenschaftlichen Fakultäten \\ der Georg-August-Universität zu Göttingen \\ vorgelegt von \\ Carsten Herweg \\ aus Opladen jetzt Leverkusen \\ Göttingen 2005
}


Referent: Prof. Dr. H. C. Freyhardt

Korreferent: Prof. Dr. H. Hofsäß

Tag der mündlichen Prüfung: 18. März 2005 


\section{Inhaltsverzeichnis}

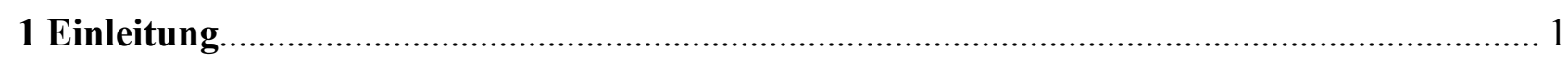

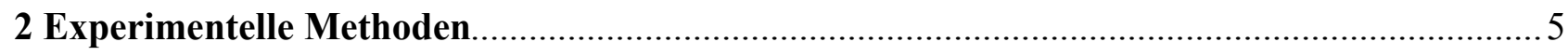

2.1 Deposition der Proben durch Trioden-Magnetron-Sputtern im UHV]..................................... 5

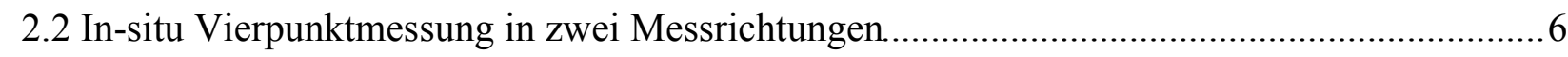

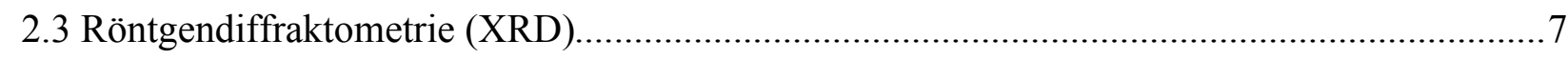

2.4 Rasterelektronenmikroskopie (SEM). ..................................................................................

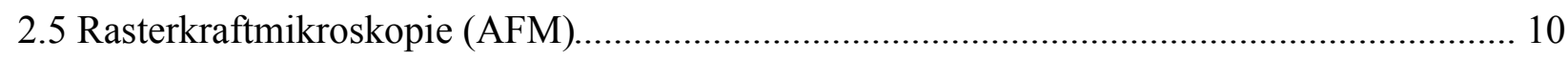

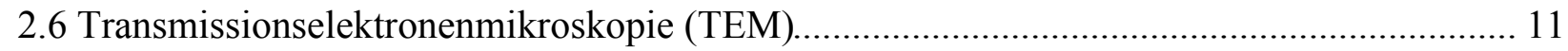

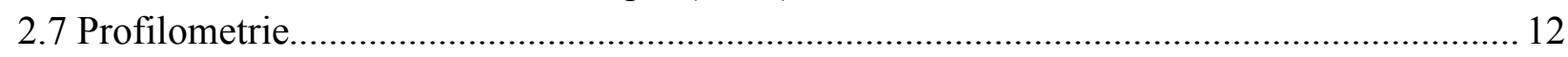

2.8 Das Feldionenmikroskop (FIM) und die tomographische Atomsonde (TAP). .......................12

2.9 Magnetisierungsverhalten mittels magneto-optischen Kerr-Effekts (MOKE), ...................... 13

3 Thermische induzierte Veränderungen von m-plane Saphir ............................................ 15

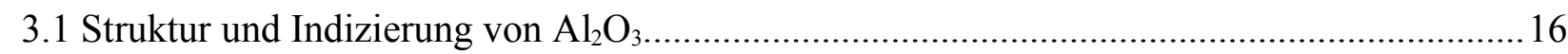

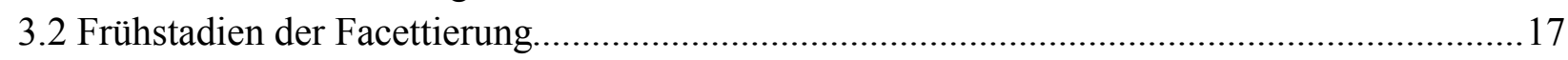

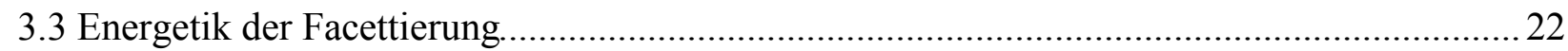

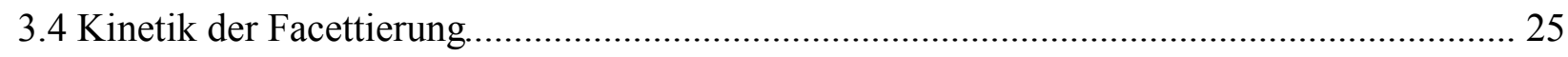

4 Transfer der Periodizität in Felder von Nanodrähten. ....................................................... 35

4.1 Nanodrähte durch Schattendeposition,............................................................................ 35

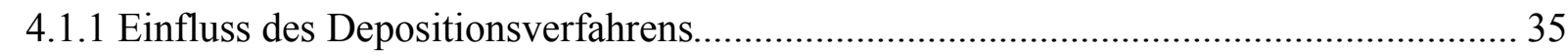

4.1.2 Inselwachstum und das Überwachsen der Facettenkämme am Beispielsystem Gold.....36

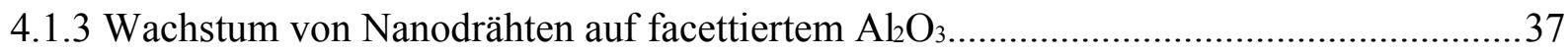

4.1.4 Einfluss von Materialmenge und -verteilung am Beispiel Kobalt.................................. 46

4.2 Gestaltumwandlung durch Temperaturbehandlung. .......................................................... 51

4.2.1 Stadien der Gestaltumwandlung am Beispiel der Eisennanodrähte. ................................51

4.2.2 Lokales und globales Magnetisierungsverhalten von linear angeordneten Eisenpartikeln.

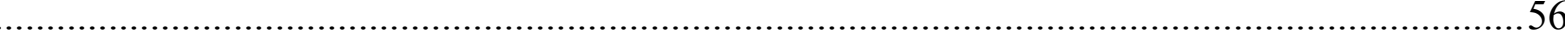

4.2.3 Einfluss von Materialmenge und -verteilung am Beispiel Kobalt ................................. 62

5 Transfer der Periodizität in Vielfachschichtsysteme. ............................................................ 71

5.1 Deposition von Vielfachschichtsystemen auf facettierte Substrate. ..................................... 71

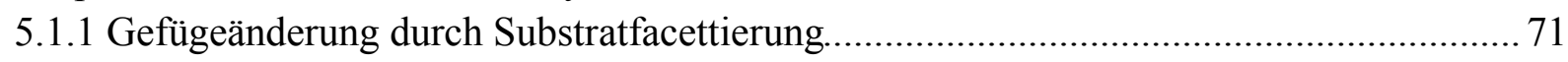

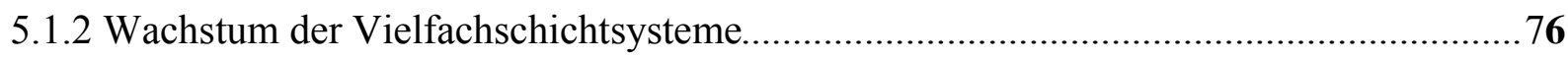

5.1.3 Innerer Aufbau und Periodizität der Vielfachschichtsysteme. ........................................... 86

5.1.4 Texturanalyse der Vielfachschichtsysteme.......................................................... 91

5.1.5 Einfluss der Substratfacettierung und der Facettenoberfläche auf die Mikrostruktur der

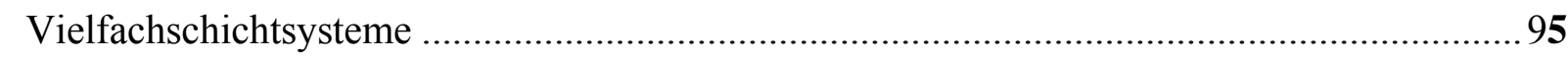

5.2 Gestaltumwandlung durch Temperaturbehandlung ......................................................... 109

5.2.1 Thermische Charakteristik der Gestaltumwandlung. ................................................. 109

5.2 .2 Veränderungen von Gefüge und Mikrostruktur....................................................... 121

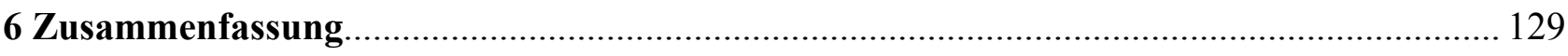

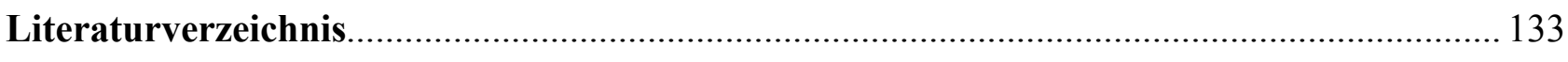





\section{Einleitung}

Selbstorganisationseffekte beeinflussen und bestimmen den Aufbau in der belebten, aber auch unbelebten Natur in allen Größenordnungen, vom atomaren Aufbau bis hin zu globalen Dimensionen bei Wettersystemen [Gol 02], [Bir 03], [Kes 03], [Whi 02]. Die Gründe, sich mit Selbstorganisationseffekten $\mathrm{zu}$ beschäftigen, sind vielfältig. Primär ist es wahrscheinlich die Faszination, die das Auftreten von spontaner Ordnung aus dem Regellosen heraus auf den Menschen ausübt, die auch den Wunsch nach einem besseren Verständnis entstehen lässt. Darüber hinaus ist Selbstorganisation das Bauprinzip der belebten Natur und daher bedeuten Einblicke in dieses Bauprinzip gleichzeitig ein tieferes Verständnis von Leben.

Beim Aufbau makroskopischer Formen aus nanoskopischen Bauteilen ist die Selbstorganisation eine der wenigen praktikablen Strategien. Da der Ansatz Zeit- und Kosteneffizienz verspricht, gewinnt er im Bereich der Nanotechnologie immer mehr an Bedeutung. Sequentielle Verfahren wie die manipulativen Rastersondenverfahren bieten zwar heutzutage die Möglichkeit zur gezielten Anordnung von Atomen auf Oberflächen mit höchster Genauigkeit, doch trotz vieler Versuche zur Parallelisierung dieser Prozesse scheint die Kluft zwischen den atomaren Dimensionen der Bausteine und den makroskopischen Abmessungen der Zielprodukte von technischen Produktionsprozessen auf diese Weise nicht zu schließen zu sein. Selbstorganisierte Prozesse können dieses Problem prinzipiell beheben. Da die Produkte aber in der Regel nicht von vornherein alle gewünschten Eigenschaften bieten, ist eine Beeinflussung notwendig, um diese zu erzielen. Ein wichtiges Anwendungsgebiet für Nanostrukturierung stellt die Informations- und Speichertechnologie dar. Diese verlangt nach immer höheren Speicherdichten und damit kleineren Abmessungen einzelner magnetischer Bereiche. Schon heute werden bei Festplatten höchster Schreibdichte die magnetischen Speicherschichten in Segmenten $<100 \mathrm{~nm}$ strukturiert (z.B. durch Ionenbeschuss, „patterned media“ [Sch 04]), um die Wechselwirkung magnetischer Domänen zu mindern und somit Speicherbereiche zu verkleinern und dichter anordnen zu können.

Die selbstorganisierte Anordnung magnetischer Nanopartikel ist ein eleganter, kostengünstiger und erfolgversprechender Weg, diese Strukturen zu erzeugen [Ros 01]. Dabei können Anordnungen von metallischen Nanopartikeln einerseits auf glatten Unterlagen erreicht werden. Eine Möglichkeit sind vergleichsweise aufwändige lithographische Verfahren, mit denen z.B. lineare Anordnungen von Gold-Nanopartikeln [Mai 02] oder Eisen- und Kobalt-Punktfelder mit Durchmessern der Einzelelemente von 600nm hergestellt wurden [Was 98]. Durch weitere Verbesserung der Verfahren, wie z.B. der Interferenzlithographie, lassen sich zwar Anordnungen in Distanzen und Abmessungen $<100 \mathrm{~nm}$ erreichen, jedoch steigt der präparative Aufwand und die Empfindlichkeit der Prozesse stark an [Ros 02].

In einem weiteren, die Selbstorganisation nutzenden Verfahren wurden metallische Partikel in Form von Micellen (von Co-Polymeren umgeben) auf glatte Substrate aufgebracht [Wie 01]. Die sterischen Eigenschaften der Polymere beeinflussen den Abstand der Partikel, die üblicherweise durch Plasmaveraschung nachträglich von den Polymerresten getrennt werden müssen. Dies führt oft zu erheblicher Oxidation der magnetischen Partikel [Kas 03]. Auch kombinierte Verfahren aus Elektronenstrahllithographie und selbstanordnenden organischen Molekülen konnten erfolgreich eingesetzt werden, um Bereichsgrößen $<30 \mathrm{~nm}$ zu erreichen [Hat 01]. 
Andererseits ist es möglich, das Substrat schon in die Nanostrukturierung mit einzubeziehen. Ein Beispiel ist die Laserablation durch eine geeignete Maskenoptik [Sim 96]. Mit diesem Verfahren konnten Gräben eines minimalen Abstands von 360nm präpariert werden. Mittels Deposition und nachfolgender Laserbestrahlung der so entstandenen metallischen Streifen konnten lineare Anordnungen von Cu-Partikeln eines Durchmessers von ca. 200nm erzielt werden [Che 97].

Eine weitaus elegantere Methode ist es dabei, das Substrat nicht nur in die Nanostrukturierung mit einzubeziehen, sondern bereits diese Veränderung der Morphologie der Unterlage durch einen Selbstorganisationseffekt zu erzielen. Entsprechende Ordnungsphänomene von Kristalloberflächen gibt es unter geeigneten Bedingungen bereits in großer Vielfalt [Tei 02]. So kommt es etwa zu Rekonstruktionen durch die fehlenden Bindungspartner der Oberflächenatome [Har 80]. Meyer et al. konnten damit ausgezeichnete Positionen der Gold(111)-Rekonstruktion als Nukleationszentren für Eiseninseln nutzen [Mey 96]. Weiterhin ist es durch geringen Fehlschnitt einer Oberfläche gegenüber einer niedrig indizierten Kristallfläche und anschließender, geeigneter Temperung möglich, periodische bzw. quasiperiodische Anordnungen von atomaren Stufen auf diesen so genannten vizinalen Oberflächen zu erzielen [Pim 98], [Pre 04]. Diese wurden vielfach durch Dekoration der Stufen zur Erzeugung von Drahtfeldern benutzt [Him 99]. In SiGe-Systemen konnten ferner durch Fehlpassungsspannungen schachbrettartige Versetzungsanordnungen erzeugt und Insel-Anordnungen auf diese Weise manipuliert werden [Tei 00]. Eine weitere Methode der selbstorganisierten Nanostrukturierung stellt der Effekt der Oberflächenfacettierung dar. Dabei werden z.B. auf $\alpha-\mathrm{Al}_{2} \mathrm{O}_{3}$-Kristallflächen [Hef 97], aber auch auf speziellen Flächen anderer Oxide und Halbleiter, wie z.B. Silizium [Son 97], spitzdachartige Oberflächenmorphologien im Nanometerbereich erzeugt.

Neben der Anordnung von Material auf der Oberfläche strukturierter Substrate durch gezielte Selbstorganisation sind in der Vergangenheit bei Halbleitern erfolgreich erste dreidimensionale Anordnungen auf vergleichbare Weise erzeugt worden. Hierzu wurden Schichtsysteme genutzt, bei denen durch die Kontrolle des Wachstumsverhaltens während der Depositon separierte Inseln, z.B. $\mathrm{Si}_{0,3} \mathrm{Ge}_{0,7}$-Inseln auf $\mathrm{Si}(100)$, gewachsen werden konnten [Ter 96]. Durch Spannungsvermittlung konnten dann an der glatten Oberfläche der folgenden Si-Zwischenschicht gezielt wieder $\mathrm{Si}_{0,3} \mathrm{Ge}_{0,7}$-Inseln positioniert werden. Durch dieses Verfahren wurden auch dreidimensionale PbSe[Spr 98] und CdZnSe-Inselanordnungen [Shc 99] in Halbleiter-Heterostrukturen möglich.

Für die Anordnung von Partikeln in metallischen Systemen erfordern veränderte elastische Konstanten und Wachstumsmoden andere Verfahrensweisen, die über eine reine Spannungs vermittlung hinausgehen. Möglichkeiten bieten hier Temperbehandlungen im Anschluss an die Deposition von Schichtsystemen nicht mischender Komponenten wie z.B. Niob/Kupfer [Tro 96], Eisen/Gold oder Eisen/Silber [Her 99]. Auf glatten Substraten wurde durch Tempern bei niedrigen Temperaturen ein Zerfall einzelner Lagen in separierte, sphärische Partikel beobachtet, wodurch die periodische Lagenstruktur zerstört wurde. So konnten z.B. in Schichtsystemen aus 50 Doppellagen der Kombination 10nm Gold und 2nm Eisen durch Temperung bei $330^{\circ} \mathrm{C}$ Eisenpartikel eines mittleren Durchmessers von ca. 10nm in einer Goldmatrix gebildet werden. Da die Schichten mittels Sputterdeposition hergestellt wurden, bilden sich typische Wachstumssäulen. Die mikrostrukturell defektreichen Bereiche der Wachstumssäulengrenzen konnten als bevorzugte Nukleationsbereiche der Eisenpartikel identifiziert werden, wodurch es zu einer perlenkettenartigen Anordnung der Partikel parallel zur Schichtnormalen kommt [Tro 00]. Durch Einstellung der Abstände dieser defektreichen Bereiche könnte somit auch Einfluss auf die Partikelanordnung genommen werden. 
Ziel dieser Arbeit ist es nun, einerseits zu untersuchen, inwieweit Selbstorganisationsmechanismen nutzbar sind, um mit technisch einfach realisierbaren Temperprozessen nanostrukturierte Substrate mit periodischer Anordnung von Facetten zu erzeugen. Andererseits ist zu untersuchen, wie sich diese Facetten auf das Wachstum von Metallfilmen und -schichtpaketen auswirken, ob sich die Position von Wachstumssäulen und deren Grenzen beeinflussen und dadurch eine gezielte Anordnung defektreicher Bereiche in den deponierten Filmen erzeugen lässt. Dies sollte es ermöglichen, sowohl auf den Oberflächen der Substrate als auch im Volumen der deponierten Filme magnetische Nanopartikel periodisch anzuordnen.

Da für die Selbstorganisation der deponierten Materialien ein Substrat benötigt wird, das auch bei den Temperprozessen der abgeschiedenen dünnen Schichten (bis $600^{\circ} \mathrm{C}$ ) form- und strukturstabil ist und nicht zur Reaktion mit den deponierten Materialien neigt, wird zur Nanostrukturierung der Substrate in dieser Arbeit die Oberflächenfacettierung von $(10 \overline{1} 0)-\mathrm{Al}_{2} \mathrm{O}_{3}$ verwendet. Kapitel 3 enthält dementsprechend die Analyse der Stadien der Facettierung, wodurch zum einen die erzielbaren morphologischen Merkmale genau charakterisiert, zum anderen ein Verständnis dieser Gestaltänderung erreicht werden soll. Da diese Gestaltänderung erst bei hohen Temperaturen $\left(>1300^{\circ} \mathrm{C}\right)$ erfolgt, steht damit für die weiteren Experimente ein inertes und thermisch stabiles, nanostrukturiertes Substrat zur Verfügung.

Auf diesen facettierten Oberflächen sollen zunächst unter geeigneten Bedingungen Nanodrähte durch Abscheidung magnetischer Materialien wie Eisen und Kobalt auf einer der Facettenseiten erzeugt werden (Kapitel 4).

Für die Analyse der Stadien der Oberflächenveränderung der Substrate, wie auch bei den Untersuchungen zum Wachstum mittels Sputterns deponierter Metalle auf den Facettenseiten, wird mit der Rasterelektronenmikroskopie (SEM), der Rasterkraftmikroskopie (AFM) und der magnetischen Rasterkraftmikroskopie (MFM) auf komplementäre, oberflächensensitive Verfahren zurückgegriffen.

In einem weiteren Selbstorganisationsschritt sollen die so erzeugten Nanodrähte durch einen einfachen, thermischen Prozess in linear angeordnete, magnetische Partikel umgewandelt werden. Neben der Charakterisierung des gesamtmagnetischen Verhaltens mittels des magnetooptischen Kerr-Effekts (MOKE), sollen in Kombination mit MFM-Messungen ortsaufgelöste Analysen zum Magnetismus und zum möglichen Kopplungsverhalten der Drähte und der daraus erzeugten Partikel durchgeführt werden. Die Bedingungen für den thermischen Zerfall der Drähte sollen damit charakterisiert und Veränderungen der magnetischen Eigenschaften analysiert und diskutiert werden.

Der dritte Teil dieser Arbeit (Kapitel 5) befasst sich mit Untersuchungen des Wachstums und Zerfalls von Schichtsystemen auf den facettierten Substraten. Hierbei steht die Frage im Vordergrund, ob das Wachstumsverhalten von Schichtpaketen gezielt durch die Nanostrukturierung des Substrats verändert werden kann und ob damit die Einbringung einer periodischen Anordnung von Defektbereichen in die Schichtsysteme möglich ist.

In dieser Arbeit werden zunächst mit Eisen/Gold und Eisen/Silber rein metallische, kristalline Schichtsysteme ähnlicher struktureller Eigenschaften, aber unterschiedlicher Randlöslichkeit gewählt. Darüber hinaus bietet die Kombination Kohlenstoff/Kobalt, die sich durch sehr glatte Grenzflächen auszeichnet, ideale Bedingungen, um die Wachstumsveränderungen durch die Substratstruktur detaillierter aufzuzeigen und deren Auswirkungen im Verlauf des Vielfachschicht- 
aufbaus zu untersuchen. Neu zu konzipierende Messaufbauten zur gleichzeitigen in-situ Leitfähigkeitsmessung in verschiedenen Richtungen bezüglich der Substratstruktur sollen weitere Analysen des Wachstums- und Perkolationsverhaltens ermöglichen. Ergebnisse zur Leitfähigkeitsmessung während des Temperprozesses, sowie anschließende Textur- und Gefügeuntersuchungen sollen die Diskussion im Hinblick auf die Selbstordnung ergänzen.

Um ein vollständiges Bild vom strukturellen Aufbau der Schichtsysteme zu erzielen, sollen neben röntgenographischen Streumethoden (XRD), wie zum Beispiel Struktur- und Textumessungen, auch präparativ aufwändige Querschnittsuntersuchungen mittels Transmissionselektronenmikroskopie (TEM) erfolgen. Sie sollen das Wachstumsverhalten und die Mikrostruktur der Schichtsysteme und deren Veränderung durch die nanostrukturierte Unterlage offenlegen. Da durch das Wachstum auf den facettierten Substraten deutliche Intensitätsverluste bei der röntgendiffraktometrischen Charakterisierung der Schichtstrukturen auftreten, werden detaillierte Untersuchungen zum thermisch induzierten Zerfall der Schichtstruktur auf diesen Substraten mit Synchrotronstrahlung (am HASYLAB) durchgeführt.

Abschließend werden die Ergebnisse in Kapitel 6 zusammengefasst. 


\section{Experimentelle Methoden}

In diesem Kapitel werden zunächst die verwendete Herstellungsmethode und die Depositionsgeometrien erläutert. Es folgt eine Darstellung der im Rahmen dieser Arbeit verwendeten Charakterisierungsmethoden.

\subsection{Deposition der Proben durch Trioden-Magnetron-Sputtern im UHV}

Die Schichtabscheidung erfolgte im Ultrahochvakuum (UHV) bei einem Restgasdruck kleiner als $10^{-8}$ mbar. Der Basisdruck kann durch ein Kühlschild, das vor der Deposition mit flüssigem Stickstoff befüllt wird, weiter reduziert werden. Zur Deposition werden Trioden-Magnetron-Quellen eingesetzt, die in vier jeweils $90^{\circ}$ zueinander versetzten Positionen an der Kammer montiert werden können und $35^{\circ}$ zur Längsachse der Anlage geneigt sind. Der Abstand zwischen Quellen und Substraten auf dem Probenhalter im Zentrum der Kammer beträgt $23 \mathrm{~cm}$. Die Quellen werden mit Argon bei einem Basisdruck von ca. $1 \cdot 10^{-2}$ mbar betrieben. Dadurch ist die mittlere freie Weglänge auf wenige Zentimeter reduziert. Als Folge davon findet die Deposition auf den Substraten bei geringen kinetischen Energien $(0,5-10 \mathrm{eV})$ der auftreffenden Atome statt [Fre 87]. Daher ist das Herstellungsverfahren weniger stark gerichtet als andere, höherenergetische Verfahren, wie z.B. die Laserablation, was dort auch zur Beschichtung geometrisch abgeschatteter Bereiche führen kann. Die Aufwachsraten variieren materialabhängig von $0,3 \AA / \mathrm{s}$ bei Kohlenstoff ( $300 \mathrm{Watt}$ ) bis zu $5 \AA / \mathrm{s}$ bei Silber (200Watt) bei senkrechter Deposition. Sie werden während des Beschichtungsprozesses

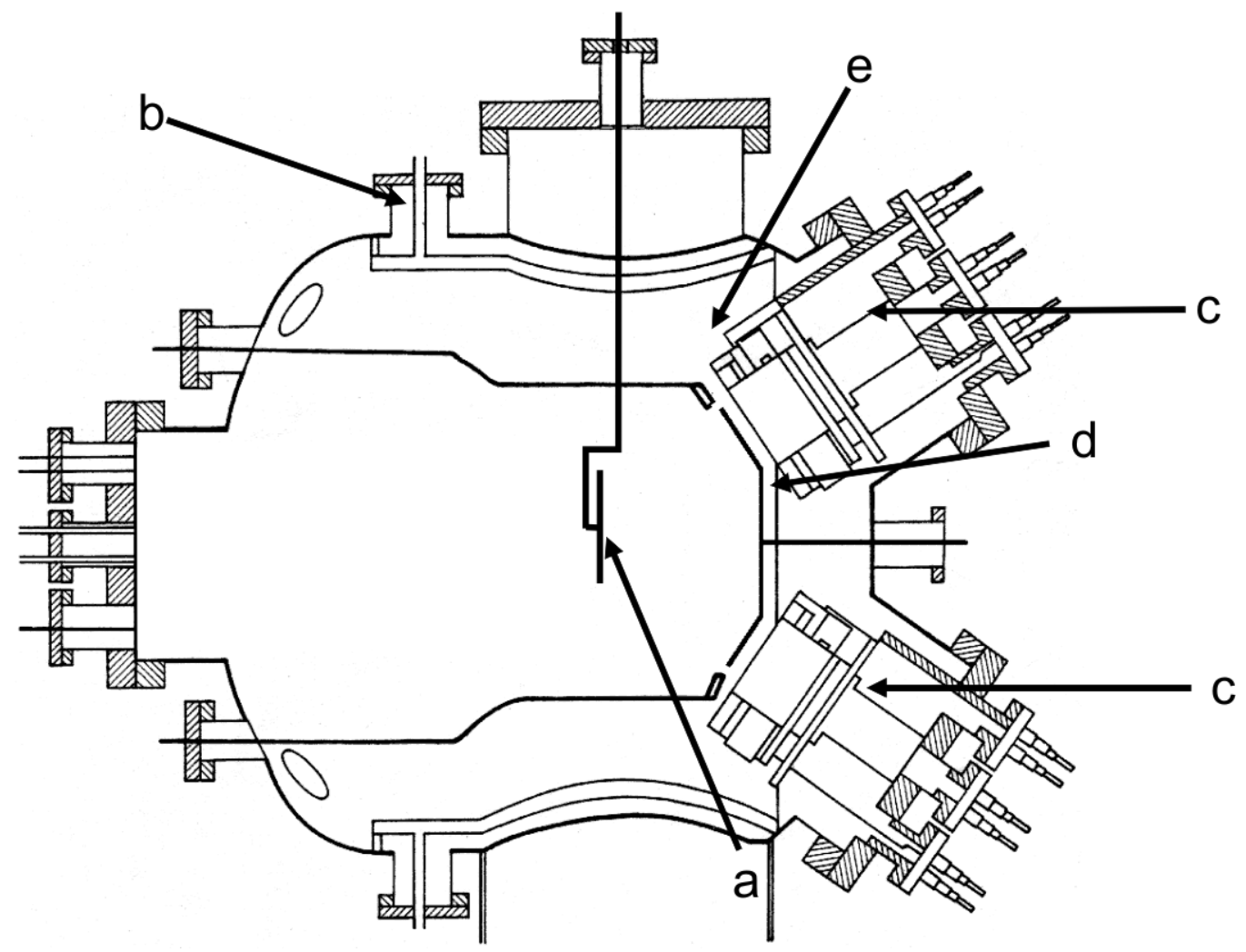

Abbildung 1: Querschnitt der UHV-Sputteranlage: a) Drehprobenhalter, b) Kühlschild,

c) Trioden-Magnetron-Sputterquellen, d) Quellendrehblende, e) Ratenmonitore. 
mittels Schwingquarz-Ratenmonitoren kontrolliert. Eine detaillierte Darstellung zu erzielbaren Aufwachsraten mit dem Depositionssystem findet sich in [Hof 91].

Durch eine Drehblende (Abbildung 1d) ist es möglich den Plasmaeinfluss der Quellen auf den Probenhalter während der Depositionspausen zu minimieren.

\section{Geometrie bei der Deposition von Vielfachschichten}

Es erfolgten Untersuchungen an Schichten, die auf glattem $(10 \overline{1} 0)-\mathrm{Al}_{2} \mathrm{O}_{3}$, sowie vollständig facettiertem $\mathrm{Al}_{2} \mathrm{O}_{3}$ hergestellt wurden (Eine genau Beschreibung dieser Oberflächenmorphologie erfolgt in Kapitel 3). Durch die spitzdachartige Oberfläche ist die Ausrichtung des Substrats bezüglich der Quellenanordnung bei der Abscheidung der Schichtsysteme wesentlich.

a)

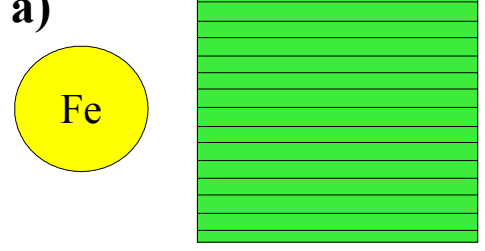

b)

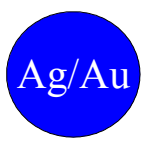

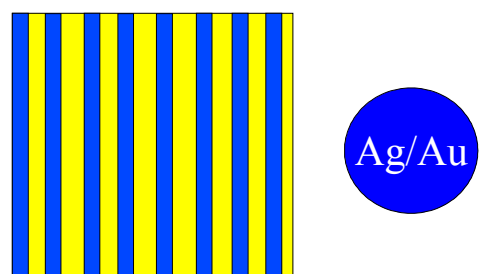

Abbildung 2: a) homogene (horizontale) b) präferentielle (vertikale) Deposition auf den unterschiedlichen Facettenseiten.

Es wurden sowohl Ausrichtungen der Facettenkämme in der Ebene der Quellen (Abbildung 2a), wodurch eine homogene Bedeckung beider Facettenseiten mit beiden Materialien erzielt wird, als auch senkrecht dazu (Abbildung 2b), was zu einer leicht präferentiellen Belegung der einzelnen Facettenseiten mit jeweils einem Beschichtungsmaterial führt, realisiert. Der Unterschied in der Schichtdicke durch den veränderten Depositionswinkel ist dabei kleiner als $8 \%$.

Für die Deposition der Nanodrähte konnte der Drehprobenhalter so weit zur Quelle gedreht werden, dass die Deposition unter sehr flachem Winkel von $3 \pm 1^{\circ}$ erfolgte.

\subsection{In-situ Vierpunktmessung in zwei Messrichtungen}

Während der Deposition wurden simultan 4-Punkt-Leitwertmessungen in zwei Richtungen durchgeführt, zum einen in Facettierungsrichtung, zum anderen senkrecht dazu. Zur Kontaktierung der Schicht wurden vorher Gold-Kontakte mittels Sputterns auf die Substrate aufgebracht. Eine Maske schränkte während der Deposition der Schichtpakete die beschichtete Fläche auf zwei senkrecht zueinander verlaufende Stege ein (siehe Abbildung 3).

Durch die Anordnung der Kontakte in Verbindung mit der Einschränkung des Depositionsbereichs entspricht die Geometrie weitgehend der van-der-Pauw-Methode [Pau 58], wobei ein gemeinsamer Goldkontakt für den Spannungs- und Stromkontakt senkrecht zum Messsteg unvermeidlich ist, um Unterschiede parallel und senkrecht zur Facettierungsrichtung nachweisen zu können.

Die Datenaufnahme erfolgte sequentiell, d.h. zunächst wurde ein Wert in paralleler Richtung, dann ein Wert in senkrechter Richtung ermittelt usw. Auf diese Weise kann eine Messwertbeeinflussung durch den Messstrom der jeweils anderen Richtung ausgeschlossen werden. Die Datenaufnahme erfolgte für die unterschiedlichen Messrichtungen jeweils über ein eigenständiges hochpräzises digitales Multimeter vom Typ HP 34401A. Der Messstrom kann im Bereich von 500nA bis 1mA gewählt werden. 
a)

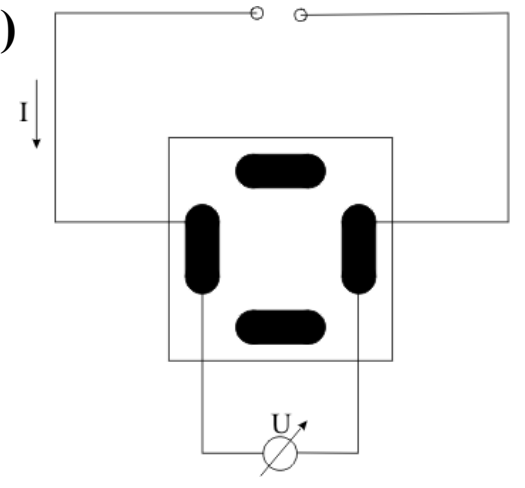

b)

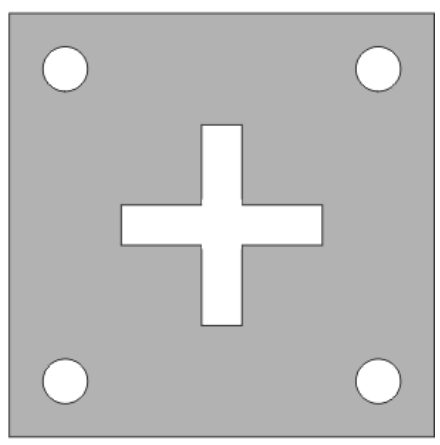

Abbildung 3: a) schematischer Aufbau der Leitfähigkeitsmessung (Kontaktierung exemplarisch für die horizontale Messrichtung) b) Depositionsmaske.

Die Multimeter wurden angesteuert und ausgelesen über eine mittels Labview ${ }^{\circledR}$ entwickelte Software. Diese erlaubte die Aufnahme von bis zu 5 Datenpunkten pro Sekunde in beiden Messrichtungen und eine Echtzeitdarstellung.

Durch die verwendete Steggeometrie ist es möglich, aus dem gemessenen Widerstand R unter Berücksichtigung der Schichtdicke $d$, den spezifischen Widerstand $\rho$ bzw. die Leitfähigkeit $\sigma \mathrm{zu}$ ermitteln.

$$
\rho=\frac{b \cdot d}{l} R=\sigma^{-1}
$$

Nur im Fall der Analyse der Schichtpaketen ist sowohl die Schichtdicke d als auch die Breite $b$ wohldefiniert. Die Deposition der Drähte unter flachem Winkel führt zu teilweise variierenden Drahtdicken und -breiten, weshalb bei der Diskussion der Drähte von der Angabe spezifischer Werte abgesehen wird. Um einen möglichst parallelen Verlauf der Äquipotentialflächen über den Messsteg zu erzielen, ist ein großes Aspektverhältnis von Breite $b$ zu Länge $l$ wünschenswert. Die Länge ist durch die Substratabmessungen auf $l=5,5 \mathrm{~mm}$ begrenzt, die Breite sollte $b=2 \mathrm{~mm}$ nicht unterschreiten, um Abschattungseffekte und daraus entstehende Schichtdickeninhomogenitäten zu vermeiden.

Durch die Geometrie der 4-Punkt-Messung erfolgt die Spannungsmessung praktisch stromlos (der Eingangswiderstand des Messgerätes beträgt $10 \mathrm{M} \Omega$ ), so dass an den Kontakten und in den Zuleitungen ein Spannungsabfall vermieden wird und nur der Widerstand der deponierten Schicht bestimmt werden kann.

Die Proben sind für die Messungen auf einem elektrisch isolierenden Keramikhalter (AluminiumSilikat, Festkeramik Nr. 9020, Fa. Gager GmbH, Dietzenbach) fixiert. Der gesamte Messaufbau ist auf einem Bornidrid-Heizer ( $>45 \mathrm{Watt} / \mathrm{cm}^{2}$ ) montiert, so dass direkt im Anschluss an die Deposition ohne Brechen des Vakuums Temperprozesse bis zu $600^{\circ} \mathrm{C}$ möglich sind.

\subsection{Röntgendiffraktometrie (XRD)}

Für die röntgendiffraktometrischen Messungen wurden zwei verschiedene Diffraktometer verwendet, ein 2-Kreis-Diffraktometer, sowie ein 4-Kreis-Diffraktometer. Zusätzlich erfolgten Messungen an einem Messplatz mit 2-Kreis-Goniometer am Synchrotronlabor HASYLAB, an der Beamline B2. 
Das 2-Kreis-Diffraktometer der Firma Siemens Typ D500 diente zur Struktur- und Phasenanalyse durch Untersuchungen in Bragg-Brentano-Geometrie. Eine umfassenden Beschreibung des Gerätes findet sich in [Kre 84]. Es wurden eine Kobaltröntgenröhre in Kombination mit einem Eisenfilter verwendet, wodurch die Analysen mit monochromatischer Strahlung der Wellenlänge $\lambda_{\mathrm{K} \alpha}=1,7903 \AA$ erfolgten. Apparativ ergibt sich eine Auflösungsbegrenzung von $0,005 \AA$ bei der Bestimmung von Netzebenenabständen. Der Probenraum des Diffraktometers kann bis zu einem Restgasdruck von $<3 \cdot 10^{-6} \mathrm{mbar}$ evakuiert werden. Durch einen heizbaren Probentisch ist damit die kontinuierliche Strukturanalyse während des Temperns bis zu $700^{\circ} \mathrm{C}$ möglich. Entsprechende Analysen im Rahmen dieser Arbeit erfolgten mit Heizraten von 0,2K/min.

Für Messungen am Hamburger Synchrotronstrahlungslabor HASYLAB wurde ein Messaufbau konstruiert, der vergleichbare in-situ Röntgenmessungen während eines Temperprozesses bis $600^{\circ} \mathrm{C}$ bei einem Restgasdruck von $<5 \cdot 10^{-6}$ mbar unter Ausnutzung der hohen Brillianz der Strahlquelle eines Speicherrings erlaubt. Die Messungen mit Heizraten von $1 \mathrm{~K} / \mathrm{min}$ erfolgten bei einer Wellenlänge der Strahlung von $\lambda=0,709494 \AA$, bestimmt mittels eines $\mathrm{LaB}_{6}-\mathrm{K}_{\mathrm{K}}$ istalls. Eine detaillierte Beschreibung des Messplatzes und des Goniometers findet sich in [Sht 03].

Die Texturanalysen erfolgten an einem 4-Kreis-Diffraktometer vom Typ X'Pert der Firma Phillips, ebenfalls mit Kobalt- $\mathrm{K}_{\alpha}$-Röntgenstrahlung. Mit einer Euler-Wiege kann die Probe unabhängig von der eingestellten Reflexbedingung in einer vollständigen Hemisphäre gedreht werden (Psi $=0-90^{\circ}$ und $\mathrm{Phi}=0-360^{\circ}$ ). Somit erhält man eine Polfigur, also eine von diesen beiden Winkeln abhängige Orientierungsverteilung der Kristallite, die die gewählte Reflexbedingung erfüllen. Weiterführende Grundlagen zur Texturanalyse und zum verwendeten Texturgoniometer finden sich in [Stu 00], [Dzi 00] und [Thi 04].

\section{Röntgenbeugung und atomare Streufaktoren}

Den grundlegenden Zusammenhang zwischen dem Beugungswinkel $\theta$ der Röntgenstrahlung und dem Netzebenenabstand d gibt die Bragg-Bedingung:

$$
2 \cdot d \cdot \sin \theta=n \cdot \lambda
$$

$\mathrm{n}$ ist hierbei die Ordnung des Beugungsmaximums.

Bei der Röntgenstrukturanalyse ist die gebeugte Intensität materialabhängig, da Röntgenstrahlung im Wesentlichen an der Elektronenhülle gestreut wird. Diese Abhängigkeit spiegelt sich im atomaren Streufaktor $\mathrm{f}(\mathrm{s})$ wider und ist im Wesentlichen proportional zur Zahl der Elektronen bzw. zur Ordnungszahl Z. Für Eisen, Silber und Gold ergeben sich dadurch Unterschiede im Streuverhalten im Winkelbereich der $(110)$ bzw. (111)-Kristallreflexe $\left(\sin \theta \cdot \lambda^{-1} \approx 0,2\right)$ von $\mathrm{f}(\mathrm{s})_{\mathrm{Fe}}=16,8$ $\mathrm{zu} f(\mathrm{~s})_{\mathrm{Ag}}=37,6 \mathrm{zu} \mathrm{f}(\mathrm{s})_{\mathrm{Au}}=65,8$. Weiterführende Erläuterungen zur Röntgenstreuung, sowie tabellierte Daten zu den atomaren Streufaktoren finden sich z.B. bei Warren [War 69].

\section{Reflexform und Mikrostruktur - Die Scherrer-Formel bei Schichtsystemen}

Die Reflexform ist stark von der Mikrostruktur der Probe bestimmt. Die Breite ist ein Maß für die typische Tiefe, aus der eine kohärente Überlagerung der Röntgenstrahlen erfolgen kann. Über die Scherrer-Formel [Cul 67] kann dazu eine charakteristische Kohärenztiefe ermittelt werden.

$$
D=\frac{0,9 \cdot \lambda}{B \cdot \cos \theta}
$$


$\mathrm{D}$ ist die Kohärenztiefe, $\lambda$ die Wellenlänge der verwendeten Röntgenstrahlung, $\mathrm{B}$ die Halbwertsbreite des Reflexes und $\theta$ dessen Winkelposition.

Oftmals wird D als Korngröße interpretiert. In geschichteten Systemen können aber innere Grenzflächen die Kohärenztiefe effektiv begrenzen. Daher wird im Weiteren der Wert, der sich aus der Scherrerformel ergibt, nicht als Korngröße sondern als Kohärenztiefe bezeichnet.

Faltung von Kristall- und Vielfachschichtsystemübergitter

Bei der Röntgenstrukturanalyse von Schichtsystemen beobachtet man in Bereichen von Winkeln, für die die Bragg-Bedingung durch die Ebenenabstände der untersuchten Kristallstruktur erfüllt ist, eine Modulation der Intensitätsverteilung durch eine periodische Abfolge von Satellitenreflexen. Diese beobachtete Überstruktur geht auf die Überlagerung der Rückstreuung der kristallinen Struktur (Kristallreflexe) und der künstlichen Übergitterstruktur durch die Schichtabfolge des Schichtsystems zurück. Für die Beugung an diesem Übergitter gilt wie für die Kristallbeugung die Bragg-Bedingung:

$$
2 \cdot \Lambda \cdot \sin \theta=n \cdot \lambda
$$

In diesem Fall entspricht $\Lambda$ der Periodizitätslänge der Übergitterstruktur. Die verwendete Wellenlänge des Röntgenstrahlenanteils der Synchrotronstrahlung betrug $\lambda=0,709494 \AA$. Geht man von einer Periodizitätslänge von $12 \mathrm{~nm}$ aus, ergibt sich für die 1. Ordnung der Beugung $(\mathrm{n}=1)$ ein Glanzwinkel von $2 \Theta=0,34^{\circ}$.

Aus dem Verhältnis der charakteristischen Ebenenabstände für das künstliche Übergitter $(\Lambda=12,0 \mathrm{~nm})$ und das Kristallgitter $\left(\mathrm{d}_{111}{ }^{\mathrm{Au}}=0,23546 \mathrm{~nm}\right)$ ergibt sich die Ordnung $n$ der Beugung am Vielfachschichtsystem, die im Bereich der Kristallreflexe Einfluss auf die Intensitätsverteilung hat.

$$
n=\frac{\Lambda}{d_{111}} \sim 50
$$

Die Faltung aus der kristallinen Struktur des Schichtmaterials und der künstlichen Überstruktur gibt die gemessene Intensitätsverteilung wieder. Einfache Simulationen dieser Verteilung sind auf Grundlage des Stufenmodels nach Locquet et al. möglich [Loc 89].

Im Bereich sehr kleiner Winkel knapp oberhalb der Totalreflexion ist die Intensitätsverteilung entsprechend nur durch die Beugung an der künstlichen Übergitterstruktur bestimmt.

\subsection{Rasterelektronenmikroskopie (SEM)}

Die Oberflächencharakterisierung mittels Rasterelektronenmikroskopie (SEM) erfolgte mit einem kommerziellen LEO SUPRA 35 Gemini FEG mit Feldemissions-Kathode.

Die Potentialdifferenz der Probe kann gegenüber der Beschleunigungsspannung bis auf $1 \mathrm{kV}$ reduziert werden, wodurch Aufladungseffekte bei der Analyse reduziert werden können. Auf eine zusätzliche Beschichtung der Proben mit Gold konnte daher verzichtet werden. Durch die Integration des Sekundärelektronen-Detektors in die Linse der Elektronenoptik wird eine Auflösung von bis zu $1,5 \mathrm{~nm}$ bei $20 \mathrm{kV}$ (bzw. $2,5 \mathrm{~nm}$ bei $1 \mathrm{kV}$ ) erzielt. 


\subsection{Rasterkraftmikroskopie (AFM)}

Die rasterkraftmikroskopischen Analysen wurden mit zwei unterschiedlichen kommerziellen Geräten durchgeführt. Die Analysen mit unbeschichteten Si-Spitzen erfolgten im kontaktfreien bzw. kontaktarmen Modus (TappingMode ${ }^{\circledR}$ ) mit einem MultiMode-AFM mit NanoScope-IIIa Controller (Fa. Digital Instruments, Santa Barbara, CA.). Zur Aufzeichnung der Phasenverschiebung verfügen die Geräte über ein Zusatzgerät, die Extender Box EX-2 (Digital Instruments). Die Messungen wurden bei Raumtemperatur und in Atmosphäre durchgeführt.

Typische Spezifikationen eines Si-Cantilevers sind:

- Spitzenradius $r=10 \mathrm{~nm}$

- Öffnungswinkel des Spitzenkonus: $\sim 10^{\circ}$

- Resonanzfrequenz: $270-390 \mathrm{kHz}$

- Federkonstante: $42-60 \mathrm{~N} / \mathrm{m}$

Im TappingMode wird der Cantilever zur Schwingung knapp unterhalb der Resonanzfrequenz angeregt. Es ist somit dynamisch möglich, geringe Wechselwirkungen der Spitze mit der Probenoberfläche festzustellen und über den Regelkreis mit dem Scanner die Probe entsprechend nachzuregeln.

Die im Rahmen dieser Arbeit dargestellten Rasterkraftmikroskopieanalysen unterscheiden sich bezüglich der dargestellten Information in Höhen-, Amplituden- und Phasenabbildung.

Die Höheninformation ist direkt durch die Auslenkung des z-Piezos zur Höhenregulierung der Probe zugänglich. Die Amplitudenabbildung gibt die Veränderung der Schwingungsamplitude durch die Wechselwirkung mit der Oberfläche wieder. Neben der Amplitudenveränderung kommt es aber vor allem zu einer Phasenverschiebung der Schwingung des Cantilevers gegenüber der Anregung. Diese Phasenverschiebung ist sensitiv für tribologische Eigenschaften, d.h. Veränderungen im Reibungs- bzw. Kohäsions- und Adhäsionsverhalten der untersuchten Oberfläche.

\section{Messfehler}

Neben Artefakten durch schlecht präparierte Spitzen, die zu Doppelabbildungen führen können oder Störungen durch schlechte Schwingungsdämpfung bzw. mangelhafte elektronische Entkopplung, treten auch Abbildungsfehler auf. Typische Abbildungsfehler an Kanten und die projektive Verbreiterung sphärischer Partikeldurchmesser wurden von verschiedenen Autoren diskutiert, die je nach Probenmorphologie unterschiedliche Modelle angenommen haben [Odi 94], [Kel 91], [Ram 98], [But 95].

Weitergehende Erläuterungen zur Rasterkraftmikroskopie sowie deren Abbildungsmoden und -fehler finden sich in einer Vielzahl Veröffentlichungen und Büchern. Eine Übersicht gibt der Artikel von Giessibl [Gie 03].

\section{Magnetische Rasterkraftmikroskopie (MFM)}

Die magnetischen Oberflächenanalysen mittels MFM wurden mit einem BioScope mit NanoScopeIIIa Controller (Fa. Digital Instruments, Santa Barbara, CA.) durchgeführt.

Verwendet wurden Whisker-Typ-Spitzen (Fa. NT-MDT) mit einer hartmagnetischen Kobalt- bzw. Kobaltsamariumbeschichtung. 
Spezifikationen:

- Spitzenradius $r<30 \mathrm{~nm}$

- Resonanzfrequenz: $115-190 \mathrm{kHz}$

- Federkonstante: $2-10 \mathrm{~N} / \mathrm{m}$

Die Abbildung der magnetischen Probeneigenschaften erfolgte durch den kombinierten TappingLiftMode. Dabei wird die Spitze in zwei Durchgängen über die Probe geführt. Im ersten erfolgt im oben erläuterten TappingMode eine Aufzeichnung der Topographie der Probenoberfläche, so dass im zweiten Durchgang die magnetische Spitze in einem konstanten Abstand (5nm-10nm) über die Probe geführt werden kann. Die Wechselwirkung der magnetischen Spitze mit der Streufeldverteilung der Probe führt zu Veränderungen in der Phase der Cantileverschwingung. Somit kann die Phasendarstellung im TappingLiftMode mit einer magnetischen Spitze die magnetische Domänenstruktur einer ferromagnetischen Probe abbilden.

Die Kraft zwischen Spitze und Probe kann im allgemeinen Fall beschrieben werden durch:

$$
F_{\text {mag }}=-\nabla \int_{\text {Spitze }} \boldsymbol{M}_{\text {Spitze }} \cdot \boldsymbol{H}_{\text {Probe }} d V
$$

Dabei ist $\boldsymbol{M}_{\text {Spitze }}$ die Magnetisierung der Spitze und $\boldsymbol{H}_{\text {Probe }}$ das Streufeld der Probe. Die verwendeten Whisker-Spitzen haben ein hohes Aspektverhältnis und sind idealerweise eindomänig mit einer Magnetisierungsrichtung entlang der Achse der Spitze. Die beschichtete Spitze kann in der Wechselwirkung mit dem Streufeld der Probe weder alleine durch die Wirkung des probennahen Pols (Monopolnäherung) noch durch die Kraft durch den Feldgradienten (Dipolnäherung) vollständig beschrieben sind. Daher werden in der häufig verwandten „PunktsondenApproximation“ effektive Monopol- $q_{\text {Spitze }}^{\text {effektiv }}$ bzw. Dipolmomente $\boldsymbol{m}_{\text {Spitze }}^{\text {effekiv }}$ zur Beschreibung der Spitzeneigenschaften verwendet [Har 94].

$$
F_{\text {mag }}=\left(q_{\text {Spitze }}^{\text {effekiv }}-\boldsymbol{m}_{\text {Spitze }}^{\text {effekiv }} \nabla\right) \cdot \boldsymbol{H}_{\text {Probe }}
$$

Dabei führt gerade die Berücksichtigung von Monopol- und Dipolmoment zu der Tatsache, dass beim MFM im dynamischen Modus nicht allein die zweite Ableitung der Streufeldkomponenten detektiert wird, sondern vielmehr auch Beiträge der ersten Ableitungen und Beiträge des Streufelds selbst. Die tatsächliche Streufeldverteilung der MFM-Spitze kann experimentell durch ElektronenHolographie analysiert werden [Mat 93].

\subsection{Transmissionselektronenmikroskopie (TEM)}

Die Untersuchung der Mikrostruktur der Schichtsysteme im Querschnitt wurde mittels konventioneller Transmissionselektronenmikroskopie (TEM) an einem Philips 420ST EM (Beschleunigungsspannung: 120keV) durchgeführt. Die hochauflösenden Transmissionselektronenmikroskopie- (HRTEM) und die Raster-Transmissionselektronenmikroskopie- (STEM) Analysen erfolgten an einem Philips CM200 FEG1 (IV. Physikalisches Institut der Universität Göttingen) mit einer Beschleunigungsspannung von $200 \mathrm{keV}$.

Querschnittspräparation

Damit die Proben für die Analysen im TEM durchstrahlbar sind, müssen diese auf eine Schichtdicke $<60 \mathrm{~nm}$ gedünnt werden. Dazu wird üblicherweise eine Kombination aus einem mechanischen bzw. 
chemischen Abtragsverfahren, sowie dem Ätzen mittels Ionenstrahl benutzt. Um einen entsprechenden Querschnitt einer Probe zu ermöglichen, wird diese in einen Edelstahlzylinder mittels Epoxy-Kleber (G2, Fa. Gatan) eingebettet. Dieser wird im Folgenden senkrecht zur Zylinderachse in Scheiben einer Dicke von $0,8 \mathrm{~mm}$ gesägt.

Mit Schleifpapier sukzessiv steigender Körnung (800-2400) und einem Handschleifgerät (Disk Grinder, Fa. Gatan) wird die Dicke dieser Scheiben bis auf $50 \mu \mathrm{m}$ reduziert. Im zentralen Bereich der Scheiben, in dem der Querschnitt des Schichtsystems eingebettet ist, wird die Schichtdicke lokal mit einem Muldenschleifgerät (Dimple Grinder, Fa. Gatan) weiter bis auf $<20 \mu \mathrm{m}$ reduziert. Im letzten Schritt erfolgt der Abtrag durch Ionenbeschuss (Dual Ion Mill, Modell 600, Fa. Gatan) mittels Argon-Ionen (1-5kV, 0,5mA) unter einem Winkel von 11-15', bis ein möglichst kleines Loch in der Probe entstanden ist. Am Lochrand entstehen somit Probenbereiche, in denen die durchstrahlbare Schichtdicke erreicht und eine mikrostrukturelle Analyse somit möglich ist. Eine ausführlichere Darstellung der Querschnittspräparation findet man in [Kau 03].

Eine besondere Herausforderung stellen die stark unterschiedlichen Härten von Substrat und metallischem Schichtsystem dar, welche beim Ionenätzen zu einem präferentiellen Abtrag der Schicht führen. Daher muss der Ionenbeschuss auf einen Sektor beschränkt werden, in dem das Schichtsystem durch das Substrat geschützt wird, um einen vollständigen Abtrag der zu untersuchenden Schicht vor Erreichen einer hinreichend geringen Schichtdicke zu vermeiden. Eine umfassende Darstellung des Sektorätzens gibt Hartung [Har 96].

\subsection{Profilometrie}

Zur Bestimmung der Schichtdicke deponierter Filme wurde neben dem AFM und TEMQuerschnittspräparationen auch ein Profilometer (Fa. Feinprüf Perthen) verwandt. Das mechanische Abtastsystem führt dabei eine Nadel mit einer Auflagekraft von $<0,8 \mathrm{mN}$ über die Probenoberfläche. Vertikale Auslenkungen werden induktiv gemessen, so dass Schichtdicken ab ca. $30 \mathrm{~nm}$ an Kanten (durch Abdeckung bei der Deposition) mit einer Genauigkeit von 5nm messbar sind.

\subsection{Das Feldionenmikroskop (FIM) und die tomographische Atomsonde (TAP)}

Die tomographische Atomsonde (TAP), welche vereinfacht als eine Kombination eines Feldionenmikroskops (FIM) und eines positionssensitiven Detektors (PSD) gesehen werden kann, ermöglicht bei atomarer Auflösung eine dreidimensionale Untersuchung eines Analysevolumens. Eine detaillierte Darstellung des Feldionenmikroskops und der Atomsonde ist bei [Mil 96] zu finden.

Als Probengrundlage dienen elektrolytisch gedünnte Wolframspitzen mit einem Krümmungsradius von 10 bis $50 \mathrm{~nm}$, auf die mittels Sputtern die zu analysierenden Materialien aufgebracht werden. Die starke Krümmung der Spitzen muss bei der Übertragung der Ergebnisse auf planare Schichtsysteme berücksichtigt werden. Das Gefüge (und die Orientierung von Wachstumssäulen) kann dadurch verändert sein. Die Analyse erfolgt im Hochvakuum $\left(10^{-7}-10^{-8} \mathrm{~Pa}\right)$. In einem Abstand von $\mathrm{R}=50 \mathrm{~mm}$ befinden sich im FIM-Modus zwei Kanalplatten zur Signalverstärkung und ein Bildschirm. Zur Abbildung wird in der Hauptkammer durch ein Bildgas (z.B. He oder Ne) ein Druck von $5 \cdot 10^{-3} \mathrm{~Pa}$ eingestellt. Durch die Vorspannung von $\mathrm{U}=20 \mathrm{kV}$ der Spitze gegenüber dem 
Bildschirm werden die Bildgasatome polarisiert und zur Spitze hingezogen. Das elektrische Feld $E$ (bis zur 50V/nm) der Spitze ist:

$$
E=\frac{U}{k_{f} \cdot r}
$$

$k_{f}$ ist ein Geometriefaktor (5-7), der von der Spitzenform abhängig ist, $r$ ist der mittlere Krümmungsradius der Spitze. An der Spitze kommt es zur Ionisation der Bildgasatome an den Kanten, so dass diese radial von der Spitze weg in Richtung der Kanalplatten beschleunigt werden. Auf den Kanalplatten werden Elektronenlawinen ausgelöst, die zu Leuchtpunkten auf dem Bildschirm führen.

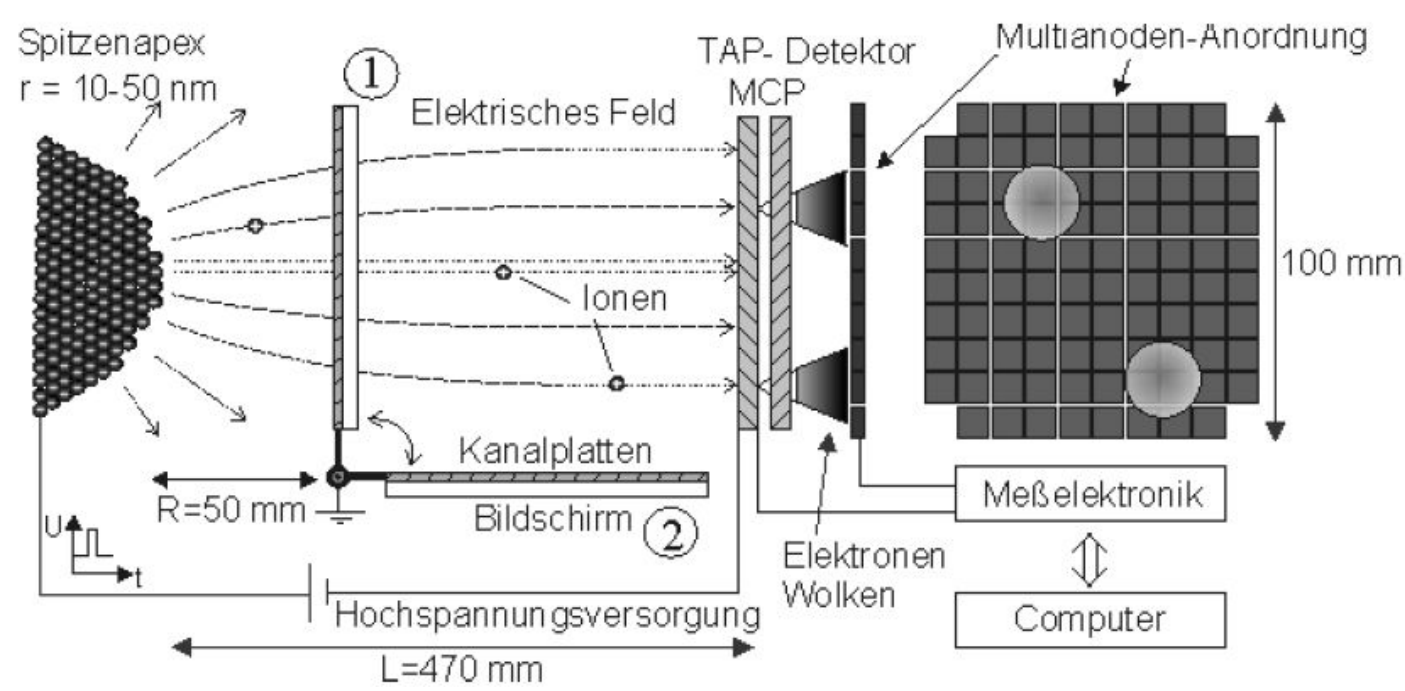

Abbildung 4: Aufbau eines Feldionenmikroskops mit dreidimensionalem Detektor; (1) FIM-Modus (2) 3D-Atomsonden-Modus. Abbildung aus [Klu 03].

Um das Gerät für die dreidimensionale, tomographische Analyse zu nutzen, muss das Bildgas entfernt und der Bildschirm weggeklappt werden. Über einen zusätzlichen Spannungspuls von 15$20 \%$ der Grundspannung kommt es zu einer Feldverdampfung am Apex der Spitze. Über eine Flugzeitmassenspektrometrie ist eine chemische Identifizierung der Ionen möglich. Der PSD ermöglicht die Bestimmung der lateralen Position der verdampften Ionen unter der Annahme einer Punktprojektion. Dabei erfolgt die Positionierung aus den Ladungsschwerpunkten der Elektronenwolken auf der Multianode. Die Positionsbestimmung in Richtung der Achse der Probenspitze erfolgt aus der Anzahl der feldverdampften Atomlagen. Mit diesen Informationen ist es möglich, eine dreidimensionale Rekonstruktion des analysierten Probenvolumens mit chemischer Identifizierung der Atome zu erzielen. Für die Darstellung wird eine Effizienz, im Wesentlichen beschränkt durch den PSD, von ca. 50\% der feldverdampften Atome erreicht. Weitere Erläuterungen zum Verfahren und zur Bildrekonstruktion sind in [Lan 02] und [Klu 03] zu finden.

\subsection{Magnetisierungsverhalten mittels magneto-optischen Kerr-Effekts (MOKE)}

Zur Charakterisierung des Magnetisierungsverhaltens der Nanodrähte erfolgten Untersuchungen mittels Magneto-Optischen Kerr-Effekts (MOKE) an den präparierten Proben. Der Kerr-Effekt kann in verschiedenen Geometrien genutzt werden: Beim polaren Kerr-Effekt ist die 
Magnetisierung senkrecht zur Filmebene. Wenn die Magnetisierung in der Ebene liegt, kann man den longitudinalen oder transversalen Kerr-Effekt messen. Beim longitudinalen Kerr-Effekt liegt die Magnetisierung in der Ebene, die durch ein- und ausfallenden Lichtstrahl gegeben ist, im Falle des transversalen Kerr-Effektes liegt sie senkrecht dazu (Abbildung 5). Der im Rahmen dieser Arbeit genutzte Aufbau gestattet die Messung des longitudinalen Kerr-Effektes.
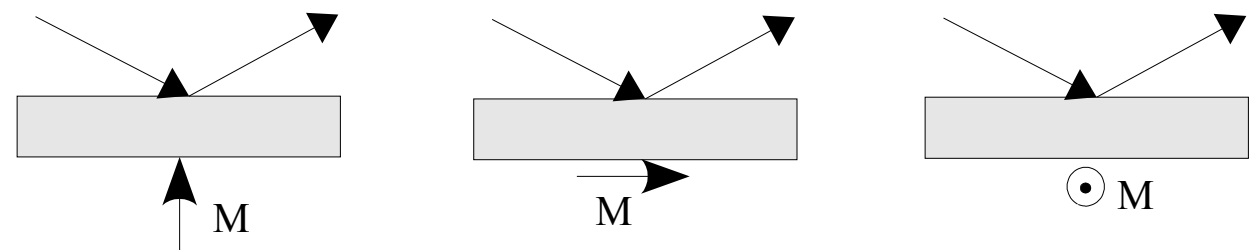

Abbildung 5: Polarer, longitudinaler und transversaler Kerr-Effekt.

Abbildung 6 zeigt eine schematische Darstellung des genutzten Aufbaus (1. Physikalisches Institut, Universität Göttingen), der dem eines PCSA-Ellipsometers (Polarisator-Compensator-SampleAnalysator) [Azz 77] entspricht.

Laserlicht der Wellenlänge $633 \mathrm{~nm}(2 \mathrm{~mW})$ wird durch einen Polarisator, einen festen Kompensator und einen zweiten, mit $50 \mathrm{kHz}$ modulierten Kompensator, auf die in eine Anordnung von Helmholtzspulen befindliche Probe gelenkt. Von dort trifft das Licht durch den Analysator auf einen

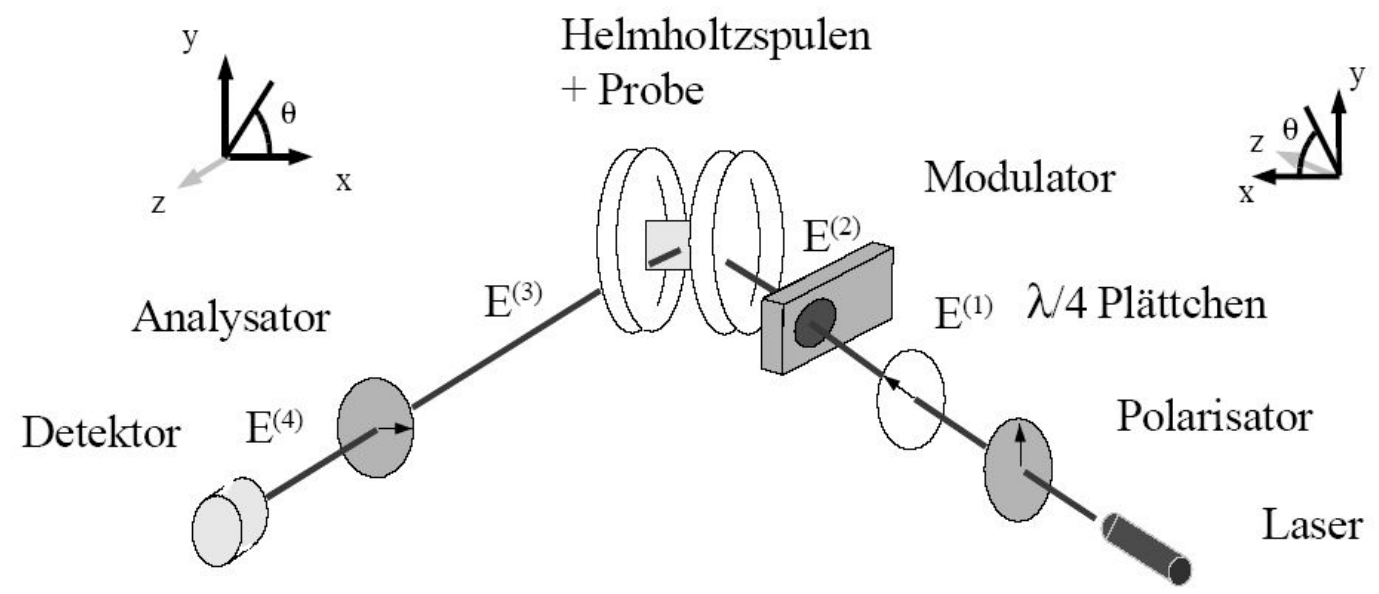

Abbildung 6: Aufbau des genutzten MOKE-Messstands mit PCSA-Modulationstechnik. Abbildung aus [Mün 97].

Photowiderstand als Detektor. Die gemessene Intensitätsmodulation wird mit der Modulationsfrequenz des Modulators verglichen und mit einem Lock-In-Verstärker quantitativ bestimmt. Durch eine Variation des Magnetfeldes in dem Spulenpaar lässt sich auf diese Weise ein direkt zur Magnetisierung der Probe proportionales Signal registrieren. Der Aufbau erlaubte Messungen bis zu einem Feld von 850 Oe bei einer Empfindlichkeit von $0,04 \cdot 10^{-3} \% / \mathrm{mV}$. Die typische Signalstärke beträgt $1 \mathrm{mV}$ bei einem Messfehler von $0,005 \mathrm{mV}$. Eine detaillierte Darstellung des Aufbaus und des MOKE findet sich in [Mün 97]. Messungen an Kobaltdrähten und -partikelanordnungen erfolgten an einem vergleichbaren Aufbau (PTB Braunschweig), der Felder von bis zu 3000Oe gestattet. 


\section{Thermische induzierte Veränderungen von m-plane Saphir}

Die richtige Auswahl und Präparation der Substrate ist ein zentraler Grundpfeiler der vorliegenden Arbeit. Wesentlich ist, dass die Substrate bei den anschließenden Wärmebehandlungen der abgeschiedenen Schichten weitgehend unverändert bleiben. Außerdem ist ein inertes Substrat besonders wünschenswert, damit Grenzflächenreaktionen und Phasenbildung bei der Deposition und späterer Temperung keinen Einfluss auf die resultierende Mikrostruktur haben. Elektrisch isolierendes Verhalten ist für eine Charakterisierung aufgebrachter Strukturen durch Leitwertmessungen unabdingbar.

Präparativ sind sehr hohe Anforderungen an die Periodizität der Substratoberfläche gestellt. Genaue Kenntnisse und Optimierung der Herstellungsbedingungen sowie eine sehr gute Reproduzierbarkeit sind notwendig. Vergleichende Versuche auch mit Laserstrukturierung oder Ionenstrahlbehandlung solche Oberflächenmodifikationen entsprechender Größen zu erreichen und dabei geeignete Unterlagen für die weiteren Untersuchungen herzustellen, ergaben, dass mittels der selbstorganisierten Facettierung der (10 10$)$-Saphiroberfläche die besten Ergebnisse erzielt werden konnten. $\mathrm{Al}_{2} \mathrm{O}_{3}$ ist chemisch inert und als Isolator mit einer Bandlücke von $\sim 9 \mathrm{eV}$ für Transportmessungen sehr gut geeignet.

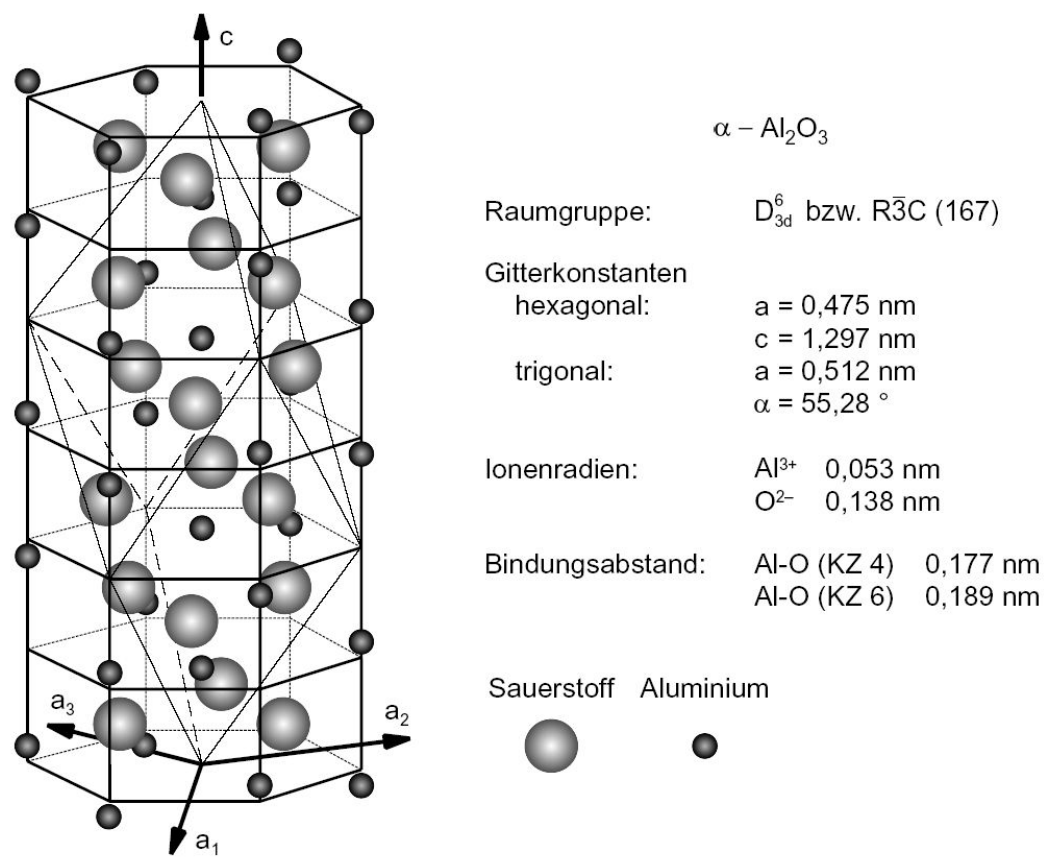

Abbildung 7: Hexagonale und trigonale Einheitszelle von $\alpha-\mathrm{Al}_{2} \mathrm{O}_{3}$ nach [Kro 57] und [Fra 99].
Bei dieser gezielten Ausnutzung selbstorganisierter Bildung regelmäßiger Oberflächenmorphologien ist eine Kenntnis der treibenden Kräfte wünschenswert, erreichbar durch Analyse der einzelnen Stadien der Facettierung. Die Facettenentstehung, das Wachstum der Facetten, ein Zusammenwachsen verschiedener Bereiche, in denen die Facettierung begonnen hat und der damit einhergehende Vergröberungsprozess, konnten beobachtet werden. Die Güte der Facettierung zeigt eine klare Temperaturabhängigkeit. Schließlich werden die Ergebnisse eingeordnet in den energetischen Hintergrund der Facettierung niedrig indizierter Kristallflächen. 


\subsection{Struktur und Indizierung von $\mathrm{Al}_{2} \mathrm{O}_{3}$}

$\alpha-\mathrm{Al}_{2} \mathrm{O}_{3}$ ist im System Al-O die einzige thermodynamisch stabile Phase. Daneben sind ein Reihe weiterer, metastabiler $\mathrm{Al}_{2} \mathrm{O}_{3}$-Formen bekannt. Hierzu zählt die bekannteste Modifikation $\gamma-\mathrm{Al}_{2} \mathrm{O}_{3}$, das durch ein kubisches, tetragonal verzerrtes Spinellgitter gekennzeichnet ist. Allen Modifikationen des $\mathrm{Al}_{2} \mathrm{O}_{3}$ ist gemeinsam, dass sie beim Erhitzen, teils über Zwischenstufen, bei $1200^{\circ} \mathrm{C}$ in $\alpha-\mathrm{Al}_{2} \mathrm{O}_{3}$ übergehen. Eine Übersicht, Phasendiagramm und Diskussion zu den gefundenen Modifikationen findet sich bei [Lan 91].

$\alpha-\mathrm{Al}_{2} \mathrm{O}_{3}$ hat eine hexagonal-rhomboedrische Kristallstruktur (die Kristallklasse gehört ins trigonale Kristallsystem) (Abbildung 7). Die trigonale Einheitszelle enthält 4 Aluminium- und 6 Sauerstoffatome. Betrachtet man die geometrisch anschaulichere hexagonale Zelle, so enthält diese 12 Al- und 16 O-Atome. Die Sauerstoffionen bilden in der hexagonal-dichtesten Packung Ebenen der Stapelfolge ABAB- in (0001)-Richtung aus. Die Oktaederplätze diese Gitters werden nur zu 2/3 mit den Aluminiumkationen besetzt, was eine Tetraederverzerrung des Kristallgitters zur Folge hat. Außerdem unterscheidet man drei unterschiedliche Kationenebenen (in (0001)-Richtung) a, b und c. Die komplette Stapelfolge ergibt sich zu A-a-B-b-A-c-B-a-A-b-B-c-A [Kro 57].

Gemäß der vierzähligen, hexagonalen Indizierung ergeben sich die in Tabelle 1 bezeichneten niedrig indizierten Ebenen des $\mathrm{Al}_{2} \mathrm{O}_{3}$.

\begin{tabular}{|c|c|}
\hline Notation & $\begin{array}{l}\text { Miller-Bravais } \\
\text { Indizierung }\end{array}$ \\
\hline Basal (c-plane) & $(0001)$ \\
\hline rhomboedrisch (R-plane) & $(\overline{1} 012)$ \\
\hline erste Ordnung prismatisch (M-plane) & $(\overline{1} 010)$ \\
\hline zweite Ordnung prismatisch (A-plane) & $(1 \overline{2} 10)$ \\
\hline
\end{tabular}

Tabelle 1: Notation und Indizierung niedrig indizierter Ebenen in $\alpha-\mathrm{Al}_{2} \mathrm{O}_{3}$.

Abbildungen 8 und 9 zeigen die Ionenanordnung auf diesen Kristallflächen im unrelaxierten Zustand, d.h. eine Anordnung, wie sie dem im Volumen entspricht. An einer Oberfläche kommt es $\mathrm{zu}$ Verschiebungen aus diesen Atompositionen, da die Bindungspartner fehlen und sich somit eine andere Position als Gleichgewichtsposition einstellt (Rekonstruktionen) [Har 80]. 


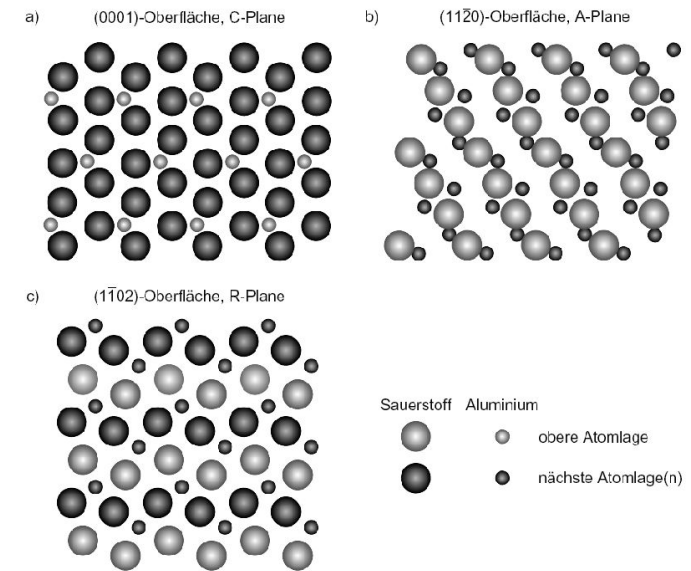

Abbildung 8: Struktur verschiedener Oberflächen von $\alpha-\mathrm{Al}_{2} \mathrm{O}_{3}$ nach [Fra 99].

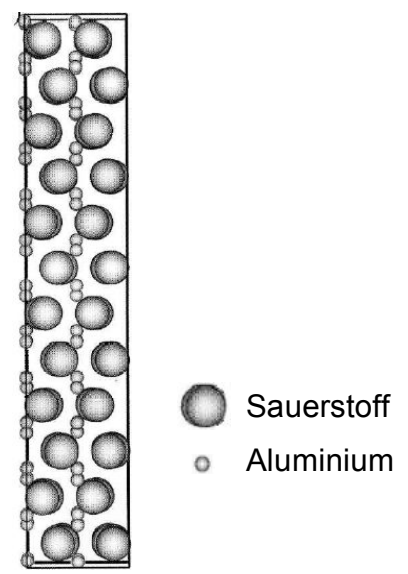

Abbildung 9: m-plane Oberfläche von $\mathrm{Al}_{2} \mathrm{O}_{3}$ nach [Heu 98].

Für $\mathrm{Al}_{2} \mathrm{O}_{3}$ werden nach Temperbehandlungen solche Oberflächenrekonstruktionen bei einzelnen Seiten beobachtet, z.B. die $\mathrm{Al}_{2} \mathrm{O}_{3}-(11 \overline{2} 0)-(12 \times 4)$ von Schildbach et al. [Sch 92] . Für die Analysen dieser Arbeit ist aber die Betrachtung der nicht relaxierten Atomposition in Abbildungen 8 und 9 durchweg ausreichend.

Die im Folgenden dargestellten Ergebnisse zur Facettierung von Saphir beziehen sich ausschließlich auf Substrate mit einer Ausgangsorientierung entsprechend einer (10 10 10)-Oberfläche mit einer spezifizierten Genauigkeit der Orientierung von 0,3 . Typische Substratgrößen sind $10 \mathrm{~mm} * 10 \mathrm{~mm} * 0,5 \mathrm{~mm}$, eine weitere Spezifikation zur Oberflächenbeschaffenheit durch den Hersteller (CrysTec, Berlin) ist RMS $=0,12 \mathrm{~nm}$ für die mittlere Rauigkeit im typischen Probenbereich von $1 \mu \mathrm{m}^{2}$. Um eine Facettierung der Oberfläche zu erzielen, ist eine Temperung der Substrate an Luft erforderlich. Die Temperungen bis zu $1600^{\circ} \mathrm{C}$ erfolgten in einem Hochtemperaturofen, wobei die Substrate in einem teilweise bedeckten $\mathrm{Al}_{2} \mathrm{O}_{3}$-Tiegel in der temperaturhomogenen Ofenmitte positioniert wurden. Die für die Dünnschichtabscheidung übliche Reinigung der Substrate mit Flusssäure, Trichlorethen, Aceton und Methanol zeigte keinen Einfluss auf die Facettierungsmorphologie.

\subsection{Frühstadien der Facettierung}

Im Vergleich zu Metallen [Ozc 94] und Halbleitern [Pha 91] ist die Dauer bis zur Nukleation von Facetten bei Keramiken lang. Während bei Metallen und Halbleitern schon nach wenigen Sekunden eine Facettierung beobachtet werden kann, sind bei $\alpha-\mathrm{Al}_{2} \mathrm{O}_{3}$ Stunden notwendig. Bei diesen Untersuchungen ist die Temperungstemperatur $\mathrm{T}$, die zur Facettierung der Oberfläche führt im Verhältnis zur Schmelztemperatur $\mathrm{T}_{\mathrm{m}}$ für Saphir am höchsten, was die Diffusion und somit eine schnelle Facettierung des Saphirs beschleunigen sollte $\left(T / \mathrm{T}_{\mathrm{m}}(\mathrm{Si})=0,60, \quad \mathrm{~T} / \mathrm{T}_{\mathrm{m}}(\mathrm{Ag})=0,34\right.$ und $\left.\mathrm{T} / \mathrm{T}_{\mathrm{m}}\left(\mathrm{Al}_{2} \mathrm{O}_{3}\right)=0,68\right)$. Dahingegen ist der Aufbau als Ionenkristall für den Materialtransport hinderlich.

Temperatur und Dauer der Temperung haben wesentlichen Einfluss auf die resultierende Morphologie und auf die Perfektion der Facettierung. Da beide genannten Parameter nicht 
unabhängig voneinander sind, wurden die Stadien der Facettierung nur durch Variation der Temperatur bei gleichbleibender Temperdauer von 24 Stunden untersucht.

Die vergleichsweise lange Temperdauer wurde in Hinblick auf kinetische Barrieren für die Ausbildung und Bewegung von speziell orientierten Facettenseiten gewählt. Eine verbesserte periodische Anordnung wird erreicht, wenn auch langsame Prozesse, die mit langreichweitigen Wechselwirkungspotentialen verknüpft sind, zur Ordnungseinstellung beitragen.

Unterhalb von $1300^{\circ} \mathrm{C}$ ist auch nach einer Temperung für $24 \mathrm{~h}$ keine Veränderung der Oberflächenbeschaffenheit hin zu einer geordneten Struktur erkennbar.
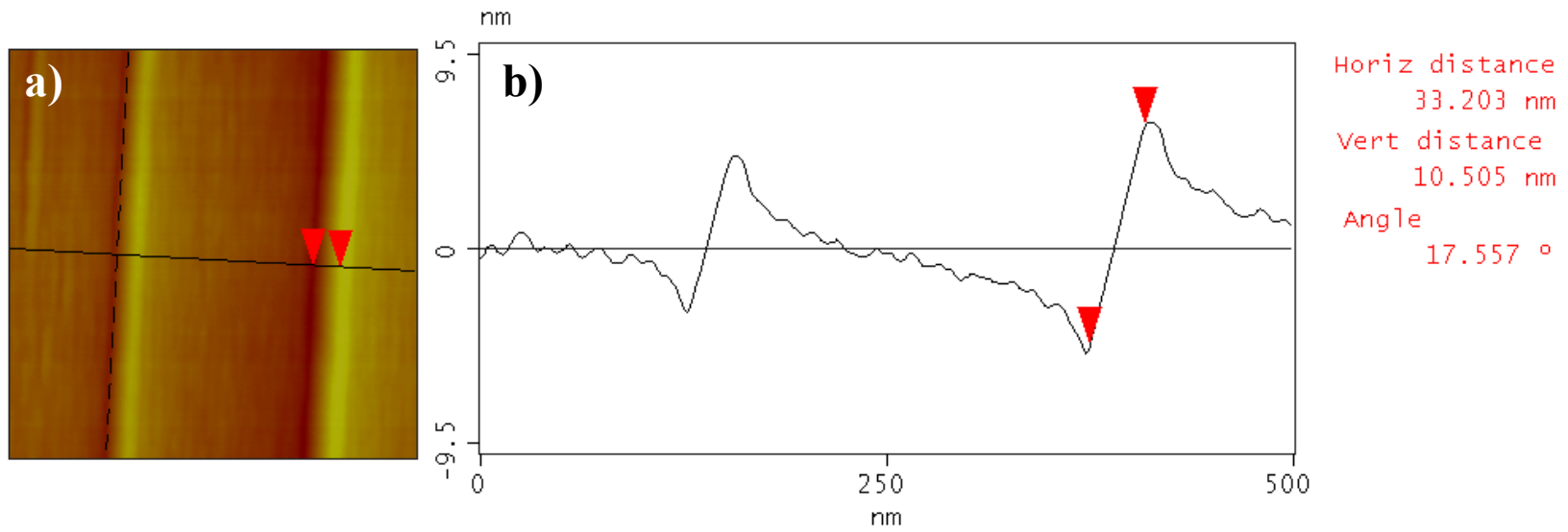

Abbildung 10: AFM-Daten eines 500nm² Ausschnitts; Temperung: $24 \mathrm{~h}$ bei $1300^{\circ} \mathrm{C}$. a) Höhendaten und b) Schnitt entlang der im Bild eingezeichneten Gerade. Rote Dreiecke markieren in Schnitt- und Bilddarstellung gleiche Orte.

Die Ergebnisse zur Temperung bei $1300^{\circ} \mathrm{C}$ erlauben, den Beginn, also die Frühstadien der Facettierung zu untersuchen. Abbildung 10 zeigt AFM-Daten: Die Höheninformation (a) und eine Schnittdarstellung (b) eines $500 \mathrm{~nm}^{2}$ Ausschnitts eines $24 \mathrm{~h}$ bei $1300^{\circ} \mathrm{C}$ getemperten Substrats. Es handelt sich um eine stark überhöhte Darstellung. Der Bildbereich zeigt zwei parallele Linien maximaler Höhe, die ca. 250nm separiert sind. Der Verlauf dieser Facettierungslinien erfolgt parallel zu den Kanten des Substrats und wird als Facettierungsrichtung (Abbildung 11) bezeichnet. Jeweils ca. 33nm links der Maxima finden sich vergleichbare Linien minimaler Höhe, die vertikale Distanz beträgt ca. 10nm. Diese Flächen zwischen der Facettenlinie minimaler Höhe (Facettental) und maximaler Höhe (Facettenkamm oder -spitze) bilden die Facettenseiten.

Die Facettierung des Substrats ist noch unvollständig. Die Facettenseiten unterscheiden sich sehr stark. Während die in der Schnittdarstellung linke Facettenseite sehr glatt ist und einen Winkel von

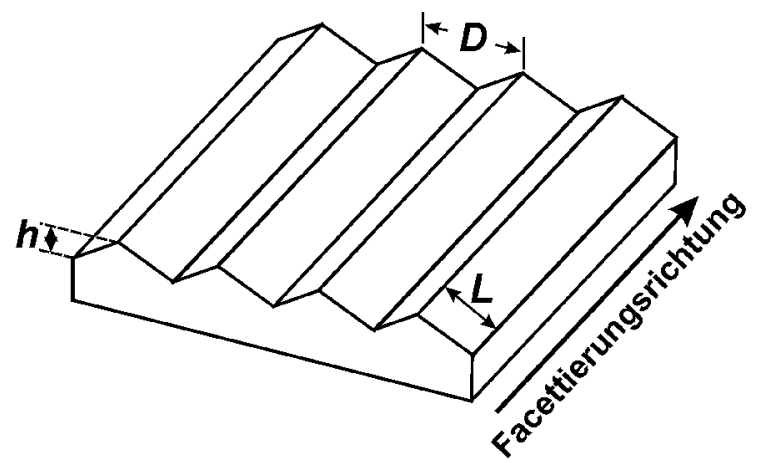

Abbildung 11: Definition der Facettierungsrichtung, Facettierungsperiode D, Facettenseitenbreite L und Facettenhöhe h. ca. $17,6^{\circ}$ aufweist, ist die zweite Seite durch eine sehr raue Oberfläche gekennzeichnet. Im Bereich des Kamms und des Tals finden sich besonders steile Abschnitte, die 15-20nm breit sind. Wie ein Vergleich mit der dreidimensionalen Darstellung in Abbildung 12 (die Probe war gegenüber Abbildung 10a um $180^{\circ}$ gedreht eingebaut) zeigt, handelt es sich bei der Oberflächenmorphologie zwischen diesen Anfangsbereichen nicht um eine unkorrelierte Rauigkeit, vielmehr ist gut $\mathrm{zu}$ erkennen, dass es sich auch auf der flachen Seite um eine quasiperiodische Morphologie von kleineren Facetten (im Weiteren auch als Nanofacetten bezeichnet) handelt. 


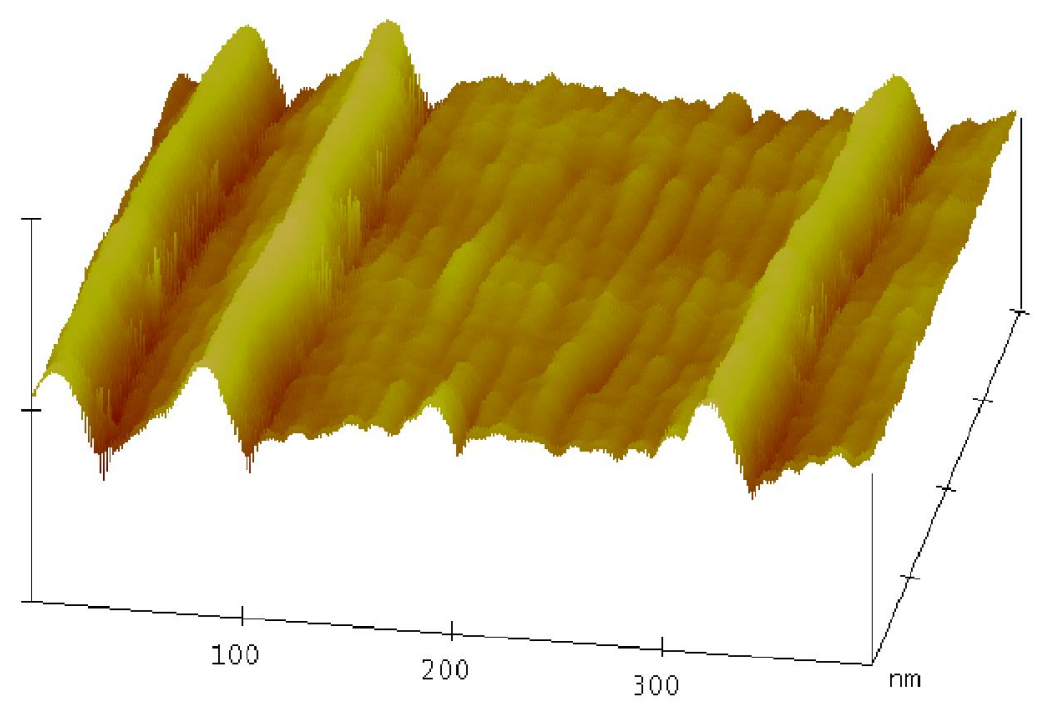

Abbildung 12: AFM-Daten: dreidimensionale Darstellung der

Höhendaten; Temperung: $24 \mathrm{~h}$ bei $1300^{\circ} \mathrm{C}$; z-Skaleneinheit: $10 \mathrm{~nm}$; sehr glatte Facettenseiten rechts und korrelierte Rauigkeit auf der „Ausgleichsseite“ links.

Die Facettierung der $(10 \overline{1} 0)-\mathrm{Al}_{2} \mathrm{O}_{3}$-Kristallfäche führt insbesondere in den Anfangsstadien zu sehr ungleichen Facettenseiten. Wie sich diese sehr unterschiedliche Perfektion der Facettenseiten bei einer vollständig facettierten Oberfläche auswirkt und was die physikalische Ursache für die korrelierte Rauigkeit auf der flachen Facettenseite ist, konnte durch TEM-Querschnittsuntersuchungen an 24 Stunden bei $1550^{\circ} \mathrm{C}$ getemperten Substraten geklärt werden. Darüberhinaus ermöglicht diese Methode im Gegensatz zur Rasterkraftmikroskopie Orientierungsbestimmungen, wodurch Facettierungsrichtung und Facettenseiten kristallographischen Ebenen zugeordnet werden können. 

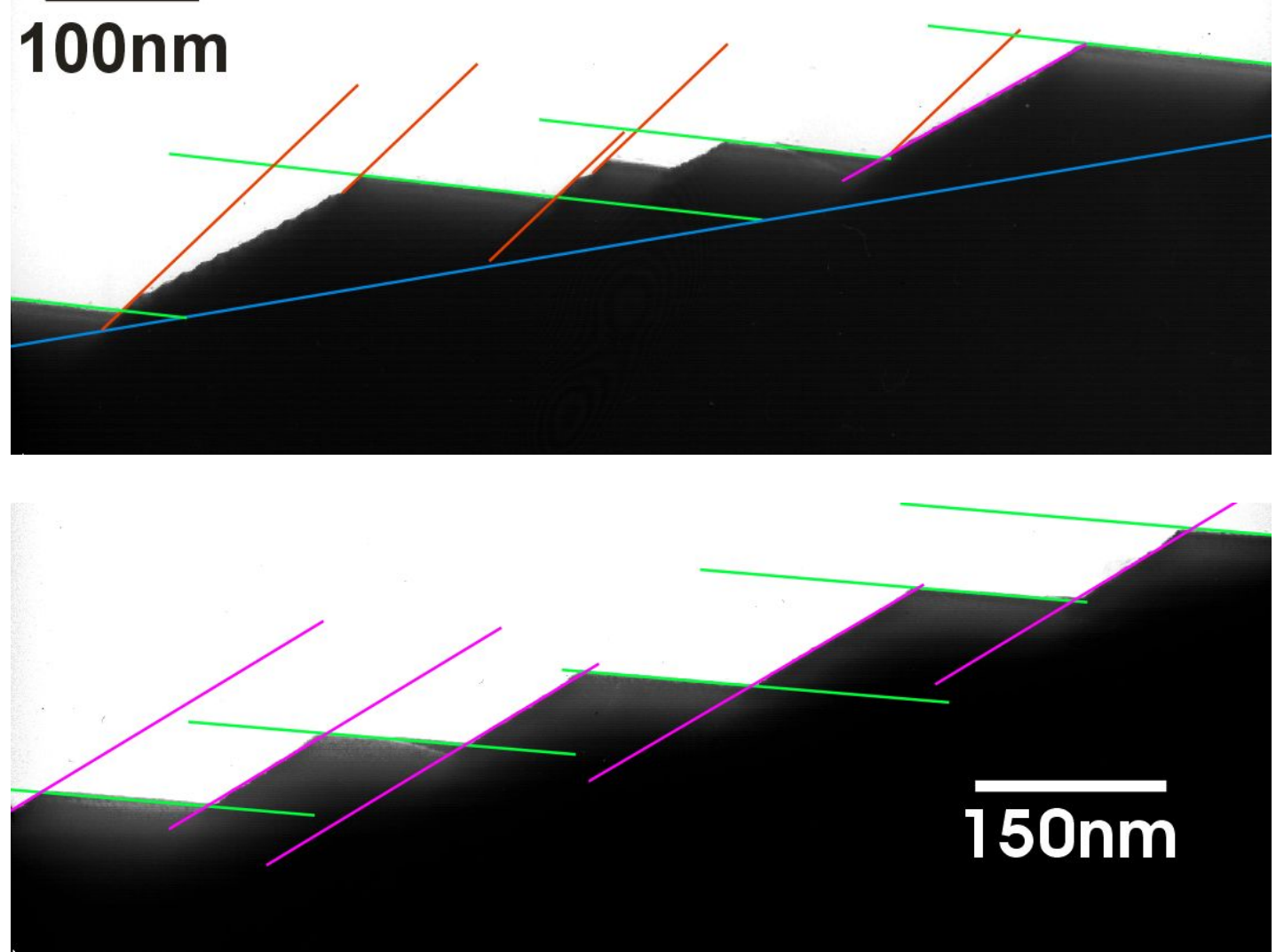

Abbildung 13: TEM-Querschnittsaufnahmen eines $24 \mathrm{~h}$ bei $1550^{\circ} \mathrm{C}$ getemperten Substrats;

Strahlrichtung entspricht der [1 $2 \overline{1} 0]$-Richtung; blaue Linie: parallel zur vormaligen Oberfläche, grüne Linien: $17,6^{\circ}$ zur blauen Linie, das entspricht der (10 111$)$-Kristallfläche; rote Linen 32,4 $4^{\circ}$ in entgegengesetztem Drehsinn zur blauen Linie, das entspricht der (10 $\overline{1} \overline{2})$-Kristallfläche; magentafarbene Linien: Facettenseite symmetrisch zur (10 $\overline{1} 1)$-Kristallflächenseite.

Abbildung 13 zeigt zu diesem Zweck zwei TEM-Querschnittsbilder der getemperten Saphiroberfläche. In den Abbildungen 13 und 15, sowie in der Skizze in Abbildung 14 wurden zur Verdeutlichung ausgewählte Kristallebenen durch farbige Linien gekennzeichnet. Blaue Linien entsprechen der ursprünglichen (10 $\overline{1} 0)$-Substratoberfläche, grüne und rote Linien entsprechen $(10 \overline{1} 1)$ - bzw. $(10 \overline{1} \overline{2})$-Ebenen, die einen Winkel von 17,6 $6^{\circ}$ bzw. 32,4 $4^{\circ}$ zur $(10 \overline{1} 0)$-Ebene aufweisen (vgl. auch Abbildung 14). Heffelfinger et al. geben eben diese Kristallflächen als Facettenseiten an [Hef 97]. Neben AFM-Untersuchungen zeigen aber auch die TEM-

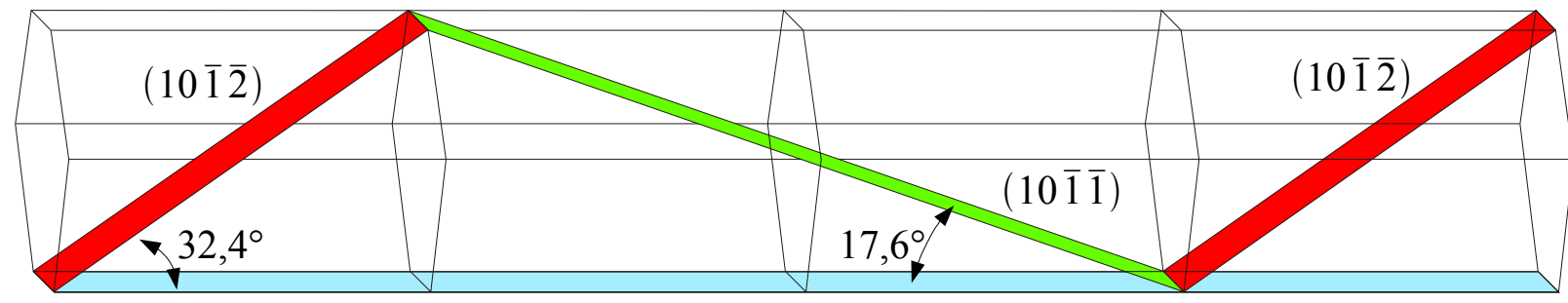

Abbildung 14: Orientierung der Facettenflächen bezüglich der hexagonalen Einheitszellen von $\alpha-\mathrm{Al}_{2} \mathrm{O}_{3}$. Die Farbkodierung entspricht der in Abbildung 13. 
Querschnittsabbildungen offensichtlich, dass die Facettierung nicht einfach als eine asymmetrische Anordnung dieser zwei niedrig indizierten Kristallflächen beschrieben werden kann.

Es besteht zusätzlich eine starke Tendenz zur Ausbildung einer symmetrischen Struktur. Während eine Facettenseite durch sehr glatte (10 11 ) -Flächen (grün) gebildet wird, weist die andere Seite einen komplizierteren Aufbau auf, so dass mesoskopisch eine zur Gegenseite symmetrische Facettenseite (magenta) mit eine Winkel nahe 17,6 $6^{\circ}$ zur ursprünglichen Oberfläche ausgebildet wird. Bei dieser Fläche handelt es sich um eine hochindizierte Kristallfläche. Abbildung 13 unten zeigt anhand eines typischen Bereichs, dass diese Fläche dennoch bei (fast) jeder Facette gebildet wird.

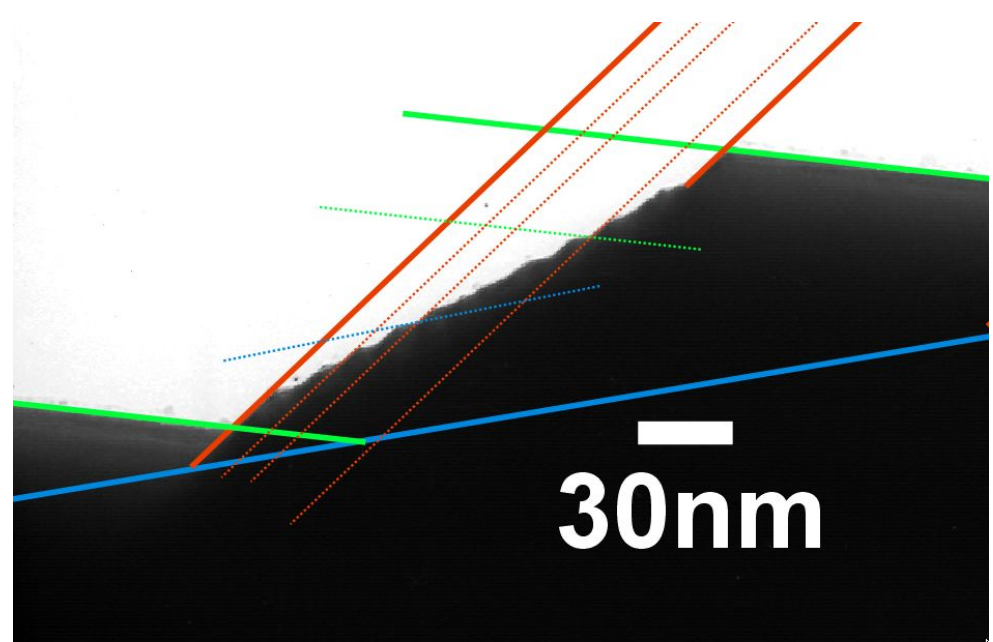

Abbildung 15: Nanofacettierte Seite (entsprechend den magentafarbenen Facettenseiten in Abbildung 13. Ausgedehnte (10 $\overline{1} \overline{2})$ Anteile an Kamm und Tal (rot) und als Nanofacettenseiten (rot gepunktet). Die Gegenseiten der Nanofacetten variieren in der Orientierung (grün gepunktet und blau gepunktet).
Um den Aufbau dieser Seite genauer aufzuzeigen, ist in Abbildung 15 ein stark vergrößerter Ausschnitt einer einzelnen entsprechenden Facettenseite dargestellt. Im Gegensatz zur sehr glatten $(10 \overline{1} 1)$-Seite weist diese Facettenseite eine gestufte, nanofacettierte Oberfläche auf. Auffällig sind größere Bereiche der von Heffelfinger et al. beschriebenen $(10 \overline{1} \overline{2})$-Kristallfläche im Bereich der Kämme und Täler. Die gesamte Seite besitzt große, jedoch nicht zusammenhängende Anteile dieser Fläche, die eine niedrigere Oberflächenenergie als die (10 $\overline{1} 0)$ Fläche hat [Sus 92]. Unterbrochen werden die Flächenbereiche von verschiedenen anderen Kristallflächen, u.a. $(10 \overline{1} 0)$ - (blau gepunktet) und (10 $\overline{1} 1)$-Flächen (grün gepunktet), wodurch diese Facettenseite in Nanofacetten zerfällt und mesoskopisch eine symmetrische Struktur möglich wird. Die Nanofacettierung deutet nicht zwangsläufig auf weitere treibende Kräfte hin, sie kann auch einer Konfiguration minimaler Oberflächenenergie entsprechen. Auf die Energetik facettierter bzw. gestufter Oberflächen wird im folgenden Kapitel näher eingegangen. 
Die Ergebnisse aus den Untersuchungen der Frühstadien der Facettierung mit Rasterkraftmikroskopie zusammen mit den Aussagen, die aufgrund der TEMQuerschnittspräparation erzielt werden konnten, ergeben folgendes Bild:

Aus dem unsymmetrischen Frühstadium der Facettierung, in dem die Facettenseiten stark unterschiedlich sind, entwickeln sich bis zum Endstadium weitgehend symmetrische Facetten einer Periodizitätslänge von ca. 320nm und einer Höhe von ca. 50nm. Beide Facettenseiten schließen mit der vormaligen Substratoberfläche einen Winkel von $17,6^{\circ}$ ein. Dazu vergrößert sich die im Frühstadium bereits beobachtete, glatte Facettenseite, die als (10 $\overline{1} 1)$ Kristallfläche indiziert werden konnte, auf die volle Facettenseitenbreite. Die bereits

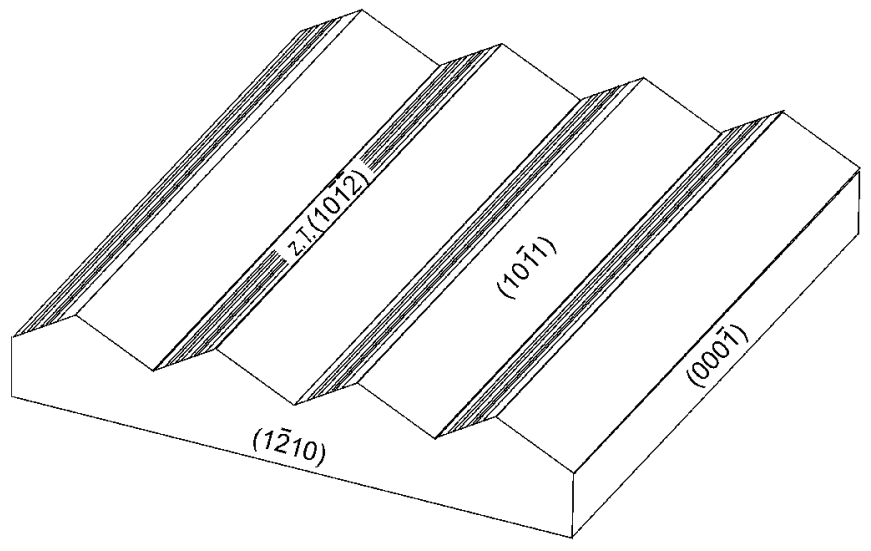

Abbildung 16: Kristallographische Orientierung der Facettenseiten. Während die eine Facettenseite der (10 $10 \overline{1}$ 1) -Kristallfäche entspricht, ist die andere Seite aus Nanofacetten mit (10 1012$)$-Flächen sowie wechselnden Orientierungen aufgebaut. Facettierungsrichtung ist die

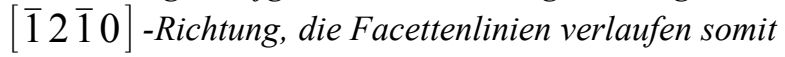
senkrecht zur c-Achse (0001). im Frühstadium vorhandene korrelierte Rauigkeit der anderen Facettenseite, ist durch Nanofacetten zu erklären, deren Facettenseiten aus $(10 \overline{1} \overline{2})$-Kristallflächen und Gegenseiten verschiedener Orientierungen bestehen, die somit den symmetrischen Facettierungswinkel der übergeordneten, mesoskopischen Facettenseite von 17,6 $6^{\circ}$ einstellen können. Am Facettental und -kamm sind schon früh Ansätze der $\{10 \overline{1} \overline{2}\}$-Fläche erkennbar.

Die Morphologie und Orientierung der facettierten m-plane $\mathrm{Al}_{2} \mathrm{O}_{3}$-Fläche lässt sich entsprechend Abbildung 16 zusammenfassend darstellen.

\subsection{Energetik der Facettierung}

Die starken morphologischen Veränderungen, die bei der Facettierung einer niedrig indizierten Oberfläche wie der $\mathrm{Al}_{2} \mathrm{O}_{3}$ (10 $\left.\overline{1} 0\right)$-Kristallfläche durch einfache Temperung auftreten, erscheinen zunächst kontraintuitiv. Im Allgemeinen zeichnen sich niedrig indizierte Flächen durch eine hohe Stabilität aus (insbesondere bei Metallen). Im Folgenden werden kurz die wichtigsten Aspekte zur Oberflächenenergie dargestellt, sowie in komprimierter Form die wichtigsten Energiebeiträge einer facettierten Oberfläche erwähnt. Für ausführliche und umfassende Darstellungen, sowie Grundlagen aus dem Bereich wird auf Lehrbücher der Oberflächenphysik, wie z.B. [Hen 94] und [Pim 98] verwiesen. Eine detaillierte Darstellung der thermodynamischen Beschreibung von vizinalen und facettierten Oberflächen findet sich in [Shc 95] und [Shc 99].

Die Minimierung der Oberflächenenergie $\gamma$ durch Bildung von Facetten mit niedrigerer Oberflächenenergie als die der ursprünglichen Oberfläche ist die erste Triebkraft für die Veränderung der Oberfläche. Während bei Metallen die Oberflächenenergien für unterschiedliche Kristallebenen nur wenig voneinander abweichen, können diese bei Keramiken erhebliche Differenzen aufweisen [Sus 92]. Die Oberflächenenergie für kfz-Metalle variiert in den Verhältnissen $\gamma_{(111)}: \gamma_{(100)}: \gamma_{(110)} 1: 1,047: 1,150$. Betrachtet man hingegen $\alpha-\mathrm{Al}_{2} \mathrm{O}_{3}$, so hat die 
relaxierte $(10 \overline{1} 2)$-Oberfläche mit 2,57 $\mathrm{Jm}^{-2}$ die niedrigste Oberflächenenergie. Diese ist damit weniger als halb so groß, wie die der $(10 \overline{1} 0)$-Oberfläche mit $6,87 \mathrm{~J} \mathrm{~m}^{-2}$. Als Folge davon sollte der Habitus von $\alpha-\mathrm{Al}_{2} \mathrm{O}_{3}$ rhomboedrisch sein. Es ergeben sich die Verhältnisse $\gamma_{(10 \overline{1} 2)}: \gamma_{(10 \overline{1} 1)}: \gamma_{(10 \overline{1} 0)}: \gamma_{(0001)}: \gamma_{(10 \overline{1} 4)}$ zu 1:1,27:2,67:1,16:3,90. [Tas 88].

Verschiedene Autoren haben die Oberflächenenergien für die unterschiedlichen Kristallebenen in $\alpha-\mathrm{Al}_{2} \mathrm{O}_{3}$ berechnet [Wul 01], [Mar 81], [Sus 92], [Mad 94]. Die ideale Kristallform (equilibrium crystal shape, ECS) kann durch die Wulff-Konstruktion ermittelt werden, bei der unter Voraussetzung der Konstanz des Kristallvolumens die Gesamtoberflächenenergie des Kristalls

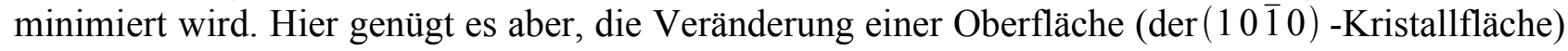
$\mathrm{zu}$ betrachten, da diese im vorliegenden Fall von Substraten für die Dünnschichtherstellung gegenüber den restlichen Seiten sehr groß ist.

Grundsätzlich unterscheidet man die Terrassen-Stufen-Morphologie und die facettierte Oberfläche. In beiden Fällen ist der Grund für die Oberflächenänderung die Minimierung der gesamten Oberflächenenergie.

(1)Terrassen-Stufen-Morphologie: Ein Einkristall, der geringfügig $\left(0,1^{\circ}\right.$ bis einige Grad) aus einer niedrig-indizierten Richtung verkippt ist, bildet in der Regel eine Oberfläche aus breiten Terrassen der zugehörigen niedrig indizierten Kristallfläche aus, die durch (teilweise nur atomare Stufen voneinander getrennt sind. Man spricht von vizinalen Oberflächen.

(2)Facettierte Oberfläche: Diese Morphologie wurde von Mullins 1961 als „hill-and-valley“ beschrieben [Mul 61]. Sie entsteht während Temperungen, wenn die terminierende (niedrigindizierte) Kristallfläche eine geringere Stabilität - also eine höhere Oberflächenenergie aufweist, als die sich bildenden Oberflächen der dachartigen Facetten.

Eine detaillierte Darstellung und Unterscheidung von vizinalen und facettierten Flächen findet sich z.B. bei Heffelfinger et al. [Hef 97] und Ramamurthy et al. [Ram 00].

An dieser Stelle sollen nur kurz die wichtigsten Beiträge zur freien Energie einer facettierten Oberfläche erwähnt werden.

Geht man davon aus, dass bei der Facettierung die Gesamtorientirung $\boldsymbol{n}$ erhalten bleibt $\left(\boldsymbol{n}=\alpha \cdot \boldsymbol{n}_{\mathbf{1}}+\beta \cdot \boldsymbol{n}_{\mathbf{2}}\right.$ $\boldsymbol{n}_{\boldsymbol{i}}$ sind die Ebenennormalen der Facettenseiten), so ist die notwendige Bedingung für den Zerfall eine Reduktion der Oberflächenenergien.

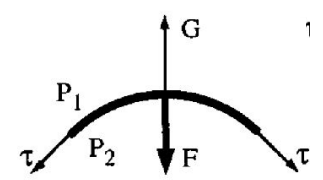

Abbildung 17: Spannungsfeld: Analogie von Flüssigkeit und Facetten des Festkörpers (aus [Shc 99]).

$$
\gamma_{n}>\alpha \cdot \gamma_{n_{1}}+\beta \cdot \gamma_{n_{2}}
$$

Gleichung 1

Hierbei berücksichtigen die Faktoren $\alpha$ und $\beta$ verschiedene Flächenanteile der Facettenseiten. Die auf die Fläche bezogene freie Energie sinkt damit um den Beitrag $\Delta F_{1}=\Delta \gamma$. Tasker [Tas 88] gibt für die $(10 \overline{1} 0)$-Oberfläche eine Oberflächenenergie von $6,87 \mathrm{Jm}^{-2}$ an, während die der Facetten mit $(10 \overline{1} 1)$ - bzw. $(10 \overline{1} \overline{2})$-Orientierung 3,27 bzw. 2,57 Jm ${ }^{-2}$ betragen. Es ist sofort einsichtig, dass eine Halbierung der Oberflächenenergie die Triebkraft für die Bildung der günstigeren Kristallebenen sein kann. Dabei ist diese unabhängig von der Facettierungsperiode $D$. Dieser Beitrag kann also nicht das Auftreten einer periodischen Anordnung der Facetten erklären, prinzipiell könnte eine Kristalloberfläche auch in zwei einzelne Superfacettenseiten zerfallen. Hierbei ist zu berücksichtigen, dass Facettentäler und -kämme aufgrund der veränderten Symmetrie 
als Störungen der glatten Fläche durch Facettenlinien mit der Selbstenergie $\eta=\eta_{\text {Tal }}+\eta_{\text {Kamm }}$ aufgefasst werden können. Eine die Facetten begrenzende Anordnung von Tälern und Kämmen erhöht somit die auf die Fläche bezogene Energie um den Beitrag:

$$
\Delta F_{2}=\frac{\eta_{\text {Tal }}+\eta_{\text {Kamm }}}{D}
$$

Gleichung 2

Mit diesen Facettenlinien ist aber auch die Existenz eines langreichweitigen Spannungsfeldes verbunden, dessen Ursprung analog zum inneren Druck eines Flüssigkeitströpfchens ist (vgl. Abbildung 17).

Dieses auch im thermodynamischen Gleichgewicht vorhandene Spannungsfeld verringert die Oberflächenenergie an Kristallkanten. Nach Marchenko [Mar 81] reduziert sich die flächenbezogene freie Energie einer periodischen Anordnung von Facettenlinien um den Beitrag:

$$
\Delta F_{3}=-\frac{K}{D} \ln \left(\frac{D}{a}\right)
$$

Gleichung 3

Fasst man diese drei Beiträge zur Absenkung der freien Energie zusammen, so erkennt man, dass der maximalen Absenkung eine im Wesentlichen durch Materialkonstanten ( $a$ ist die Gitterkonstante, $K$ beinhaltet nach Marchenko u.a. den Elastizitätsmodul) bestimmte optimale Periodenlänge $D_{o p t}$ entspricht (siehe Abbildung 18).

$$
D_{\text {opt }}=a \cdot \exp \left[\frac{\eta}{K}+1\right]
$$

Gleichung 4

Die Konfigurationsentropie der Abweichung von der periodischen Anordnung der Facetten ist in dieser vereinfachten Darstellung nicht berücksichtigt, wird von Shchukin et al. für eine makroskopisch facettierte Oberfläche aber als vernachlässigbar eingeschätzt [Shc 95].

Ein wesentlicher Unterschied zwischen den facettierten und den vizinalen Oberflächen bei der Betrachtung der elastischen Wechselwirkung ist, dass bei vizinalen Flächen die an einer Stufenkante vorliegenden Kräfte bezüglich der elastischen Fernwirkung als Dipole angesehen werden können, $\mathrm{da}$ die Terrassenhöhe $\mathrm{h}$ deutlich kleiner als die Terassenbreite $D(\approx L)$ ist (vgl. Abbildung 19). Dabei können Stufen einer vizinalen Oberfläche nach Marchenko et. al als Reihe von Punktdipolen auf der nominellen Oberfläche angesehen werden,

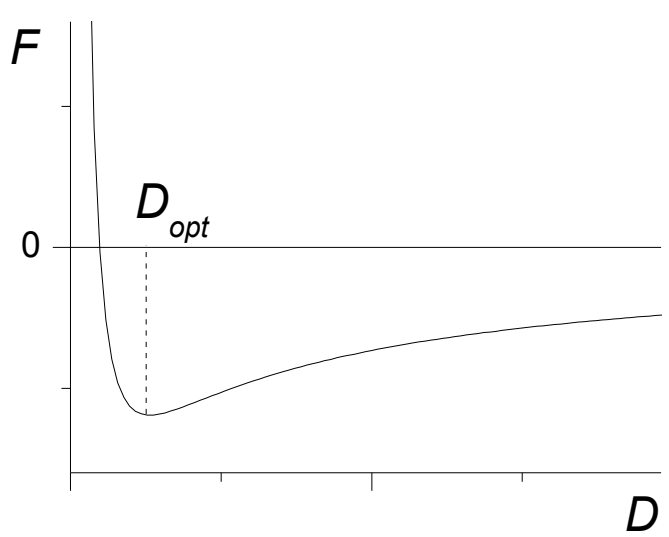

Abbildung 18: Optimale Facettierungsperiode $D_{\text {opt }}$ aus der minimalen freien Energie. die sich über die Dipol-Dipol Wechselwirkung $\left(\propto D^{-2}\right)$ ordnen [Mar 80].

Viele experimentelle Ergebnisse haben jedoch gezeigt, dass hiermit nicht alle Morphologien erklärbar sind. Prévot et al. schlagen daher ein „,burried dipol model“ vor, das es erlaubt, auch spezielle Stufengeometrien $\mathrm{zu}$ berücksichtigen und anisotrope Elastizitätsrechnungen durchzuführen. Hier finden dann Terme höherer Ordnung (z.B. $\propto D^{-3}$ ) Einfluss im 
Wechselwirkungsmechanismus, die sehr gut mit MD-Simulationen übereinstimmen und weitere Experimente erklären können [Pre 04].

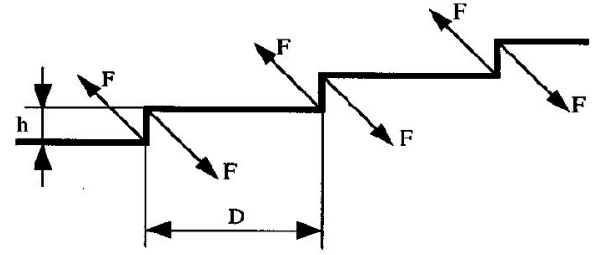

Abbildung 19: Kräftedipole an den Stufen einer vizinalen Oberfläche (aus [Shc 99]).

\subsection{Kinetik der Facettierung}

Neben der Orientierungsbestimmung und Analyse des Endzustands ist es von Interesse, aus den Zwischenzuständen im Verlauf der Morphologieänderung Hinweise auf den Ablauf, den Materialtransport und damit auf die Kinetik der Facettierung zu erhalten.

\section{a) Keimbildung}

Die großflächige Untersuchung von Substraten, die bei nur $1300^{\circ} \mathrm{C}$ getempert wurden, zeigt, dass die Facettierung in verschiedenen Bereichen unterschiedlich weit fortgeschritten ist. Während sich einerseits Gebiete mit klaren, geradlinigen Facettenkämmen und -tälern wie in Abbildung 10 finden, ist in anderen Bereichen die Morphologie verändert: Die Höhendifferenzen sind reduziert, die Facettierungslinien sind aufgebrochen (siehe Abbildung 20). Die Verteilung der Zentren, von denen aus die Facettierung fortschreitet, ist statistisch. Daher kann an diesen Orten die Veränderung der Oberfläche im Sinne einer homogenen Keimbildung auf Höhenfluktuationen beginnen. Eine

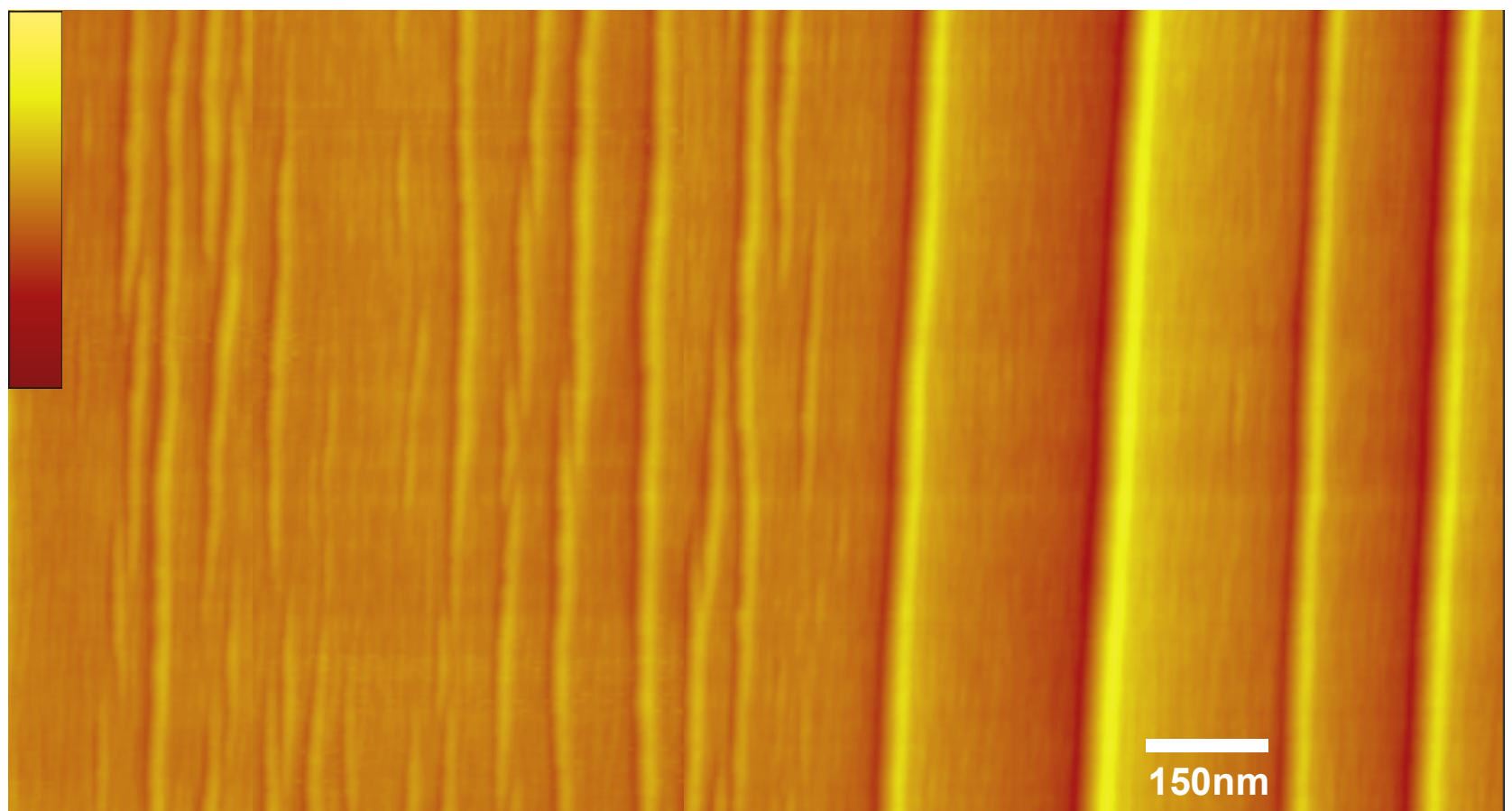

Abbildung 20: AFM-Höhendaten: 1,9 $\mathrm{mm}$ x $1 \mu \mathrm{m}$ Bildbereich, Temperung $24 \mathrm{~h}$ bei $1300^{\circ} \mathrm{C}, \mathrm{z}$-Skala: 20nm. Rand einer Facettendomäne. Ausbildung einer geraden, gemeinsamen Facettenlinie durch Aufstauen kleinerer Facetten.

heterogene Keimbildung, d.h. der Beginn des Wachstums von Facetten an Oberflächenveränderungen wie Kratzern, ist sicher auch möglich, jedoch wurde von Heffelfinger et al. [Hef 97] 
beobachtet, dass typische Defekte die durch die Politur des Substrats eingebracht wurden, ausheilen und die mittlere Rauigkeit (RMS) durch kurze Auslagerungen (5min) bei „moderaten“ Temperaturen von $1400^{\circ} \mathrm{C}$ um mehr als eine Größenordnung $(2,1 \mathrm{~nm}$ auf $0,2 \mathrm{~nm})$ reduziert wird. Nur Oberflächenveränderungen, die diese Glättungsphase überdauern, können als heterogene Keime für das Facettenwachstum fungieren. Die gleichmäßige Verteilung der in einem späteren Stadium der Facettierung $\mathrm{zu}$ beobachtenden Facettierungsdomänen deutet ebenfalls auf eine dominierende homogene Keimbildung hin.

\section{b) Wachstum}

Aspekte zum Wachstum von Facetten liefert die Betrachtung des Aufbaus einer einzelnen Facette über ihre gesamte Länge. Das variierende Verhältnis von Facettenkammhöhe und Facettentaltiefe entlang der Facettenlinie lässt Rückschlüsse auf eine bevorzugte Materialtransportrichtung, also auf Materialquellen und -senken für die morphologischen Veränderungen während der Facettierung zu.

Der in der AFM-Höhenabbildung und Schnittbildabfolge in Abbildung 21 magentafarben markierte Bereich umfasst eine einzelne Facette. In der Mitte ist die ausgebildete (10 $\overline{1} 1)$-Facettenseite durch ein tiefes Tal begrenzt, wohingegen an den Spitzen der Facettenlinie (Abbildung 21b Schnittlinie 1 und 5) praktisch nur noch eine Erhebung, aber kein Tal neben der Facette erkennbar ist. Diese Beobachtung legt nahe, dass das Facettenwachstum durch Oberflächendiffusion entlang der Facettenlinie (in $[1 \overline{2} 10]$ - resp. [ $\overline{1} 2 \overline{1} 0]$-Richtung) mit Materialtransport von der Mitte an die Spitzen erfolgt.

a)

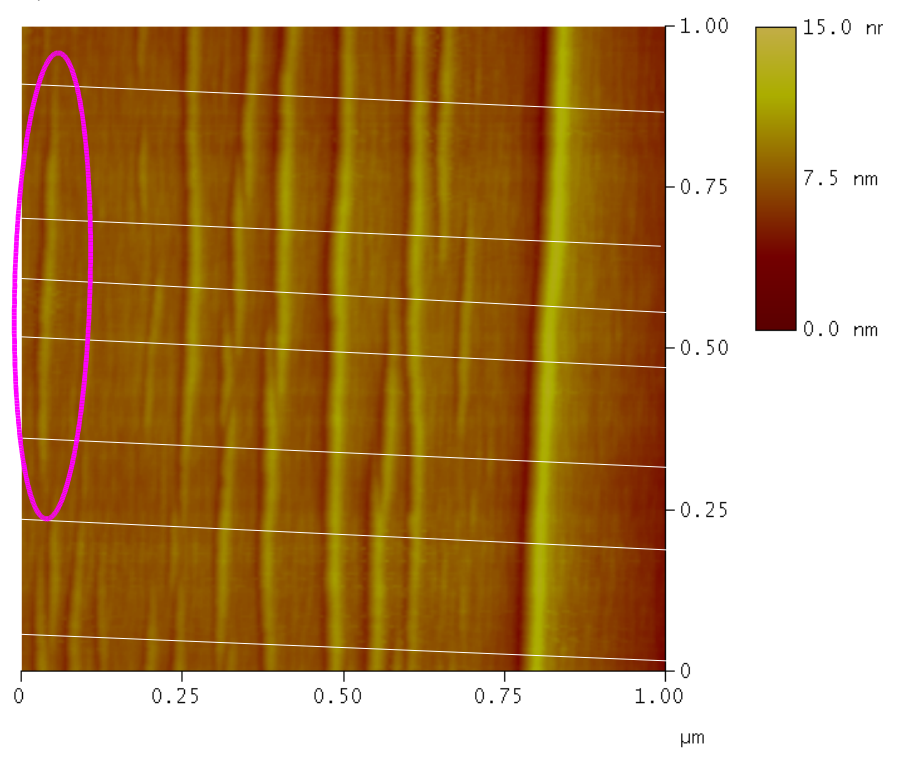

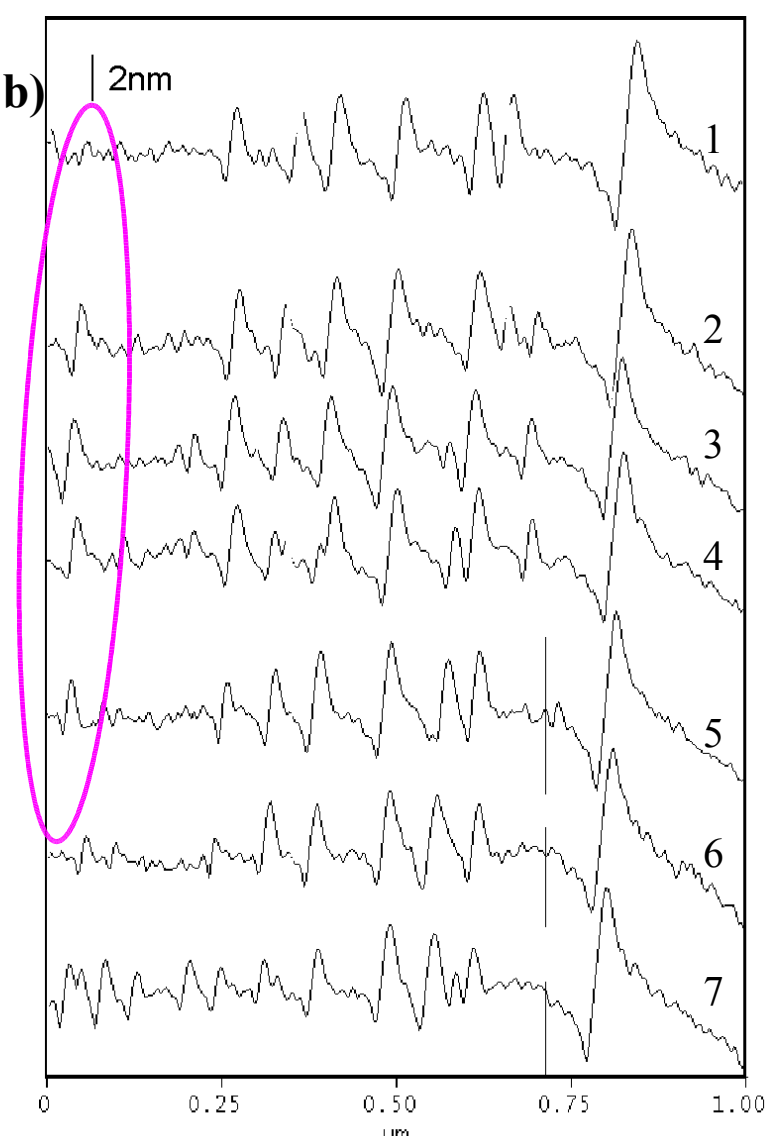

Abbildung 21: a) AFM-Höhendaten und b) mehrfache Schnittdarstellung. $1 \mu m^{2}$ Bildbereich eines $24 \mathrm{~h}$ bei $1300^{\circ} \mathrm{C}$ getemperten Substrats. Die weißen Linien in Abbildung a) entsprechen den Positionen der Schnitte. Die magentafarbene Ellipse markiert eine einzelne Facettenlinie und den entsprechenden Bereich in der Schnittbildabfolge.

Neue Facetten nukleieren bevorzugt direkt neben bereits vorhandenen Facetten. Der Materialabtransport im mittleren Bereich einer Facette, der als Materialquelle fungiert, führt zur 
starken Oberflächenkrümmung. Diese Stellen dienen als Nukleationspunkte für die Bildung neuer Facetten. Dabei fällt auf, dass diese Nukleation besonders auf Seiten der glatten (10 11$)$ Kristallfläche erfolgt. Hier sind die Veränderungen der Oberfläche durch das Facettenwachstum am stärksten.

\section{c) Domänenbildung und -wachstum}

Diese bevorzugte Keimbildung führt zu ersten Vorstufen von Facettierungsdomänen. Die Domänen vergrößern sich in Facettierungsrichtung (in [1 $\overline{2} 10]$ - bzw. [1 $2 \overline{1} 0]$-Richtung) durch das Weiterwachsen der einzelnen Facetten, wohingegen die Ausdehnung senkrecht zur Facettierungsrichtung (in $\left[\begin{array}{llll}0 & 0 & 0 & 1\end{array}\right]$ - resp. $\left[\begin{array}{llll}0 & 0 & 0 & \overline{1}\end{array}\right]$-Richtung) durch Bildung weiterer Facetten an den Seiten der äußersten Facetten erfolgt.

Die Ausbildung von stabilen Facetten geht einher mit einem Vergröberungsprozess. Untersuchungen von Proben, die bei niedrigen Temperaturen getempert wurden, zeigen, dass durchgehende Facettenkämme nur bei großen Facetten vorhanden sind (siehe Abbildung 20). Kleinere Nukleationsfacetten in den Randbereichen der Facettendomänen schließen sich offensichtlich durch Aufstauung zu größeren zusammen. Bildet sich eine gemeinsame Facettenlinie, so reduziert sich die Zahl der Nanofacetten auf der flachen Facettierungsseite und die Amplitude nimmt zu (Abbildung 23). Diese Beobachtungen sprechen dafür, dass während des Wachstums der Domänen neben dem Wachstum in Facettierungsrichtung auch eine langsame Bewegung der Facettenlinie senkrecht zur Facettierungs-

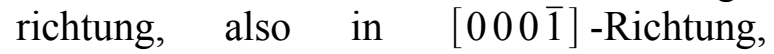
stattfindet, bei der sich kleine Nukleationsfacetten aufstauen.

Auch für die Transportvorgänge in diese „langsame“ Richtung finden sich Hinweise zur Kinetik in den Daten der Rasterkraftmikroskopie. In allen Stadien werden sehr glatte $(10 \overline{1} 1)$-Facettenseiten beobachtet. Des weiteren sind Einkerbungen am Fuß und Überhöhungen am Kamm dieser Seiten $\mathrm{zu}$ finden (siehe Abbildung 22). Diese charakteristischen Überhöhungen an den (10 $\overline{1} 1)$-Facettenseiten deuten auf einen sehr schnellen Eigentransport auf dieser Kristallfläche hin. Der An- bzw. Abtransport von Material auf der flachen, rauen Facettenseite ist offensichtlich deutlich langsamer, so dass es zu Verarmungszonen am Fuß bzw.

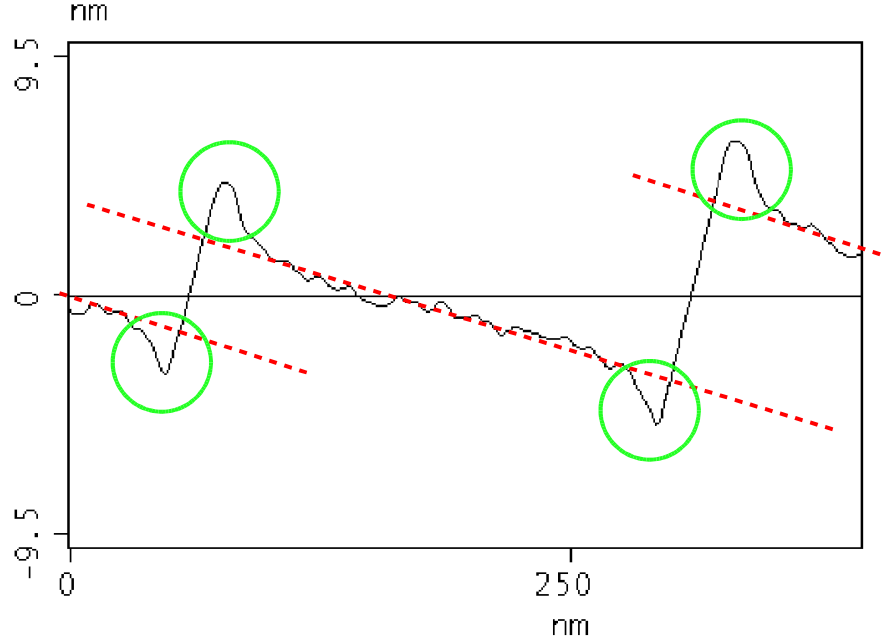

Abbildung 22: Schnittdarstellung aus AFM-Höhendaten; Temperung: $24 \mathrm{~h}$ bei $1300^{\circ} \mathrm{C}$. Rot unterbrochene Linien zeigen den mittleren Verlauf der Facettenseite, grüne Kreise markieren Einkerbung (Tal) bzw. Überhöhung (Kamm). Überschusszonen am Kamm kommt. Die sich ausbildenden Ansätze größerer Steigung begünstigen die Bildung der $\{10 \overline{1} \overline{2}\}$-Kristallflächen, weshalb an den Ansätzen der nanofacettierten Facettenseite nach vollständiger Facettierung ausgedehntere Bereiche dieser Orientierung zu finden sind (siehe Abbildung 15).

Transportvorgänge in $\alpha-\mathrm{Al}_{2} \mathrm{O}_{3}$ sind aufgrund der hohen technologischen Bedeutung des Materials sehr intensiv untersucht worden; viele Resultate und die zugrunde liegenden Mechanismen sind aber nach wie vor weitgehend unverstanden [Atk 88], [Tso 94]. Es ist bekannt, dass die Bildung von $\mathrm{Al}_{2} \mathrm{O}_{3}$-Randschichten durch Diffusion von Aluminium an die Oberfläche und nachfolgender Oxidation erfolgt, im Gegensatz zur $\mathrm{SiO}_{2}$-Passivierung bei der Sauerstoff von außen eindiffundiert [Yos 04]. Für $\mathrm{Al}_{2} \mathrm{O}_{3}$ ist jedoch im Temperaturbereich von $1273 \mathrm{~K}$ bis $1763 \mathrm{~K}$ (also $\sim 1 / 2 \cdot \mathrm{T}_{\mathrm{m}}$ bis $3 / 4 \cdot \mathrm{T}_{\mathrm{m}}$ ) 
die Oberflächendiffusion gegenüber der Volumendiffusion der dominante Transportmechanismus [Tso 96]. Diese wird durch die bekannte Arrhenius-Abhängigkeit (Gleichung 4) beschrieben.

$$
D=D_{0} \cdot e^{-\frac{Q_{\text {diff }}}{R \cdot T}}
$$

Gleichung 5

Tsoga et al. ermittelten für polykristallines $\mathrm{Al}_{2} \mathrm{O}_{3}$ den präexponentiellen Faktor $D_{0}=0,48 \mathrm{~cm}^{2} / \mathrm{s}$ und eine Aktivierungsenergie für die Oberflächendiffusion von $Q_{\text {diff }}=256 \mathrm{~kJ} / \mathrm{mol}$. Bei Sinterexperimenten bei nur leicht höheren Temperaturen (bis $0,8 \mathrm{~T}_{\mathrm{m}}$ ) wurden Aktivierungsenergien bis zu $Q_{\text {diff }}=519 \mathrm{~kJ} / \mathrm{mol}$ gemessen [Saj 87]. Bei oxidischen Festkörpern, wie $\mathrm{Al}_{2} \mathrm{O}_{3}$ kann es durch den ionischen Charakter der Struktur zu diesen hohen Aktivierungsenergien kommen.

Insbesondere der Transport auf speziellen kristallographischen Ebenen von $\alpha-\mathrm{Al}_{2} \mathrm{O}_{3}$ ist ein komplexer Prozess. $\mathrm{Da}$ die untersuchte Facettierung eine sauerstoffreiche Umgebung erfordert, müssen möglicherweise sogar kombinierte Prozesse, wie Sauerstoffdesorption, diffusiver Transport der Aluminiumionen mit nachfolgender Sauerstoffresorption, berücksichtigt

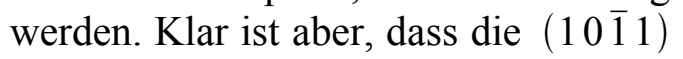
-Seite einen schnellen Transport senkrecht zur Facettierungsrichtung begünstigt. Hier ist also die Kinetik (Oberflächendiffusion) und nicht die Thermodynamik dafür verantwortlich, dass sich die (10) 11$)$-Fläche grossflächig glatt ausbildet im Gegensatz $\mathrm{zu}$ der nanofacettierten $(10 \overline{1} \overline{2})$-Seite. Da der Facettierungswinkel für die letztgenannte Seite mit $32,4^{\circ}$ auch deutlich über dem der (10 1011$)$-Seite liegt, ist zur Ausbildung einer zusammenhängenden Fläche

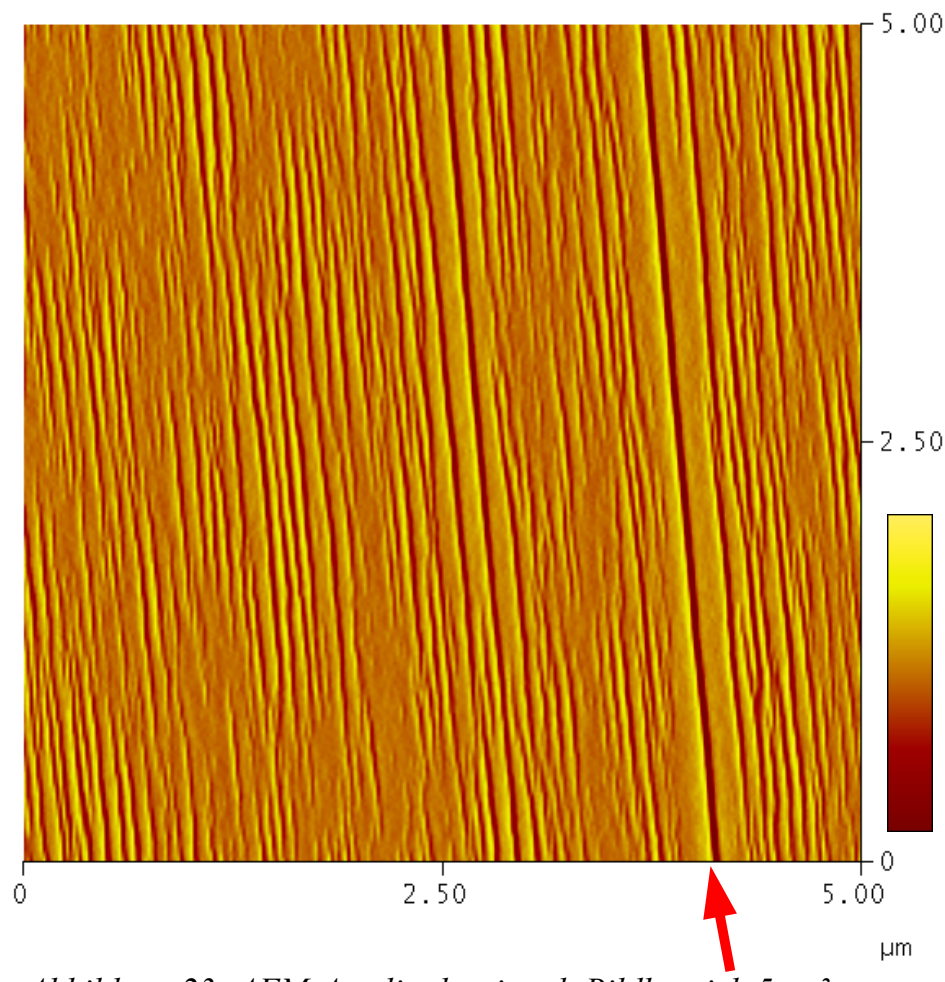

Abbildung 23: AFM-Amplitudensignal, Bildbereich $5 \mu^{2}$, $z$-Skala $=0,4 \mathrm{~V}$, Temperung: $24 \mathrm{~h}$ bei $1300^{\circ} \mathrm{C}$; Der Pfeil markiert eine durchgehende Facettenlinie durch gleiche laterale Positionen in verschiedene Domänen.

dieser Orientierung ein deutlich größerer Massetransport erforderlich.

\section{d) Bildung von Domänengrenzen}

Das Wachstum in Facettierungsrichtung wird durch benachbarte Facettierungsdomänen begrenzt. Abbildung 23 veranschaulicht ein Stadium, in dem verschiedene Domänen aufeinandertreffen. Wachsen zwei Facettendomänen in Facettierungsrichtung zusammen, kommt es an der Kontaktlinie in der Regel zu einer großen Zahl von Fehlern. Zwar haben die unterschiedlichen Domänen eine sehr ähnliche durchschnittliche Facettenbreite, aber die laterale Position der Facettenkämme bzw. -täler (senkrecht zur Facettierungsrichtung) ist zufällig. Somit kommt es an den Schnittlinien von Facettendomänen zu „Stapelfehlern“ (siehe z.B. Abbildung 25 insbesondere oben links). Passt die laterale Position von Facettenlinien, so bildet sich eine durchgehende Linie und die Facettenhöhe 
wächst (siehe Abbildung 23). Rechts neben der markierten Linie ist eine störungsfreie Zone erkennbar, deren Breite nahezu der endgültigen Facettenbreite entspricht. Das Wachstum der Facetten in die Höhe bedingt die Reduktion der Facettenanzahl.

\section{e) Ordnungseinstellung und Ausheilen von Facettierungsdefekten}

Durch Steigerung der Temperatur auf über $1300^{\circ} \mathrm{C}$ ist unverzüglich eine Morphologie erreicht, in der die gesamte Probe von Facetten bedeckt ist. Abbildung 24 zeigt eine Bilderfolge für Temperaturen zwischen $1300^{\circ} \mathrm{C}$ und $1550^{\circ} \mathrm{C}$. Dargestellt sind jeweils $5 \mu \mathrm{m}$ große Bereiche. Die Höhenskala umfasst mit Ausnahme der Probe bei $1300^{\circ} \mathrm{C} 50 \mathrm{~nm}$. In Fall der Temperung bei nur $1300^{\circ} \mathrm{C}$ ist sie auf $20 \mathrm{~nm}$ reduziert, um die Oberflächenmorphologie noch sichtbar zu machen. Bis zu einer Temperatur von $1400^{\circ} \mathrm{C}$ wächst die maximal beobachtete Facettenhöhe an. Die Periodizität stellt sich bis zu einer Temperatur von $1375^{\circ} \mathrm{C}$ ein, d.h. die Facettenbreite gleicht sich an. Für die weiteren Untersuchungen ist es wichtig, Substrate herzustellen, deren Facettierungshöhe homogen ist, d.h. möglichst geringe Unterschiede in der Höhe der einzelnen Facetten bestehen und die möglichst lange durchgehende Facettierungslinien aufweisen. Durch die Temperung bis zu einer Temperatur von $1550^{\circ} \mathrm{C}$ gelingt eine weitgehende Reduktion der Facettierungsfehler, so dass die mittlere defektfreie Länge einer Facette eines vollständig facettierten Substrats aus SEMUntersuchungen mit größer $100 \mu \mathrm{m}$ abgeschätzt werden kann.

Die treibenden Kräfte für die Facettierung, der Einfluss der Facettenkämme und -täler auf die freie Energie $F$ durch ihre elastische Wechselwirkung, verringern sich mit steigender optimaler Facettierungsperiode $D_{\text {opt }}$. Die Tiefe des Minimums von $F$ bei $D_{\text {opt }}$, (siehe Abbildung 18) bzw. die lokale Steigung von $F$ bei $D_{\text {opt }}$ bestimmt, ob eine breite Verteilung oder aber eine scharf definierte Periodizität beobachtet wird (Gleichung 2). Ist die Facettierungsperiode im Bereich der Gitterkonstante $\left(D_{o p t} \approx a\right)$ ist eine einheitliche Periodizität $\mathrm{zu}$ erwarten (wie sie für vizinale Oberflächen auch beobachtet wird), im Fall der Saphir (10 10 ) -Fläche ist das Minimum aber flach, da $D_{\text {opt }}>>a$ ist, weshalb lange Temperzeiten bzw. hohe Temperaturen notwendig sind, um möglichst regelmäßige Facettenanordnungen zu erzeugen. 


\section{$1300^{\circ} \mathrm{C}$}

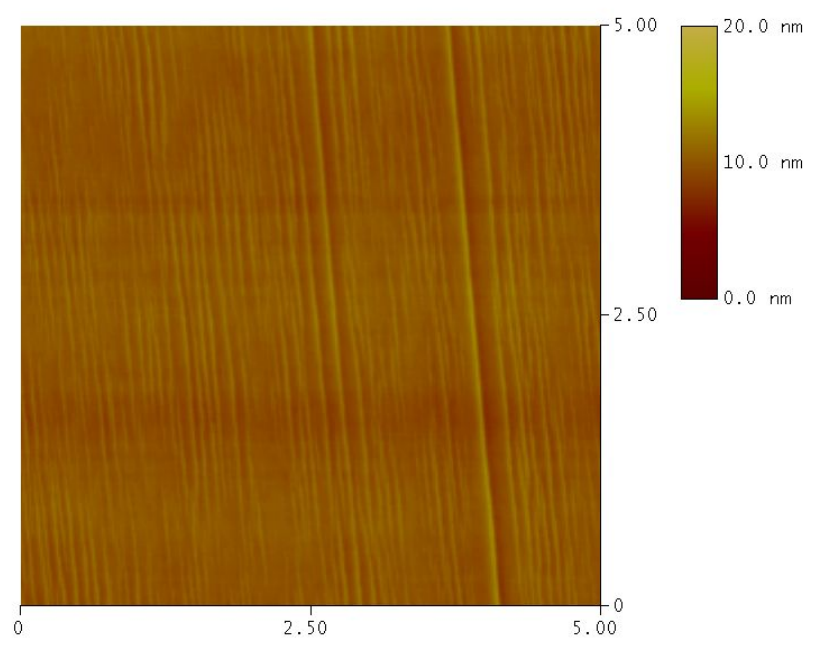

$1375^{\circ} \mathrm{C}$

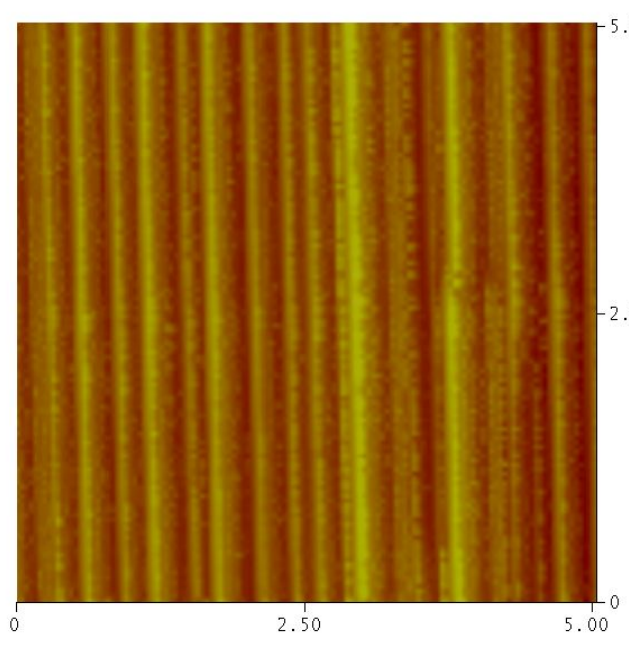

$1400^{\circ} \mathrm{C}$
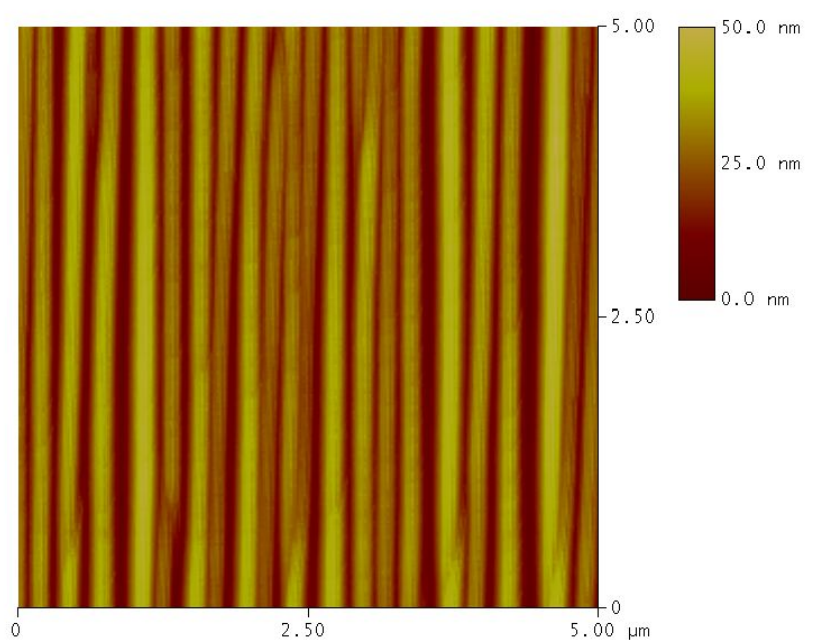

$1350^{\circ} \mathrm{C}$

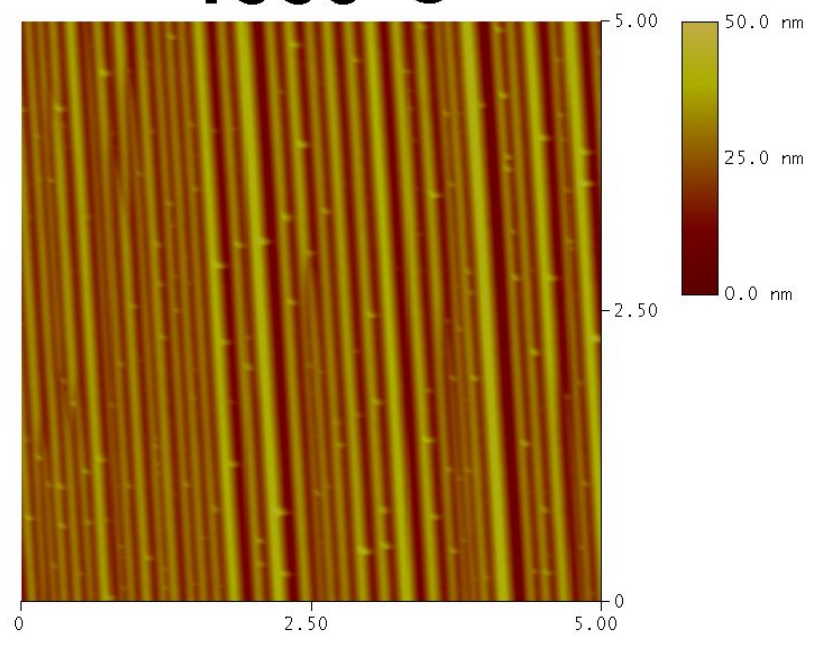

$1375^{\circ} \mathrm{C}$

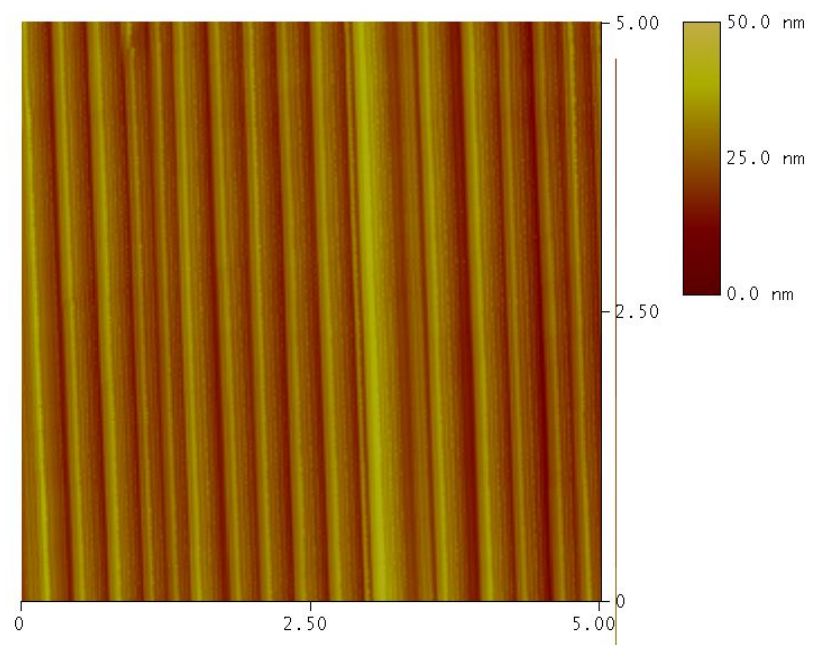

$1550^{\circ} \mathrm{C}$

$\mu \mathrm{m}$

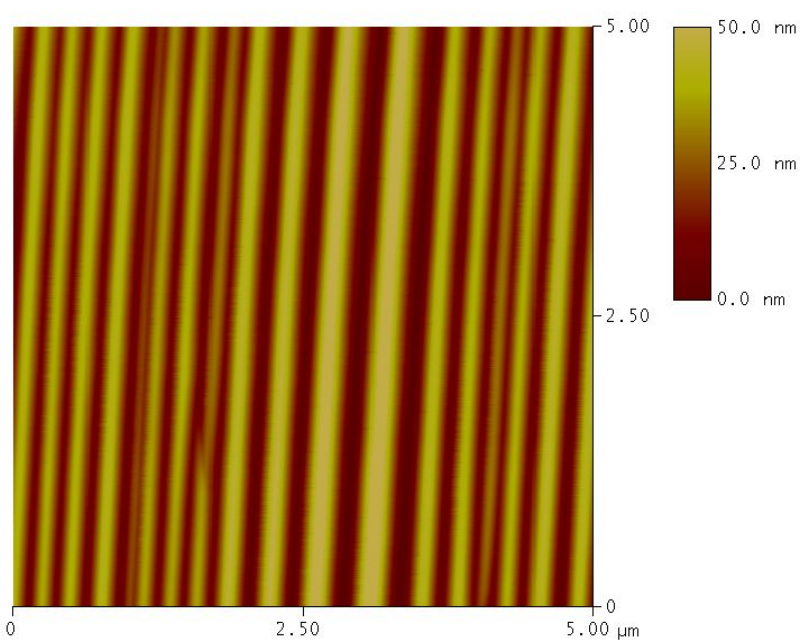

Abbildung 24: AFM-Höhendarstellungen, Bidbereiche jeweils $5 \mu \mathrm{m}^{2}, z$-Skala: $50 \mathrm{~nm}$ (bei $1300^{\circ} \mathrm{C} z$-Skala: 20nm): Mit zunehmender Temperatur steigt die Facettierungsgüte, -höhe und Facettenbreite. 


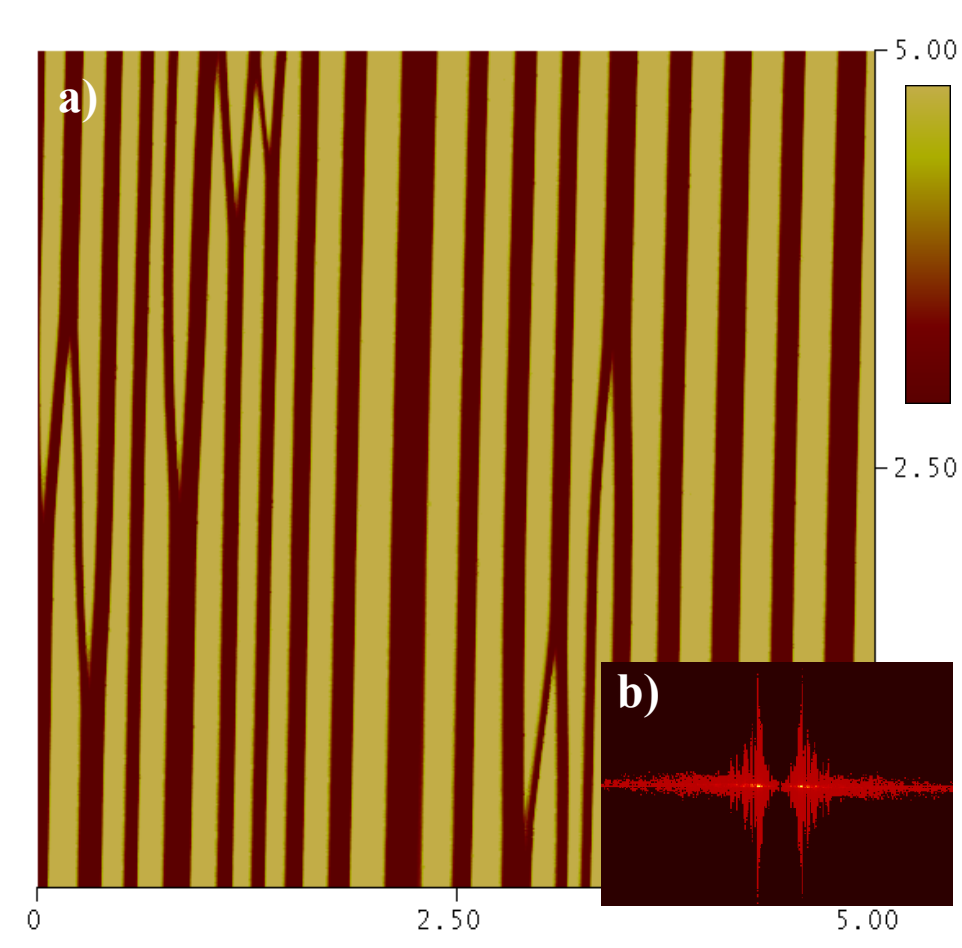

$\mu \mathrm{m}$

Abbildung 25: a) AFM-Amplitudensignal, Bildbereich $5 \mu m^{2}$, $z$-Skala $=0,5 \mathrm{~V}$, Temperung: $24 \mathrm{~h}$ bei $1550^{\circ} \mathrm{C}$, vollständig facettierte Oberfläche mit Defekten an Kontaktlinien von Facettierungsdomänen.

b) 2D-Fourier-Spektrum mit scharfem Maximum bei $x=321 \mathrm{~nm}$, entsprechend der mittleren Periodizität.

Facettierungsdefekte, wie sie Abbildung 25 zeigt, können bei hinreichend langen Temperzeiten partiell ausheilen. Die beobachteten Veränderungen ähneln der Versetzungsbewegung im Festkörper. Zwei Teilfacetten können durch eine Bewegung senkrecht zur Facettierungsrichtung zusammenwachsen und eine durchgehende Facette bilden. Abhängig von der lokalen Umgebung kann eine Teilfacette sich in Facettierungsrichtung verlängern oder verkürzen. Beide Prozesse führen zu einer höheren Facettenordnung und $\mathrm{zu}$ einer effektiven Verbreiterung der Facetten. In der Amplitudensignaldarstellung (Abbildung 25a) einer vollständig facettierten Oberfläche nach Temperung bei $1550^{\circ} \mathrm{C}$ ist unten rechts das 2D-Fourier-Spektrum eingefügt (b). Ein scharfes Maximum bestätigt die hohe Güte der Facettierung und zeigt deren Periodizität von $321 \mathrm{~nm}$.

Für das Facettenwachstum wurden verschiedene Modelle vorgeschlagen, die meisten beruhen auf einem Vorschlag von Mullins [Mul 61]. Gemäß seiner Untersuchungen wächst die Facettenbreite $L$ mit einem Potenzgesetz.

$$
L(t) \propto t^{n}
$$

Dabei ist $t$ die Zeit des Facettenwachstums. Der Exponent $n$ des Potenzgesetzes hängt vom zu Grunde liegenden Massetransportmechanismus ab. Für ein Wachstum aus der Gasphase wurde $n=1 / 2$ berechnet, für Oberflächendiffusion $n=1 / 4$. Experimentelle Arbeiten konnten an verschiedenen Systemen zeigen, dass die Wachstumsgeschwindigkeit jedoch geringer als berechnet ist (z.B. [Pha 91]). Neuere Modelle von Liu et al. [Liu 93] erwarten für zweidimensionale Systeme für das Wachstum einen Exponenten zwischen $n=0,23$ und $n=0,13$, für quasi eindimensionale Systeme ein logarithmisches Wachstum der Facettenbreite mit der Zeit.

$$
L(t) \propto \ln t
$$

Abbildung 26 zeigt die in dieser Arbeit ermittelten Facettenbreiten bei einer Auslagerung von 24h bei $1550^{\circ} \mathrm{C}$. Zum Vergleich sind zwei Datensätze aus der Literatur zur Facettierungsbreite in Abhängigkeit von der Temperzeit eingetragen. Die Auslagerungstemperaturen betrugen dabei $1400^{\circ} \mathrm{C}$ [Wes 04] und $1500^{\circ} \mathrm{C}$ [Hef 97]. Man erkennt, dass die Facettenbreiten im Rahmen der Messgenauigkeit nur wenig von der exakten Auslagerungstemperatur abhängen. Wie schon Heffelfinger et al. vermuteten, ist es aufgrund der relativ großen Verteilungsbreite der Facettenbreite (entsprechend der Fehlerbalken in Abbildung 26 ) nicht möglich, zwischen einem logarithmischen Wachstum oder dem gemäß eines Potenzgesetzes mit kleinem Exponenten $(n=0,13-0,25)$ zu unterscheiden. Somit kann so nicht entschieden werden, ob die Behandlung der Facettierung der $(10 \overline{1} 0)$-Oberfläche von $\alpha-\mathrm{Al}_{2} \mathrm{O}_{3}$ als quasi eindimensionales Problem gerechtfertigt ist. 
Ein Mechanismus, der zur Facettenverbreiterung führt, ist die Bewegung von „Stapelfehlern“, die beim Zusammenwachsen der Facettendomänen zwangsläufig entstehen. Eine eingeschobene Halbfacette kann, vergleichbar mit einem Versetzungsklettern, entlang der Facettierungsrichtung wandern und damit, je nach Bewegungsrichtung, die makroskopische Facettierungsperiode erhöhen oder reduzieren. Treffen zwei Halbfacetten unterschiedlicher Bewegungsrichtung aufeinander, kommt es zu einem Zusammenschluss und damit $\mathrm{zu}$ einer neuen Facette. Bewegen sie sich voneinander weg, so kommt es zu einer Vergrößerung der Facettierungs-

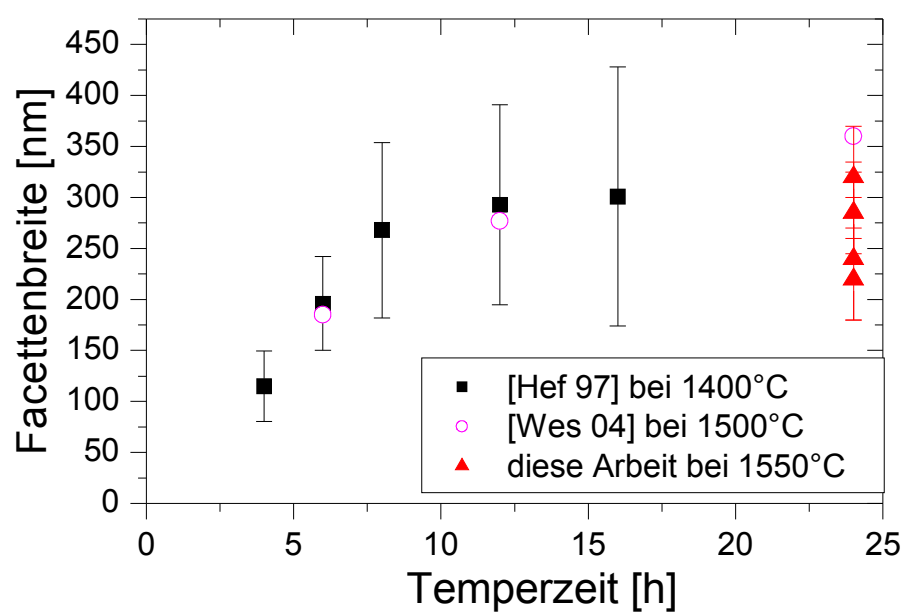

Abbildung 26: Wachstum der Facettenbreite mit der Temperzeit im Vergleich mit Daten von Heffelfinger et al. [Hef 97] und Westphalen et al. [Wes 04]. periode und damit $\mathrm{zu}$ einer Verbreiterung der effektiven Facettenbreite.

Der Abbau der in den Facettenkämmen (und -tälern) gebundenen Energie und der daraus resultierenden elastischen Verspannungsenergie ist die treibende Kraft für die Vergröberung. Diese Energiebeiträge sind deutlich kleiner als die des Gewinns an Oberflächenenergie durch Bildung der günstigen Kristallflächen. Daher ist eine weitgehende kinetische Entkopplung des Wachstums und der Vergröberung der Facettierung zu verstehen. Ein geringer Energiegewinn durch den Abbau von Facetten bei gleichzeitiger Erhöhung der Facettierungsperiode durch Transport senkrecht zur Facettierungsrichtung erfordert lange Temperzeiten.

Die Facettierung der (10 $\overline{1} 0)$-Oberfläche lässt sich somit in folgender Kurzdarstellung zusammenfassen (Abbildung 27).

a) Statistische Bildung von Oberflächenstörungen. Diese fungieren als Nukleationszentren für die ersten Facetten.

b) Wachstum der Facetten in Facettierungsrichtung [1 $1 \overline{2} 10]$ durch schnelle Diffusion entlang der Kanten.

c) Bildung von Facettendomänen durch Nukleation neuer Facetten an den Stellen stärkster Oberflächendeformation, also mittig an den Flanken bestehender Facetten. Gleichzeitig „Aufstauen“" kleiner Facetten zur stabilen Facettenbreite.

d) Zusammentreffen der Facettendomänen

e) Ausheilen von beim Zusammenschluss der Facettierungsdomänen entstanden Defekten.

Eine vollständig facettierte (1010) -Oberfläche besteht aus weitgehend symmetrischen Facetten, die mit der Ausgangsfläche einen Winkel von 17,6 $6^{\circ}$ bilden. Während sich eine sehr glatte Seite

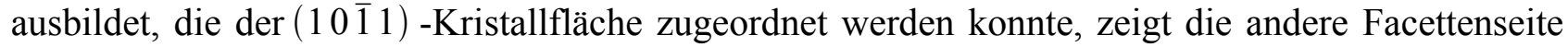
eine Vielzahl von Stufen, die insbesondere an den Ansätzen die Fläche geringster Oberflächenenergie, die $(10 \overline{1} \overline{2})$-Fläche beinhalten. 
a)

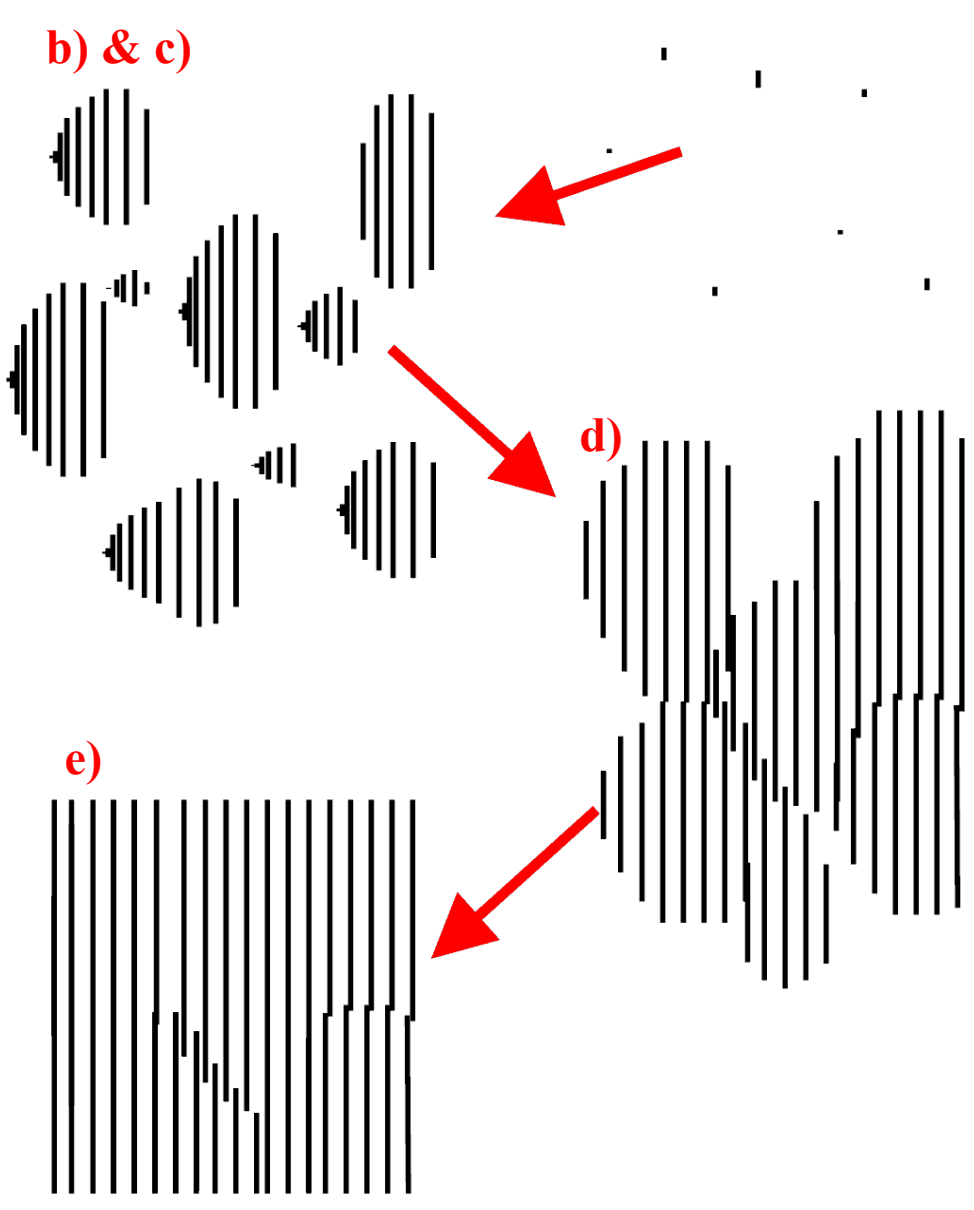

Abbildung 27: Schematische Darstellung der Stadien der Facettierung.
Ein Heizzyklus mit Heizrampen von $400 \mathrm{Grad} / \mathrm{Stunde}$ auf $1550^{\circ} \mathrm{C}, 24 \mathrm{stün}-$ diger Temperung an Luft gefolgt von eine Abkühlrampe von ebenfalls 400Grad/Stunde auf Raumtemperatur hat sich als geeignet erwiesen vollständig facettierte Oberflächen herzustellen, bei denen die Defektdichte in der Facettierung durch Ausheilprozesse bereits deutlich gesenkt werden konnte und eine konstante Facettenbreite mit einer mittleren Facettierungsperiode von $320 \mathrm{~nm}$ vorliegt.

Die optimale Facettierungsperiode $D_{o p t}$ wird maßgeblich durch die Materialeigenschaften des $\mathrm{Al}_{2} \mathrm{O}_{3}$ (Elastizitätsmodul $E$ und Gitterkonstante $a$ ) sowie durch weitere Spannungsbeiträge bestimmt. Die Selbstenergie der Facettenlinien $\eta$ (Facettenkämme bzw. Täler) bietet aber einen möglichen Ansatzpunkt die Periodenlänge $\mathrm{zu}$ beeinflussen. Bringt man vor der Temperung eine Deckschicht auf das Substrat auf, so wird die Grenzflächenenergie zwischen Schicht und Substrat Einfluss auf $\eta$ nehmen und die elastischen Eigenschaften der Oberfläche verändern. Daher bietet die Aufbringung von hochschmelzenden, geeigneten Deckschichten in Zukunft einen Ansatz, die Facettierung zu steuern. In wieweit die Unterdrückung des bei niedrigen Temperaturen dominanten Oberflächendiffusionsanteils die Facettenbildung behindert, ist durch Experimente zu klären.

In der Nanofacettierung von Festkörperoberflächen durch Temperungen liegt eine großes Potential, auf einfache Weise großflächig und mit geringem Aufwand sehr regelmäßige Strukturierungen im technisch immer interessanter werdenden Bereich von wenigen Nanometern zu erzielen.

Im Rahmen dieser Arbeit wurde diese Strukturierungsmethode soweit optimiert und durch den Temperprozess gezielt beeinflusst, dass geeignete Substrate mit Periodizitäten von ca. 320nm und Facettenhöhen von ca. 50nm mit hoher Zuverlässigkeit und sehr gut reproduzierbar hergestellt werden konnten. 


\section{Transfer der Periodizität in Felder von Nanodrähten}

\subsection{Nanodrähte durch Schattendeposition}

Durch Deposition von Material unter einem flachen Winkel bezüglich der makroskopischen Substratoberfläche (Abbildung 28) kann die besondere Oberflächenmorphologie der facettierten (10 10$)$-Saphiroberfläche genutzt werden, um gezielt Nanodrähte herzustellen. Dabei bewirkt bei hinreichend flachem Winkel der Selbstabschattungseffekt der Facetten, dass nur die dem Materialstrom zugewandte Facettenseite mit dem Depositionsmaterial belegt wird, während die abgewandte Seite durch die Facette selbst abgeschattet wird. Nanodrähte zeichnen sich durch besondere Aspektverhältnisse (Länge zu Breite) aus, was sich in Form einer Anisotropie der Eigenschaften widerspiegeln sollte. Das Nukleations- und Wachstumsverhalten von verschiedenen Metallen (Eisen, Kobalt und Gold) auf der facettierten Unterlage wird vorgestellt und das elektrische sowie magnetische Verhalten des entstandenen Drahtfelds analysiert.

Die Materialbedeckung wird im Wesentlichen durch zwei Einflussfaktoren bestimmt. Einerseits hat das Depositionsverfahren einen Einfluss auf den Bereich, in dem Material auftrifft (siehe auch Abbildung 29), andererseits ist der Wachstumsmodus und im Zusammenhang damit die Diffusionslänge des deponierten Materials auf der Oberfläche entscheidend.

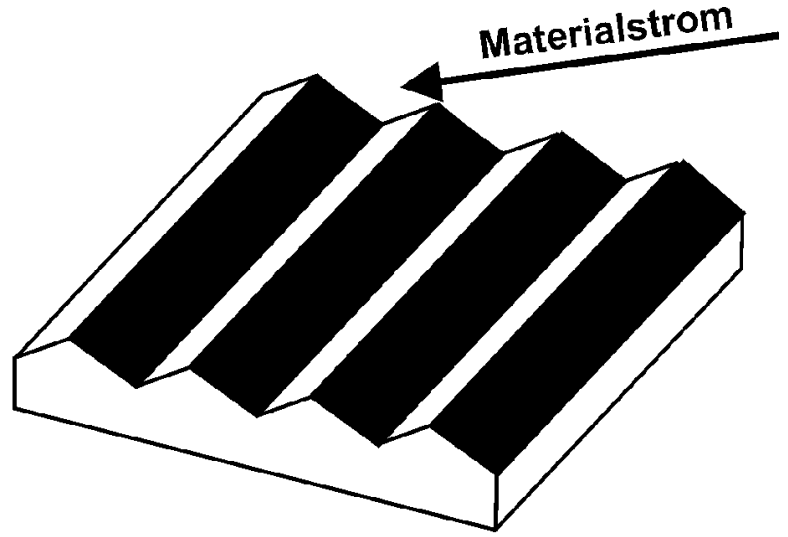

Abbildung 28: Abschattungseffekt durch flache Deposition.

\subsubsection{Einfluss des Depositionsverfahrens}

Die Energie der auf die Oberfläche auftreffenden Atome und die Divergenz des Materialstrahls haben bei einer Deposition, die geometrische Abschattungseffekte ausnutzt wesentlichen Einfluss auf die erzielbare Gestalt.

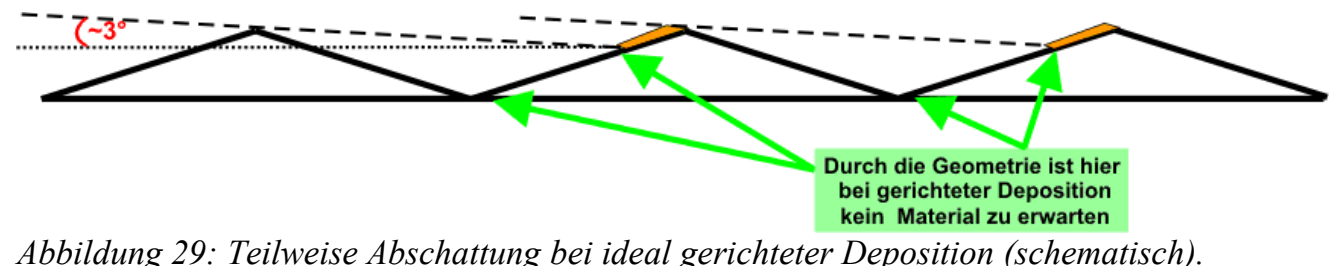

Bei der gerichteten Deposition mittels Elektronenstrahldeposition auf die (100)- resp. (010)-Seiten von hydrothermisch geätzten Kanälen eines $\mathrm{NaCl}(110)$ Substrats ist ein Depositionswinkel von $25^{\circ}$ ausreichend, um eine Deposition nur auf der dem Materialstrom zugewandten Seite zu erreichen 
[Sug 97]. Bei kleineren Winkeln finden Sugawara et al. eine deutliche Abschattung der dem Material zugewandten Seiten im Bereich der Täler (siehe Abbildung 29). Auf diese Weise konnten Eisendrähte mit den Dimensionen $45 \mathrm{~nm}$ x $13 \mathrm{~nm} \times 10 \mu \mathrm{m}$, sowie heterogen in Golddrähten eingebettete Kobaltteilchen [Sug 01] hergestellt werden.

Dahingegen zeigen die Untersuchungen im Rahmen dieser Arbeit, dass bei flacheren Facetten $\left(17,6^{\circ}\right)$ und dem weniger stark gerichteten Verfahren des Magnetron-Sputterns erst eine Deposition unter deutlich flacheren Winkeln $\left(3^{\circ}-5^{\circ}\right)$ zu den gewünschten separierten Drähten führt. Dahermuss mit einer zusätzlichen Bedeckung abgeschatteter Bereiche gerechnet werden. Um diesen Effekt zu minimieren, wurde in dieser Arbeit ein Depositionswinkel von $3 \pm 1^{\circ}$ verwendet, was mit einer deutlichen Reduktion der Aufwachsrate auf der zu bedeckenden Seite einhergeht.

Die Morphologie der entstehenden drahtartigen Strukturen ist dabei wesentlich vom Aufwachsverhalten des gewählten Materials auf dem jeweiligen Substrat abhängig, wie die im folgenden beschriebenen Untersuchungen zur Deposition verschiedener Metalle (Eisen, Gold und Kobalt) auf facettiertem m-plane $\mathrm{Al}_{2} \mathrm{O}_{3}$ zeigen.

\subsubsection{Inselwachstum und das Überwachsen der Facettenkämme am Beispielsystem Gold}

Gold zeigt bei der Deposition mittels Magnetron-Sputtern ausgeprägtes Inselwachstum und eine hohe Mobilität auf den Facettenseiten und über die Facettenkämme hinweg.

Daraus resultiert eine Morphologie, wie sie beispielhaft in der rasterkraftmikroskopischen Abbildung 30 dargestellt ist. Die Dicke des deponierten Materials lässt sich aus den Aufwachsraten auf $<20 \mathrm{~nm}$ abschätzen. Die Darstellung verdeutlicht, dass auf dem Materialstrom zugewandten Facettenseite das Gold in einer großen Anzahl von Körnern mit einem mittleren Durchmesser von 35-50nm aufwächst. Dies entspricht dem mittleren Durchmesser von Wachstumssäulen in Schichtsystemen der Komponenten Eisen und Gold. Die Körner sind durch den flachen Depositionswinkel von $3 \pm 1^{\circ}$ leicht in Richtung des Materialstroms gestreckt. Die

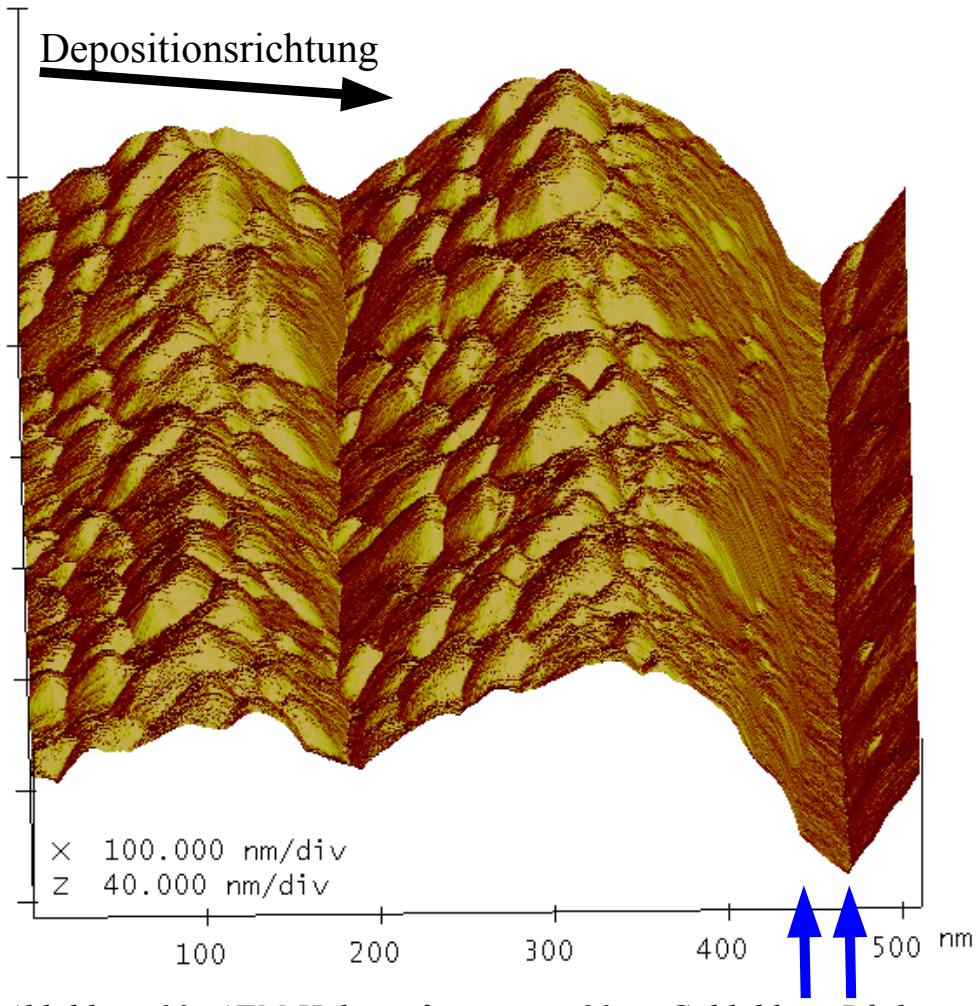

Abbildung 30: AFM-Höheninformaton:<20nm Gold, blaue Pfeile markieren einen Gold-freien Bereich im Facettental. Facettenkämme werden, ohne dass sie noch als ausgezeichnete Punkte erkennbar wären, überwachsen. Gold bedeckt bei den meisten Facetten auch die dem Materialstrom abgewandten Seiten vollständig. Lediglich bei besonders hohen Facetten finden sich am Facettenansatz in den Tälern Substratbereiche, die frei von 
deponiertem Gold sind. In Abbildung 30 ist ein solcher Streifen durch blaue Pfeile markiert.

Abbildung 31 zeigt ein Schnittbild zur Höheninformation senkrecht zur Facettierungsrichtung. Die unterbrochene Linie deutet den Verlauf der Facettenfläche an. Der überwiegende Anteil des Golds findet sich auf den linken, dem Materialstrom zugewandten Facettenseiten. Die Abschattung führt $\mathrm{zu}$ einer verringerten Materialmenge im unteren Bereich der Facetten, wodurch im Querschnitt ein

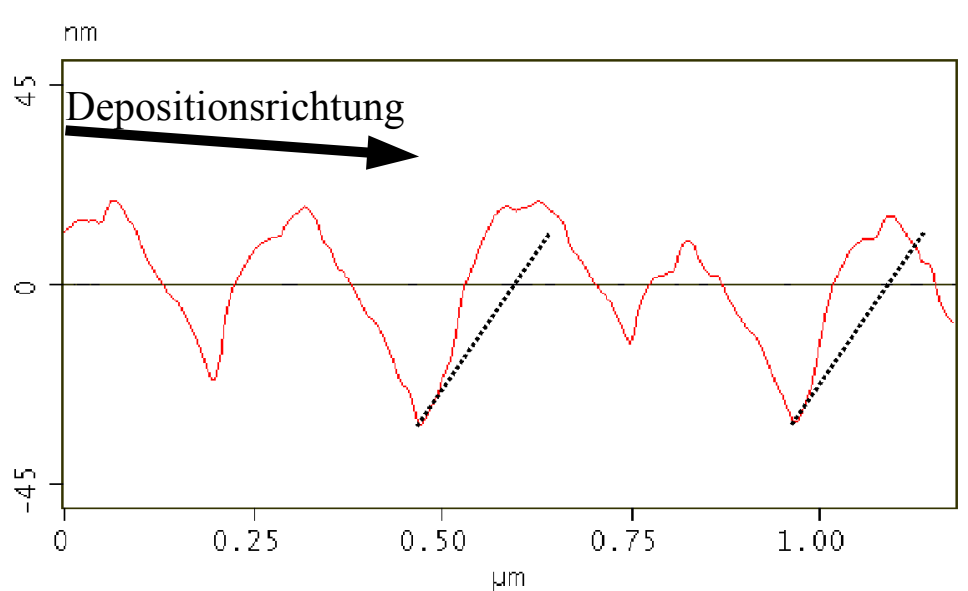

Abbildung 31: AFM-Höheninformation: Schnittbild senkrecht zur Facettierungsrichtung. „bauchiger“ Verlauf der Oberfläche entsteht.

Die starke Neigung von Gold zu Inselwachstum, vor allem aber die hohe Mobilität der Goldatome auch über die Facettenkämme hinweg bei einer Substrattemperatur von nur 293K, verhindert also sogar unter sehr flachem Winkel, isolierte Gold-Nanodrähte durch Schattendepositon zu erzeugen. Für die Oberflächendiffusion von Silber auf $\mathrm{Al}_{2} \mathrm{O}_{3}$ geben Beke et al. für $\mathrm{T} / \mathrm{T}_{\mathrm{m}} \approx 0,4$ für $\mathrm{D}_{0}=8,1 \cdot 10^{-7} \mathrm{~m}^{2} / \mathrm{s}$ und eine Aktivierungsenergie von $192 \mathrm{~kJ} / \mathrm{mol}$ an. Für Gold sind vergleichbar hohe Werte zu erwarten [Bek 95].

\subsubsection{Wachstum von Nanodrähten auf facettiertem $\mathrm{Al}_{2} \mathrm{O}_{3}$}

Die Deposition von Gold hat gezeigt, dass bei der Präparation von Drähten durch Schattendeposition die Oberflächendiffusion dafür entscheidend ist, wieviel Material auf die Schattenseite gelangt. Wünschenswert wäre ein Lagenwachstum ohne ein Überwachsen der Facettenkämme. Da aber nicht nur morphologische Eigenschaften der deponierten Strukturen von Interesse sind, sondern auch die magnetische Wechselwirkung von Nanodrähten und -partikeln von großer Bedeutung sind, standen bei der Auswahl weiterer Materialien für die Abscheidung von Drähten ferromagnetisch ordnende Materialien im Vordergrund.

Unter einem Einfallswinkel von $3 \pm 1^{\circ}$ zur Substratoberfläche konnten erfolgreich Eisen-Nanodrähte hergestellt werden, während die Deposition bei größeren Winkeln zu einer maßgeblichen Bedeckung beider Facettenseiten führte. Abbildung 32 zeigt die durch Abschattung separierten Drähte in einer rasterelektronenmikroskopischen Abbildung.

Dabei entspricht ein heller Kontrast einer Bedeckung mit Eisen. Die mittlere Breite der Eisendrähte (unter Berücksichtigung des Facettierungswinkels von $17,6^{\circ}$ ) beträgt $135 \pm 13 \mathrm{~nm}$, der mittlere Abstand $87 \pm 15 \mathrm{~nm}$ entsprechend einer verringerten Facettierungsperiode im ausgewerteten Bereich. 


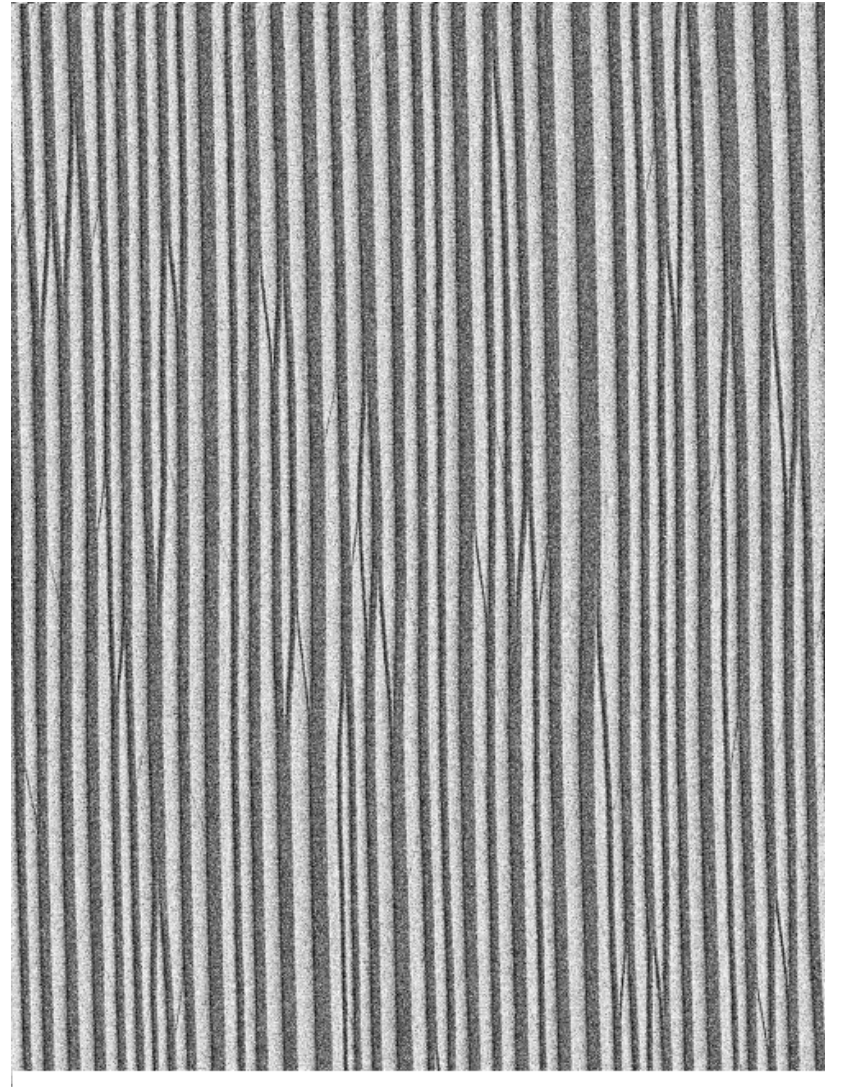

$1 \mu \mathrm{m}$
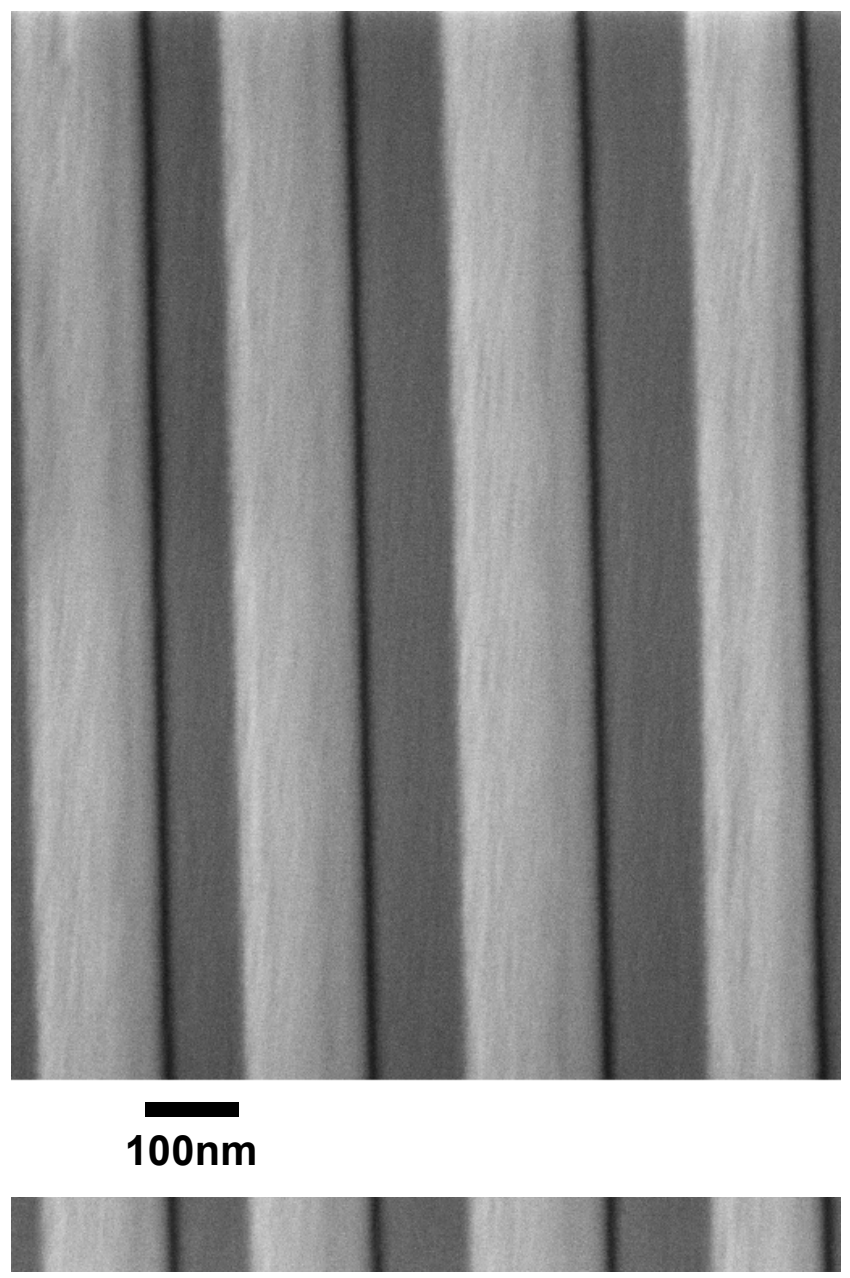

Abbildung 32: SEM-Aufsicht: Eisendrähte (hell) durch Deposition unter flachem Winkel ( $\left.3^{\circ}\right)$.

Neben den Konstrastunterschieden durch die stark unterschiedliche elektrische Leitfähigkeit der Eisendrähte und des Substrats im Rasterelektronenmikroskop bietet die Rasterkraftmikroskopie (AFM) gute Voraussetzungen, die Bedeckung der verschiedenen Facettenseiten zu untersuchen. Mittels AFM wurde im TappingMode eine Detailaufnahme einer einzelnen Facette erstellt. Abbildung 33 zeigt diese in einer dreidimensionalen Darstellung. Deutlich erkennbar ist die unterschiedliche Oberflächenbeschaffenheit auf den verschiedenen Facettenseiten. Während die linke Seite sehr kontrastreich und rau abgebildet wird, ist die rechte Facettenseite sehr glatt. Die Streifen regelmäßigen Kontrastwechsels, die ebenfalls auf der rechten Seite besonders deutlich sind, sind als Messartefakt zu interpretieren und geben keine Probeneigenschaft wieder. Die Schnittdarstellung (Abbildung 34) bestätigt die unterschiedliche Beschaffenheit der Facettenseiten. Auf der linken Facettenseite ist eine gleichmäßige Bedeckung von ca. 10-12nm erkennbar. Die unkorrelierten Rauigkeiten auf dieser Seite sind mit 2,3nm RMS über einen Faktor 5 größer als auf der glatten Substratseite $(0,42 \mathrm{~nm}$ RMS). Abbildung 34 bestätigt wiederum die aufgrund der TEMQuerschnittspräparationen getroffene Aussage, dass die Facetten weitgehend symmetrische Facettenseiten bzgl. Breite und Winkel zur vormaligen Substratoberfläche aufweisen.

Auch wenn die dem Materialstrom abgewendete Facettenseite sehr glatt erscheint, kann daraus nicht zwangsläufig geschlossen werden, dass diese unbedeckt ist. Neben der Höheninformation ist es insbesondere für die Untersuchungen zur Bedeckung sinnvoll, die Verschiebung in der Phase der Cantileverschwingung gegenüber der Anregung darzustellen. Die Messung erfolgte im TappingMode mit einer nicht-magnetischen Spitze. Durch die Sensitivität der Phasenverschiebung für tribologische Eigenschaften ist eine Unterscheidung von bedecktem und unbedeckten Substrat 


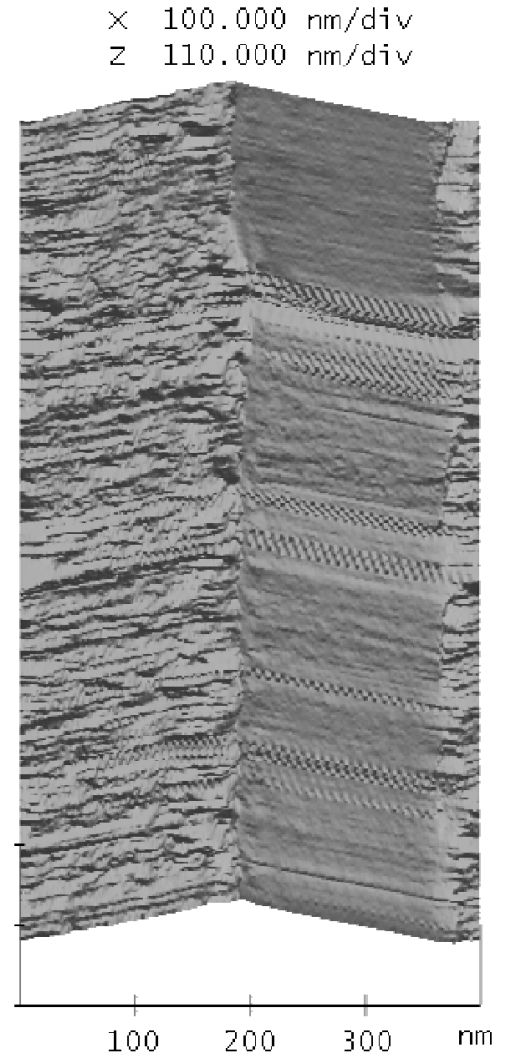

Abbildung 33: AFM- Höhendarstellung: Unterschiedliche Oberflächenbeschaffenheit einer einzelnen Facette, linke Facettenseite mit Eisen bedeckt.

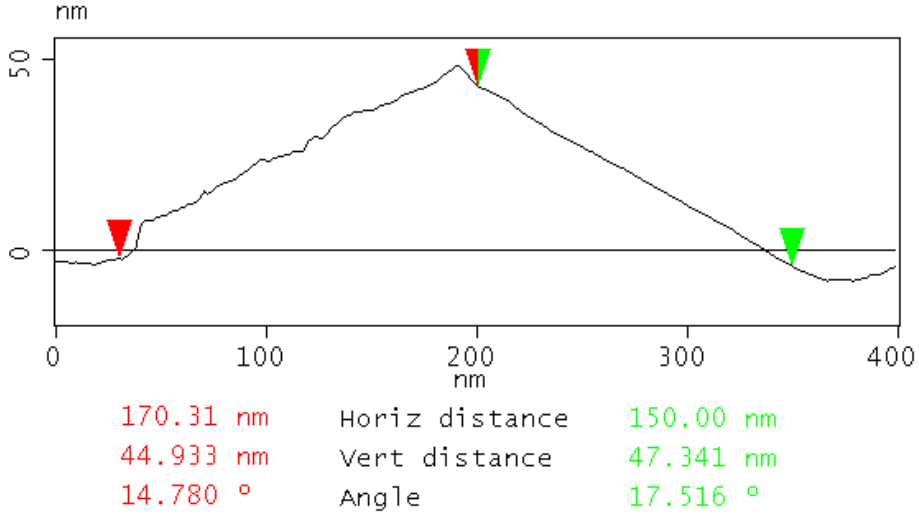

Abbildung 34: Schnittdarstellung zur Facette aus Abbildung 33.

möglich. Ein Vergleich der Höhen- bzw. Phasendarstellung der simultan aufgezeichneten Daten eines $2 \mu \mathrm{m}^{2}$ Probenbereichs in Abbildung 35 zeigt deutliche Unterschiede für beide Facettenseiten. Der Kontrast im Phasensignal drückt hier in erster Linie die große Differenz in den Härten aus. Während Saphir eine Härte (Mohs) von 9 hat, ist diese bei Eisen mit 4,5 gerade halb so groß. Durch den TappingMode wird nur eine geringe Kraft auf die Probe ausgeübt, so dass auch bei sehr geringen Bedeckungen noch eine gute Unterscheidbarkeit von Substrat und Substrat mit dünner Schicht gegeben ist.

Abbildung 36 zeigt ortsrichtige Zusammenschnitte aus Höhen- und Phasenabbildung. Unterhalb der schwarzen Trennlinie ist die Phasenverschiebung, oberhalb die Höheninformation perspektivisch

a)

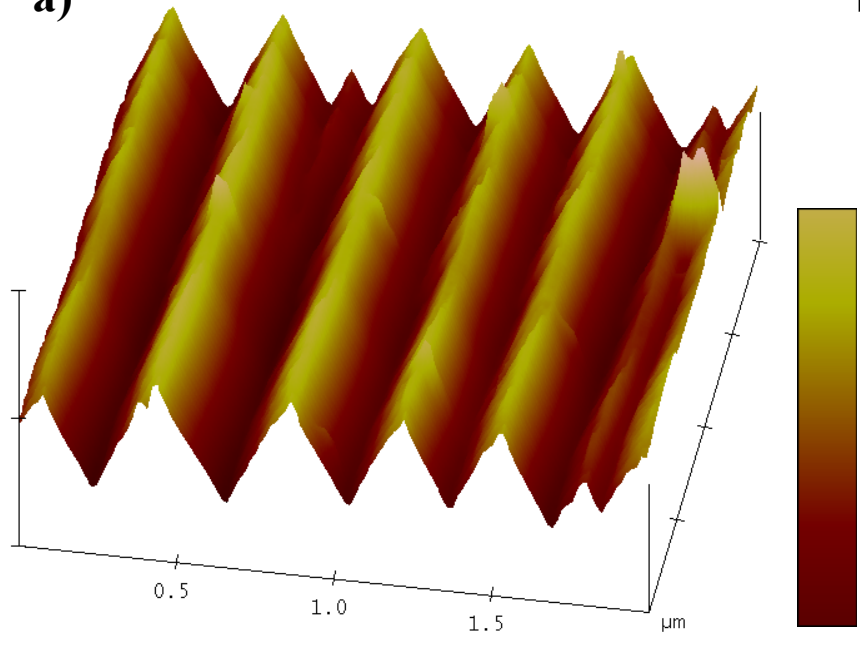

b)

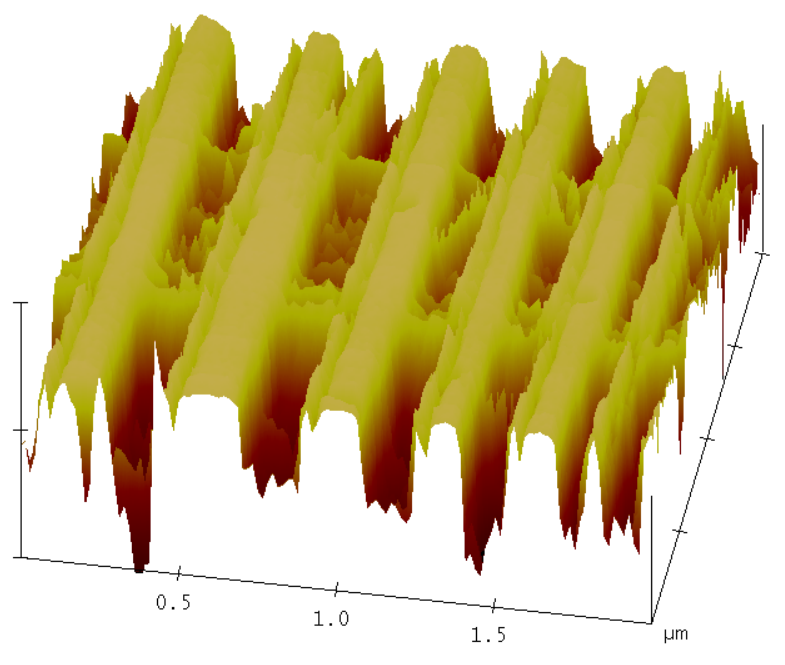

Abbildung 35: AFM mit nicht-magnetischer Spitze: ca. 10nm dicke Eisendrähte a) Höhenabbildung, z-Skala: $75 \mathrm{~nm}$ und b) Phasenabbildung, z-Skala: $15^{\circ}$. 
dargestellt. Abbildung 36a) zeigt, dass auf den von links unten nach rechts oben ansteigenden Facettenseiten ein heller Phasenkontrast gemessen wird. Eine Drehung der Probe um $180^{\circ}$ (Abbildung 36b) hat eine Umkehr des Phasenkontrasts zur Folge, d.h. die linke Facettenseite zeigt nun einen dunklen Phasenkontrast. Dies bestätigt die Beobachtung, dass hauptsächlich die der Quelle zugewandte Seite mit Eisen beschichtet wird. Vergleicht man die Facettenkammlage in der Höhendarstellung mit der Position des Kontrastwechsels in der Phasendarstellung, so erkennt man, dass die dem Materialstrom abgewandte Facettenseite nur bis $\mathrm{zu}$ einer Breite von ca. 40nm mit Eisen belegt ist.

a)

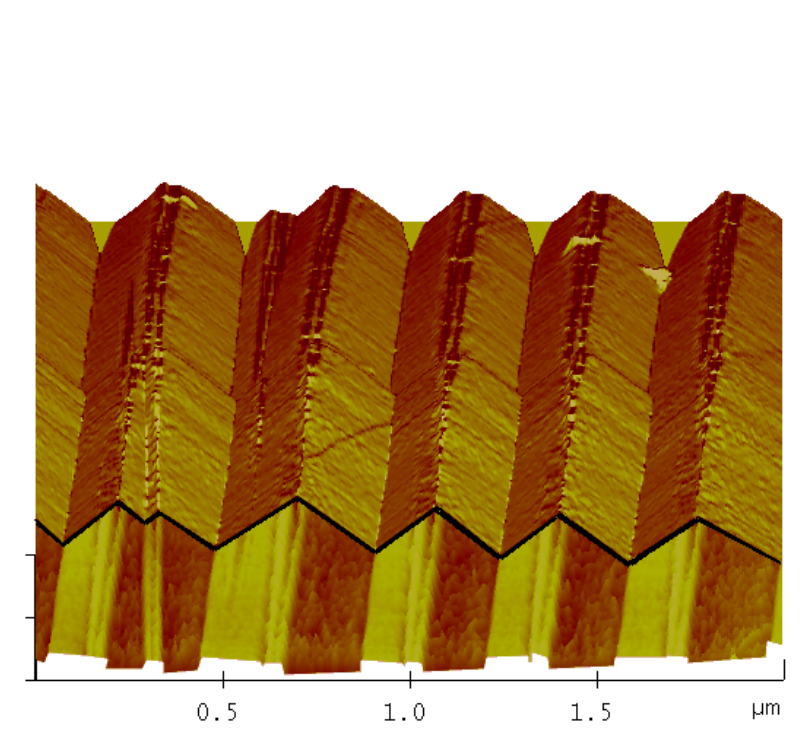

$0^{\circ}$

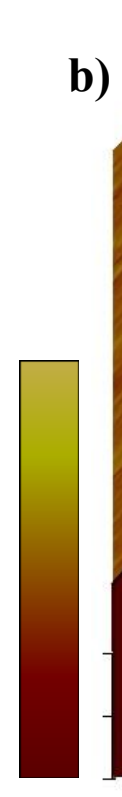

b)

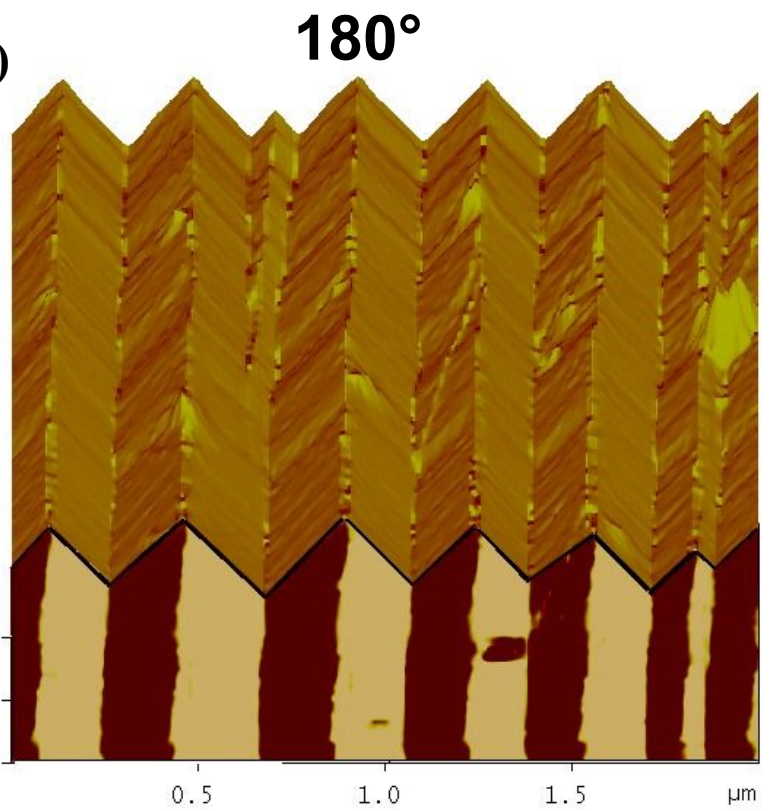

Abbildung 36: AFM mit nicht-magnetischer Spitze: ca. 10nm dicke Eisendrähte. Ortsrichtiger Zusammenschnitt aus Höhen- (oben) und Phasenbild (unten) an der schwarzen Linie, z-Skala: $75 \mathrm{~nm}$ bzw. $7^{\circ}$, a) $0^{\circ}$ und b) nach Drehung der Probe um $180^{\circ}$.

Das Überwachsen der Facettenkämme zeigt auch ein Vergleich von Höhen- und Amplitudenbild (Abbildungen 37 und 38). Das Amplitudenbild zeigt das Gefüge der Eisenschicht besonders plastisch, während beim Höhenbild der starke Topographiekontrast durch die Facetten überwiegt. Mit Eisen bedeckte Bereiche sind im Amplitudenbild durch hellen Kontrast sichtbar. Auf den Kämmen der Facetten haben sich elliptische Körner gebildet (Halbachsen $22 \pm 2 \mathrm{~nm}$ bzw.14 $\pm 2 \mathrm{~nm}$ ), deren längere Halbachsen senkrecht zur Facettierungsrichtung bzw. in Richtung des Materialstroms liegen. Wie eine Veränderung der Abtastrichtung zeigt, ist diese Kornform kein durch die Spitzengeometrie bedingtes Artefakt. 

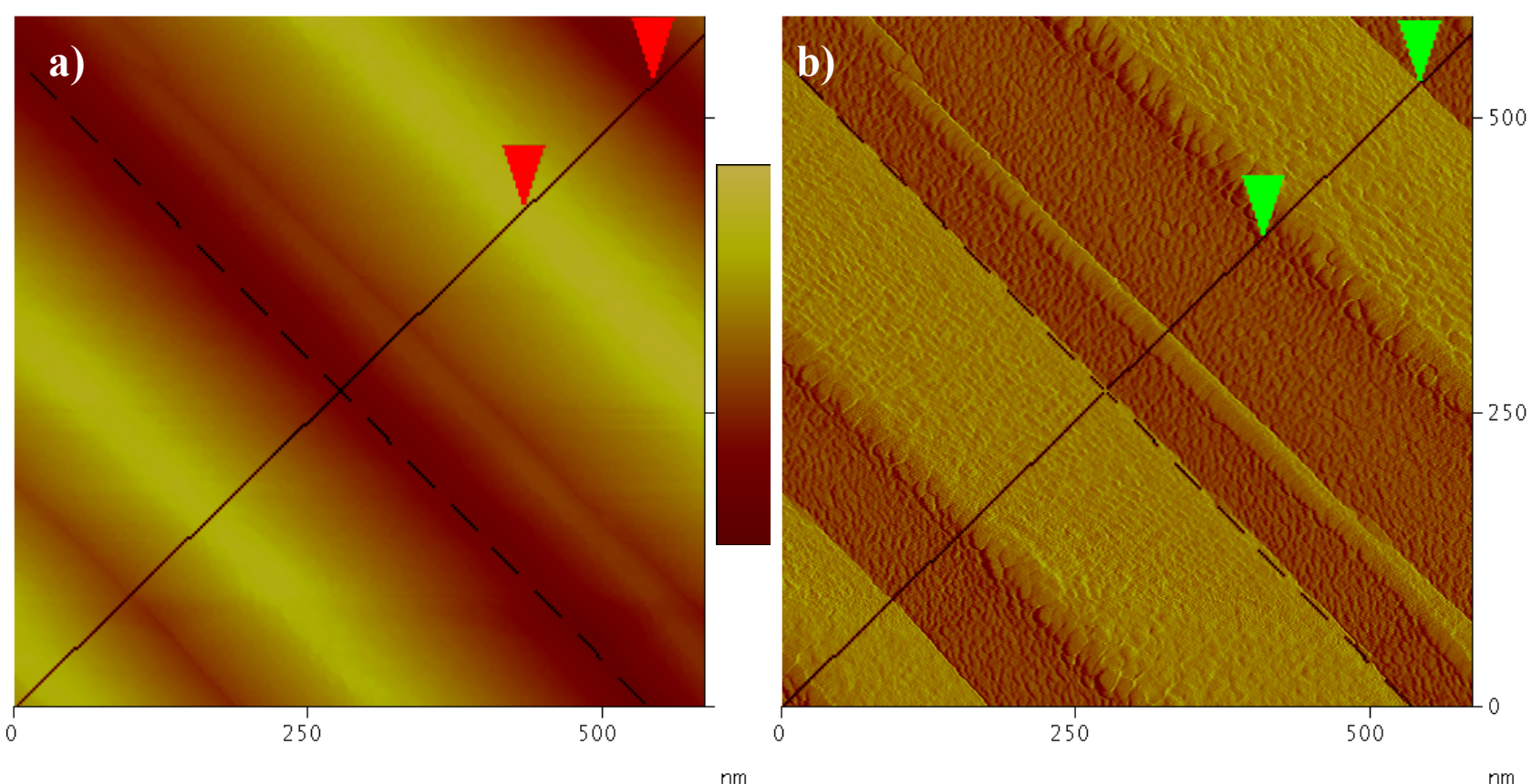

Abbildung 37: a) AFM-Höhendarstellung und b) Amplitudenabbildung, ca. 10nm dicke Eisendrähte, z-Skala: 100nm bzw.0,3V. Das Korngefüge des Eisens (heller Kontrast) ist im Amplitudenbild aufgelöst. Die Depositionsrichtung ist von rechts oben nach links unten.

Abbildung 38 zeigt nun eine Querschnittsdarstellung entlang der in Abbildung 37 eingezeichneten Linie. Überlagert ist der Schnitt in Höhen- (rot) und Amplitudenbild (grün) dargestellt. Durch Pfeile markiert sind im Höhenbild Spitze und Tal der Facettenseite, auf der die Eisenschicht aufgebracht wurde. Auch in der Schnittdarstellung ist ein leichtes Überwachsen der Facettenspitze durch eine Überhöhung erkennbar. Typische Werte für Höhe $(\sim 58 \mathrm{~nm})$ und Breite $(\sim 155 \mathrm{~nm})$ der Facettierung können ermittelt werden. Der Bereich, der im Amplitudenbild (Abbildung 37a) als Eisendraht identifiziert werden kann, ist in Abbildung 38 mit grünen Markern sowie unterbrochenen vertikalen Linien gekennzeichnet. Die Positionen stimmen gut mit dem Tal bzw. mit der Grenze der Überhöhung des Höhenschnitts überein und es ergibt sich eine Breite dieses Drahtes von 186nm.

Das Überwachsen der Facettenkämme erklärt, warum mittels Rasterelektronenmikroskopie trotz symmetrischer Facetten eine gegenüber der Drahtbreite kleinere Separationsbreite der Drähte gemessen wurde.

$\mathrm{Da}$ im SEM der Kontrast im Wesentlichen durch die Leitfähigkeit und nicht durch die Topologie bestimmt ist, können die Breiten der hellen Streifen in Abbildung 32 nicht mit der Facettenseitenbreite, sondern nur mit der Eisenbedeckung direkt korreliert werden.

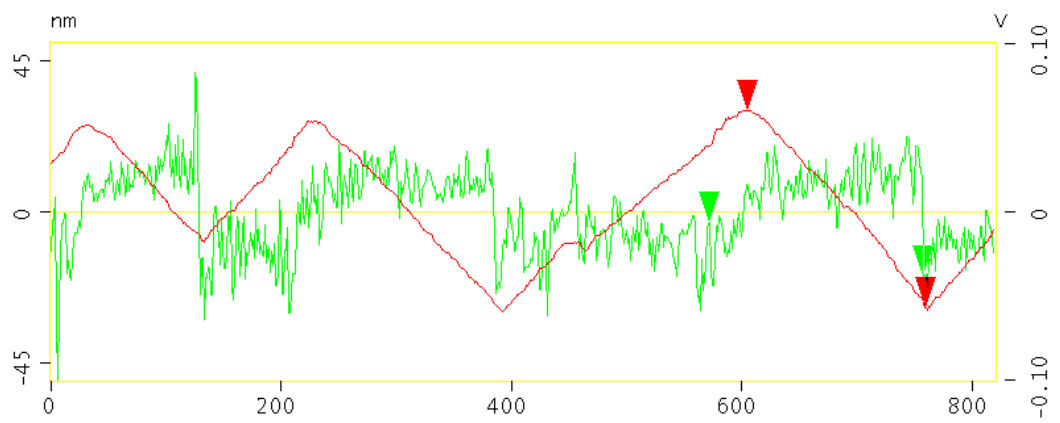

Abbildung 38: AFM-Schnittdarstellung zur Höhen- und Amplitudendarstellung in Abbildung 37. 
Zusammenfassend kann gesagt werden, dass es gelungen ist, mittels flacher Deposition im Wesentlichen die der Quelle zugewandte Seite der Facetten zu bedecken und somit separierte Eisendrähte einer Dicke von etwa 10-12nm und einer Breite von etwa 180nm herzustellen.

\section{In-situ Leitwertmessungen bei der Fe-Drahtabscheidung}

Die Leitfähigkeiten der auf facettierten Substraten deponierten Eisendrähte wurden während der Deposition mittels 4-Punktmessung bestimmt. Es erfolgten simultan Messungen in Facettierungsrichtung und senkrecht dazu. Da aufgrund des sehr flachen Depositionswinkels der stromtragende Querschnitt durch die inhomogene Materialverteilung nicht wohldefiniert ist, werden im Folgenden die Leitwerte und nicht die spezifischen Größen angegeben.

Die anisotrope Entwicklung des Leitwerts in den beiden unterschiedlichen Messrichtungen ist in Abbildung $39 \mathrm{zu}$ sehen. Während der Leitwert in Facettierungsrich-

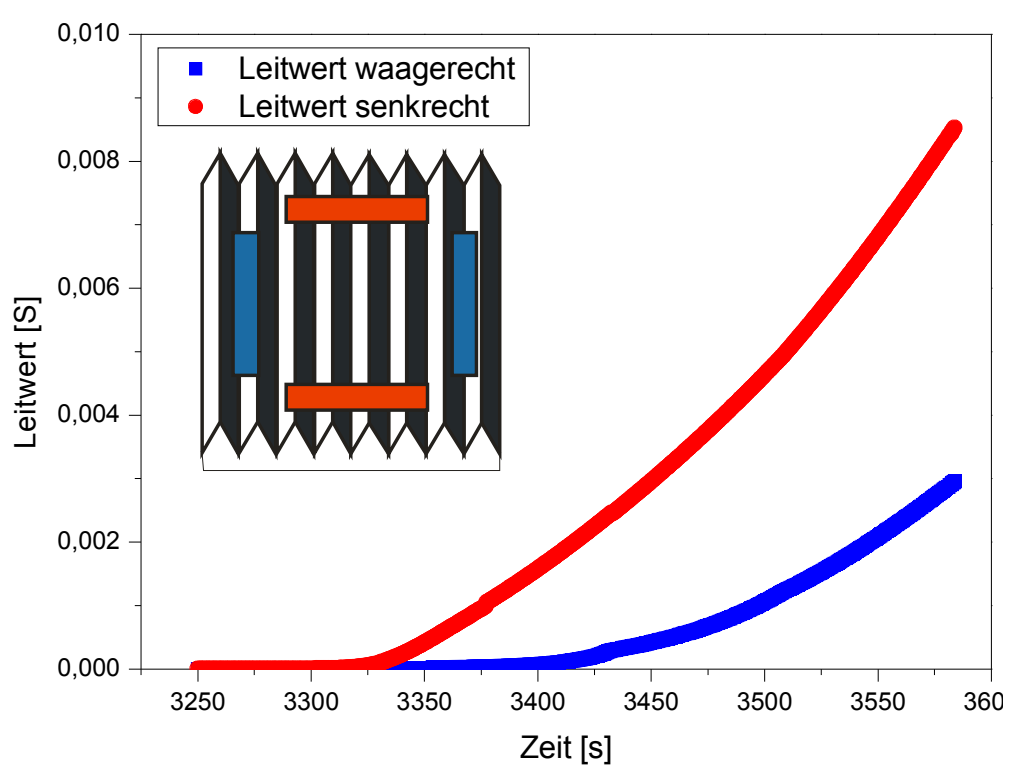

Abbildung 39: Leitwertmessung während der Deposition von EisenNanodrähten auffacettiertem $\mathrm{Al}_{2} \mathrm{O}_{3}$. tung $\mathrm{ab} 40 \mathrm{~s}$ nach Öffnen der Substratblende (entsprechend einer mittleren Schichtdicke von etwa 4,5 $\pm 1,5 \mathrm{~nm}$ ) ansteigt, ist die dreifache Zeit nötig, um einen signifikant von Null verschiedenen Leitwert senkrecht zur Facettierungsrichtung zu erhalten. Die Tatsache, dass auch in dieser Richtung ein Leitwert gemessen wird, bestätigt die Beobachtungen aus den SEM-Untersuchungen, dass auch auf der quellenabgewandten Facettenseite geringe Mengen Eisen deponiert werden. Die Perkolation bei $4,5 \pm 1,5 \mathrm{~nm}$ ist zum einen konsistent mit dem Gefügebild, das sich aus den AFM-Untersuchungen (z.B. Abbildung 37) ergibt. Eisen bildet verglichen mit Gold nur kleine Inseln. Zum anderen kann auch bei Leitfähigkeitsmessungen während der Multilagendeposition bei Schichtdicken $<2 \mathrm{~nm}$ keine Leitfähigkeit gemessen werden. Der Leitwert setzt senkrecht zur Facettierungsrichtung nicht nur später ein, e ist auch generell geringer. Nach Beendigung der Deposition ist der Leitwert in Drahtrichtung um den Faktor 3 größer als senkrecht dazu.

In-situ Leitfähigkeitsmessungen zeigen eine Perkolation für $d>4,5 \pm 1,5 \mathrm{~nm}$, so dass von VolmerWeber-Wachstum [Vol 26] mit entsprechend kleiner Inselgröße auszugehen ist. Die niederenergetische Deposition und die Leitfähigkeit auch senkrecht zur Facettierungsrichtung legen eine geringe Bedeckung der Schattenseite der Facetten nahe.

\section{$\underline{\text { Magnetisierungsverhalten eines Eisen-Nanodrahtfelds }}$}

Die ausgezeichnete Form, insbesondere die extremen Aspektverhältnisse der Nanodrähte, sollten 
auch auf das magnetische Verhalten der Proben einen deutlichen Einfluss haben. Dabei spielt sowohl die Form des einzelnen Drahts (Formanisotropie), als auch die Anordnung der Drähte zueinander (magnetostatische Kopplung) eine Rolle.

Die Anordnung von Eisendrähten auf facettiertem m-plane Saphir zeigt im Zustand direkt nach der Herstellung ein sehr außergewöhnliches Magnetisierungsverhalten. Abbildung 40 verdeutlicht dieses Verhalten anhand von Messungen des MOKE in einem externen Magnetfeld bis zu 850Oe in Abhängigkeit vom Winkel zwischen externer Magnetfeldrichtung und Facettierungsrichtung. Liegt das Feld parallel zur Facettierungsrichtung $\left(0^{\circ}\right.$ bzw. $\left.180^{\circ}\right)$ an, so wird eine rechteckige Hysterese mit $\mathrm{H}_{\mathrm{c}}=383 \pm 5 \mathrm{Oe}$ beobachtet. Die Messungen zeigen ein maximales Verhältnis von Remanenzmagnetisierung $M_{r}$ zur Sättigungsmagnetisierung $M_{s}, M_{r} / M_{s}=1$.

Vergleicht man die gemessenen Magnetisierungskurven (für $0^{\circ}$ bzw. $180^{\circ}$ und $90^{\circ}$, Abbildung 40) mit den idealisierten eines eindomänigen Teilchens (Abbildung 41), so zeigt sich, dass das Feld von Nanodrähten eine Hysterese aufweist, die einer uniaxialen Anisotropie in Facettierungsrichtung entspricht. Die Formanisotropie begünstigt diese, wohingegen mit zunehmendem Winkel zwischen externem Feld und Facettierungsrichtung der Entmagnetisierungsfaktor wächst und das Verhältnis von Remanenz- zu Sättigungsmagnetisierung $M_{r} / M_{s}$ sinkt. Bei einem Winkel von $90^{\circ}$ dreht die Magnetisierung gleichmäßig in Richtung des äußeren Felds. Da diese Magnetisierungsrichtung aber durch die Formanisotropie ungünstig ist, dreht die Magnetisierung bei Absenken des Feldes instantan wieder aus dieser Richtung heraus. Da es sich bei den Proben um sehr dünne Drähte handelt, kann die Verkippung aus der Facettenseitenebene hinaus vernachlässigt werden. Schon bei einem Winkel von $45^{\circ}$ kann durch das zur Verfügung stehende Feld von 8500 e keine vollständige Sättigung mehr erreicht werden. Das Koerzitivfeld ändert sich bis zu einer nahezu senkrechten Ausrichtung von externem Feld
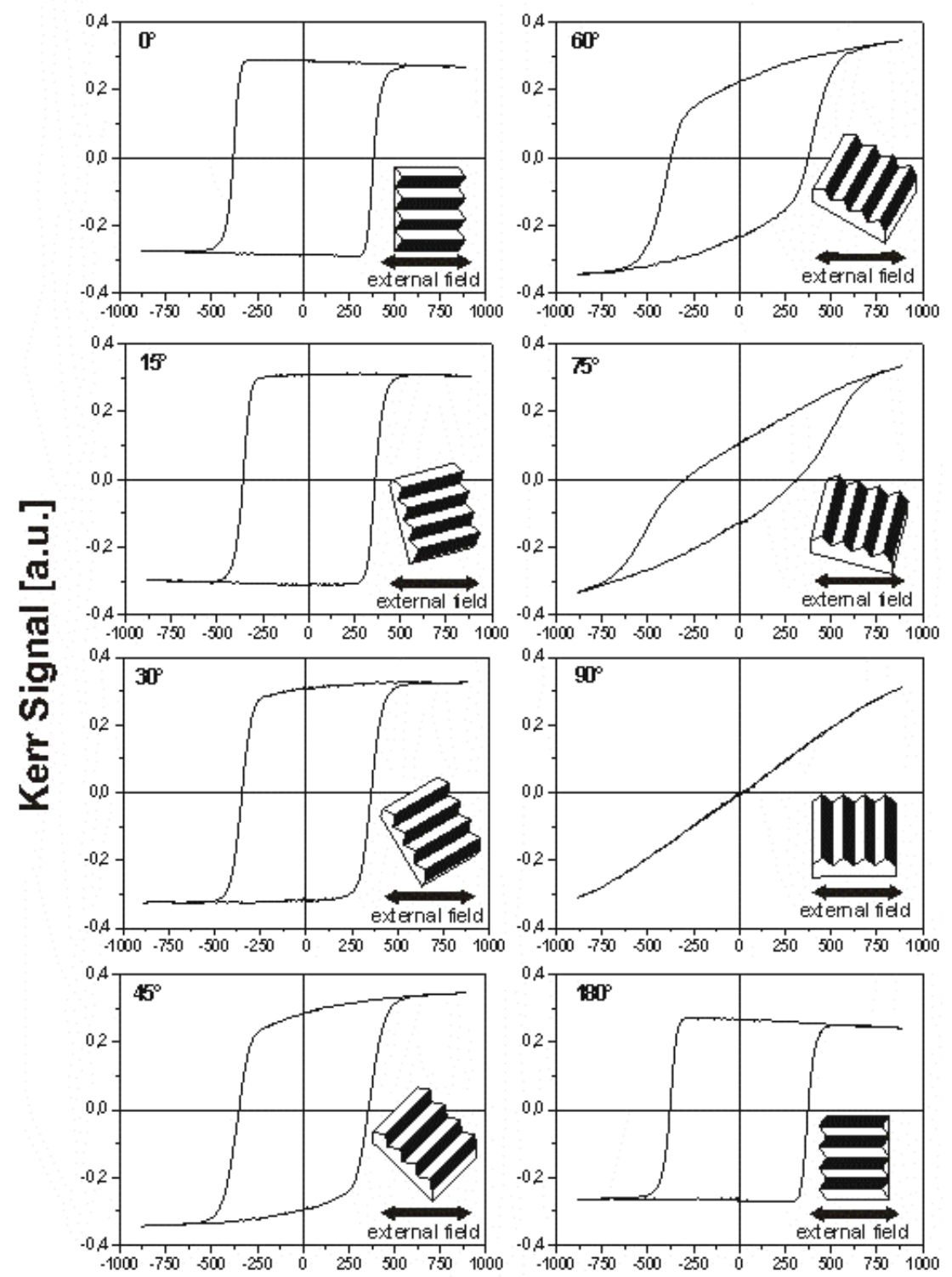

\section{$\mathrm{H}[\mathrm{Oe}]$}

Abbildung 40: Magnetisierungsverhalten (MOKE) eines Felds von EisenNanodrähten im wie hergestellten Zustand abhängig vom Winkel zwischen Facettierungsrichtung und äußerem Feld. 


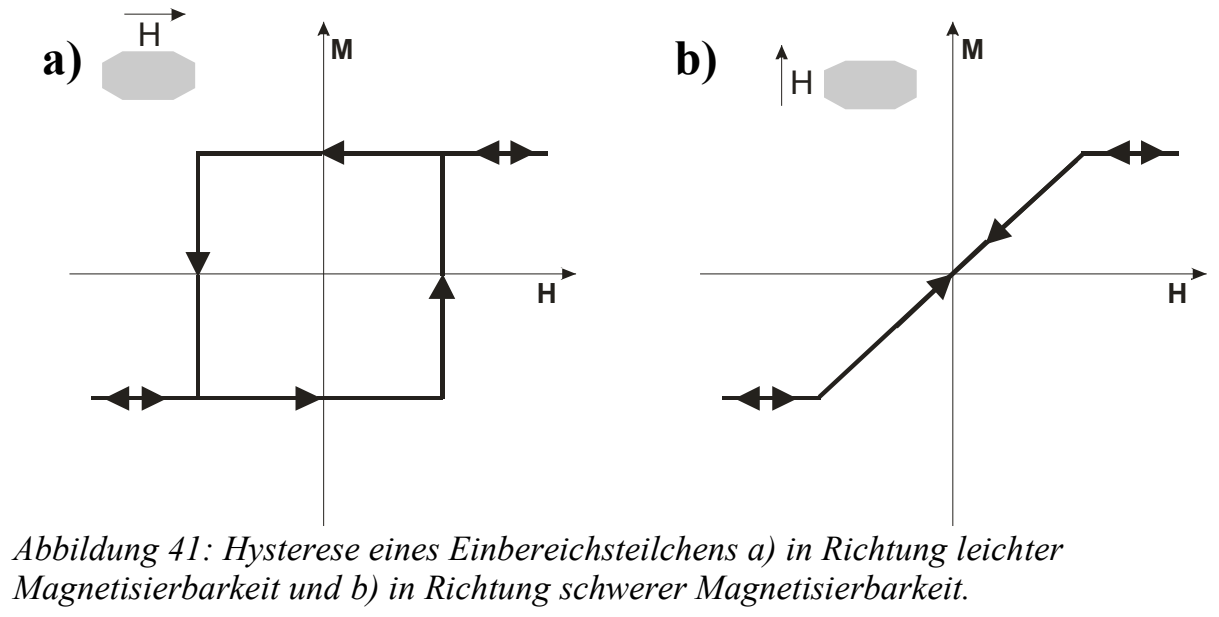

und Drahtrichtung wenig und noch bei $75^{\circ}$ wird ein Wert von $305 \pm 70$ e gemessen. Erst bei $90^{\circ}$ ist keine Koerzitivität aus der Magnetisierungskurve mehr messbar (Abbildung 40). Das große Entmagnetisierungsfeld verhindert eine Sättigung, so dass sich die Kurve auch noch im Bereich der höchsten Feldwerte stark der Symmetralen annähert.

Die magnetokristalline Anisotropie ist hier nur von untergeordneter Bedeutung. Durch Röntgendiffraktometrie konnte keine bevorzugte Orientierung, vor allem keine Epitaxie, festgestellt werden. Es ist bestenfalls von einer schwachen (110)-Drahttextur auf der Facettenfläche auszugehen.

Mit einer Schichtdicke von einigen Nanometern liegen die Drähte auch außerhalb des Spinreorientierungsübergang, bei dem die Magnetisierung mit Reduktion der Schichtdicke aus der Ebene dreht und schließlich parallel zur Substratnormalen ist. Dieser Größeneffekt wurde bei verschiedenen Materialien beobachtet, z.B. auch bei Eisen auf $\mathrm{Cu}(100)$ bei 3-4 Monolagen Bedeckung [Men 97], [Men 99], [Zil 94].

Ummagnetisierungsprozesse in ideal ferromagnetischen Teilchen, u.a. in unendlich langen Zylindern, wurden bereits 1957 von Frei et. al untersucht [Fre 57]. Wird ein äußeres Feld entlang der langen Achse eines Zylinders angelegt, so ist das Koerzitivfeld gleich dem Nukleationsfeld, wenn die magnetokristalline Anisototropie vernachlässigt werden kann. Das Koerzitivfeld nimmt dann mit zunehmendem Radius $R$ des Zylinders ab.

$$
H_{c}=6,78 \frac{A}{M_{s} \cdot R^{2}}
$$

Gleichung 6

Dabei ist $A$ die Austauschkonstante und $M_{s}$ das Sättigungsfeld.

Aharoni et al. zeigten, dass die Ummagnetisierung in einem unendlich ausgedehnten ferromagnetischen Zylinder in einem Sprung erfolgt, d.h. die Magnetisierungskurven eine rechteckige Form haben, und beschreiben den radiusabhängigen Übergang von sogenannten „,curling“ zum „,buckling“" [Aha 58].

Die im Rahmen dieser Arbeit präparierten Drähte haben nicht nur extreme Verhältnisse von Länge $(\sim 100 \mu \mathrm{m})$ zu Breite $(\sim 150 \mathrm{~nm})$, sondern auch von Breite zu Dicke $(<20 \mathrm{~nm})$, weshalb eine Betrachtung als Zylinder $\mathrm{zu}$ erheblichen Fehlern führen kann. In eindimensional lateral strukturierten Filmen, z.B. in Feldern von Nanodrähten, erwartet man Hysteresekurven mit ausgezeichneter leichter Richtung und mit großer Remanenz und Koerzitivität im Zusammenspiel mit einer starken in-plane Anisotropie [She 94], [Kum 96], [Sug 97]. Es ergeben sich aus diesen 
Aspektverhältnissen klare Verhältnisse für die Anisotropie. Durch die Formanisotropie wird, wie es von dünnen Schichten bekannt ist, die Magnetisierung in die Ebene gezwungen. Zusätzlich bewirkt die Formanisotropie durch das Breite-zu-Länge-Verhältnis der Drähte eine Ausrichtung in Facettierungsrichtung. Somit wird durch die Form der Nanodrähte eine uniaxiale Anisotropie induziert. Diese Interpretation entspricht der von Blundell et al, die im Fall einer Drahtstruktur eine uniaxiale Formanisotropie induziert sehen, deren leichte Richtung entlang des Drahtes und die schwere Richtung senkrecht dazu liegt. Wenn die Breite des Drahtes soweit reduziert wird, dass sie vergleichbar mit der Filmdicke wird, so sollte die Sättigungsmagnetisierung für die schwere Richtung in der Filmebene vergleichbar zum Sättigungfeld entlang der Filmnormalen werden [Blu 93]. Shearwood et al. untersuchten Anordnungen von lithographisch-ionenstrahlgeätzen Eisendrähten einer Breite von $0,5 \mu \mathrm{m}$, variierendem Drahtabstand von $0,5 \mu \mathrm{m}$ bis $16 \mu \mathrm{m}$ und einer Dicke von $25 \mathrm{~nm}$. Sie konnten zeigen, dass das Sättigungfeld in der Ebene senkrecht zu den Drähten im Bereich von $1 \mathrm{kG}$ liegt und damit höher als für einen durchgängigen Film $(0,3 \mathrm{kG})$, aber deutlich niedriger als das Sättigungfeld senkrecht zur Filmebene mit 22kG [She 94] ist.

Als relevanter Ummagnetisierungsprozess wird bei einer Orientierung des äußeren Feldes senkrecht zu den Drähten in der Ebene eine kohärente Rotation gefunden, unterstützt von den Beobachtungen von Oepen et al., die im Eisensystem eine Néel-Wandbreite von $210 \mathrm{~nm}$ angeben, was ca. der Hälfte der untersuchten Drahtbreite entspricht [Oep 89].

Im Gegensatz zu den vorgenannten Untersuchungen fanden Ressier et al. bei Eisen-Streifen, die durch epitaktische Abscheidung auf $\mathrm{MgO}(100)$ und anschließende plastische Deformation des gesamten Substrat-Schicht-Systems hergestellt wurden (,atomic saw method“), eine uniaxiale Anisotropie mit einer leichten Richtung senkrecht zu den Streifen [Res 97]. Die Streifenbreite variierte hierbei zwischen $50 \mathrm{~nm}$ und $2 \mu \mathrm{m}$, je nach lokaler Versetzungsdichte. Die Streifen verlaufen in Fe[110], die Eisenschichtdicke beträgt zwischen 2 und 5nm. Die Autoren nennen elastische Verspannungsfelder und deren Relaxation an den Gleitebenen als wahrscheinlichste Ursache für die ungewöhnliche Magnetisierungseinstellung. In den im Rahmen dieser Arbeit präparierten Nanodrähten liegt aber keine Grund für extreme Spannungszustände (durch epitaktische Verspannung, äußerer Krafteinwirkung, hochenergetische Beschichtungsverfahren, o.ä.) vor.

Die weite Öffnung der Hysterese zeigt das hartmagnetische Verhalten der Eisendrähte. Das spontane Schalten mit maximaler Sensitivität in Richtung der Facettierung erlaubt nur zwei Interpretationen. Entweder haben alle Drähte exakt dasselbe Koerzitivfeld oder aber die Drähte sind magnetostatisch gekoppelt, da sie wie ein zusammenhängender Film alle gleichzeitig schalten, [Sug 97].

Das Magnetisierungsverhalten der in dieser Arbeit untersuchten Felder von Eisennanodrähten ist von einer uniaxialen Formanisotropie in Facettierungsrichtung dominiert. Während bei einer parallelen Orientierung des magnetischen Feldvektors bezüglich der Längsachse der Drähte ein breite Hysterese mit einer starken Remanenz gemessen wird, ist bei einer senkrechten Einstellung das Magnetisierungsverhalten von reversibler Drehung geprägt. Wahrscheinlichliegt eine Kopplung der Drähte untereinander über eine statische Dipol-Kopplung $\left(\propto r^{-3} ; r\right.$ ist die Distanz parallel zur Drahtachse) vor, da der Abstand der Drähte deutlich kleiner ist als deren Länge. Das kollektive Verhalten des simultanen Ummagnetisierens der Drähte bei einheitlichem $\mathrm{H}_{\mathrm{c}}$ trotz der durch den Herstellungsprozess des Substrats bedingten Variation der Drahtbreite, legt das nahe. 


\subsubsection{Einfluss von Materialmenge und -verteilung am Beispiel Kobalt}

Die Menge des deponierten Materials hat bei der Deposition unter einem sehr flachen Winkel wesentlichen Einfluss auf die Gestalt der abgschiedenen Drähte. Insbesondere für die Umwandlung der Drähte in Reihen von separierten Partikeln ist das Materialvolumen der Drähte im Verhältnis zur Kontaktfläche zum Substrat von wesentlicher Bedeutung und entscheidet, ob eine einfache thermisch induzierte Gestaltumwandlung überhaupt möglich ist .

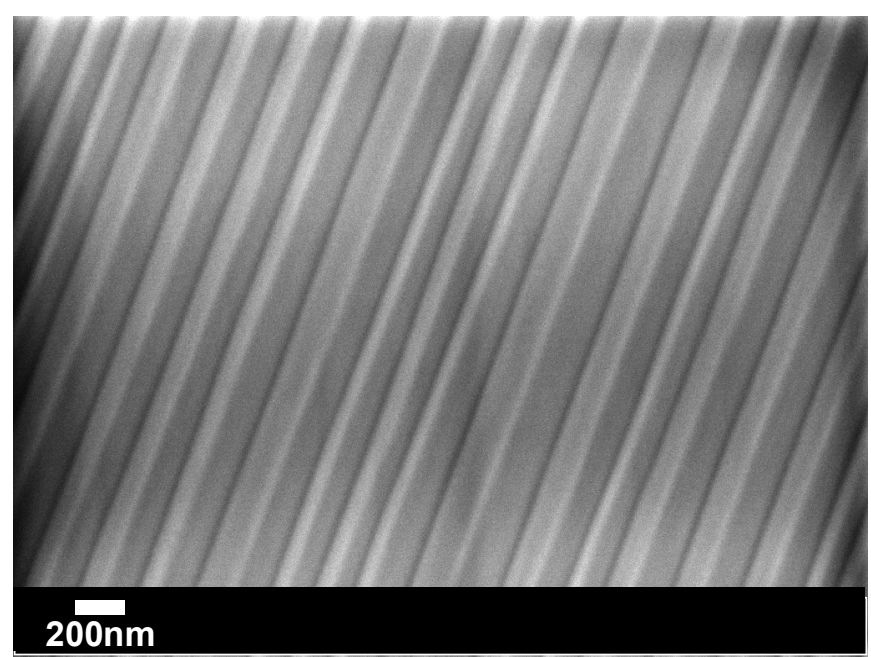

Abbildung 42: SEM-Aufsicht: $5 \mathrm{~nm}$ Kobalt Einzelschicht, senkrecht deponiert.
Untersuchungen zur Variation des Materialvolumens wurden am Beispiel des ferromagnetisch ordnenden Kobalts durchgeführt, da dieses aufgrund schwacher Diffusion auf der Oberfläche die geringste Neigung zeigt, die Facettenkämme zu überwachsen.

Kobalt wurde sowohl unter flachem Winkel (mittleren Dicken der Drähte von 5nm bzw. 20nm), wie auch senkrecht als Einzelschicht (ca. 5nm) auf das facettierte Substrat bei Raumtemperatur aufgebracht. Die unter senkrechter Geometrie aufgebrachte Schicht dient als Vergleich, um den Einfluss der Präformierung in Nanodrähte auf die thermisch induzierte Gestaltumwandlung bewerten zu können.

Abbildung 42 zeigt eine SEM-Abbildung einer ca. 5nm dicken Einzelschicht. Es sind keine Körner erkennbar, die Kontrastunterschiede zwischen den unterschiedlichen Facettenseiten sind gering. Die Leitfähigkeit der entstandenen Schicht ist vergleichsweise schlecht, was sich daran zeigt, dass die Beschleunigungsspannung bei der Abbildung im SEM auf $1 \mathrm{kV}$ reduziert werden musste, um Aufladeungseffekte in einem vertretbaren Rahmen $\mathrm{zu}$ halten. Insgesamt kann von einer vergleichsweise homogenen, sehr dünnen Bedeckung ausgegangen werden.

Die Untersuchung einer entsprechenden Probe mit dem MFM bestätigt die Bedeckung mit einer dünnen ferromagnetischen Schicht. Abbildung 43 zeigt Höhen- (a) und Phaseninformation (b). Zur topologischen Abbildung (a) ist zusätzlich ein Schnitt entlang der weißen Linie gegeben. Ein Vergleich mit der rasterelektronenmikroskopischen Aufnahme (Abbildung 42) zeigt, dass das Material nicht vollständig homogen auf den Facetten wächst. Die Schnittdarstellung verdeutlicht, dass die Deposition zu einer Verbreiterung der Facettenspitze führt und somit eine stärkere Materialanlagerung in diesem Bereich eine Abrundung der Facettenkämme zur Folge hat, während die Täler unveränderte Facettierungswinkel zeigen. In der Phasendarstellung (b) dominiert ebenfalls die Topologie der facettierten Oberfläche, jedoch ist die Wechselwirkung der ferromagnetischen Spitze mit dem Streufeld der Probe erkennbar. Die magnetischen Domänengrenzen der Schicht sind als „wolkige“ Struktur der Facettierung überlagert. Es ist kein deutlicher Zusammenhang zwischen dem Verlauf der Domänengrenzen und der Facettierung erkennbar. 

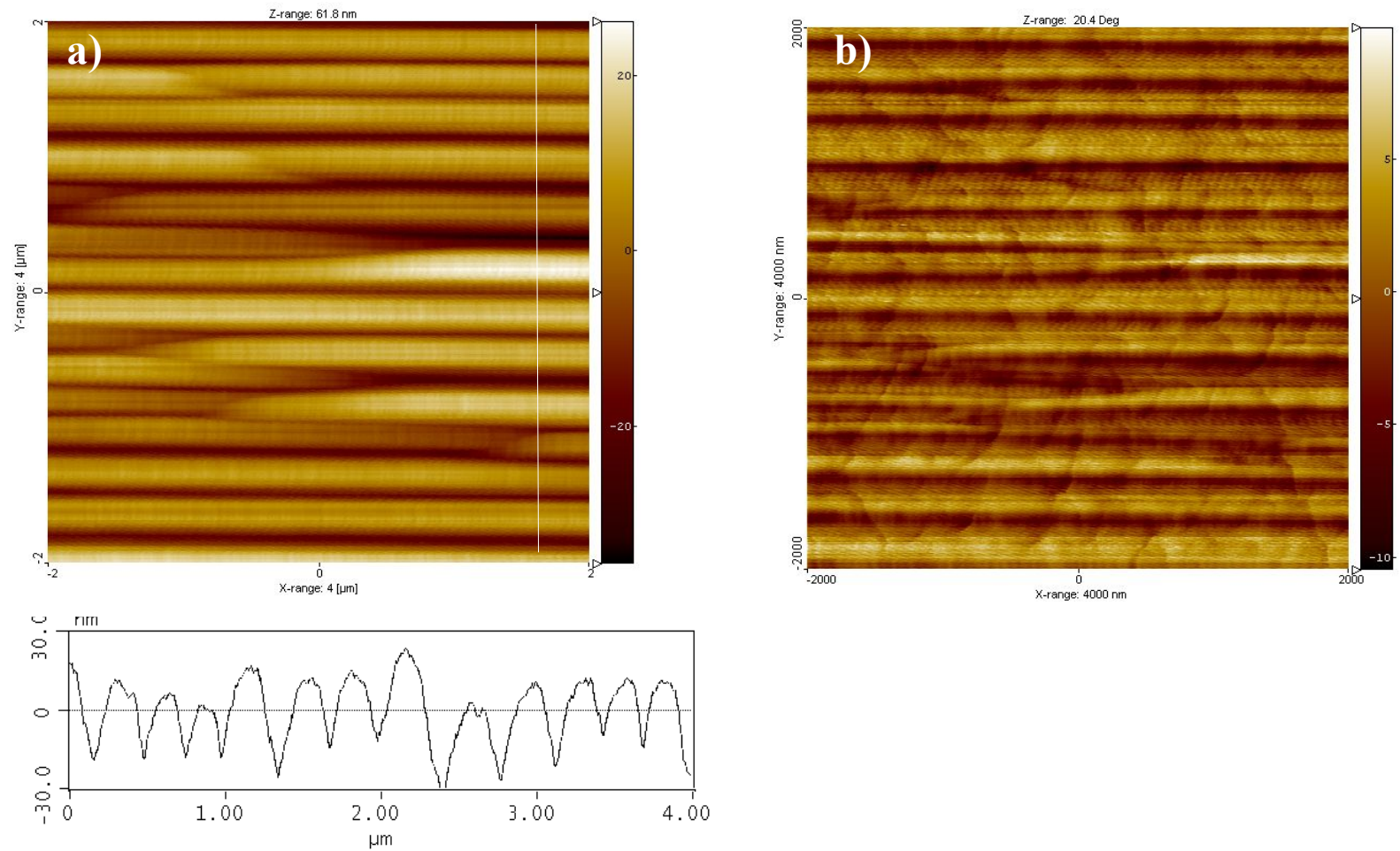

Abbildung 43: a) MFM-Höhendarstellung mit Schnitt entlang der weißen Linie und b) Phasensignal einer ca. $5 \mathrm{~nm}$ Einzelschicht Co auf facettiertem $\mathrm{Al}_{2} \mathrm{O}_{3}$, Probe nicht magnetisiert.

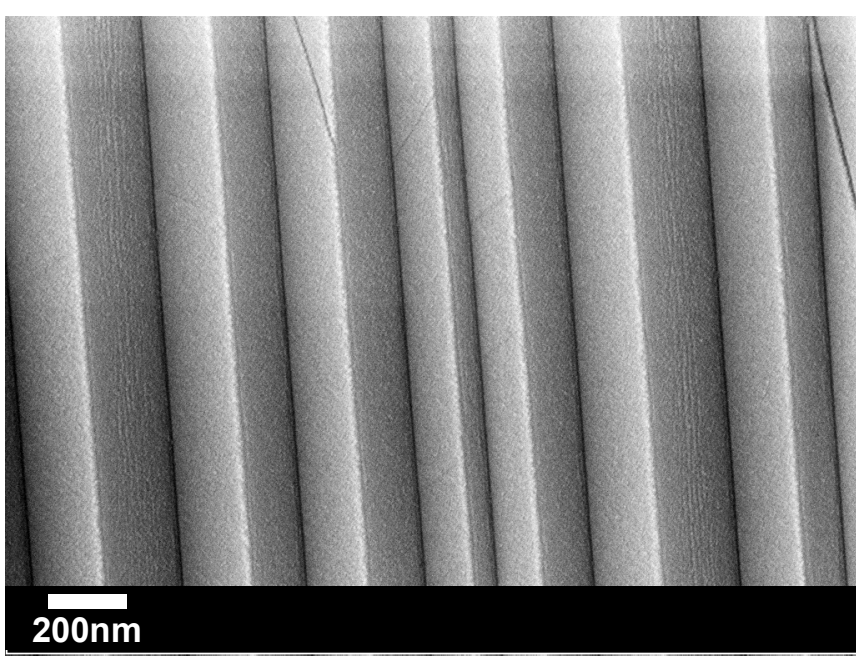

Abbildung 44: SEM-Aufsicht: ca. 5nm Kobalt, flache Depositon, Depositionssrichtung von links nach rechts.

Eine vergleichbare Schichtdicke Kobalt wurde durch Deposition auf eine facettierte Saphiroberfläche unter flachem Winkel aufgesputtert (siehe Abbildung 44). Die Deposition erfolgte von der linken Seite aus. Die dem Materialstrom zugewandten Facettenseiten zeigen einen variierenden Kontrast von dunkel im Tal nach hell am Facettenkamm. Der ausgeprägt helle Kontrast am Facettenkamm ist typisch für Kanten Sekundärelektronenabbildungen. Zusätzlich erkennt man auf den Schattenseiten mehrere dünne, sehr helle Linien. Hierbei handelt es sich um die Nanofacetten. Wie schon in Kapitel 3.2 erwähnt, sind die beiden Facettenseiten nicht äquivalent, d.h. während es sich bei der einen Seite um eine (atomar) glatte $(10 \overline{1} 1)$-Fläche handelt, ist die andere zusammengesetzt aus vielen kleinen Stufen. Diese werden durch das unter flachem Winkel geputterte Kobalt besonders gut sichtbar.

Obwohl weder mittels SEM noch mittels MFM eine deutliche präferentielle Bedeckung einer Facettenseite $\mathrm{zu}$ beobachteten ist, zeigt sich ein vergleichbares, ferromagnetisches Verhalten, wie bei den Eisendrähte. Aus der Aufwachsrate kann die Obergrenze der Bedeckung mit 5nm Kobalt angegeben werden. 
a)

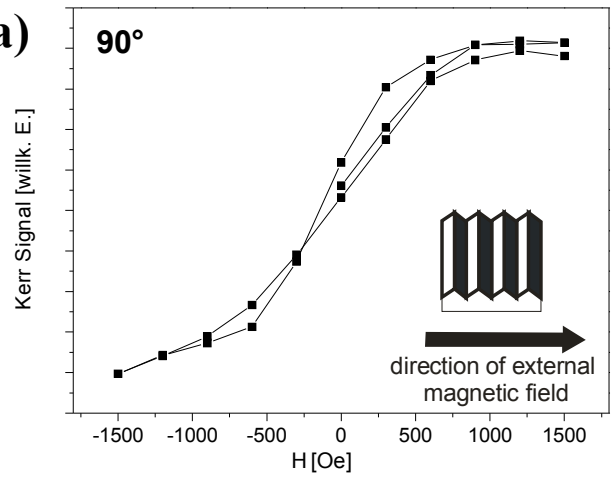

b)

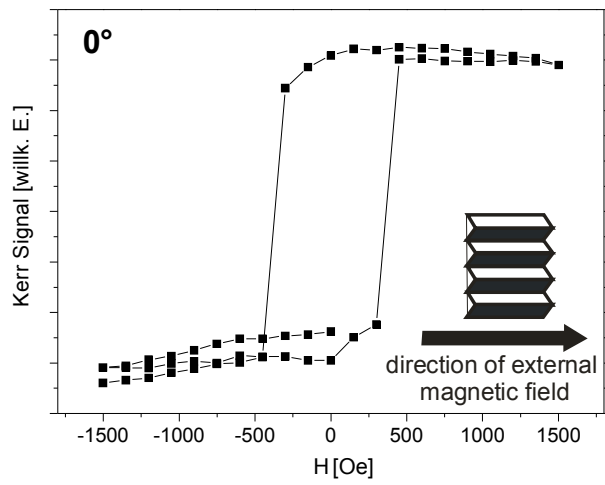

Abbildung 45: MOKE: Magnetisierungverhalten von sehr dünnen Co-Drähten (ca. 5nm) auf facettiertem $\mathrm{Al}_{2} \mathrm{O}_{3}$, wie hergestellt; a) senkrecht und b) parallel zur Facettierungsrichtung.

Abbildung 45 zeigt die Daten von MOKE-Messungen an diesen dünnen Co-Drähten im Zustand direkt nach der Präparation.Die bereits für die Eisendrähte diskutierte Anisotropie zwischen dem Magnetisierungsverhalten senkrecht (a) und parallel (b) zu den Drähten ist deutlich erkennbar.

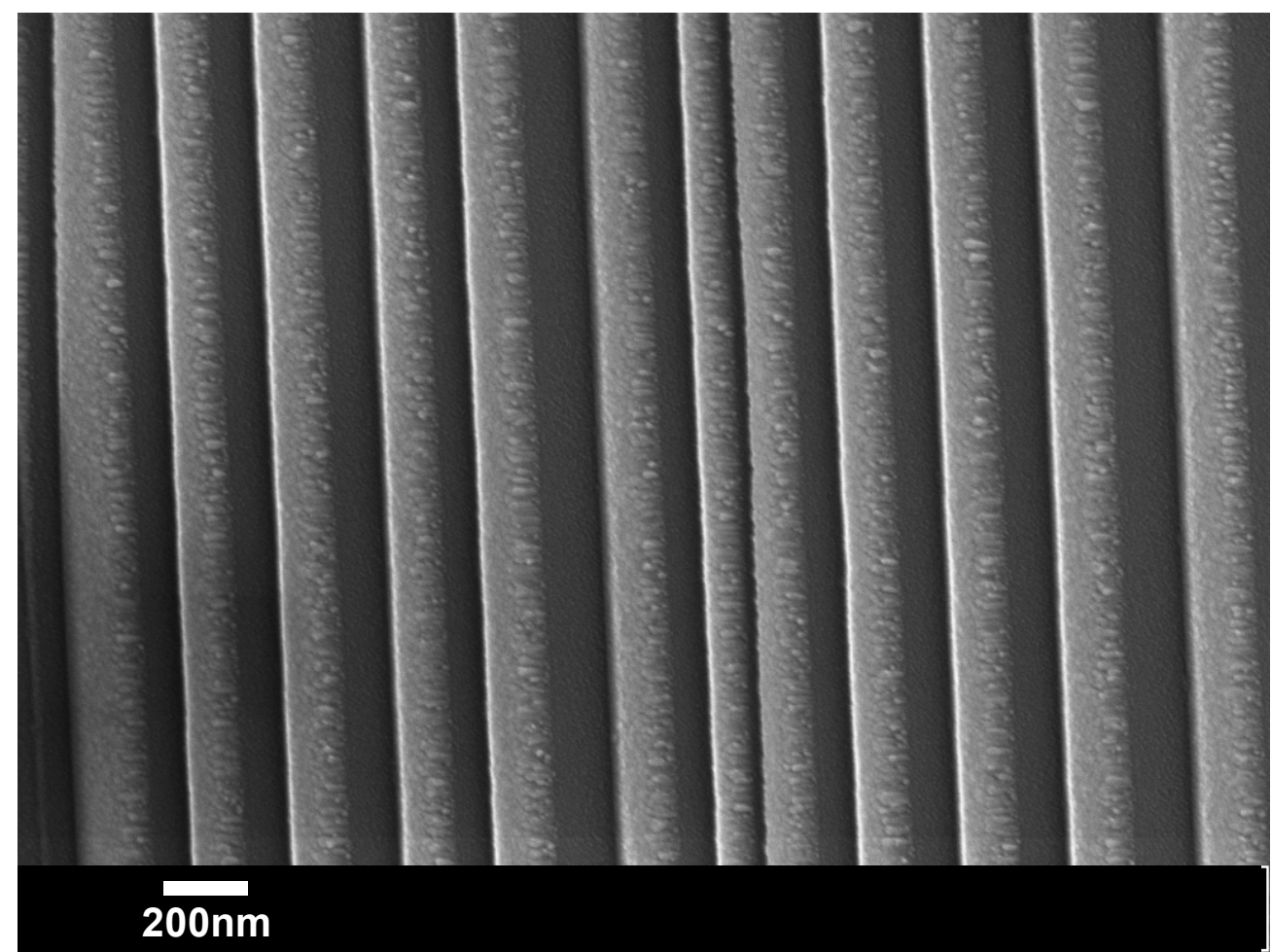

Abbildung 46:SEM-Aufsicht von ca. 20nm Co-Drähten, wie hergestellt, Depositionsrichtung von links nach rechts.

Vergrößert man die nominell deponierte Schichtdicke auf 20nm, so ändert sich die beobachtete Morphologie (Abbildung 46). Es sind Körner sichtbar, deren Position mit den Facettenkämmen identifiziert werden kann. Die dem Materialstrom zugewandte Seite zeigt einen hellen, die abgeschattete einen dunklen Kontrast. Die Körner auf den Facettenkämmen sind, wie im Fall des Eisens, in Depositionsrichtung gestreckt mit vergleichbaren Aspektverhältnissen. 
Durch die Steigerung der Schichtdicke lässt sich die bevorzugte Bedeckung einer Facettenseite nun auch mittels AFM nachweisen (Abbildung 47). Abbildung 47b zeigt einen Schnitt, der in der Mitte von Bild Abbildung 47a erstellt wurde. Am Fuß einiger Facetten ist in Bereichen das nahezu unbedeckte Substrat erkennbar. Dies wird durch einen einige Nanometer großen Bereich konstanter

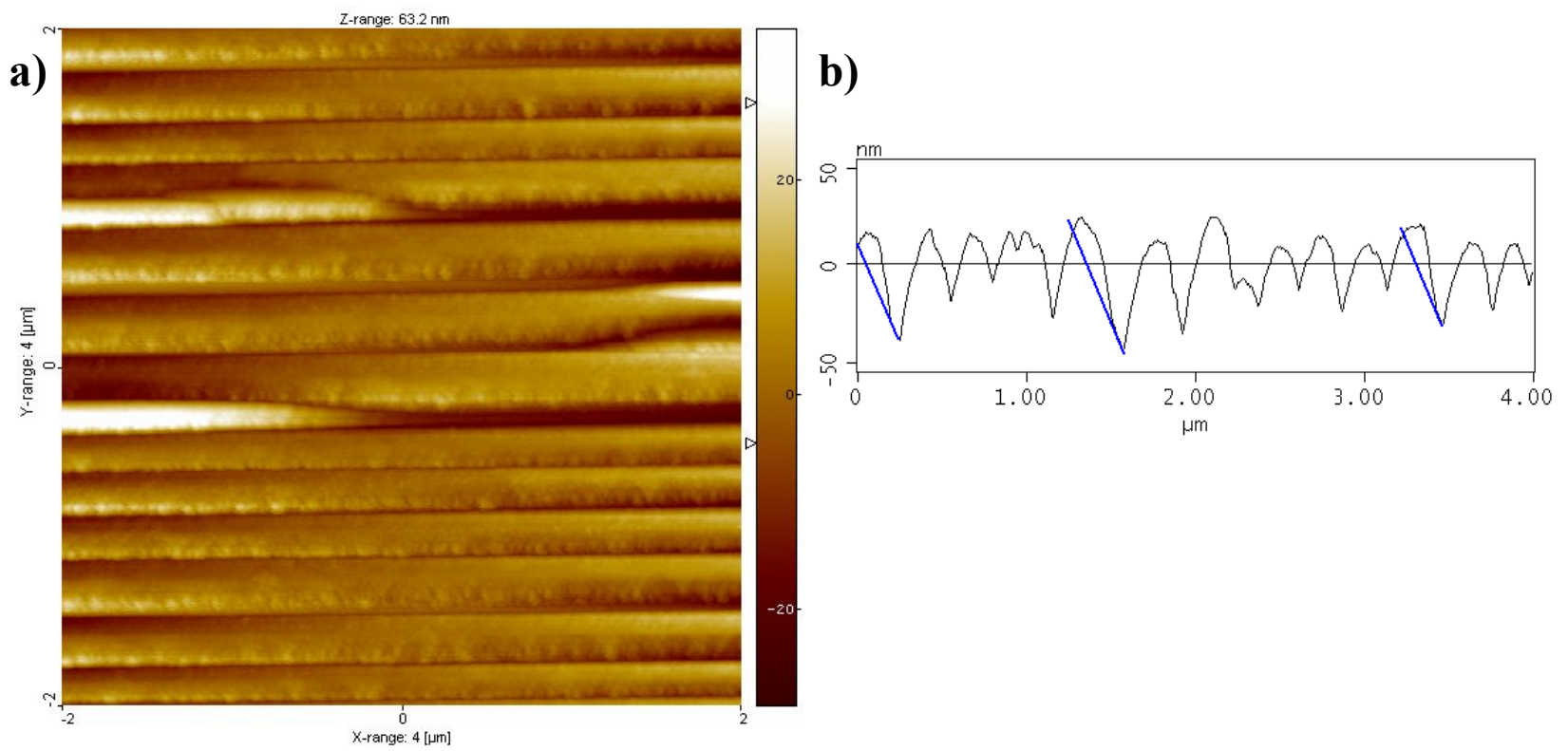

Abbildung 47: a) AFM-Höheninformation von ca. 20nm Co-Drähten wie hergestellt und b) zugehörige Schnitt darstellung. Die blaue Linien markieren den Facettenseitenverlauf.

Steigung deutlich. Eine Extrapolation dieser Steigung in Richtung der Facettenspitze (blaue Linien) gibt einen sinnvollen Anhaltspunkt für den Verlauf der Grenzfläche zwischen Substrat und Kobaltschicht. Somit wird auch die Verteilung des Kobalts nach der Depositon auf der Facettenseite deutlich. Durch die Abschattung wird der größte Teil des Materials im Bereich der Spitzen deponiert, die Schichtdicke reduziert sich in Richtung der Täler bis hin zu den vereinzelt auftretenden Extremfällen, in denen am Fuß der Facette nahezu keine Bedeckung vorhanden ist. Die vorhandene Abrundung der anderen Facettenseite im Bereich der Spitze deutet auf eine geringes Überwachsen der Spitzen bzw. eine leichte Bedeckung der abgeschatteten Facettenseiten im Bereich der Spitzen hin.

a)

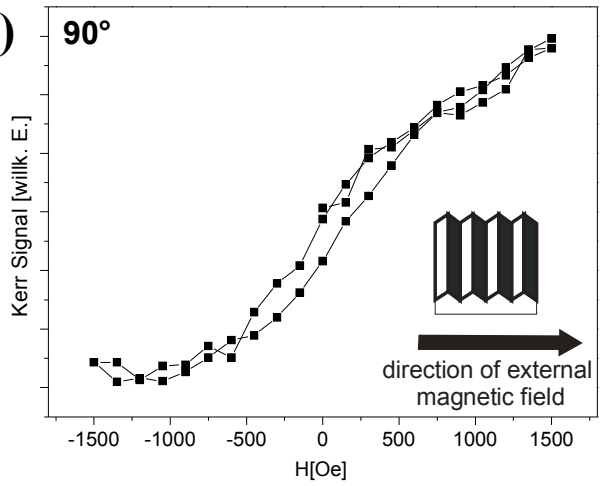

b)

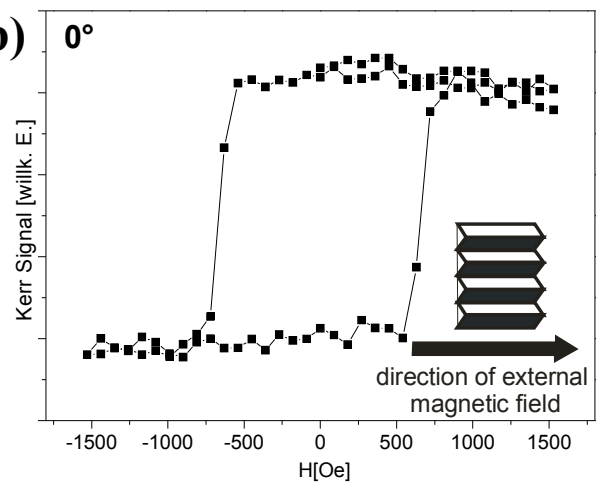

Abbildung 48: MOKE: Magnetisierungverhalten von ca. 20nm dicken Co-Drähten, wie hergestellt. a) senkrecht und b) parallel zu Facettierungsrichtung. 
Durch die größere Schichtdicke vergrößert sich der Querschnitt der Drähte und ein Auswirkung auf die ferromagnetischen Eigenschaften sind denkbar. Es wird ein Verbreiterung der Hysterese in Feldrichtung parallel zu den Drähten beobachtet (vergleiche Abbildung 45b und Abbildung 48b). Das Koerzitivfeld verdoppelt sich annähernd. Das magnetisch anisotrope Verhalten, bedingt durch die eindimensionale Form, ist auch bei den dickeren Drähten offensichtlich. Dabei ist zu berücksichtigen, dass die Drahtdicke im Vergleich zur Breite sehr gering ist und daher auch nicht durch eine zylindrische Form im Sinne von Gleichung 6 (siehe Seite 44) beschrieben werden kann. Ein magnetisch gekoppeltes Verhalten ist auch bei den Kobaltdrahtfeldern durch sehr scharfe Übergänge bei $\mathrm{H}_{\mathrm{c}}$ direkt zur Sättigungsmagnetisierung, sowie $\mathrm{M}_{\mathrm{r}} / \mathrm{M}_{\mathrm{s}}=1$ nahegelegt.

Zusammenfassend kann gesagt werden, dass es gelungen ist die periodisch geordneten Facetten des Substrats zu nutzen, um mittels flacher Deposition die Periodizität in Felder von Nanodrähten aus Eisen und Kobalt mit Drahtbreiten im Bereich der Facettenseitenbreite $(\sim 160 \mathrm{~nm})$ zu übertragen. In-situ Leitwertmessungen während der Eisendeposition legen eine geringe Bedeckung der Schattenseite der Facetten nahe und zeigen Perkolation für die Drahtseite bei einer nominellen Schichtdicke von $\mathrm{d}>4,5 \pm 1,5 \mathrm{~nm}$. Das Magnetisierungsverhalten der Felder ist geprägt von uniaxialer Formanisotropie und lässt ein magnetostatische Kopplung der Drähte vermuten. 


\subsection{Gestaltumwandlung durch Temperaturbehandlung}

Im Folgenden werden die Untersuchungen zur Gestaltumwandlung der metallischen Drähte beschrieben, durch die die Nanodrähte schließlich in Reihen von separierten, magnetischen Partikeln überführt werden können. Während der Temperaturbereich der Umwandlung mit in-situ Widerstandsmessungen sichtbar gemacht werden konnte, eignet sich insbesondere die Rasterelektronenmikroskopie und die Rasterkraftmikroskopie zur Charakterisierung der Veränderung der Oberflächenmorphologie sowie der Clustererzeugung aus den zusammenhängenden Drähten. Zudem sollte die in den Magnetisierungsmessungen auftretende starke Anisotropie bei einer Bildung separierter runder Körner verschwinden.

\subsubsection{Stadien der Gestaltumwandlung am Beispiel der Eisennanodrähte}

Nach der Deposition erfolgte in-situ, d.h. ohne Brechen des Vakuums (Restgasdruck: $3 \cdot 10^{-7}-5 \cdot 10^{-9} \mathrm{mbar}$ ) und Veränderungen an der Kontaktierung, eine Temperbehandlung der Proben (mit einer konstanten Heizrate von $3 \mathrm{~K} / \mathrm{min}$ ).

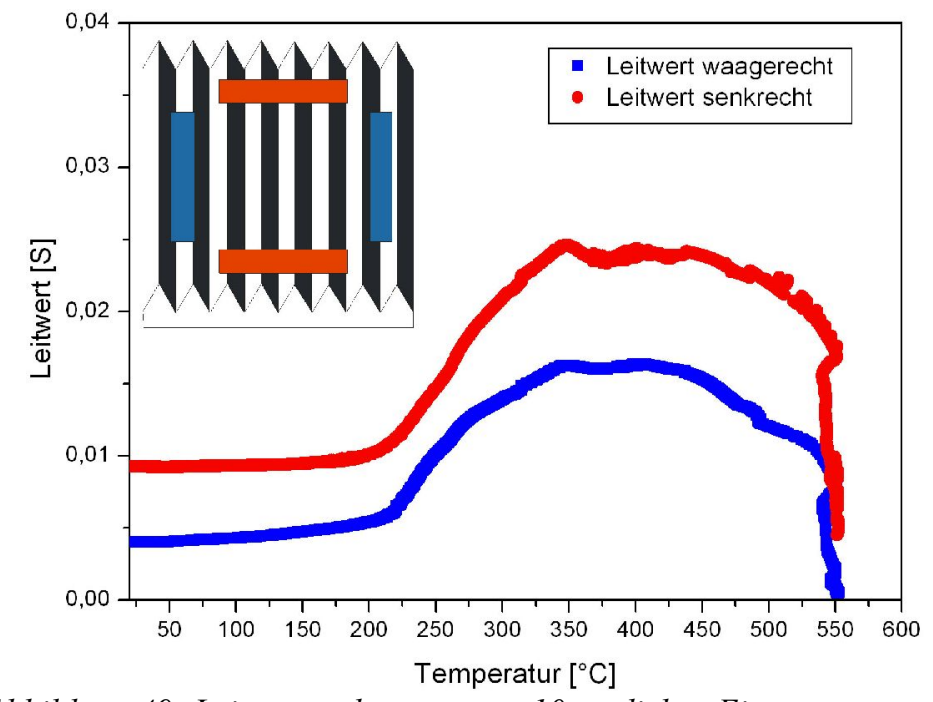

Abbildung 49: Leitwertänderung von 10 nm dicken EisenNanodrähten während der Temperung bis $550^{\circ} \mathrm{C}$ auf facettiertem $\mathrm{Al}_{2} \mathrm{O}_{3}$.

Beide Messrichtungen zeigen eine vergleichbare Entwicklung im Leitwert mit steigender Temperatur (Abbildung 49). Im niedrigen Temperaturbereich unterhalb von ca. $205^{\circ} \mathrm{C}$ bleibt der Leitwert weitgehend unverändert. Es schließt sich ein Bereich zwischen $205^{\circ} \mathrm{C}$ und $348^{\circ} \mathrm{C}$ an, in dem der Leitwert um einen Faktor 2,5-3,5 steigt. In diesem Bereich reduziert das Ausheilen von Defekten die Streuung der Leitungselektronen. Bis $450^{\circ} \mathrm{C}$ bleibt der Leitwert konstant durch die gegensätzlichen Einflüsse der beiden Prozesse der Verbesserung der Leitung durch Defektreduktion und der erhöhten Streuung an thermischen Phononen. Erst bei $540^{\circ} \mathrm{C}$ bricht der Leitwert in beiden Messrichtungen ein.

Während senkrecht zur Facettierungsrichtung bei $550^{\circ} \mathrm{C}$ sehr schnell keine Leitfähigkeit mehr messbar ist, verbleibt bei der Messrichtung entlang der Drähte in Facettierungsrichtung (rote Punkte Abbildung 49) eine Leitfähigkeit und eine weitere, geringe Temperatursteigerung auf $560^{\circ} \mathrm{C}$ ist zur vollständigen Unterbrechung des Transports notwendig.

Durch die temperaturabhängige Leitwertmessung lässt sich der für die Gestaltumwandlung relevante Temperaturbereich also auf einen Bereich von $450^{\circ} \mathrm{C}$ bis über $560^{\circ} \mathrm{C}$ festlegen. Dabei ist zu erwarten, dass im Bereich niedriger Temperaturen erste Veränderungen in der Mikrostruktur der Drähte sichtbar werden, die möglicherweise Rückschlüsse auf den Zerfallsmechanismus zulassen, bevor schließlich ab $550^{\circ}-560^{\circ} \mathrm{C}$ ein Zerfall der Drähte stattfindet. Die Temperaturbehandlung von 
Eisen-Nanodrähten nur wenig über der Temperatur, für die die Unterbrechung des Stromtransportes festgestellt wurde, sollte zu einer schnellen Umformung führen, da sich Temperatursteigerungen über das Arrheniusgesetz exponentiell auf die Diffusion auswirken.
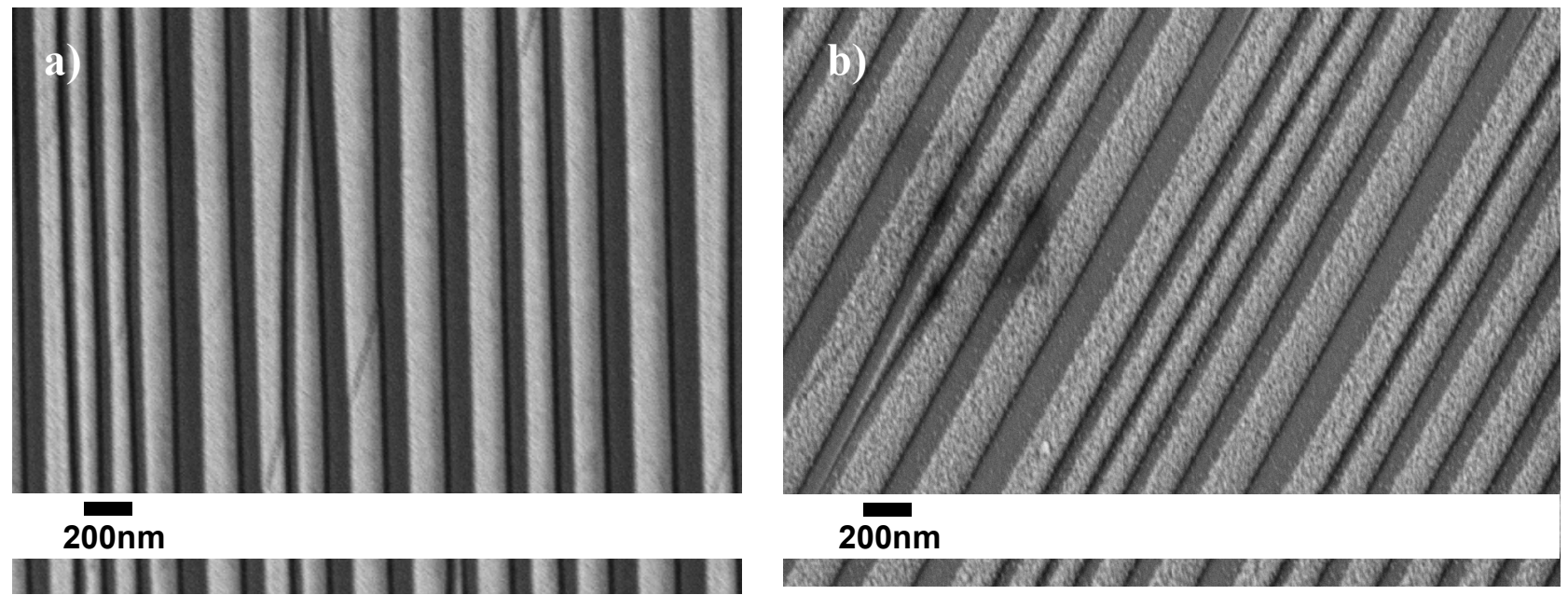

Abbildung 50: SEM-Analysen: Vergleich von 10 nm dicken Fe-Drähten a) nicht getempert und b) von noch zusammenhängenden Fe-Drähten nach Temperung für $1 \mathrm{~h}$ bei $400^{\circ} \mathrm{C}$. Die hellere, rechte Seite ist mit Eisen belegt.

Um die Frühstadien des Zerfalls der Drähte beobachten zu können, wurden oberflächensensitive Untersuchungen zunächst an Proben durchgeführt, die deutlich unter der in den Leitwertmessungen ermittelten Zerfallstemperatur getempert wurden.

Eine Temperung für $1 \mathrm{~h}$ bei $400^{\circ} \mathrm{C}$ bei einem Restgasdruck von $1,9 \cdot 10^{-7} \mathrm{mbar}$ führt zu einer deutlichen Aufrauung der Eisenoberfläche. Die SEM Untersuchungen zeigen aber keine Unterbrechungen der Drähte. Der Vergleich der Darstellungen in Abbildung 50 zeigt, dass es durch die Temperung zu einer deutlichen Aufrauung der Eisenoberfläche gegenüber dem Zustand nach der Herstellung gekommen ist. Daneben finden sich aber keine Anzeichen für eine Veränderung der lateralen Form der Drähte, z.B. im Sinne von regelmäßigen oder auch unregelmäßigen Modulationen der Drahtbreite oder Einschnürungen im Bereich von Fehlern der Oberflächenfacettierung des Substrats.
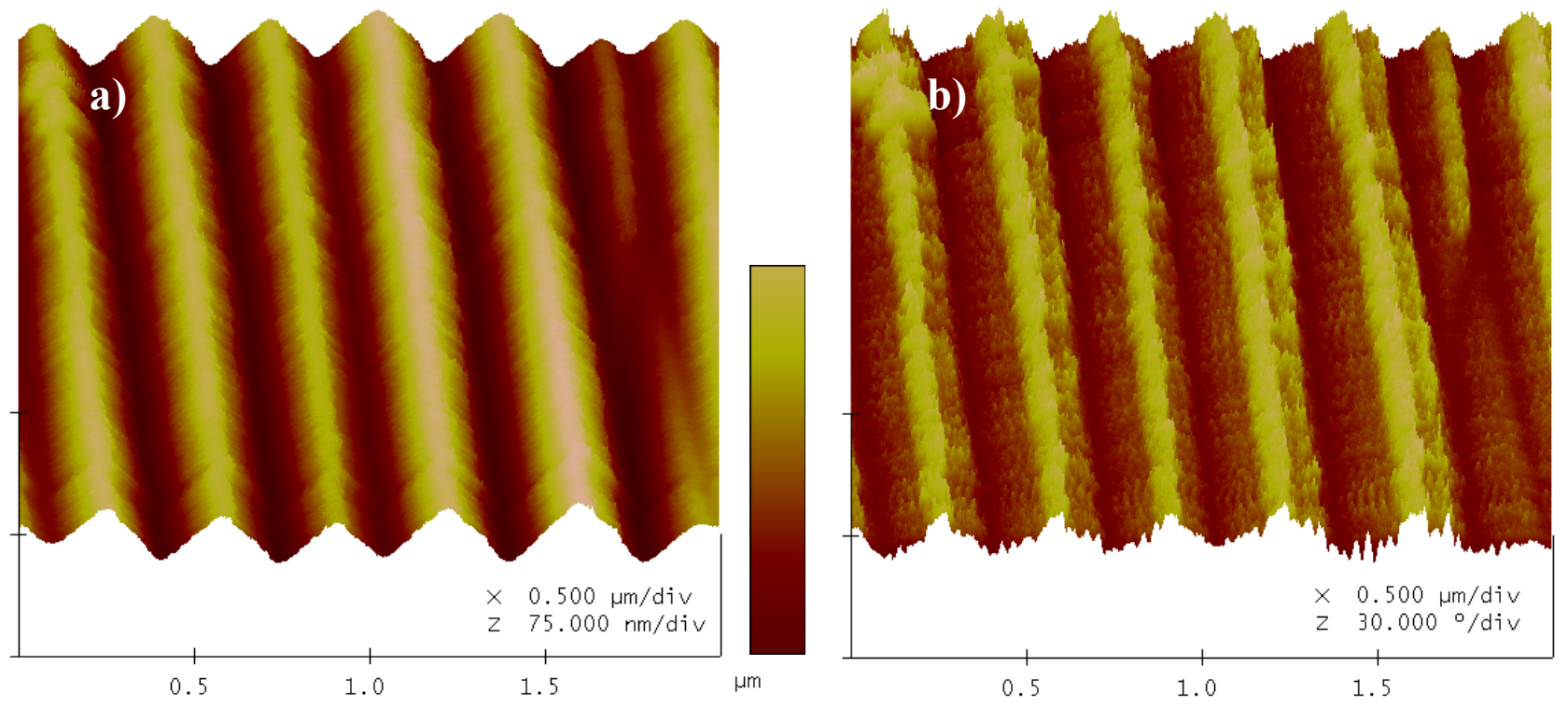

Abbildung 51: a) MFM-Höhen- und b) Phasensignal von $\sim 10 \mathrm{~nm}$ dicken Eisendrähten nach $1 \mathrm{~h}$ bei $400^{\circ} \mathrm{C}$. Der Vergleich zeigt den Phasenkontrast nur auf der rechten Facettenseite, z-Skala: $75 \mathrm{~nm}$ bzw. $30^{\circ}$. 
MFM-Untersuchungen an diesen bei $400^{\circ} \mathrm{C}$ getemperten Drähten zeigen ebenso wie nicht getemperte Drähte, dass nur von einer der Facettenseiten ein magnetisches Signal beobachtet wird. Die Drähte wurden unmittelbar vor der Untersuchung mit dem MFM in einem Magnetfeld in der Filmebene aufmagnetisiert, dabei zeigen Versuche mit Magnetfeldrichtungen senkrecht und parallel zur Facettierungsrichtung keine wesentlichen Unterschiede im magnetischen Phasenkontrast. Abbildung 51 zeigt die simultan erzeugten Höhen- und Phasenkontrastbilder. Im Höhenkontrast (Abbildung 51a) ist die regelmäßig, gleichseitige Facettierung gut nachvollziehbar. Es fällt auf, dass sich im Phasenkontrastbild (Abbildung 51b) breite Streifen niedriger Intensität mit Doppelstreifen aus hoher und niedriger Intensität abwechseln. Der Kontrastwechsel der Doppelstreifen kommt durch die

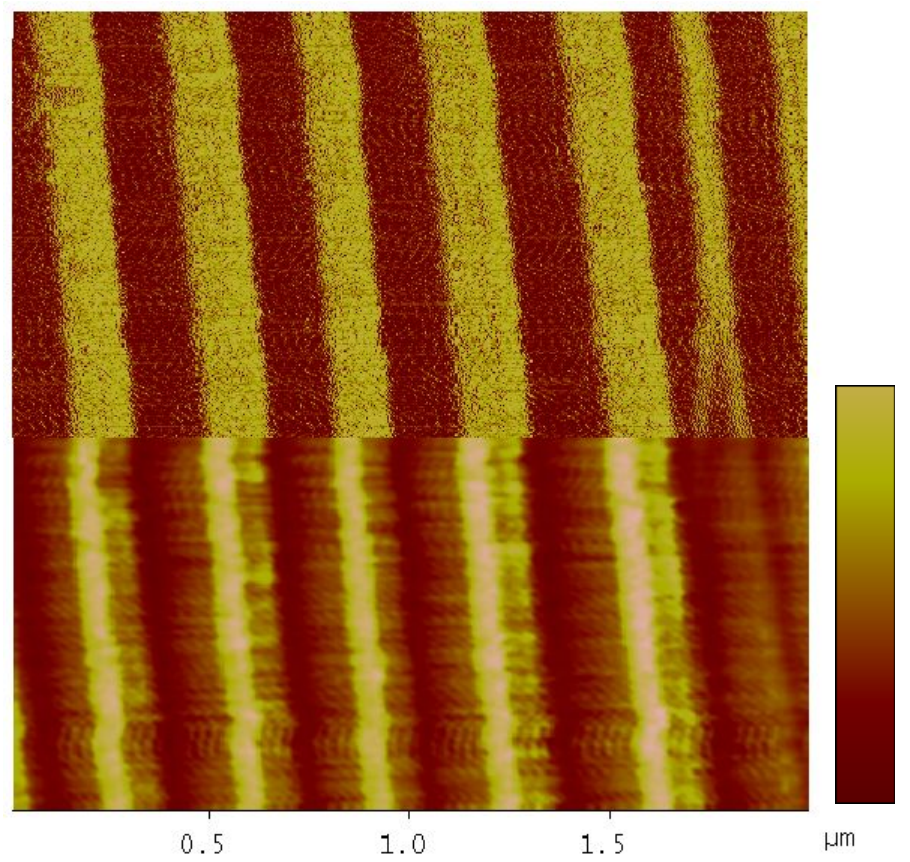

Abbildung 52: MFM-Höhensignal (Steigungskontrast)(oben) und Phasensignal (unten) von $\sim 10 \mathrm{~nm}$ dicken Eisendrähten nach $1 \mathrm{~h}$ bei $400^{\circ} \mathrm{C}$, ortsrichtiger Zusammenschnitt, z-Skala: $75 \mathrm{~nm}$ bzw. $15^{\circ}$. komplexe Wechselwirkung der magnetischen Spitze mit dem Streufeld der ferromagnetischen Streifen zustande. Die Interpretation der Kontrastwechsel ist nicht trivial, da sowohl der Gradient $\nabla H$ als auch der quadratische Gradient $\nabla^{2} H$ und das Streufeld $H$ selbst in die Phasenverschiebung eingehen (vgl. Kapitel 2.4 Rasterkraftmikroskopie).

Um die Lage der Doppelstreifen bezüglich der Facetten besser einordnen zu können, ist in Abbildung 52 ein ortsrichtiger Zusammenschnitt von Phasenbild und einer Höhendarstellung zu sehen. Dabei wurde, um einen großen Kontrast zwischen den unterschiedlichen Facettenseiten der Höhendarstellung zu erreichen, eine Darstellung mit einer imaginären Lichtquelle von rechts gewählt. Folglich sind die der Quelle zugewandten, linken Seiten hell, die abgewandten, rechten dunkel, es entsteht also ein Steigungskontrast. Der Zusammenschnitt zeigt, dass die Doppelstreifen im Phasenbild auf der linken (hellen) Facettenseite lokalisiert sind.

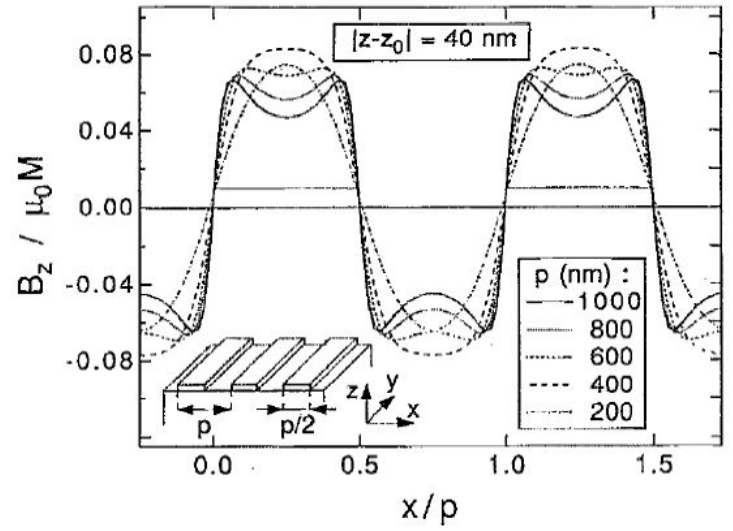

Abbildung 53: z-Komponente des magnetischen Feldes einer periodischen Drahtanordnung mit der Periode $p$ und der Drahtbreite $\mathrm{p} / 2$ im Abstand von 40nm. Drahtdicke: 50nm (aus [Roy 92]).
Für die Untersuchungen mit einer magnetischen Spitze ist die Wechselwirkung des Streufeldes der Proben mit dem magnetischen Moment der Spitze entscheidend. Das Problem eines äquidistanten Drahtfeldes mit konstanter Periode, das dem Problem der Drähte auf Facetten sehr nahe kommt, wurde von van Roy et al. untersucht [Roy 93]. Die Stärke des Streufeldes eines Drahts ist vom Verhältnis von lateraler Ausdehnung und Drahtdicke bestimmt. Daher benötigt man bei typischen Schichtdicken der Dünnschichttechnologie von 10-100nm auch Strukturbreiten im Bereich $>100 \mathrm{~nm}$, um ein hinreichendes Entmagnetisierungsfeld zu erreichen.

Bei einer Drahtdicke von 50nm konnten van Roy 
et al. ein Maximum im Entmagnetisierungsfeld von (nur) 8\% der Magnetisierung des Drahtes im Abstand von 40nm bei einer optimalen Periode der Drähte von 400nm erreichen. Die optimale Periode skaliert mit der Drahtdicke (Abbildung 53). Diese Untersuchungen machen einerseits deutlich, dass ein geringer Abstand zur Probe wichtig ist, um eine hohe Signalstärke zu erhalten, andererseits durch Wahl der optimalen Periodizität eine Maximierung des Streufeldes in einer festen Höhe über der Probe zu erreichen ist [Roy 92].

Insgesamt ist festzuhalten, dass der magnetische Phasenkontrast der MFM-Analysen nach einer Auslagerung bei $400^{\circ} \mathrm{C}$ die Periodizität der Drahtanordnung nach der Herstellung zeigt. Es gibt weder topologisch noch magnetisch Anzeichen für einen Zerfall der Drähte in Partikel.

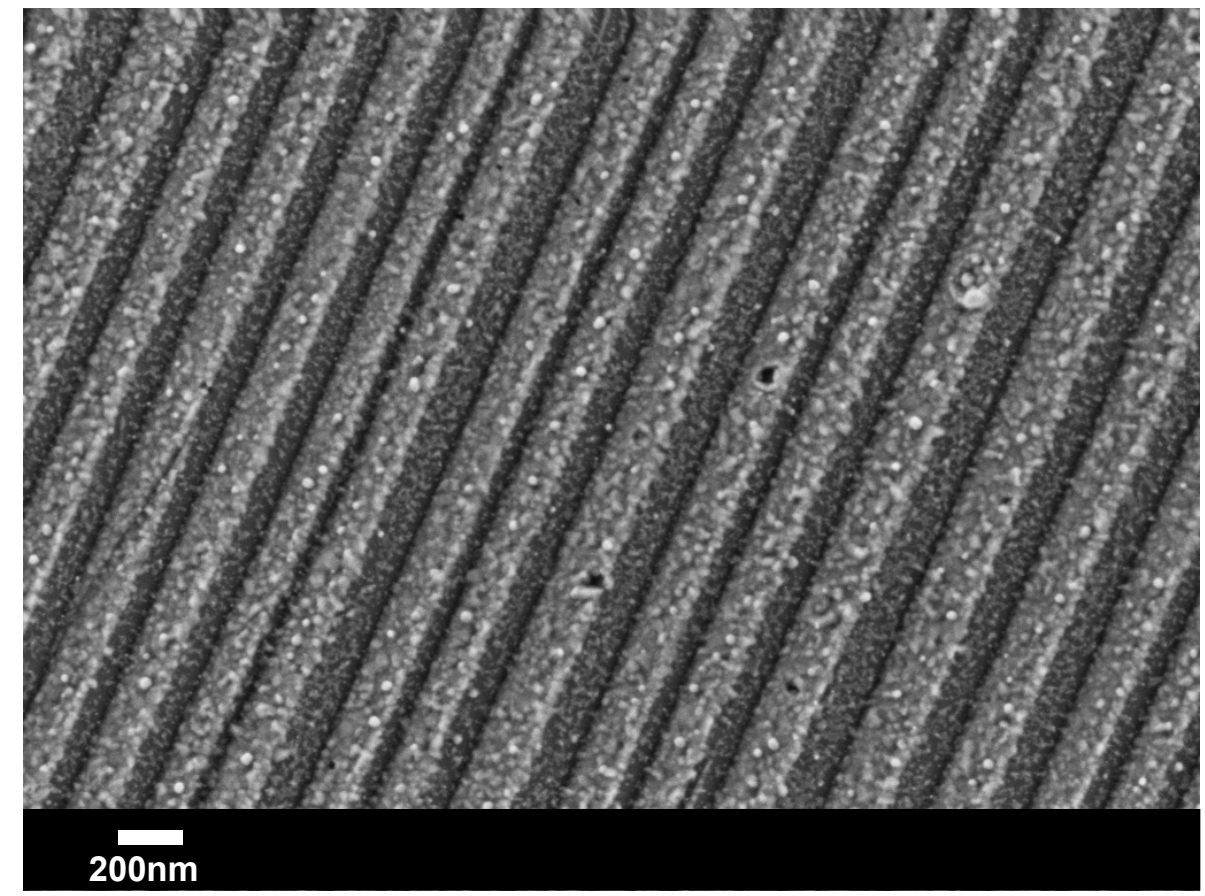

Abbildung 54: SEM-Analyse: Ca. 10nm dicke Eisendrähte nach einer Temperbehandlung für $2 \mathrm{~h}$ bei $500^{\circ} \mathrm{C}$.

Eine Steigerung der Temperungstemperatur auf $500^{\circ} \mathrm{C}$ zeigt im SEM-Bild eine Vielzahl sehr kleiner, runder Partikel auf einer sehr rauen, möglicherweise nicht zusammenhängenden Eisenschicht (Abbildung 54). Der maximal gefundene Partikeldurchmesser beträgt 60nm, die Vielzahl der Partikel ist jedoch kleiner als 25nm. Auch auf der abgeschatteten Seite sind (noch deutliche kleinere) Partikel erkennbar. 


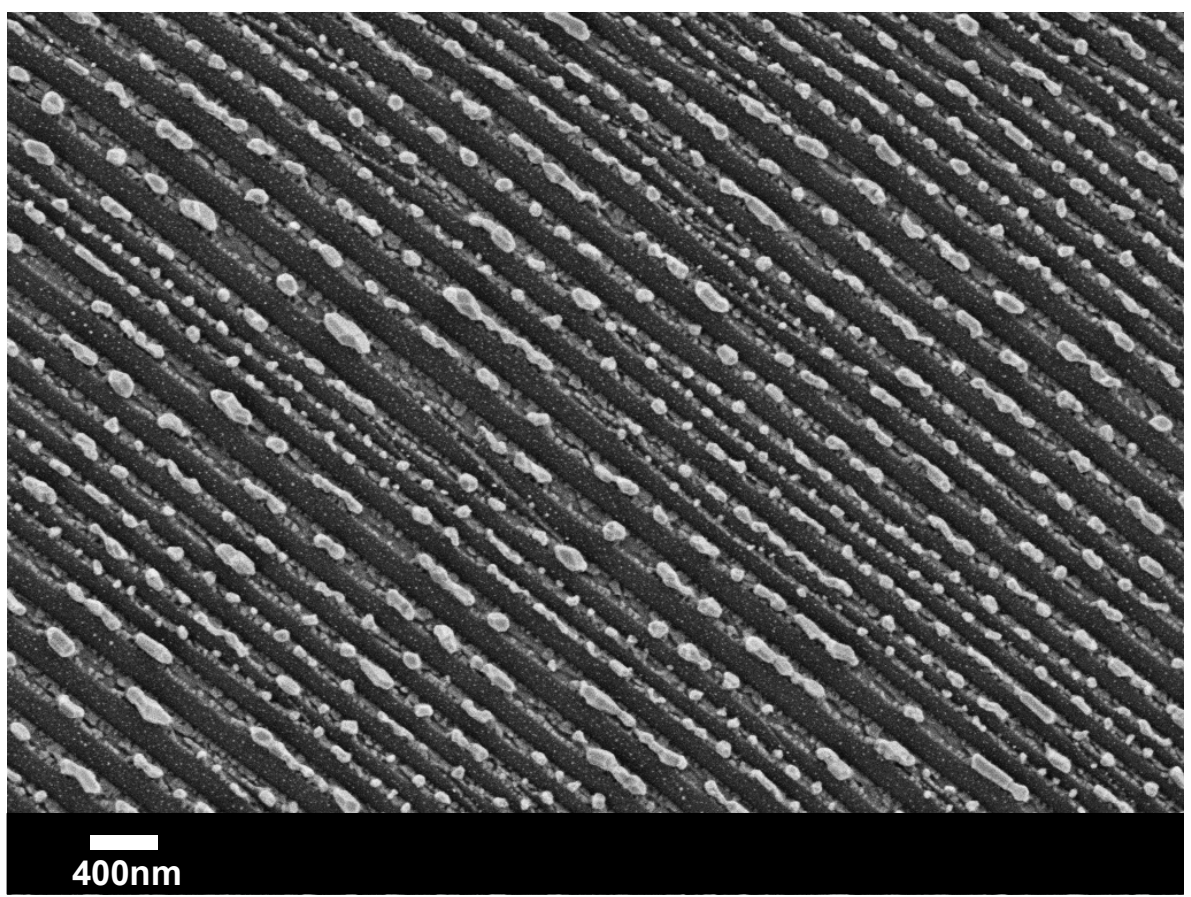

Abbildung 55: SEM-Analyse: Ca. 10nm dicke Eisendrähte nach einer Temperbehandlung für $2 \mathrm{~h}$ bei $600^{\circ} \mathrm{C}$.

Wird die Auslagerungstemperatur weiter auf $600^{\circ} \mathrm{C}$ erhöht, so wachsen große Partikel auf Kosten kleiner (Ostwald-Reifung) und es bildet sich eine perlenkettenartige Aufreihung von Partikeln entlang der Facettenkämme aus (Abbildung 55). Es ist eine klare Korrelation von Facettenbreite und Partikelgröße erkennbar, in dem Sinne, dass breitere Drähte zu größeren, aber auch weiter separierten Partikeln führen. Während kleinere Partikel bis zum Durchmesser der ursprünglichen Drahtbreite weitgehend in der Substratebene rund sind, bildet sich bei größeren Partikeln eine elliptische Vegrößerung in Richtung der Facetten aus (große Halbachse bis zu 350nm). Das Eisen ist offensichtlich nicht vollständig zu Partikeln agglomeriert, denn auf der Facettenseite, die der Quelle zugewandt ist, ist ein von der abgewandten Seite unterschiedlicher Kontrast erkennbar. Die Restschicht ist nicht zusammenhängend und durch viele Spalte unterbrochen. Auf der abgeschatteten Seite findet sich eine große Anzahl sehr kleiner Partikel mit einer mittleren Separationsdistanz von $26 \pm 5 \mathrm{~nm}$. Der Durchmesser dieser runden Partikel kann mit kleiner (bis deutlich kleiner) als 10nm abgeschätzt werden.

In Übereinstimmung mit den Leitfähigkeitsmessungen erweisen sich die bei $600^{\circ} \mathrm{C}$ ausgelagerten Drahtstrukturen anhand der SEM-Untersuchungen als in lokal sogar äquidistante Partikel zersetzt, während bei $400^{\circ} \mathrm{C}$ und $500^{\circ} \mathrm{C}$ steigende Rauigkeit als Frühstadium der Gestaltumwandlung erkennbar waren. 


\subsubsection{Lokales und globales Magnetisierungsverhalten von linear angeordneten Eisenpartikeln}

\section{Magnetisierung parallel zur Facettierungsrichtung}

Abbildung 56 zeigt Höhen- und Phaseninformation der MFM-Analyse einer bei $600^{\circ} \mathrm{C}$ getemperten Probe (vgl. Abbildung 55), die zuvor mittels eines 2T-Magneten in Facettierungsrichtung aufmagnetisiert wurde. Gut zu erkennen ist der Kontrastwechsel in Richtung der Facetten im Phasenbild (b) bei jedem (größeren) Eisenpartikel. Diese Streufeldabbildung zeigt eindeutig, dass die Teilchen parallel zur Facettierungsrichtung remanent magnetisiert sind. Durch Drehen der Abtastrichtung wurde verifiziert, dass es sich hier nicht um ein Abbildungsartefakt handelt und die Richtung, in der der Kontrast wechselt, dabei parallel zu den Facetten bleibt. Dieser

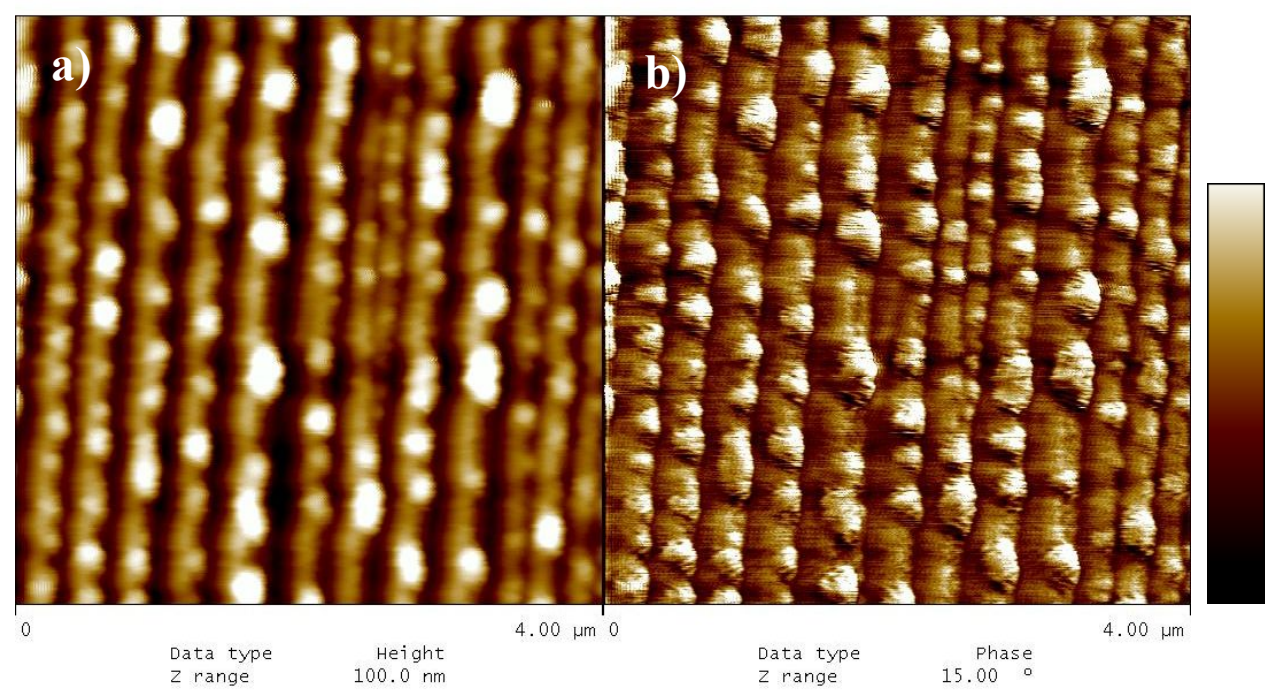

Abbildung 56: a) MFM-Höhen- und b) Phasensignal von ca. 10nm dicken Eisendrähten ausgelagert bei $600^{\circ} \mathrm{C}$, in Facettierungsrichtung mit einem 2T-Magneten magnetisiert.

Kontrastwechsel ist bei allen Partikeln gleich orientiert, d.h. ein heller Kontrast ist in Abbildung 56 im oberen, ein dunkler im unteren Teil des jeweiligen Partikels erkennbar. Oftmals ist der Abstand zwischen zwei Partikeln auf benachbarten Facetten durchaus vergleichbar zu der Distanz benachbarter Partikel auf einer Facette. Die Reste der Eisen-Drähte auf der besputterten Facettenseite sind möglicherweise für eine Vermittlung einer bevorzugten Ausrichtung der Magnetisierung der Partikel in Facettierungsrichtung verantwortlich.

Abbildung 57 zeigt dieselbe Probe wie Abbildung 56, jedoch nicht direkt nach dem Aufmagnetisieren, sondern nach 24 Stunden. Das Phasenbild zeigt nun insgesamt einen schwächeren Kontrast. Einige Partikel zeigen einen Kontrastgradienten senkrecht zur Facettierungsrichtung, d.h. die Magnetisierung der Eisenpartikel relaxiert langsam in einen Zustand, bei dem eine Magnetisierung senkrecht zur Facettierungsrichtung erreicht wird. Dies tritt bevorzugt bei elliptisch in diese Richtung verformten Partikeln auf, weshalb die Ursache in diesem System in der Formanisotropie und nicht in der magnetokristallinen Anisotropie zu sehen ist. 


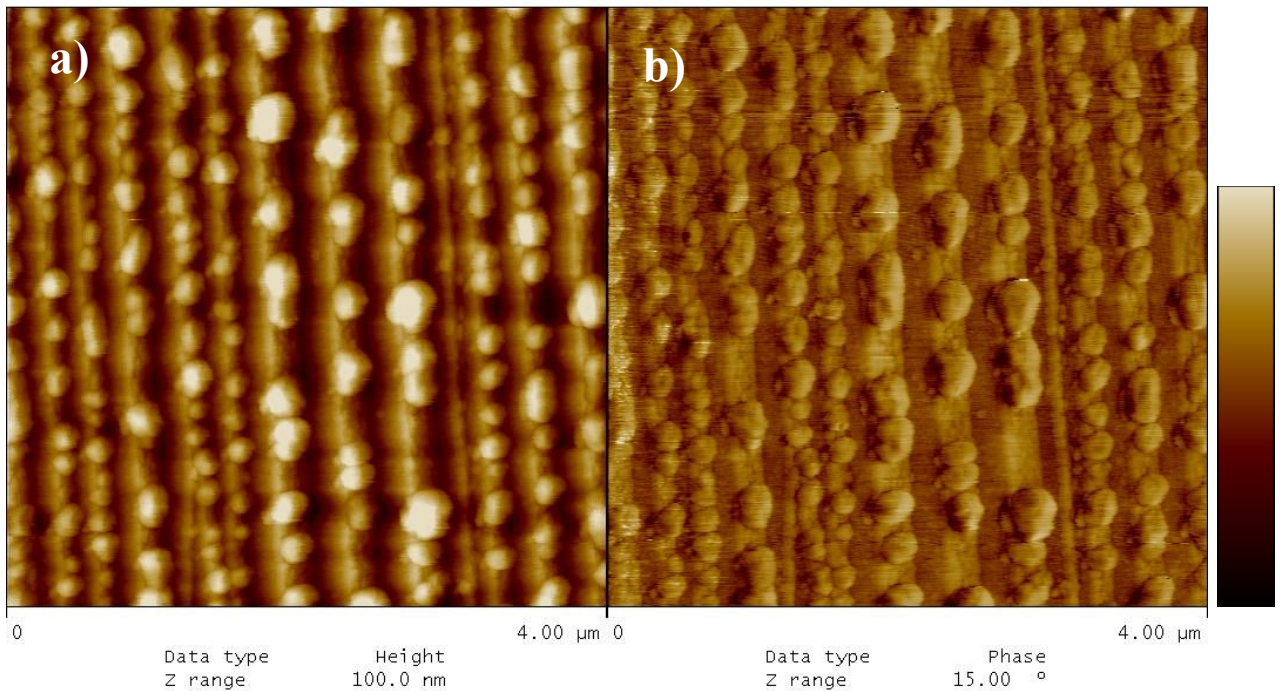

Abbildung 57: a) MFM-Höhen- und b) Phasensignal von ca. 10nm dicken Eisendrähten ausgelagert bei $600^{\circ} \mathrm{C}$, in Facettierungsrichtung mit einem 2T-Magneten magnetisiert, remanenter Zustand nach $24 \mathrm{~h}$.

\section{Magnetisierung parallel zur Substratnormalen}

Durch Anlegen eines vertikalen Magnetfeldes (parallel zur Substratnormalen) kann ein anderer remanenter Zustand beobachtet werden. In der Phasenkontrastdarstellung (Abbildung 58b) zeigen

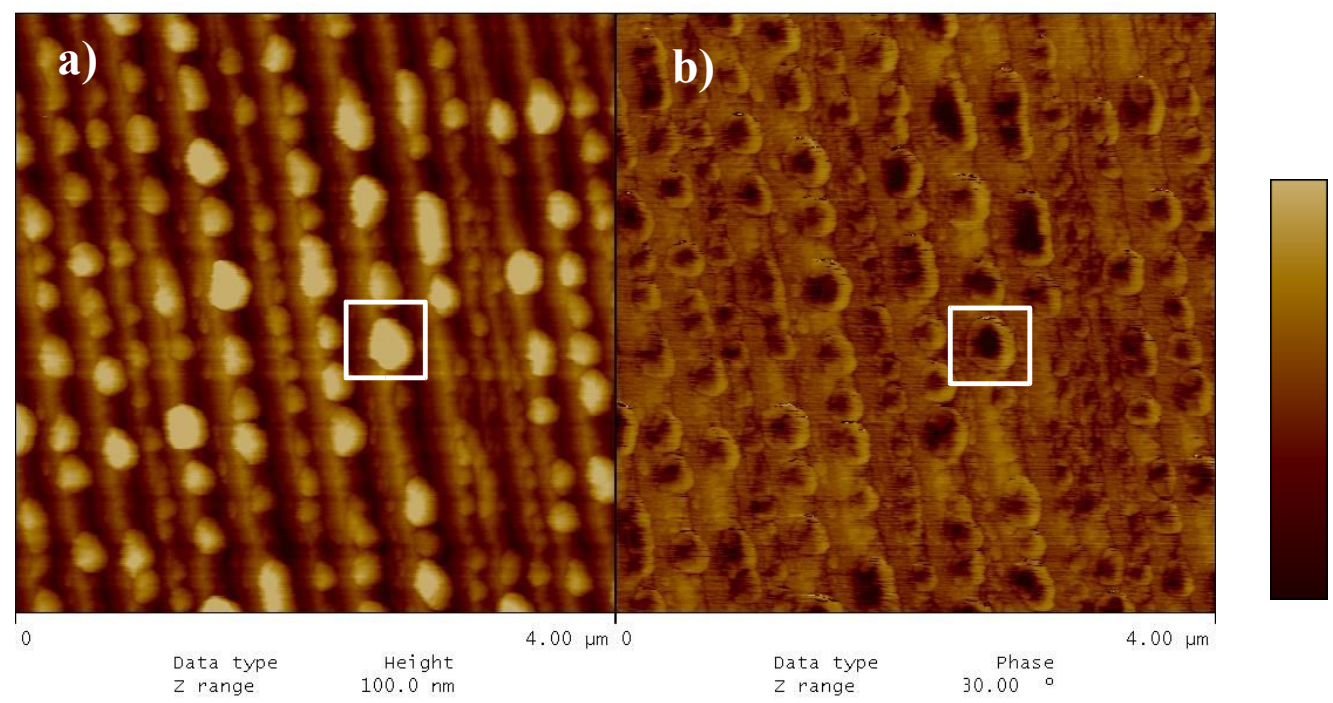

Abbildung 58: a) MFM-Höhen- und b) Phasensignal von ca. 10nm dicken Eisendrähten ausgelagert bei $600^{\circ} \mathrm{C}$, parallel zu Substratnormalen mit einem 2T-Magneten magnetisiert.

die Eisenpartikel eine typische Ringstruktur, d.h. während am Rand des Partikels eine attraktive Wechselwirkung mit der ferromagnetischen Spitze festzustellen ist, zeigt sich im Zentrum eine abstoßende Wechselwirkung.

Zum detaillierten Vergleich von Form und magnetischem Signal eines einzelnen Teilchens ist in Abbildung 59 jeweils der in Abbildung 58 eingerahmte Bereich vergrößert gezeigt. In der Flächendarstellung dieser Daten des einzelnen Eisenpartikels (Abbildung 60) wird besonders deutlich, dass sich das Eisenpartikel magnetisch in einer veränderten Form darstellt. Während die 

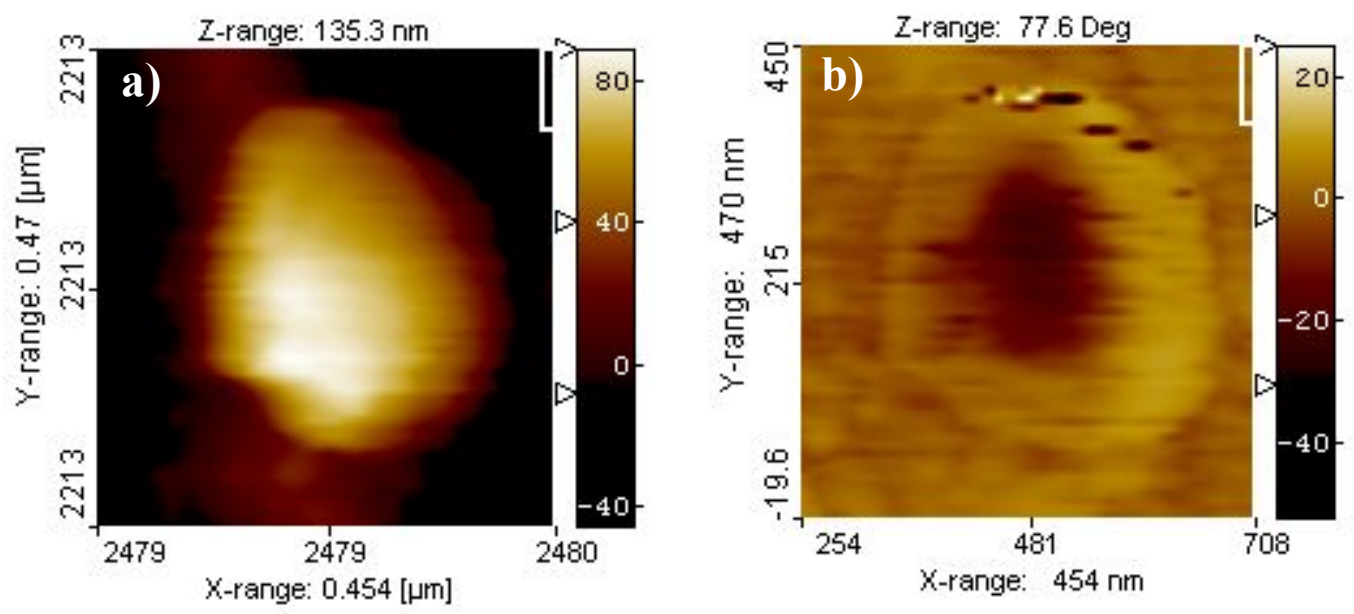

Abbildung 59: a) MFM-Höhen- und b) Phasensignal des in Abbildung 58 markierten Fe-Partikels.

Gestalt des Teilchens rund ist, ergibt die Darstellung des magnetischen Signals eine Kombination aus einem Kegel im Zentrum des Teilchens und einem Ring umgekehrten Vorzeichens um diesen Kegel. Dieser Verlauf zeigt eine vertikale Magnetisierungsausrichtung im Teilchen an, wobei der Verlauf entweder parallel oder aber antiparallel zur Magnetisierungsrichtung der Spitze ist. (Die Höhenskala in Abbildung 60b wurde zur besseren Vergleichbarkeit mit Abbildung 60a umgekehrt dargestellt.)
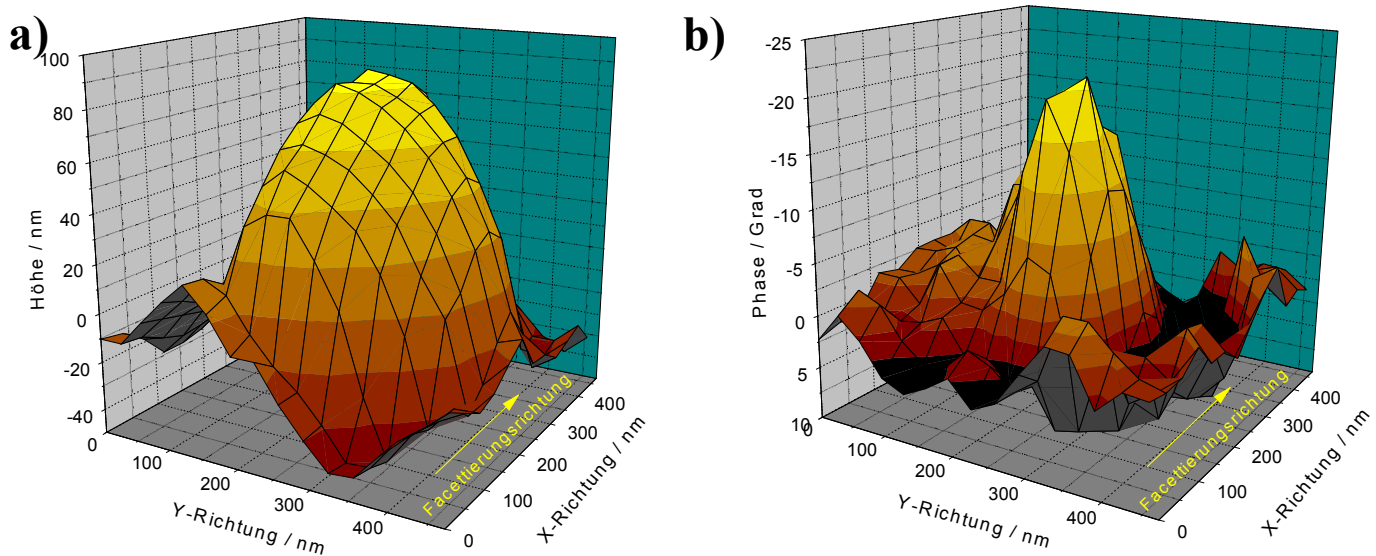

Abbildung 60: Flächendarstellung von a) Höhen- und b) Phaseninformation des Fe-Partikels aus Abbildung 59. 


\section{Magnetisierungsverhalten des Ensembles}

Während die Untersuchungen mittels Rasterelektronenmikroskopie und AFM bzw. MFM nicht vollständig klären konnten, ob nach einer $4 \mathrm{~h}$ Temperung bei $500^{\circ} \mathrm{C}$ noch raue, durchgehende Drähte vorliegen oder aber bereits separierte Partikel, liefert die Analyse des Magnetisierungsverhaltens mittels MOKE hier weitere Hinweise.

Die gemessenen Hysteresekurven für Eisendrähte nach einer Temperung für $4 \mathrm{~h}$ bei $500^{\circ} \mathrm{C}$ zeigt Abbildung 61. Bei deutlich reduziertem Signal ist bei jeder Orientierung bezüglich des äußeren Magnetfeldes die Sättigung erreicht. Ein Vergleich mit dem Magnetisierungsverhalten der wie hergestellten Drähte (Abbildung 40) macht die starke Veränderung besonders deutlich. Während das Magnetisierungsverhalten im wie hergestellten Fall sehr stark von der Orientierung der Nanodrähte bezüglich des Feldes abhängig war, liegt nach der Temperung bei $500^{\circ} \mathrm{C}$ über den gesamten
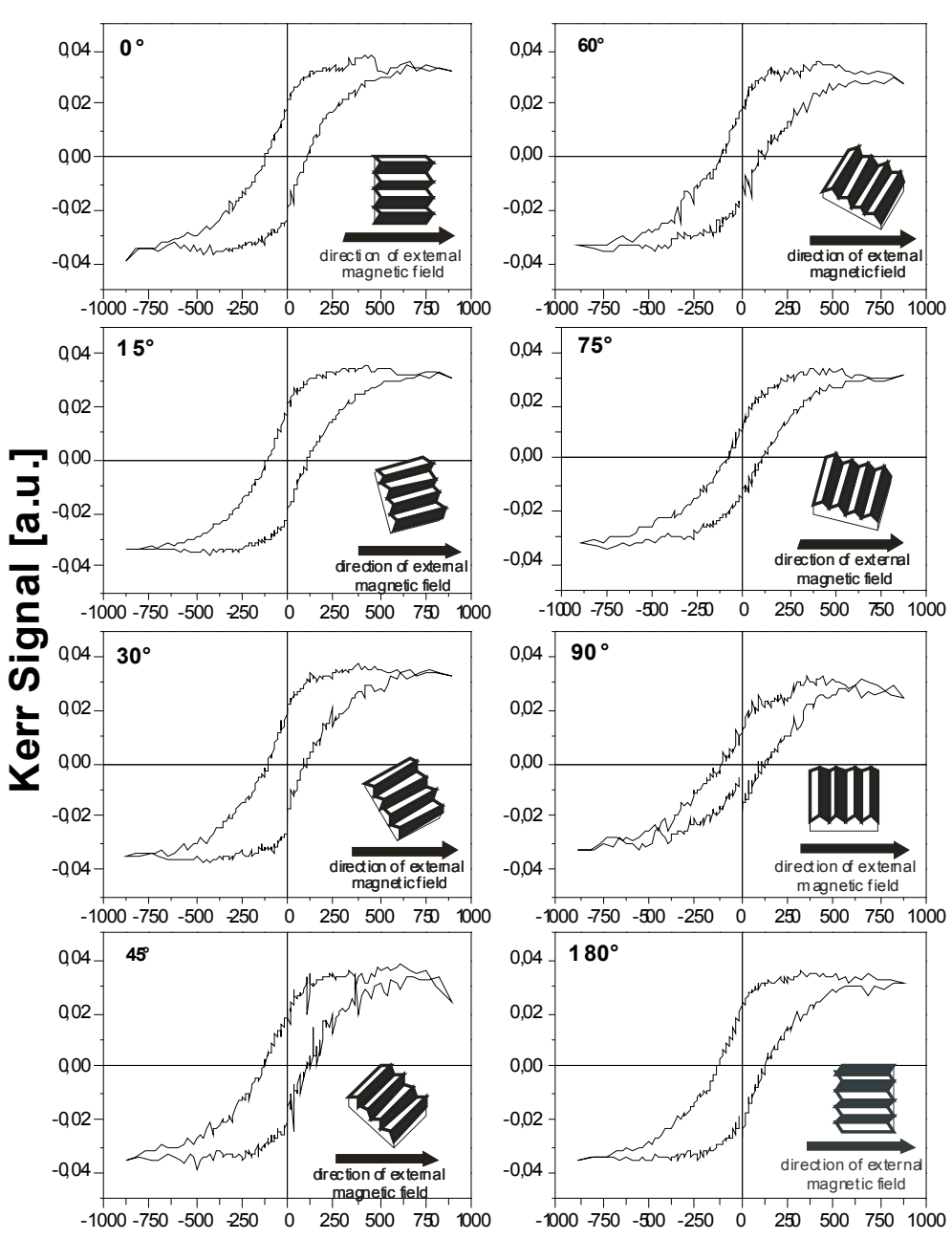

\section{$\mathrm{H}$ [Oe]}

Abbildung 61: Magnetisierungsverhalten mittels MOKE für ca. 10nm dicke $\mathrm{Fe}$-Drähte nach Tempern für $4 \mathrm{~h}$ bei $500^{\circ} \mathrm{C}$.

Winkelbereich eine weitgehend isotrope ferromagnetische Antwort vor. Die Morphologie des Substrats und die einseitig beschichteten Facetten haben keinen Einfluss mehr auf das magnetische Verhalten der Probe. Offensichtlich liegen die ferromagnetischen Bereiche in einer, auf die Facettenseite bezogen, runden Form vor, durch die keine Richtung in dieser Ebene durch Formanisotropie bevorzugt wird.

Besonders deutlich machen dies die Abbildungen 62 und 63, in denen die Koerzitivfeldstärke und das Verhältnis $\mathrm{M}_{\mathrm{r}} / \mathrm{M}_{\mathrm{s}}$ für die wie hergestellte und die ausgelagerte Probe gegen den Winkel zwischen äußerem Feld und Facettierungsrichtung aufgetragen ist. Beide Größen sinken mit der Auslagerung und die Winkelabhängigkeit wird geringer.

Es ist bekannt, dass das Koerzitivfeld von aufgedampften Eisenfilmen durch Oxidation steigt [Tak 83]. Da die Temperung der Schichten bei einem Restgasdruck besser 1,910 $10^{-7} \mathrm{mbar}$ erfolgte und das Koerzitivfeld (wie in Abbildung 62 dargestellt) durch die Temperung sinkt, ist von einer Oxidation maßgeblicher Volumenanteile des Eisens nicht auszugehen. Neben der strukturellen Entkopplung der Eisenpartikel bei der Desintegration der Drähte kann eine geringfügige Oberflächenoxidation zusätzlich zur magnetischen Entkopplung der Eisenpartikel führen [Bia 02]. 


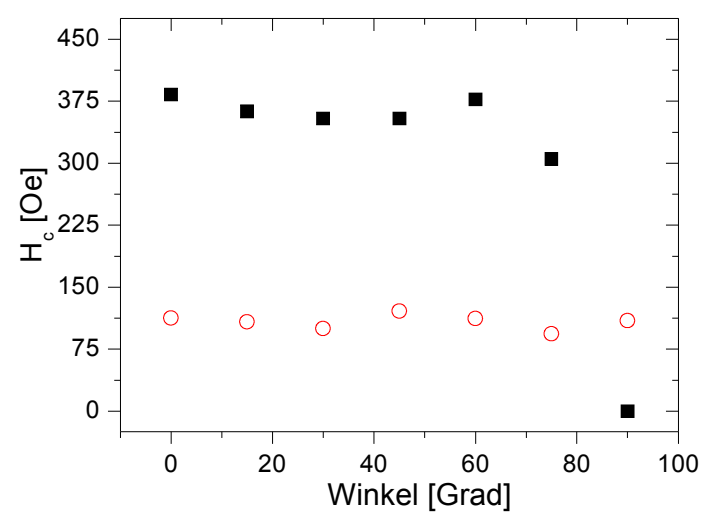

Abbildung 62: Koerzitivfeld der ca. 10nm dicken FeDrähte in Abhängigkeit vom Winkel zwischen Facettierungsrichtung und äußerem Magnetfeld. Schwarze, volle Quadrate wie hergestellt; rote Kreise nach dem Tempern für $4 \mathrm{~h}$ bei $500^{\circ} \mathrm{C}$.

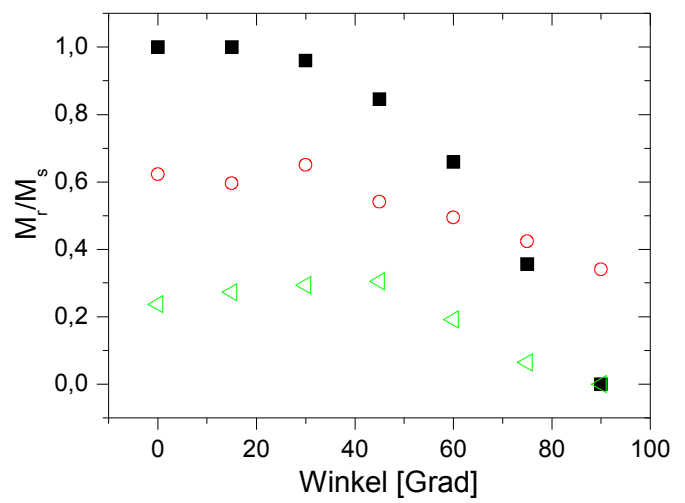

Abbildung 63: $M_{r} / M_{s}$ der ca. 10nm dicken Fe-Drähte in Abhängigkeit vom Winkel zwischen Facettierungsrichtung und äußerem Magnetfeld. Schwarze, volle Quadrate wie hergestellt; rote Kreise $4 \mathrm{~h}$ bei $500^{\circ} \mathrm{C}$; grüne Dreiecke $2 \mathrm{~h}$ bei $600^{\circ} \mathrm{C}$.

Tatsächlich konnten Mentz et al. bei Bedeckungen in der Nähe der Perkolationsschwelle zeigen, dass eine langreichweitige ferromagnetische Ordnung mit Verbreiterung der Hysterese und somit Zunahme des Koerzitivfeldes erst bei Zusammenwachsen von ferromagnetischen Eiseninseln (aus zwei Atomlagen) zu beobachten ist [Men 99]. Sugawara et al. zeigten, dass lineare Anordnungen von polykristallinen Eiseninseln der Größe von 5,1 $\pm 2,8 \mathrm{~nm}$ bis $6,7 \pm 2,3 \mathrm{~nm}$, einer Höhe von 0,6nm bis $1,0 \mathrm{~nm}$ und einer Separierung von $9 \mathrm{~nm}$ bei Raumtemperatur vermittelt durch die magnetostatische Dipolkopplung zwischen den Inseln ferromagnetisch ordnen. Dabei finden sie eine leichte Richtung bei MOKE-Experimenten entlang der Inselketten und eine magnetisch schwere Richtung senkrecht zu diesen [Sug 97a].

Die Interpretation der SEM-Untersuchungen an den $4 \mathrm{~h}$ bei $500^{\circ} \mathrm{C}$ getemperten Drähten, nämlich dass diese in viele kleine Körner zerfallen sind, wird also durch die winkelabhängigen Messungen zur Gesamtmagnetisierung bestätigt. Auf einer Facettenseitenbreite liegen mehrere Partikel nebeneinander vor und deren Größe kann aus den SEM-Bildern auf $<50 \mathrm{~nm}$ abgeschätzt werden. Wahrscheinlich liegt aber eine nicht verschwindende Anzahl von Partikeln in einer Größe vor, die jenseits des superparamagnetischen Limits bei Raumtemperatur ist. 5-8nm werden im Bereich der Dünnschichttechnologie in der Literatur als mittlere magnetische Bereichsgröße genannt, unterhalb der keine feste Magnetisierungseinstellung mehr erfolgen kann und das Moment thermisch aktiviert fluktuiert [Sug 97a]. Dies schließt aber nicht aus, dass sehr dünne, noch zusammenhängende Bereiche einen Einfluss auf die Magnetisierungskurven haben. So würde eine Unterschreitung der kritischen Filmdicke für den Spinreorientierungsübergang zu einer Magnetisierung parallel zur Substrat- bzw. Facettenseitennormalen in diesen Bereichen führen, wenn dort eine vom Rest unabhängige Ausrichtung möglich ist. Mentz et. al [Men 99] und Meyer [Mey 03] konnten zeigen, dass eine Spinreorientierung im System von wenigen Monolagen Eisen auf $\mathrm{Cu}(100)$ zu finden ist. Die Filmdicke, bei der ein Spinreorientierungsübergang im System Eisen auf $\mathrm{Cu}(100)$ erfolgt, steigt mit zunehmender Auslagerungstemperatur an. So zeigen bei $100 \mathrm{~K}$ hergestellte Eisen-Filme einen Anstieg der Übergangsdicke von 3,8ML auf 6-6,5ML bei Temperungen bis zu 420K [Men 97]. Ob tatsächlich ein Spinreorientierungsübergang die Hysterese beeinflusst, kann nicht abschließend beantwortet werden. Es liegen jedoch sicher Bereiche sehr unterschiedlicher Eisendicke durch Abschattung, aber auch den Eintrag geringer Mengen Eisens auf die abgeschattete Seite von nahezu thermalisierten und somit ungerichteten Atomen vor. Somit ist ein entsprechender Einfluss aus dünnen Schichtbereichen durchaus möglich. Als wahrscheinlicher ist aber anzusehen, dass eine verbleibende magnetokristalline Anisotropie in der Substratebene vorliegt. 
Aus den Ergebnissen der vorliegenden Untersuchungen lässt sich folgern, dass die Reduktion im Signal (proportional zur Gesamtmagnetisierung) und das reduzierte Koerzitivfeld bei der Temperung bei $500^{\circ} \mathrm{C}$ auf erste Unterbrechungen in den Eisendrähten, die die langreichweitige ferromagnetische Ordnung in benachbarten Drahtbereichen behindern, zurückzuführen ist.

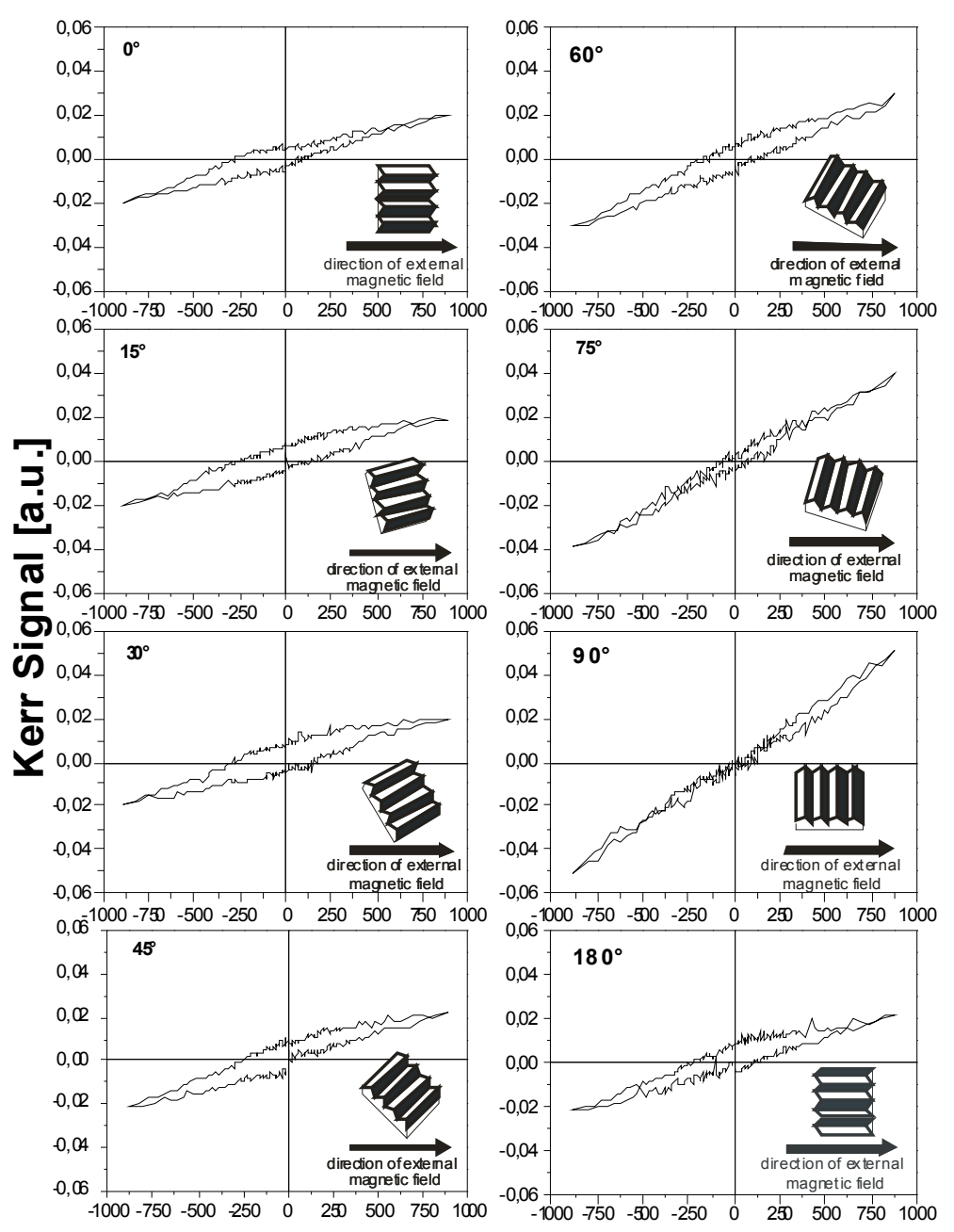

$\mathrm{H}[\mathrm{Oe}]$

Abbildung 64: Magnetisierungsverhalten mittels MOKE für ca. 10nm dicke Fe-Drähte nach Tempern für $2 \mathrm{~h}$ bei $600^{\circ} \mathrm{C}$.
Eine lokale magnetostatische Dipolkopplung benachbarter Partikel kann nicht vollständig ausgeschlossen werden, sicher ist aber, dass die Anordnung der Partikel in Form der vormaligen Drähte, d.h. die extremen Aspektunterschiede zwischen Breite und Länge der Anordnung, keinen Einfluss auf das Magnetisierungsverhalten hat.Nach einem weiteren Temperschritt für $2 \mathrm{~h}$ bei $600^{\circ} \mathrm{C}$ bei sonst gleichen Bedingungen ändert sich das Magnetisierungsverhalten erneut (siehe Abbildung 64). War nach der Temperung bei $500^{\circ} \mathrm{C}$ das zur Verfügung stehende maximale äußere Feld von 8500e noch ausreichend, um eine Sättigung herbeizuführen, so zeigt die Probe nach der weiteren Wärmebehandlung deutlich höhere Sättigungsfelder. Zwar folgt die Magnetisierung dem äußeren Feld, aber ein Einmünden in eine Sättigungsmagnetisierung ist bei keiner Winkelanordnung zwischen äußerem Feld und Facettierungsrichtung $\mathrm{zu}$ erkennen.

Daher werden in einem Feld bis 850Oe keine vollständigen Hysteresen durchfahren (siehe z.B. [Sor 03]) und eine quantitative Auswertung wird damit erschwert.

Die Kurven zeigen nur eine kleine Öffnung, wobei sowohl Koerzitivfeld als auch Remanenzmagnetisierung klein sind. Eine Vergrößerung des Winkels zwischen äußerem Feld und Facettierungsrichtung hat bis zum Winkel von $90^{\circ}$, bei dem der Verlauf der vormaligen Drähte gerade senkrecht zum äußeren Feld ist, einen Anstieg der Steigung der Magnetisierungsverläufe zur Folge. Dies kann dahingehend interpretiert werden, dass die Öffnung der gesamten Hysteresekurve im parallelen Fall am größten ist und bei Annäherung an $90^{\circ}$ kleiner wird.

Elmers et al. konnten durch Experimente (u.a. bei 300K) mit variierendem Bedeckungsgrad $\theta$ von Eisen auf Wo(110) zeigen, dass die ferromagnetische Ordnungseinstellung trotz möglicher Dipol- 
Kopplung nur um $\theta=0,02$ vor dem Zusammenwachsen der Eiseninseln bei $\theta=0,6$ erfolgt [Elm 94]. Für die Interpretation der vorliegenden Ergebnisse heißt das, dass schon eine sehr geringe Separation von Eisenpartikeln durch den Temperprozess zu einem Verschwinden der ferromagnetischen Ordnung führt. Somit kann der Einbruch in der Signalstärke der MOKEUntersuchungen schon nach dem Tempern bei $500^{\circ} \mathrm{C}$ durch den Zerfall des überwiegenden Materialanteils in kleine, (magnetisch) isolierte Eisenpartikel erklärt werden. Dabei kann sowohl eine räumliche Distanz als auch eine geringe Oberflächenoxidation zur Separation beitragen.

Eine Zusammenfassung der Ergebnisse dieses Kapitels ergibt, dass sich separierte Partikel, abhängig von ihrer Gestalt durch ein äußeres Feld in dessen Richtung magnetisieren lassen. Dies konnte sowohl in der Substratebene, wie auch senkrecht dazu nachgewiesen werden. Elliptische Partikel zeigen eine Relaxationen der Magnetisierung in Richtungen der langen Halbachse. Eine winkelabhängige Betrachtung der Gesamtmagnetisierung bestätigt einen Zerfall der Drähte in weitgehend separierte Partikel bei einer Temperung $\mathrm{ab} 500^{\circ} \mathrm{C}$.

\subsubsection{Einfluss von Materialmenge und -verteilung am Beispiel Kobalt}

Am Beispiel Kobalt wurde untersucht, ob eine dünne Einzelschicht unter vergleichbaren Bedingungen, unter denen eine Gestaltumwandlung von Drähten zu separierten Partikeln auftritt, eine Gestaltänderung erfährt. Ebenso wurde der Einfluss der unterschiedlichen Drahtdicke von $\sim 5 \mathrm{~nm}$ und $\sim 20 \mathrm{~nm}$ auf die Gestaltänderung und die resultierende Partikelanordnung untersucht.

Abbildung 65 zeigt MFM-Analysen der ca. 5nm dicken Kobalt-Einzelschicht nach Auslagerung für zwei Stunden bei $550^{\circ} \mathrm{C}$. Während die Topologie (a) durch das Tempern weitgehend unverändert bleibt, ist der Kontrast bezüglich der Domänen gegenüber dem Zustand nach der Herstellung leicht
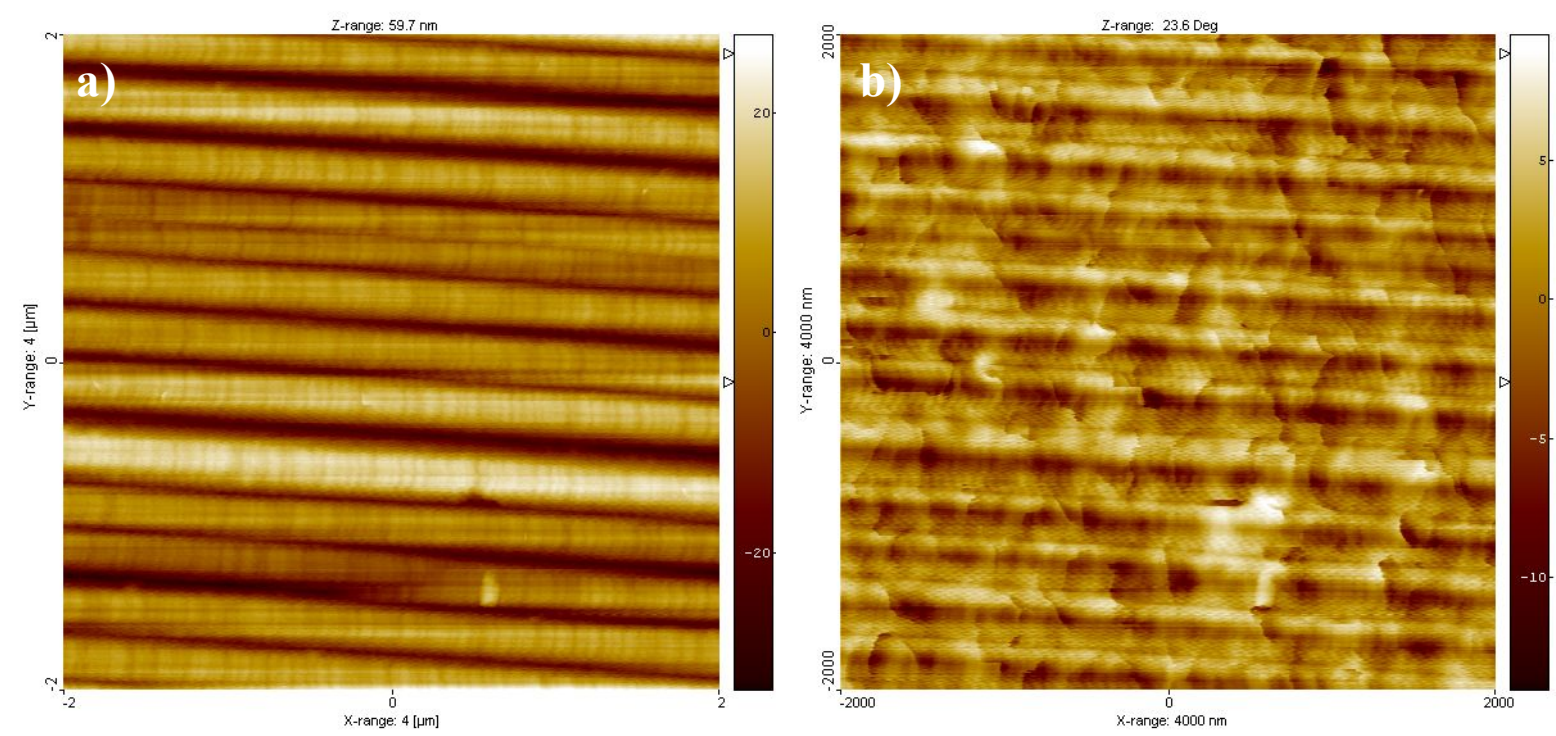

Abbildung 65: a) MFM-Höhen- und b) Phasensignal einer ca. 5nm Einzelschicht Kobalt auffacettiertem $\mathrm{Al}_{2} \mathrm{O}_{3}$ nach $2 \mathrm{~h}$ Tempern bei $550^{\circ} \mathrm{C}$; Probe nicht magnetisiert. 
vergrößert (b). Die mittlere Größe der magnetischen Domänen reduziert sich auf ca. 250-450nm in Facettierungsrichtung. Es sind aber durchaus auch wesentlich kleinere, aber auch bis $\mathrm{zu} 800 \mathrm{~nm}$ große Magnetisierungsbereiche erkennbar. Die geringen Veränderungen, sowohl im Höhen-, wie auch im magnetisch sensitiven Phasensignal zeigen, dass die dünne Kobaltschicht keine wesentliche Gestaltänderung erfährt. Ein Zerfall in Streifen oder separierte Partikel findet nicht statt. Dennoch ist es möglich, dass die starke Krümmung an den Extrempunkten der Facettenkämme und -täler zu vermehrter Defektanlagerung (z.B. Korngrenzen) in diesen Bereichen der Kobaltschicht geführt hat, wie sie bei Schichtpaketen oberhalb der Facettenkämme beobachtet werden (siehe Kapitel 5.1.5). Diese vergleichsweise geringen mikrostrukturellen Veränderungen haben nur geringen Einfluss auf die Streufeldverteilung über der Probe und damit auf das detektierbare Phasensignal.

Während die ca. 5nm dicken Kobaltdrähte mittels SEM- und AFM nur sehr schwer abzubilden waren, zeigt die MFM-Analyse nach zweistündigem Tempern, dass Kobalt präferenziell auf einer der beiden Facettenseiten abgeschieden wurde. Durch die Auslagerung haben sich weitgehend runde Partikel eines mittleren Durchmessers von 235nm gebildet (Abbildung 66a und Histogramm
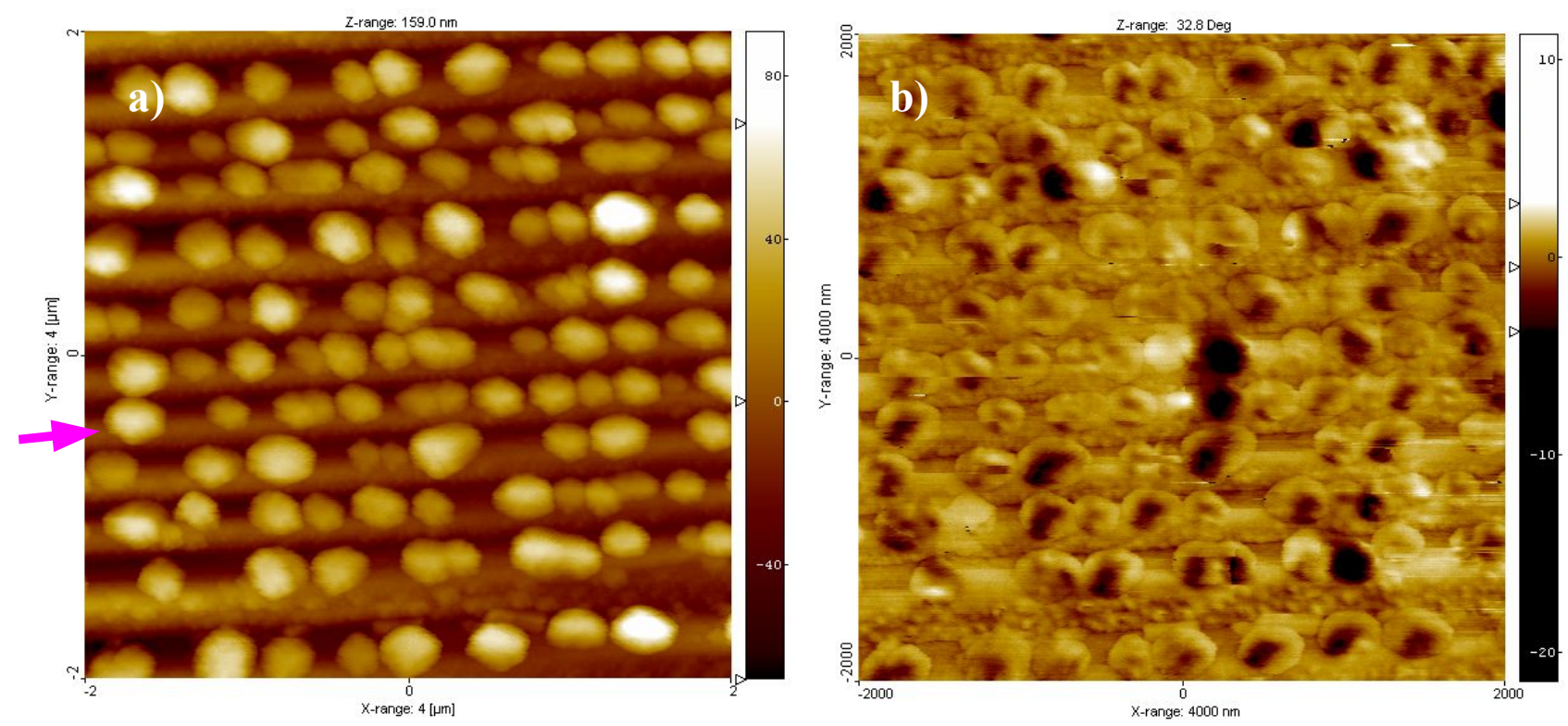

Abbildung 66: a) MFM-Höhen- und b) Phasensignal von ca. $5 \mathrm{~nm}$ Co-Drähten, $2 \mathrm{~h}$ bei $550^{\circ} \mathrm{C}$ getempert auf facettiertem $\mathrm{Al}_{2} \mathrm{O}_{3}$; Probe nicht magnetisiert.

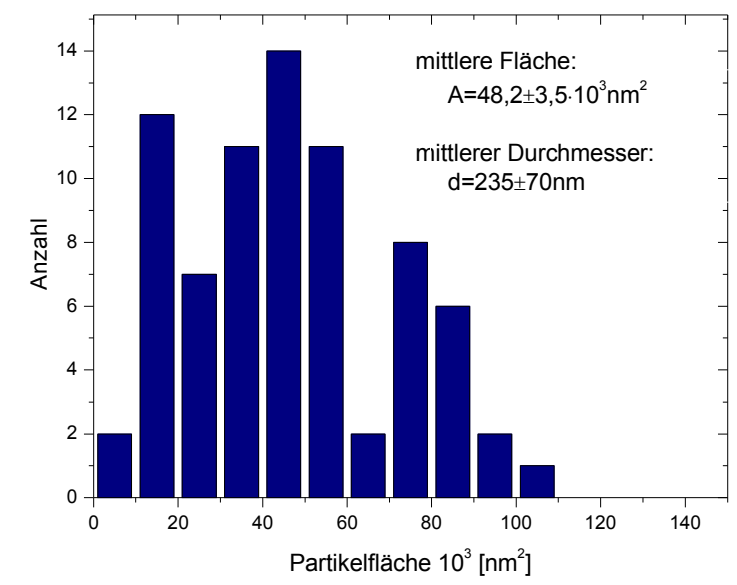

Abbildung 67: Größenverteilung der mittleren Flächen und resultierender Durchmesser eines Co-Partikels aus Abbildung 66a).
Abbildung 67), die in der Regel separiert sind, typische Höhen der Partikel über der Facettenseite betragen 40-70nm. Der Vergleich mit den unwesentlichen Veränderungen der Einzelschicht vergleichbarer Dicke (vgl. Abbildung 65) durch die Temperaturbehandlung zeigt, dass die Präformierung in Drähte (und damit die Reduzierung der Dimension) wesentlich für die Umformung in Partikel und damit für die Stabilität der Ausgangsstruktur ist. Die Partikel liegen vollständig in Reihen angeordnet vor, da sie nur auf einer der beiden Facettenseiten liegen. Dementsprechend ist ihre mittlere Separation senkrecht zur Facettierungsrichtung durch die Breite der anderen Facettenseite und die Partikel- 
größe bestimmt. Die Variation der Facettenbreite und die Breite der Verteilung des Partikeldurchmessers ergeben einen typischen Abstand der Partikel in dieser Richtung von 160-235nm. In Facettierungsrichtung hingegen ist die Separation allein durch den Zerfalls- und den Vergröberungsprozess bestimmt. Es ist ein deutlicher Zusammenhang zwischen der Separation und der Partikel größe erkennbar. Während beispielsweise auf der in Abbildung 66 mit einem magentafarbenen Pfeil gekennzeichneten Facette die annähernd gleich großen Partikel (d 260nm) nur wenige Nanometer (bis zu 30nm) in Facettierungsrichtung getrennt sind, entstehen in direkter Nachbarschaft zu sehr großen Partikeln (d 420nm), z.B. in der darunter liegenden Reihe, Distanzen zum nächsten Kobaltpartikel, die mit $430 \mathrm{~nm}$ im Bereich des Durchmessers dieser Partikel liegen.

Hieraus wird deutlich, dass nach dem Zerfall der Drähte in separierte Partikel mit kleiner
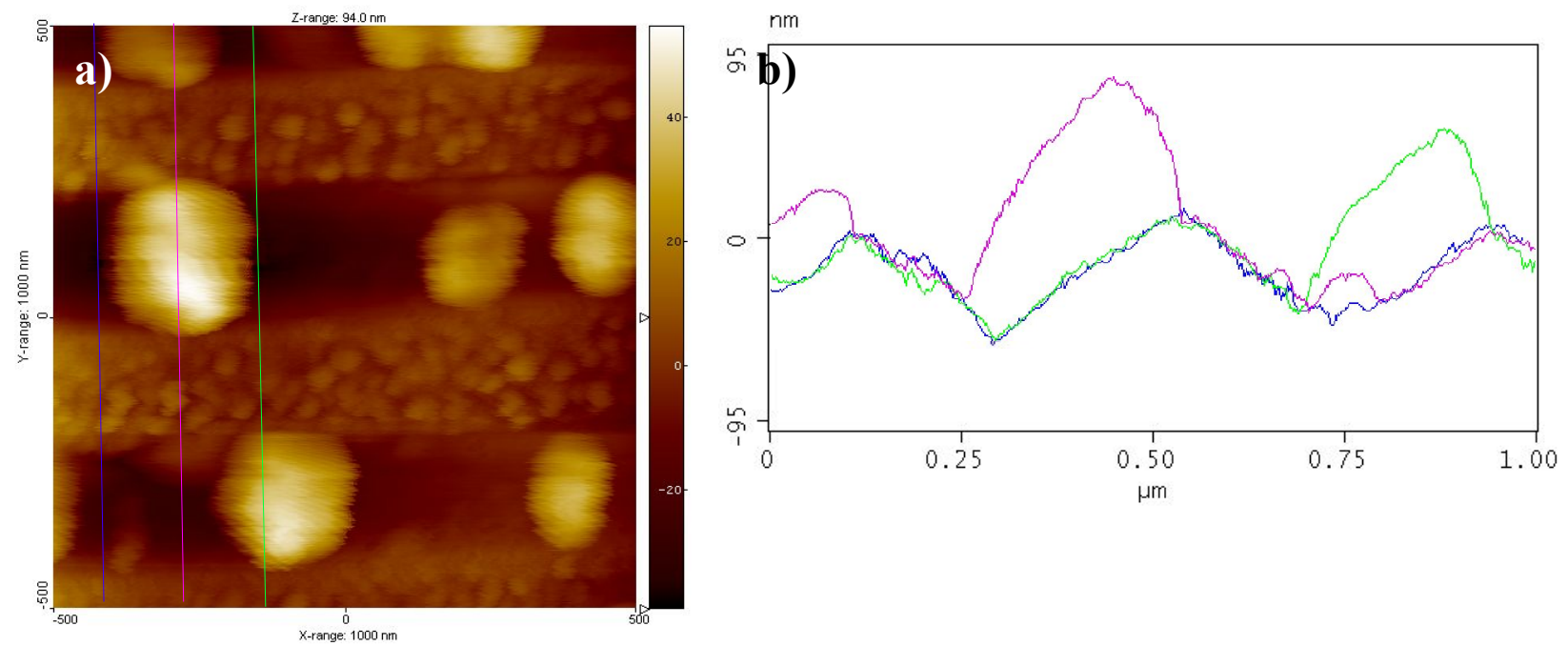

Abbildung 68: a) MFM-Höhendarstellung mit b) mehrfacher Schnittdarstellung, von ausgewählten Co-Partikeln aus ca. $5 \mathrm{~nm}$ Co-Drähten nach $2 \mathrm{~h}$ bei $550^{\circ} \mathrm{C}$; Probe nicht magnetisiert.

interpartikulärer Distanz eine Vergröberung im Sinne einer Ostwaldreifung stattfindet, d.h. große Partikel wachsen auf Kosten kleinerer. Die Unterschiede im chemischen Potential durch die verschiedenen Krümmungsradien bewirken einen Diffusionsstrom in Richtung des größeren Partikels. Als Folge davon befindet sich in der Umgebung von großen Partikeln ein Hof, da diese durch das Material der umliegenden kleineren Partikel gewachsen sind. Beim Zusammenschluss von Partikeln bilden sich Hälse zwischen diesen aus, bis schließlich durch Volumen- und Oberflächendiffusion ein größeres rundes Partikel resultiert. Die Mechanismen der Vergröberung durch Zusammenschluss und des Clusterwachstums auf Oberflächen sind komplex, eine genauere Darstellung findet sich in [Nic 66], durch neuere Monte Carlo Simulationen analysieren Carrey et al. Größen- und Zeitabhängigkeit des Zusammenschluss [Car 02].

Ein ähnlich wechselnder Kontrastverlauf in der magnetischen Phasenabbildung bei mehreren Partikeln, die in vergleichbarem Abstand auf einer Facette liegen (siehe z.B. untere Reihe in Abbildung 66b), deutet auf eine magnetostatische Kopplung zwischen diesen Partikeln hin.

Ein Bildausschnitt von nur $1 \mu \mathrm{m}^{2}$ (Abbildung 68a) zeigt weitere wichtige Details auf. Während die Bereiche zwischen den Partikeln in Facettierungsrichtung sehr glatt sind (RMS: 1,2-1,7nm auf $150 \mathrm{~nm}^{2}$ ), zeigt die andere Facettenseite eine deutlich höhere Rauigkeit (RMS 4,1-5,9nm auf $150 \mathrm{~nm}^{2}$ ). Es sind flache, dicht aneinander gelegene Erhöhungen eines Durchmessers von 30-50nm (Höhe $<5 \mathrm{~nm}$ ) sichtbar. Kobalt, das sich bei der Deposition auf der abgeschatteten Facettenseite niedergeschlagen hat, ist wahrscheinlich Ursache dieser Partikel.

In Abbildung 68b sind farbig codiert Schnitte senkrecht zur Facettierungsrichtung vor, auf und hinter einem Partikel dargestellt. Die Zusammenstellung zeigt sehr gut Lage und Form in dieser Schnittrichtung. An der höchsten Stelle überragt das Partikel die Facettenspitze um $69 \mathrm{~nm}$ und ist 
damit mehr als doppelt so hoch wie die Facette. Dennoch zeigt die Darstellung in Abbildung 68 und die zugehörende dreidimensionale Darstellung

(Abbildung 69), dass die Partikel stark auf den einen Facettenseitentyp begrenzt sind. Insbesondere über die Facettenspitze hinweg findet keine Ausdehnung statt. Vielmehr findet aber auch bei der Temperung kein Materialtransport über die Facettenkämme hinweg statt. Die Rauigkeit durch das Material auf der abgeschatteten Seite ist in der

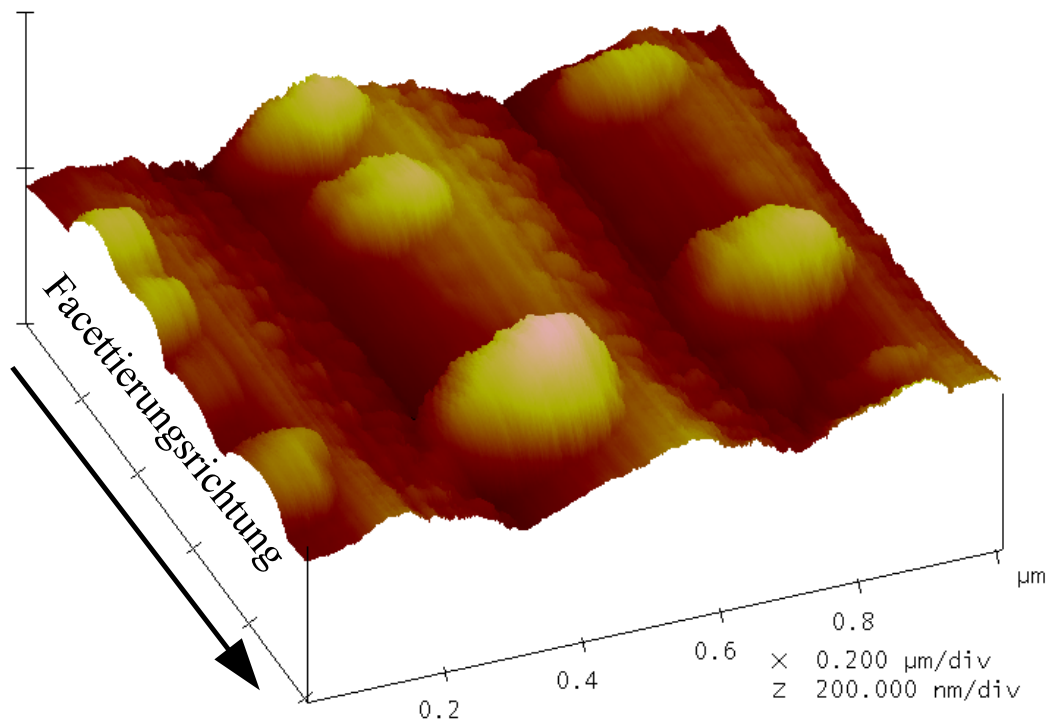

Abbildung 69: MFM-Höhendarstellung ausgewählter Co-Partikel aus ca. $5 \mathrm{~nm}$ Co-Drähten nach $2 \mathrm{~h}$ bei $550^{\circ} \mathrm{C}$; Probe nicht magnetisiert. Umgebung um die Partikel auf der anderen Facettenseite unverändert. Dies gilt sowohl für die Umgebung in Kamm-, wie auch in Talnähe, d.h. die Extrema der Facettierung behindern den Transport des Kobalts auf der Oberfläche.

Die Verteilung und Anzahl der Partikel wird auch von der Zahl an Nukleationszentren beeinflusst. Während auf der glatten (10 11$)$-Kristallfläche, der Drahtseite, gute Bedingungen für einen Transport über weite Distanzen existieren, wirken die Nanofacetten auf der abgeschatteten Seite als Keimbildungszentren für die Bildung von Kobaltpartikeln beim Zerfall der dünnen Bedeckungs schicht. Daher finden sich auf dieser Seite eine größere, teilweise in Reihen geordnete Anzahl von

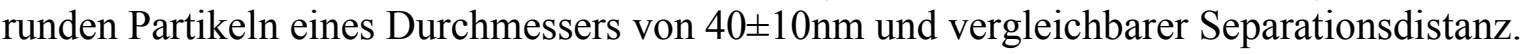

Separierte Kobaltpartikel oder kobalthaltige Legierungen sind Gegenstand intensiver aktueller Forschung mit dem Ziel der Datenspeicherung mit extrem hoher Informationsdichte. Daher ist in diesem Fall die aus dem magnetisch sensitiven Phasensignal zu ermittelnde lokale Magnetisierung der Partikel von besonderem Interesse. Die Übersichtsabbildung $66 \mathrm{~b}$ zeigt verschiedene magnetische Eigenschaften unterschiedlicher Partikel. Auffallend ist, dass insbesondere die Partikel, die in Facettierungsrichtung ausgedehnt sind, d.h. von der runden Form in eine elliptische übergehen, einen starken Kontrastwechsel im Phasensignal zeigen. Abbildung 70 zeigt eine dreidimensionale Darstellung des Höhen- und Phasensignals von zwei Teilchen, eines mittig in der Abbildung, mit starker Elongation, das zweite, runde am oberen Bildrand.

Zunächst zum Kontrast des elongierten Partikels. Das Phasenbild zeigt jeweils ein deutlich ausgeprägtes Maximum bzw. Minimum an den Enden des Partikels. Dies entspricht einer Magnetisierung in der Substratebene in Richtung der langen Achse des Partikels. Dabei zeigt eine genaue Analyse, dass die Achse zwischen Minimum und Maximum gegen die Facettierungsrichtung leicht gedreht ist, wofür wahrscheinlich die magnetokristalline Anisotropie (Kristallanisotropie) ursächlich ist.

Das runde Partikel hingegen zeigt einen ähnlichen Phasenkontrast, wie er schon bei der Detailanalyse eines einzelnen, senkrecht magnetisierten Eisenpartikels beschrieben wurde. Im Gegensatz zu dem separat analysierten Eisenpartikel (vgl. Abbildung 59) wurde diese Kobaltprobe zuvor jedoch in der Ebene in Facettierungsrichtung magnetisiert. 


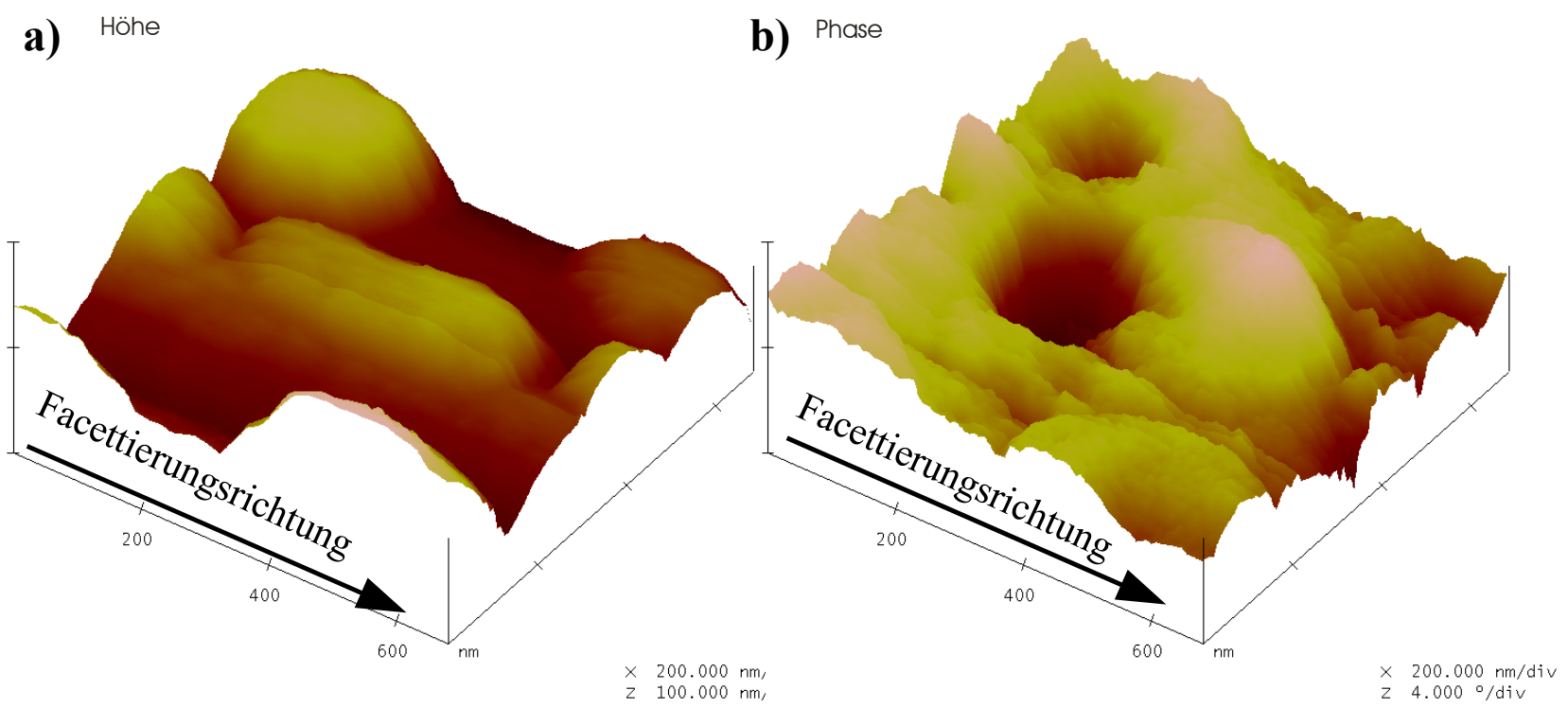

Abbildung 70: a) MFM-Höhen- und b) Phasenabbildung eines runden (oben) und eines elliptischen (Mitte) Kobaltpartikels aus ca. $5 \mathrm{~nm}$ Co-Drähten nach $2 \mathrm{~h}$ bei $550^{\circ} \mathrm{C}$; remanent magnetisiert in Facettierungsrichtung.

Der Zusammenhang von Mikrostruktur und magnetischer Antwort wird beim Magnetisierungsverhalten der getemperten Drähte also zusätzlich deutlich. Die AFM- bzw. MFM-Untersuchungen haben deutlich gemacht, dass die Nanodrähte in eine perlenkettenartige Aufreihung von separierten, weitgehend runden Partikeln eines mittleren Durchmessers von $235 \mathrm{~nm}$ zerfallen sind. Der Vergleich der Magnetisierungskurven im wie hergestellten Zustand (Abbildung 45) mit denen nach der Temperung (Abbildung 71) auf Grundlage der vorgenannten mikrostrukturellen Ergebnisse zeigt wie zuvor beim System Eisen die Kopplung von bestimmten Materialeigenschaften, hier dem

a)

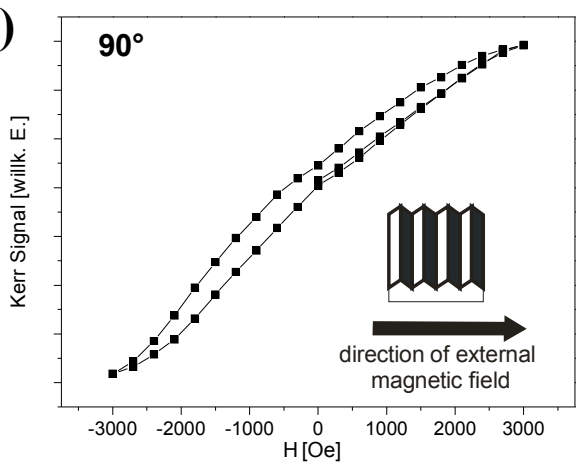

b)

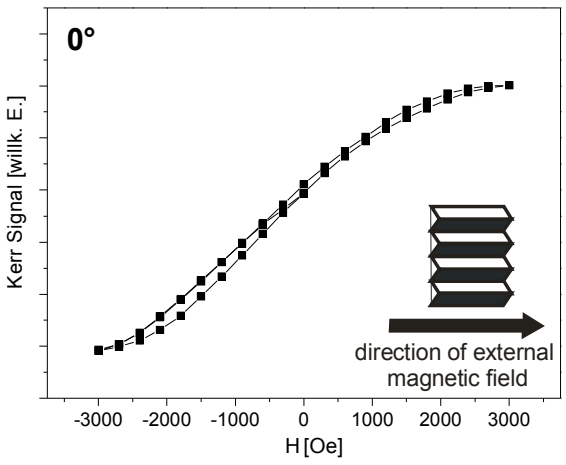

Abbildung 71: MOKE: Magnetisierungsverhalten von ca. 5 nm dicken Co-Drähten auf facettiertem $\mathrm{Al}_{2} \mathrm{O}_{3}$ nach Tempern für $2 \mathrm{~h}$ bei $550^{\circ} \mathrm{C}$. a) senkrecht und b) parallel zur Facettierungsrichtung.

Magnetisierungsverhalten und der Mikrostruktur, welches eine wesentliche Zielsetzung der Materialphysik ist. Die separierten, in der Substratebene runden Partikel zeigen keine Anisotropie in der Wechselwirkung mit dem externen Magnetfeld. Die Magnetisierung folgt sowohl senkrecht als auch parallel zur Facettierungsrichtung weitgehend remanenzfrei dem äußeren Feld. Die uniaxiale Formanisotropie durch die Ausdehnung in Facettierungsrichtung ist beim Zerfall in runde Partikel verschwunden. Die Kristallanisotropie hat bezüglich der leichten und schweren Magnetisierungsrichtung in einzelnen Partikeln Einfluss. Durch die polykristalline, regellose Anordnung der Partikel ist sie aber mesoskopisch und makroskopisch weniger relevant. 
Des Weiteren wurden zum Vergleich Untersuchungen an Drähten mit 20nm Ausgangsschichtdicke durchgeführt. Durch die höhere Bedeckung ist hier ein Zerfall in separierte Partikel behindert (Abbildung 72). Die Materialmenge ist so groß, dass die Facetten nach der Temperung vollständig von Kobalt-Körnern mit stark vergrößertem Aspektverhältnis in Facettierungsrichtung bedeckt werden.
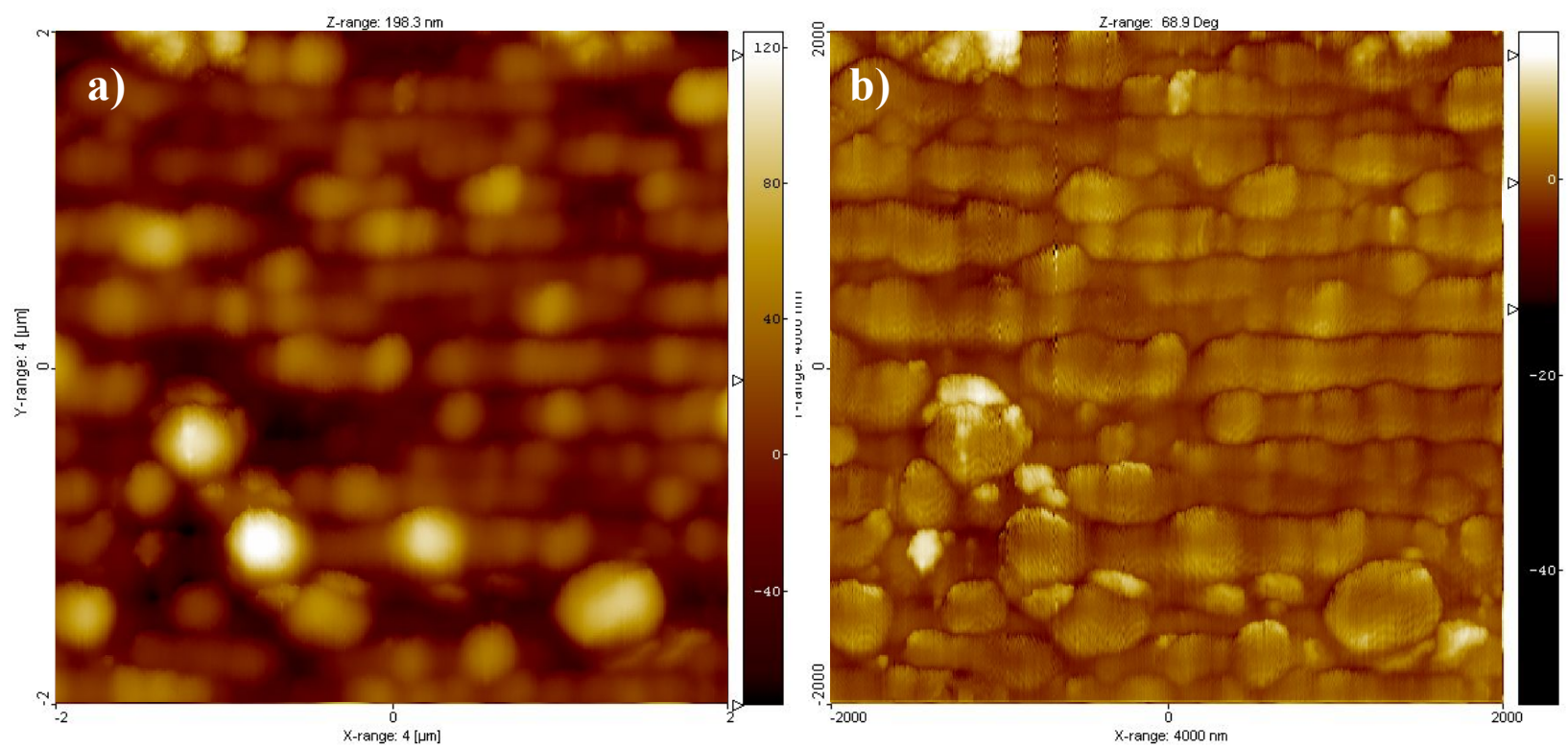

Abbildung 72: a) MFM-Höhen- und b) Phaseninformation von ca. 20nm Co-Drähten, $2 \mathrm{~h}$ bei $550^{\circ} \mathrm{C}$ getempert;

Probe nicht magnetisiert.

Durch die Temperung diffundiert hier das Material auch auf die abgeschattete Facettenseite. Der Materialtransport ist insgesamt sehr hoch, weswegen durch Vergröberung sehr große Höhendifferenzen entstehen. Das Phasenbild (Abbildung 72b) zeigt dies auf der facettierten Unterlage in diesem Fall deutlicher als die Höhendarstellung (Abbildung 72a), da die Höhendifferenzen hier weniger dominant sind.

Eine $\mathrm{zu}$ große Materialmenge führt also zu einer vollständigen Bedeckung der facettierten Unterlage. Die Ausrichtung der entstandenen Körner erfolgt entlang der Facettierungsrichtung. Diese erstrecken sich in dieser Richtung über mehrere Mikrometer. Lateral ist durch die Facettentäler oft eine Begrenzung gegeben, so dass Körner hohen Aspektverhältnisses entstehen. Aus der Höhendarstellung (Abbildung 72a) wird deutlich, dass die Reifung zu großen runden Partikeln mit einer starken Ausdehnung in Richtung der Substratnormalen einhergeht. Insgesamt bleibt aber festzustellen, dass auch bei einer längeren Temperung aus dem beobachteten Gefüge aufgrund der zu großen Ausgangsbedeckung keine Separierung in Partikel der gewünschten Größenordnung zu erreichen ist.

Entsprechend wird die magnetische Formanisotropie im Fall der ca. 20nm dicken Drähte durch die Temperung nicht vollständig aufgehoben. Die mittels AFM beobachteten Veränderungen durch die Temperung spiegeln sich im Magnetisierungsverhalten wieder. Es verbleibt bei Feldrichtung parallel zur Facettierungsrichtung eine deutliche Hysterese, die auf ausgedehnte ferromagnetische Domänen mit leichter Richtung in dieser Orientierung schließen lässt (Abbildung 73b). Ein Vergleich mit dem Magnetisierungsverhalten der Probe vor der Temperung (Abbildung 48) zeigt aber den Übergang von einer ideal quadratischen Hysterese, bei der die gesamte Probe gleichzeitig schaltet, hin zu einem gescherten Verlauf, wobei das Verhältnis $M_{r} / M_{s}$ von 1 auf 0,6 sinkt. Ein wesentlicher Teil der Magnetisierungseinstellung erfolgt durch Rotation von Domänen, was zum 
schrägen Verlauf beiträgt. Ein Vergleich der Kurven für die senkrechte Richtung vor und nach der Temperung ergibt hingegen eine Aufweitung der Hysterese. Dies ist konsistent mit der Beobachtung, dass die Ausdehnung der Körner in senkrechter Richtung zur Facettierungsrichtung zugenommen hat, da eine Ausbreitung des Kobalts über die Facettenkämme hinweg stattgefunden hat.

a)

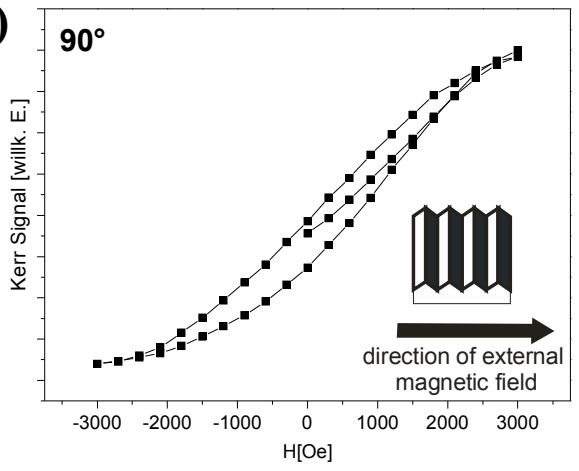

b)

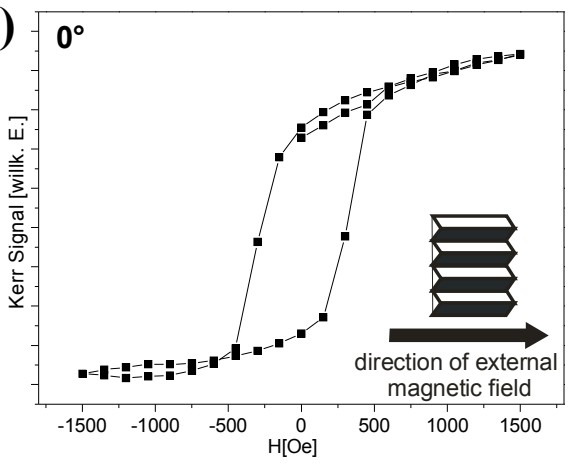

Abbildung 73: Veränderung des Magnetisierungsverhaltens durch Temperung von ca. 20nm dicken Co-Drähten für $2 \mathrm{~h}$ bei $550^{\circ} \mathrm{C}$. a) senkrecht und b) parallel zur Facettierungsrichtung.

Die Veränderung des Magnetisierungsverhaltens in Abhängigkeit von der Temperatur erlaubt Rückschlüsse auf mögliche Oxidation. Die Kopplung des Spins des Kobalts an der Grenzfläche an mögliches Kobaltoxid, bekannt als „exchange-bias“-Effekt, wirkt sich in einer Verschiebungen der Hysterese auf der Abszisse [Mei 56]. Durch temperaturabhängige Magnetisierungsmessungen mittels SQUID bei Feld- und Nullfeldkühlung bis 5K konnten keine derartigen Verschiebung gefunden werden, was den Schluss zulässt, dass die oberflächliche Oxidation des Kobalts bei der ex-situ Charakterisierung keinen wesentlichen Einfluss nimmt und somit auch für die Gestaltbildung beim Zerfall der Drähte in perlenkettenartig aufgereihte separierte Partikel keine grundlegende Relevanz hat.

Zusamenfassend lässt sich feststellen, dass durch Deposition unter einem flachen Winkel erfolgreich dünne $(<20 \mathrm{~nm})$, homogene Nanodrähte der ferromagnetisch ordnenden Materialien Eisen und Kobalt auf die facettierten Substrate deponiert werden und somit die Facetten gezielt zum Übertrag der Periodizität und Ausdehnung auf lineare, magnetische Strukturen genutzt werden konnten. Die ausgezeichnete Form der Drähte, nämlich die extremen Aspektverhältnisse von Länge zu Breite, spiegeln sich in deren Magnetisierungsverhalten wieder, das von uniaxialer Formanisotropie dominiert wird. Das Wachstumsverhalten und damit die Oberflächendiffusion, insbesondere das Diffusionsverhalten an der Barriere der Facettenkämme, ist entscheidend für die Morphologie der Drähte.

Durch Tempern im UHV können diese Nanodrähte in Reihen von separierten, halb-sphärischen Nanopartikeln überführt werden. Leitwertmessungen ergaben eine Unterbrechung des Stromtransports oberhalb von $550^{\circ} \mathrm{C}$ in Übereinstimmung mit den Analysen der Oberflächenstrukturen durch SEM und MFM, die für Kobalt nach Tempern bei $550^{\circ} \mathrm{C}$ und für Eisen bei $600^{\circ} \mathrm{C}$ deutlich den Zerfall der Drähte zeigen. Dabei deuten Untersuchungen bei Temperaturen im Frühstadium der Gestaltänderung an, dass eine sukzessive Aufrauung wesentlich für den Mechanismus der Umformung ist. Der Verlust der anisotropen Form geht einher mit dem Übergang zu einem makroskopisch winkelunabhängigen Magnetisierungsverhalten. MFM-Untersuchungen zeigen die Abhängigkeit der mikroskopischen Magnetisierbarkeit von der Form der einzelnen Partikel und deuten teilweise magnetostatische Kopplung benachbarter Teilchen an. Außerdem konnte ein 
Zusammenhang zwischen Facetten- und damit Drahtbreite und Partikeldurchmesser und Separationsdistanz nachgewiesen werden. Daher kann durch eine gut kontrollierte Nanodrahtbreite und bis zu einer gewissen Grenzschichtdicke (von ca. 15-20nm) auch durch die Drahtdicke die erzielbare Partikelgröße und Separationsdistanz eingestellt werden, was weiterführende Untersuchungen zum magnetischen Kopplungsverhalten ermöglicht. Ein stärker gerichtetes Depositionsverfahren ermöglicht außerdem die Abscheidung von Drahtbreiten entsprechend der geometrischen Abschattung, wodurch auch kleinere Partikelgrößen und -abstände auf gleicher Substratmorphologie möglich sind. 


\section{Transfer der Periodizität in Vielfachschichtsysteme}

Im diesem Kapitel werden Ergebnisse und Analysen zu Schichtpaketen der Materialkombinationen Eisen/Gold, Eisen/Silber und Kobalt/Kohlenstoff dargestellt. Ziel ist, den Einfluss einer periodischen Facettierung des Substrats im Bereich von $\sim 100 \mathrm{~nm}$ auf das Wachstum und die thermische Stabilität sowie die thermisch induzierte Selbstordnung in nicht-mischenden Schichtsystemen [Her 99], [Tro 00] zu untersuchen. Die gewählte Periodizität und die Einzelschichtdicken wurden im Hinblick auf die zu erwartenden Zerfallstemperaturen der Schichtsysteme bei Temperung auf Grundlage der Erfahrungen auf glatten Substraten gewählt. Ein typischer Schichtaufbau bestand aus 20-100 Doppellagen von 10nm Edelmetall und 2nm Eisen, bzw. 10nm Kohlenstoff und 2nm Kobalt.

Von entsprechenden Schichtsystemen der Komponenten Eisen/Silber bzw. Eisen/Gold auf glatten Substraten ist bekannt, dass die Mikrostruktur durch starkes Säulenwachstum geprägt ist. Die inneren Grenzflächen sind trotz großer Fehlpassungen kohärent, was zu einer starken Krümmung der Grenzflächen führt, um die resultierenden Spannungen zu reduzieren. Die kohärente Grenzflächen zwischen Eisen und Edelmetall basieren auf der Nishiyama-Wassermann-Beziehung [Bor 02a]. Durch den strukturellen Übertrag entstehen Säulen einer Breite von $~ 30 \mathrm{~nm}$ parallel zur Substratnormalen, die im System Eisen/Gold der Wachstumssäulenbreite entsprechen. Im System Eisen/Silber liegen Wachstumssäulenbreiten von $80-90 \mathrm{~nm}$ vor, die jedoch aus mehreren zusammenhängenden strukturellen Bereichen bestehen. Nach dem thermisch induzierten Zerfall des Schichtaufbaus ordnen sich Eisenpartikel eines Durchmessers von ca. 10nm meistenteils in den Grenzen der Wachstumssäulen perlenkettenartig aufgereiht, senkrecht zur Schicht an [Tro 00].

Um umfassende Aussagen zum Wachstum der Vielfachschichten auf facettierten Substraten treffen zu können, wurden neben röntgendiffraktometrischen Messungen sowohl Oberflächenanalysen mittels AFM als auch Querschnittsanalysen mittels TEM und STEM durchgeführt.

\subsection{Deposition von Vielfachschichtsystemen auf facettierte Substrate}

\subsubsection{Gefügeänderung durch Substratfacettierung}

Durch die Oberflächenmorphologie des Substrats kann sowohl das Gefüge als auch die Struktur einer aufgebrachten dünnen Schicht stark beeinflusst werden. Bei entsprechendem Wachstumsverhalten und geeigneten Wachstumsbedingungen kann dies die Struktur betreffend bis hin zu einer Ausrichtung der Kristallstruktur an der Gestalt des Substrat führen (Graphoepitaxie).

Die Facetten können aber auch auf die Anordnung von Körnern und Korngrenzen Einfluss nehmen, ohne dass des zu einer Ausrichtung der Struktur kommt. Die Oberflächenanalyse ermöglicht die Beurteilung des Einflusses des nanostrukturierten Substrats auf das Gefüges der Schichtsysteme.

Eine Abbildung von Körnern und Gefüge mit charakteristischen Längen unter 50nm war bisher mit Rasterelektronenmikroskopie SEM nur schwer zugänglich. Andere Rasterverfahren, wie die 
Rastertunnel- (STM) oder Rasterkraftmikroskopie (AFM) konnten insbesondere bei glatten Schichten nur sehr begrenzt helfen, da der Spitzendurchmesser hier die laterale Auflösung stark begrenzt und zu Abbildungsfehlern führen kann [Str 04]. Durch ein modernes LEO SUPRA 35 stand jedoch ein SEM zur Verfügung, mit dessen Detailauflösung im Nanometerbereich eine Oberflächenabbildung und -analyse auch von sehr glatten metallischen Schichtpaketen auf glatten und facettierten Saphirsubstraten möglich wurde und somit der Nachweis von Veränderungen des Gefüges durch die Substratfacettierung erbracht werden konnte.
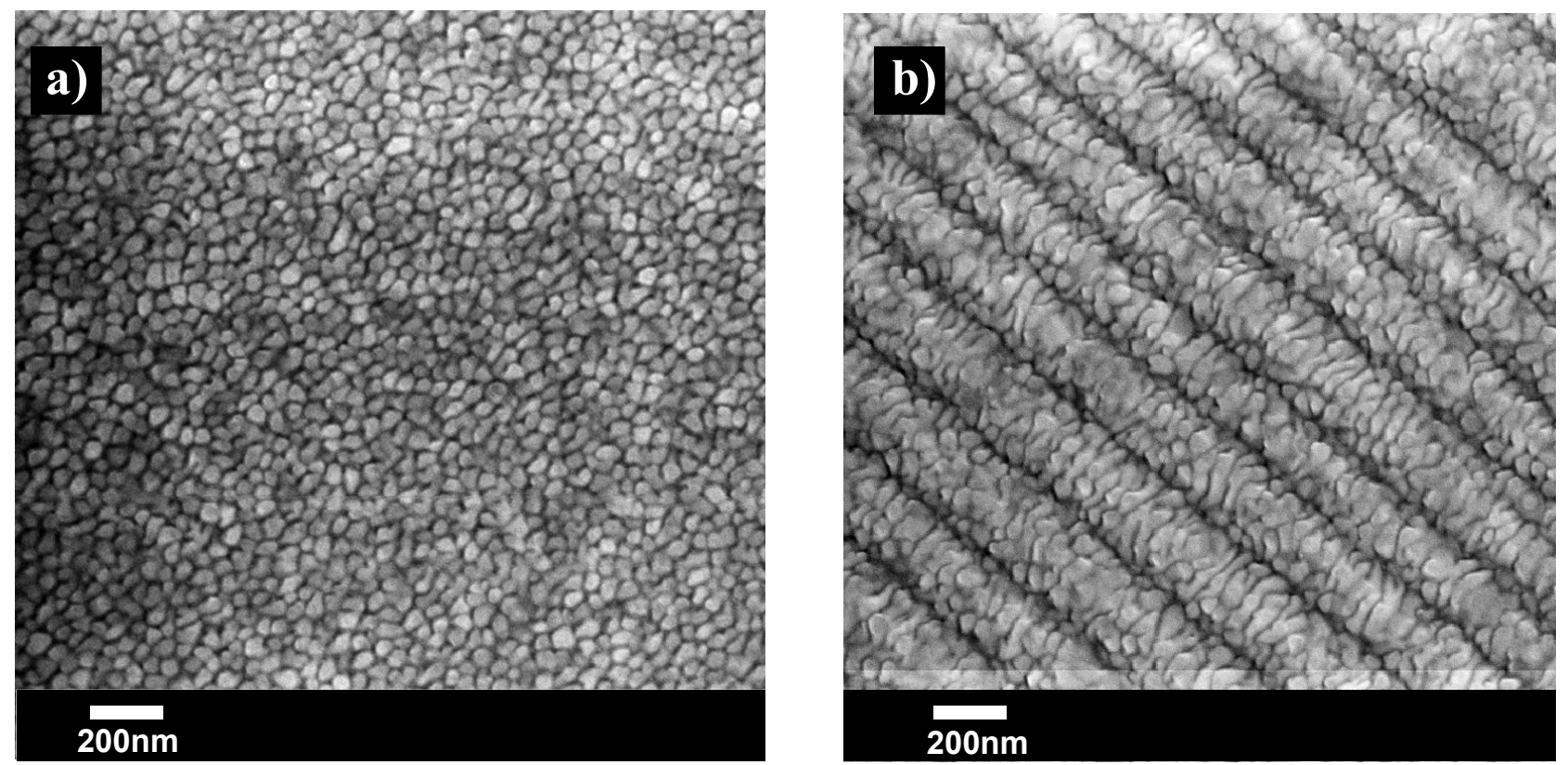

Abbildung 74: SEM-Abbildung: Eisen/Gold-Schichtpaket 50x(2nm Fe/10nm Au) a) auf glattem und b) auf facettiertem $\mathrm{Al}_{2} \mathrm{O}_{3}$.

Abbildung 74 zeigt vergleichend zwei SEM-Abbildungen des Gefüges an der Oberfläche eines Schichtpakets mit 50 Doppellagen der Kombination $10 \mathrm{~nm}$ Gold/2nm Eisen, auf glattem und auf facettiertem $\mathrm{Al}_{2} \mathrm{O}_{3}$. Die Bereiche des Schichtpakets auf glattem Substrat haben eine polygone Form, meist annähernd fünfeckig oder sechseckig. Es ist keine bevorzugte Ausrichtung erkennbar. Der mittlere Durchmesser beträgt 54nm. Damit ist der Durchmesser an der Oberfläche größer als die mittels TEM-Querschnittspräparationen bestimmte Breite der Wachstumssäulen im System Eisen/Gold von ca. 30nm [Her 99], [Tro 00]. Da sich die Wachstumssäulen mit zunehmender Schichtdicke jedoch durch das Überwachsen effektive verbreitern und in einer Querschnittsaufnahme nicht notwendigerweise der maximale Durchmesser bestimmt wird, ist davon auszugehen, dass die Bereiche in Abbildung 74a die Wachstumssäulen repräsentieren.

Wie stark der Einfluss eines quasiperiodisch strukturierten Substrat ist, zeigt ein Vergleich mit der Abbildung 74b. Die Veränderungen lassen sich folgendermaßen zusammenfassen:

- Von den unterliegenden Facetten sind durch eine Gefügetrennung besonders die Facettentäler gut erkennbar. Dort finden sich auch bevorzugt sehr kleine Bereiche mit einem Durchmesser von $15 \mathrm{~nm}$ bis zu $30 \mathrm{~nm}$.

- Die Facettenkämme und die Facettenseiten sind von runden Bereichen bedeckt, die den gleichen Durchmesser haben, wie Wachstumssäulen auf glatten Substraten. Die facettierte Unterlage hat aber einen ordnenden Einfluss auf die Lage der Wachstumssäulen. Während auf dem glatten Substrat eine statistische Verteilung der Säulen beobachtet wurde, überdecken auf der facettierten Unterlage typischerweise 4-5 Körner eine einzelne Facette in der Weise, dass ihre Mittel punkte auf einer Linie senkrecht zur Facettierungsrichtung liegen (siehe auch Abbildung 75). 
- An einigen Stellen, insbesondere in Bereichen, an denen die Facetten besonderes schmal sind, treten stark anisotrope Formen der Oberflächenmorphologie auf, da nahezu die gesamte Facette von einem zusammenhängenden Bereich überwachsen wird. Daher finden sich Aspektverhältnisse von Länge (in Facettierungsrichtung) zu Breite von bis zu 6. Diese Bereiche zeigen bei genauer Betrachtung aber Einbuchtungen, die die Vermutung nahelegen, dass es sich um Zusammenschlüsse von runden Wachstumssäulen handelt (Abbildung 75).

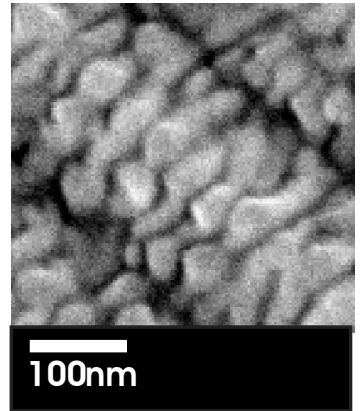

Abbildung 75: SEMAnalyse: Überspannen eines einzelnen Facettendachs.

Zum Vergleich zeigt Abbildung 76 die Oberfläche eines Eisen/Silber-Schichtsystems auf einem facettierten Substrat. Die Oberflächenmorphologie des Eisen/Silber-Schichtpakets ist geprägt von einer Vielzahl runder, kleiner Körner $(\sim 20-40 \mathrm{~nm})$, die in Form und Orientierung unabhängig von der unterliegenden Substratfacettierung sind. Auf glatten Substraten unterscheiden sich die Systeme Eisen/Gold und Eisen/Silber in zwei Punkten. In Eisen/Silber sind die Wachstumssäulen mit 80$100 \mathrm{~nm}$ deutlich breiter und in den Wachstumssäulen werden unterschiedlich orientierte Körner beobachtet. Der geringe Kontrast in SEM-Abbildungen von Eisen/Silber-Schichtsystemen, wie beispielsweise in Abbildung 76, aber auch die geringe Rauigkeit von $<1,5 \mathrm{~nm}$ (bestimmt mittels AFM) zeigt, dass die sichtbaren Bereiche deutlich flacher als an der Oberseite der Eisen/GoldSysteme sind. Ein direkter Rückschluss auf die Wachstumssäulen scheint hier nicht gegeben, vielmehr könnten ein Volmer-Weber-Wachstum der obersten Silberschicht zur beobachteten Oberflächenmorphologie führen. Dieser Wachstumsmodus wurde u.a. auch von Vrijmoeth et al. für Silber auf Silber(111) bei Raumtemperatur beobachtet [Vri 94]. Die Facettentäler sind auch im System Eisen/Silber sehr deutliche Trennlinien, was die maximale Ausdehnung der Wachstumssäulen auf den Facetten begrenzt.

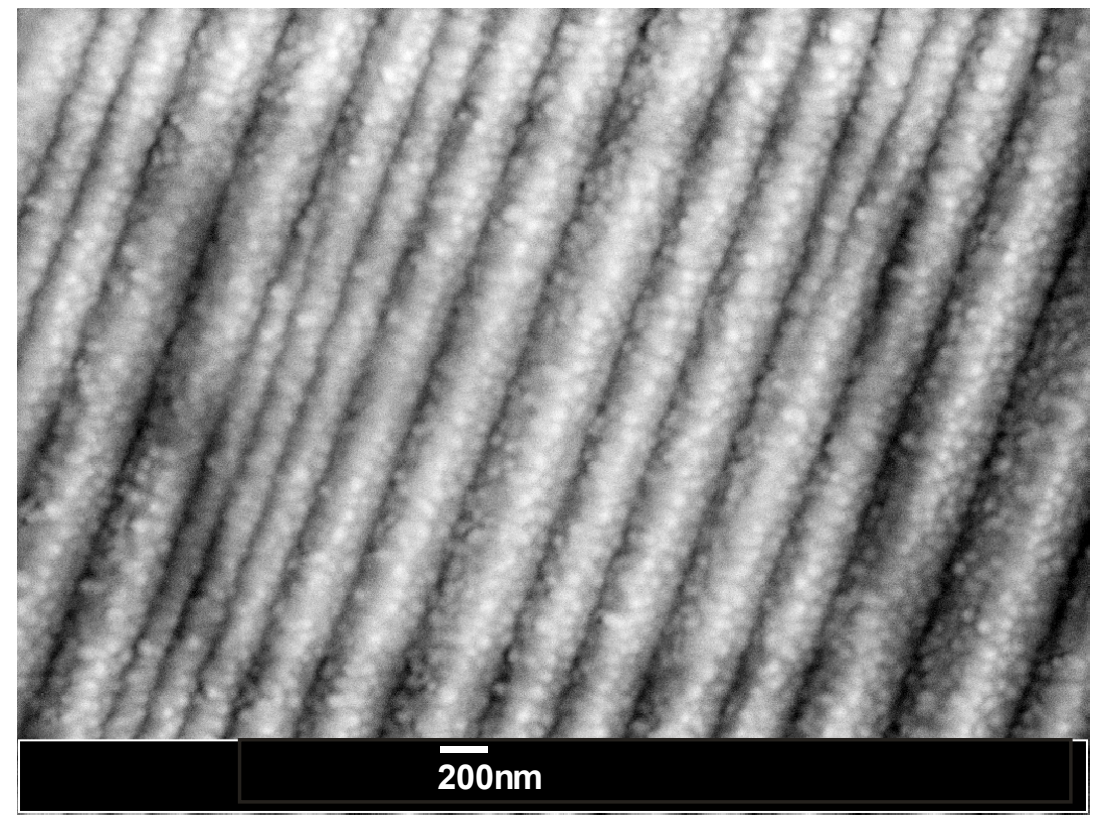

Abbildung 76: SEM-Analyse: 50x(2nm Eisen/10nm Silber)-Schichtsystem auffacettiertem $\mathrm{Al}_{2} \mathrm{O}_{3}$. 
Eine Abhängigkeit der Oberflächenmorphologie von der Orientierung der Facettierungsrichtung beim Beschichtungsprozess (vertikal oder horizontal vgl. Abbildung 2) konnte nicht beobachtet werden.

Um zu überprüfen, ob die mesoskopische Welligkeit an der Oberfläche der Schichtsysteme noch der der Facettierung des Substrat entspricht, wurden AFM-Analysen durchgeführt. Abbildung 77 und Abbildung 78 zeigen exemplarisch dreidimensionale Darstellungen der AFM-Daten für Schichtpakete mit jeweils 20 Doppellagen Eisen/Silber bzw. Eisen/Gold. Diese bestätigen zunächst die Aussagen, die aus den hochaufgelösten SEM-Bildern gewonnen werden konnten. Die Oberfläche der Eisen/Gold-Schichtpakete ist rauer. Die Facettenkämme und -täler sind bei Materialkombinationen auch auf der Oberfläche des 240nm dicken Schichtsystems sehr ausgeprägt.

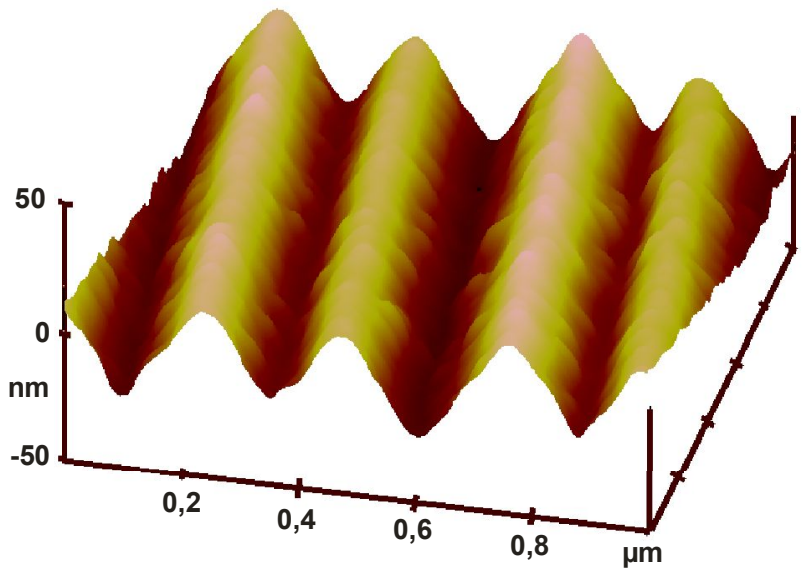

Abbildung 77: AFM-Höhendaten: 20x(2nm Fe/10nm Ag) auf facettiertem $\mathrm{Al}_{2} \mathrm{O}_{3}$.

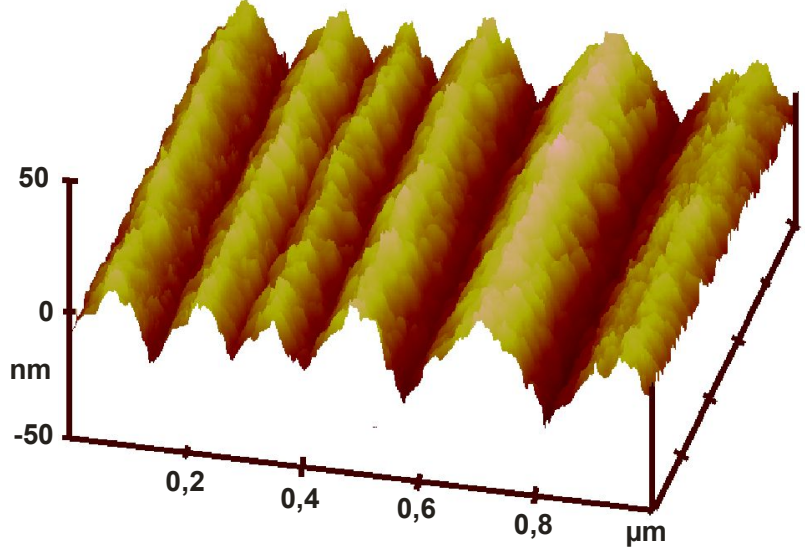

Abbildung 78: AFM-Höhendaten: 20x(2nm Fe/10nm Au) auf facettiertem $\mathrm{Al}_{2} \mathrm{O}_{3}$.

An der Oberseite des Eisen/Silber-Schichtsystems wirkt die Oberfläche in den SEM-Bildern feinkörniger und glatter, die Facettenmorphologie ist insgesamt abgerundet, aber in den Tälern deutlich getrennt. Die feinkörnige Bedeckung ist lateral durch den abbildungsbegrenzenden Spitzenradius der AFM-Spitze kaum aufzulösen ist. Eine Analyse der Höhe der Facettierung an der Oberseite der Schichtpakete ergibt mit Werten bis zu 49nm für das System Eisen/Gold und 48nm für Eisen/Silber, dass die künstliche Rauigkeit des Substrat komplett bis zur Oberfläche erhalten bleibt. Abbildung 79 zeigt einen typischen Schnitt senkrecht zur Facettierungsrichtung für die AFM-Höhendaten des Eisen/Silber-Schichtsytems auf facettiertem Saphir dargestellt in Abbildung 77. 


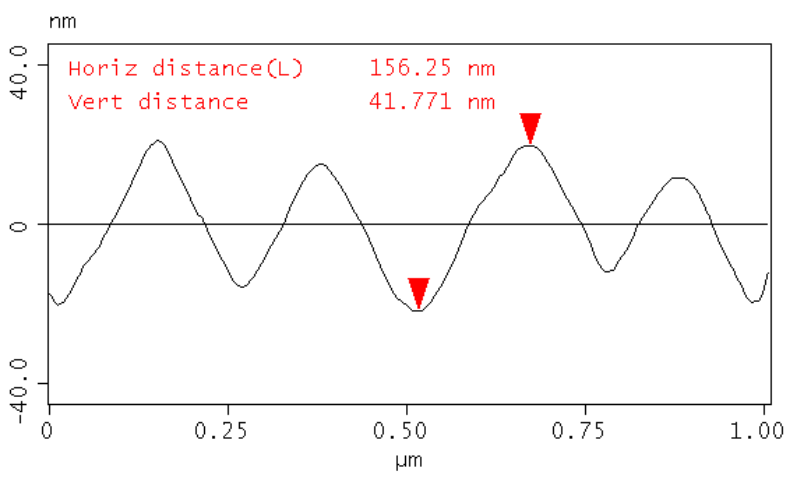

Abbildung 79: AFM-Schnittdarstellung zu Höhendaten aus Abbildung 77, 20x(2nm Eisen/10nm Silber) auf facettiertem $\mathrm{Al}_{2} \mathrm{O}_{3}$.
Die SEM- und AFM-Analysen zeigen also insbesondere für das Eisen/Gold-Schichtsystem Veränderungen der Form und Anordnung der Körner bzw. Wachstumssäulen an der Oberfläche durch die nanostrukturierte Unterlage des facettierten m-plane $\mathrm{Al}_{2} \mathrm{O}_{3}$-Substrat. Die Abbildungen der Oberseite der Schichtpakete geben insbesondere für das Eisen/Silber-System Hinweise auf einen dreidimensionalen Wachstumsmodus. 


\subsubsection{Wachstum der Vielfachschichtsysteme}

Um die Ursachen dieser Gefügeveränderung besser zu verstehen, ist es von Interesse, das Wachstumsverhalten während des Schichtwachstums zu untersuchen. Die in-situ Charakterisierung des Schichtwachstums mit Beugungsmethoden, wie z.B. RHEED, erfordert eine sehr glatte Unterlage, weshalb dieses Verfahren für die Untersuchung des Wachstums auf facettierten Substraten nicht geeignet ist. Dennoch konnten durch einen neu konzipierten Messaufbau mit entsprechender Auswertungselektronik und individuell angepasster Software Informationen zum Schichtwachstum während der Deposition gewonnen werden. Durch die Ausnutzung der elektrischen Leitfähigkeit der deponierten Materialien konnten Perkolationsschwellen bestimmt werden. Die in-situ Leitfähigkeitsbestimmung ermöglicht darüber hinaus indirekt Aussagen über das Aufwachsverhalten von Eisen auf den Edelmetallen.

\section{Edelmetall-Eisen Schichtsysteme}

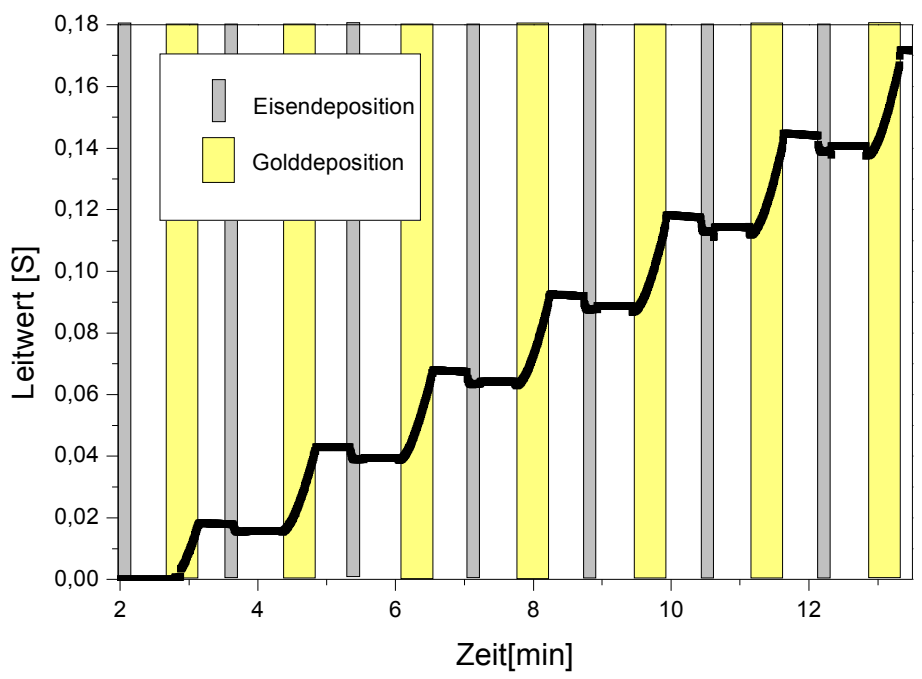

Abbildung 80: Beginn des Leitwertverlaufs während der Deposition von $50 x(2 \mathrm{nmFe} / 10 \mathrm{~nm} \mathrm{Au})$ auffacettiertes $\mathrm{Al}_{2} \mathrm{O}_{3}$.
Abbildung 80 zeigt die Veränderung des Leitwerts des deponierten Schichtsystems zu Beginn der Herstellung von 50 Doppellagen Eisen und Gold. Der Depositionsverlauf stellt sich als ein Abfolge von 4 wiederkehrenden Phasen dar, die zur besseren Unterscheidung in Abbildung 80 farblich unterlegt sind:

1. $11 \mathrm{~s}$; Deposition von $2 \mathrm{~nm}$ Eisen

2. 30s; Depositionspause

3. $29 \mathrm{~s}$; Deposition von $10 \mathrm{~nm}$ Gold

4. 30s; Depositionspause

Während der Depositionspausen, sind beide Quellen durch eine Blende abgedeckt, so dass der Einfluss des Plasmas der Magnetronquellen auf die Leitwertmessung weitestgehend minimiert wird.

Abbildung 81 zeigt den genauen Leitwertverlauf während einer Gold- bzw. Eisenschichtdeposition. In der Golddepositionsphase wächst der Leitwert stetig an, zunächst mit zunehmender Steigung gefolgt von einem weitgehend linearen Anstieg.

Während der Depositionspausen ist der Leitwert konstant, es treten aber beim Übergang zu den Depositionsphasen kleinere Sprünge auf. Hieran zeigt sich der Einfluss des Plasmas der Quellen bzw. nicht neutraler Atome während des Beschichtungsprozesses. Dieser ist während des gesamten Beschichtungsprozesses konstant, wirkt jedoch in der Leitwertdarstellung nach langen Zeiten bzw. bei großen Schichtdicken besonders deutlich, da hier der Widerstand der Schicht geringer ist. Im Folgenden wurde der zu einer bestimmten Schichtdicke korrespondierende Leitwert immer in der sich anschließenden Depositionspause ermittelt. 
Während der Deposition von Eisen fällt der Leitwert in den ersten Sekunden der Depositon deutlich ab. Nach ca. 1/3 der Depositionszeit entsprechend einer Dicke von etwa $0,7 \mathrm{~nm}$ geht der Leitwertverlauf in einen sehr flachen Anstieg über. Dieser ist bei weitem nicht ausreichend, um die vorhergehende Reduktion zu kompensieren, so dass es bei der Deposition jeder Eisenlage insgesamt zu einer Verschlechterung der Leitfähigkeit des Schichtpakets kommt.

Die Entwicklung des Leitwerts ist im System Eisen/Silber praktische analog, wie die Abbildung 82 zeigt.

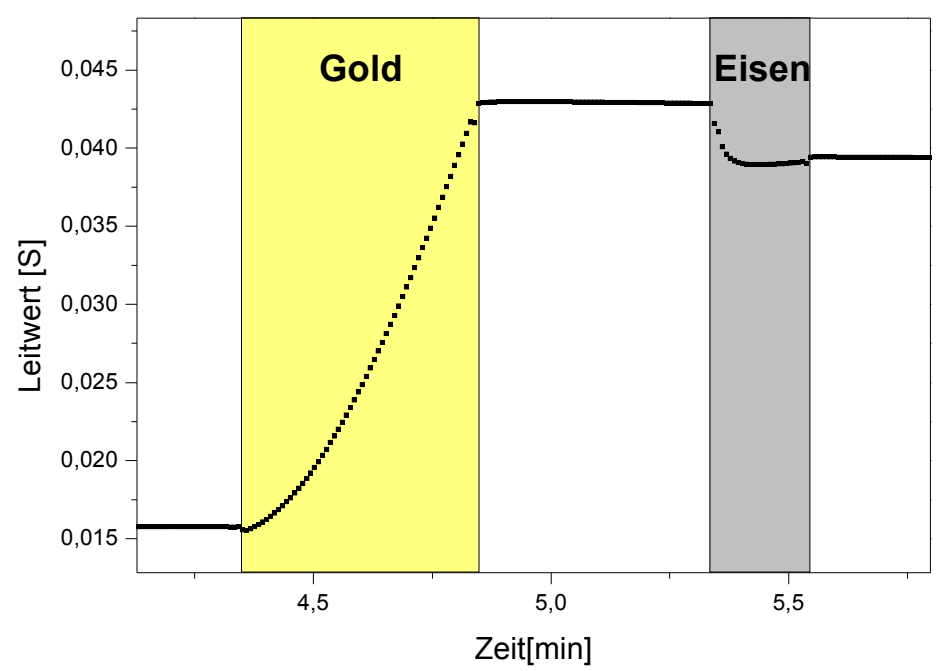

Abbildung 81: Leitwertverlauf während einzelner Gold- bzw. Eisendeposition.

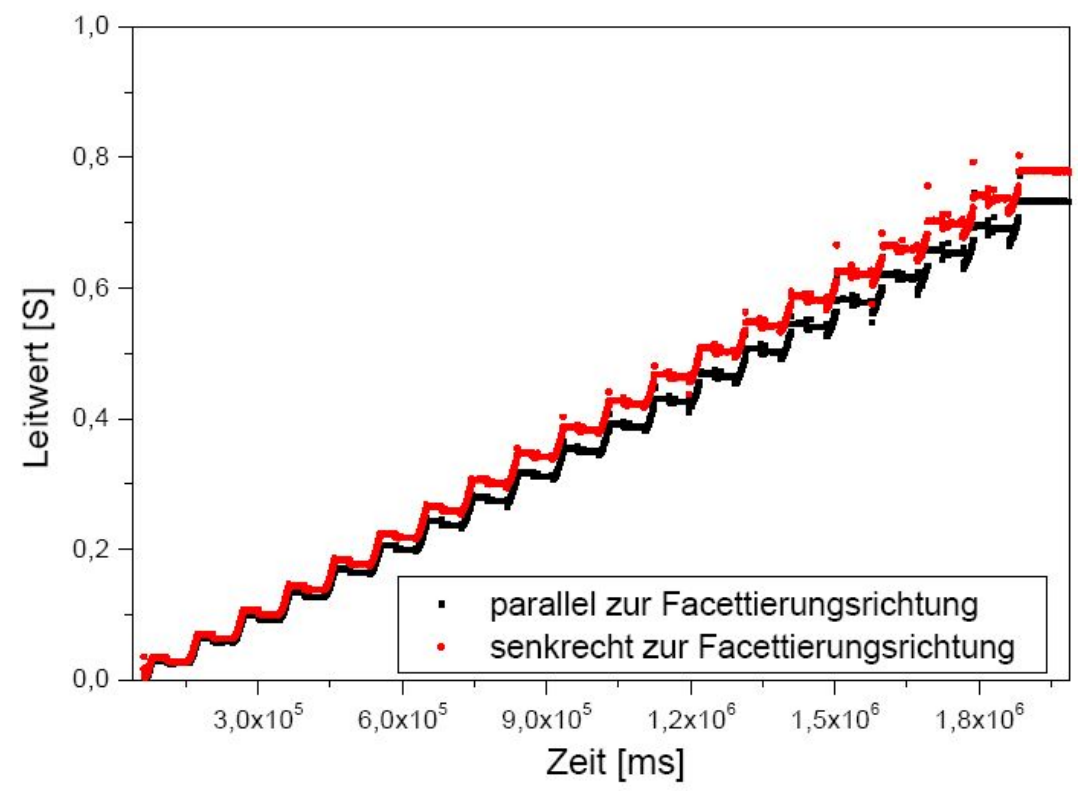

Abbildung 82: Leitwertentwicklung während der Deposition von $20 x(2 \mathrm{~nm} \mathrm{Fe} / 10 \mathrm{~nm} \mathrm{Ag})$ auf facettiertes $\mathrm{Al}_{2} \mathrm{O}_{3} ; 30$ s Pause nach jeder Einzellage.

Die Leitwertmessungen während der Deposition zeigen einen fast linearen Anstieg des Leitwerts mit der Lagenzahl und damit mit der Schichtdicke.

Eine besondere Aussagekraft hat der Zeitpunkt, zu dem erstmals eine Leitfähigkeit messbar ist. Abbildung 83 zeigt den Beginn der Deposition für beide Schichtsysteme, jeweils in beiden Messrichtungen. Während der Deposition der ersten Eisenschicht ist in beiden Systemen keine Leitfähigkeit messbar (dieser Bereich ist in Abbildung 83a und b nicht dargestellt). Erst nach der Deposition von weiteren 3,1nm Gold bzw. 2,2nm Silber findet Perkolation statt. Der Beginn der Deposition mit Silber führte schon nach wenigen Sekunden entsprechend einer Schichtdicke $<1,5 \mathrm{~nm}$ zu messbaren Leitfähigkeit. 

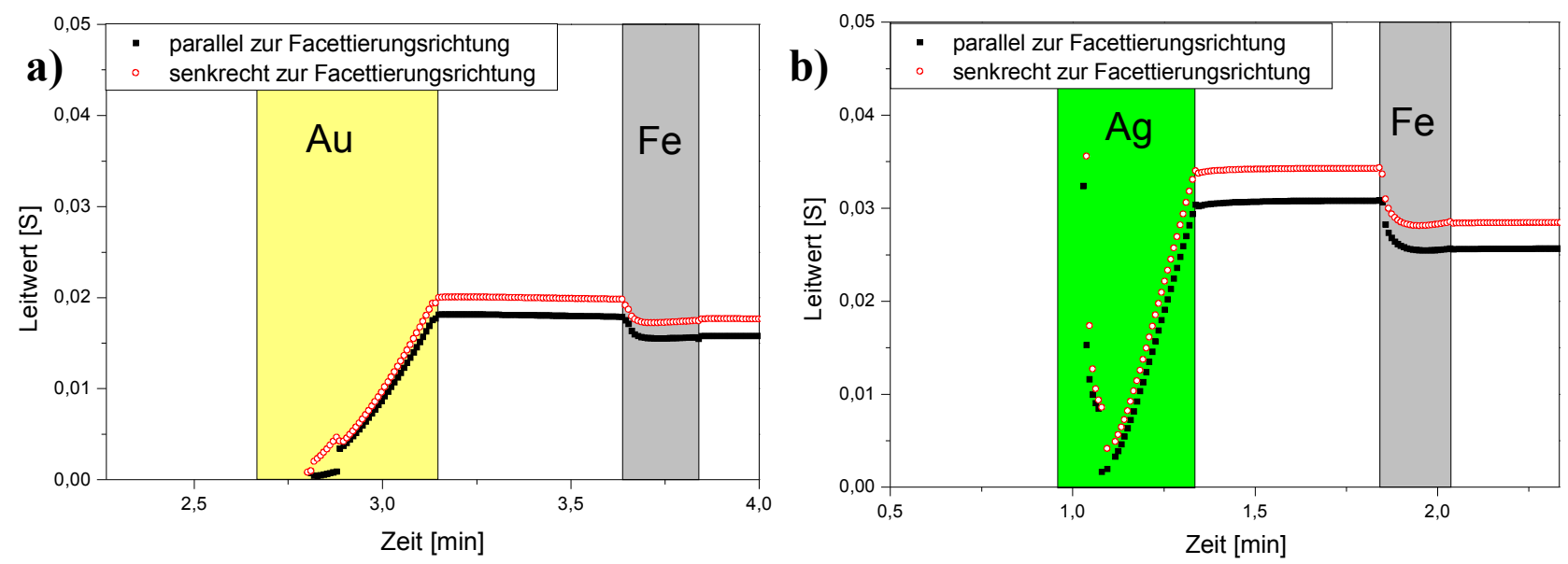

Abbildung 83: Perkolationsschwelle bei der Deposition von a) (2nm Eisen/10 $\mathrm{nm}$ Gold)-Schichtpaketen bzw. b) (2nm Eisen/10nm Silber)-Schichtpaketen auffacettiertem Saphir nach $2 \mathrm{~nm}$ Eisen plus 3,1nm Gold bzw. $2 \mathrm{~nm}$ Eisen plus 2,2nm Silber. Die Depositionsphase der ersten Eisenschicht liegt außerhalb des Darstellungsbereichs.

Die Tatsache, dass in beiden Systemen keine Leitfähigkeit während der Deposition der ersten 2nm Eisen gemessen wird, zeigt, dass das Eisen im Sinne eines Volmer-Weber-Wachstums unter Inselbildung auf dem facettierten Saphirsubstrat aufwächst. Die Eiseninseln sind dabei nicht durch einen durchgängigen Pfad miteinander verbunden.

Lairson et al. finden für Eisen auf $\mathrm{MgO}(001)$ bei Raumtemperatur Volmer-Weber-Wachstum und beobachteten bis zu einer Schichtdicke von $3 \mathrm{~nm}$ kein Zusammenwachsen der Eiseninseln [Lai 95].

Schon bei geringer Kontaktfäche würde ein Tunnelstrom beobachtet werden können [Neu 62], [Bol 04]. Erst durch die Deposition einer zum Eisen vergleichbaren Menge Edelmetall entstehen leitfähige Pfade. Dabei

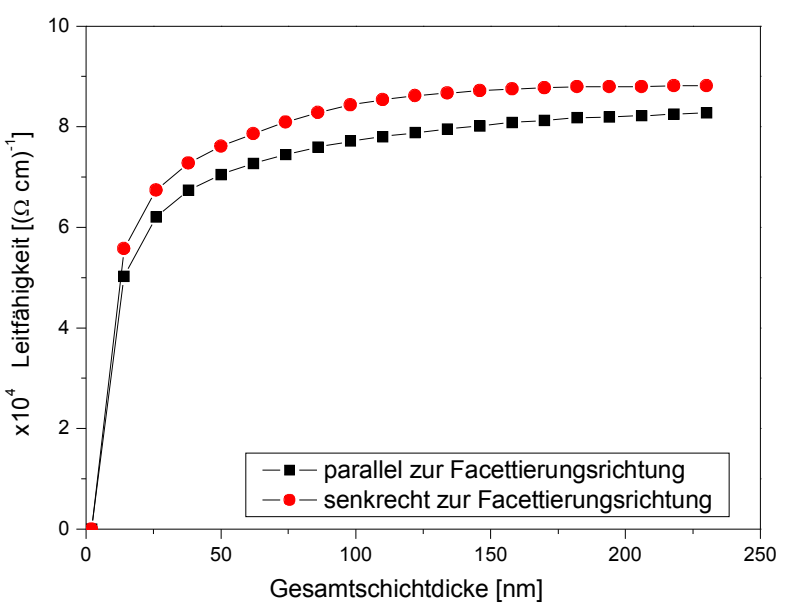

Abbildung 84: Leitfähigkeitsentwicklung beider Messrichtungen während der Deposition von $20 x(2 \mathrm{~nm} \mathrm{Fe} / 10 \mathrm{~nm} \mathrm{Ag})$ auf facettiertem $\mathrm{Al}_{2} \mathrm{O}_{3}$. erhält man bei der Deposition von Silber wegen der größeren spezifischen Leitfähigkeit einen höheren Leitwert als bei Gold. Der erste leitfähige Pfad wird für beide Messrichtungen bei gleicher Schichtdicke beobachtet. Dies gilt sowohl für Eisen/Silber als auch für Eisen/Gold. Zwischen den beiden Messrichtungen zeigen sich bei beiden Systemen eine weitgehend konstante Leitfähigkeitsdifferenz. Der Leitwert senkrecht zur Facettierungsrichtung liegt im weiteren Verlauf - wahrscheinlich durch die effektive Verbreiterung des Leitungsquerschnitts durch die Projektion der Depositionsmaske in Materialstromrichtung immer über der in Richtung der Facetten. Eine Abschätzung dieses Effekts alleine aus der geometrischen Verbreiterung ergibt 15-17,5\% höhere Leitwerte für die Messrichtung senkrecht zu den Facetten. Beobachtet wird aber nur eine Leitwertdifferenz von ca. 8\%. Zwar kommt es auch in der Messrichtung parallel zu den Facetten zu einer sehr geringe Verbreiterung, dennoch kann die verbleibende Differenz möglicherweise einer stärkeren Behinderung des Transport senkrecht zur Facettierungsrichtung zugeschrieben werden.

Unter Berücksichtigung der genauen geometrischen Abmessungen des Beschichtungsbereichs, des Beschichtungsquerschnitts, der aktuellen Schichtdicke und der Leiterlänge ist es möglich, aus den 
ermittelten Leitwerten den spezifischen Widerstand bzw. die Leitfähigkeit der Schicht zu ermitteln und deren Veränderung während des Schichtwachstums zu analysieren. Abbildung 84 zeigt den weitgehend parallelen Verlauf der Leitfähigkeit in beiden Messrichtungen exemplarisch für das System Eisen/Silber auf einem facettierten Substrat. Insgesamt ist festzustellen, dass die anisotrope Oberflächenmorphologie des Substrats auf das Transportverhalten keinen wesentlichen Einfluss hat.
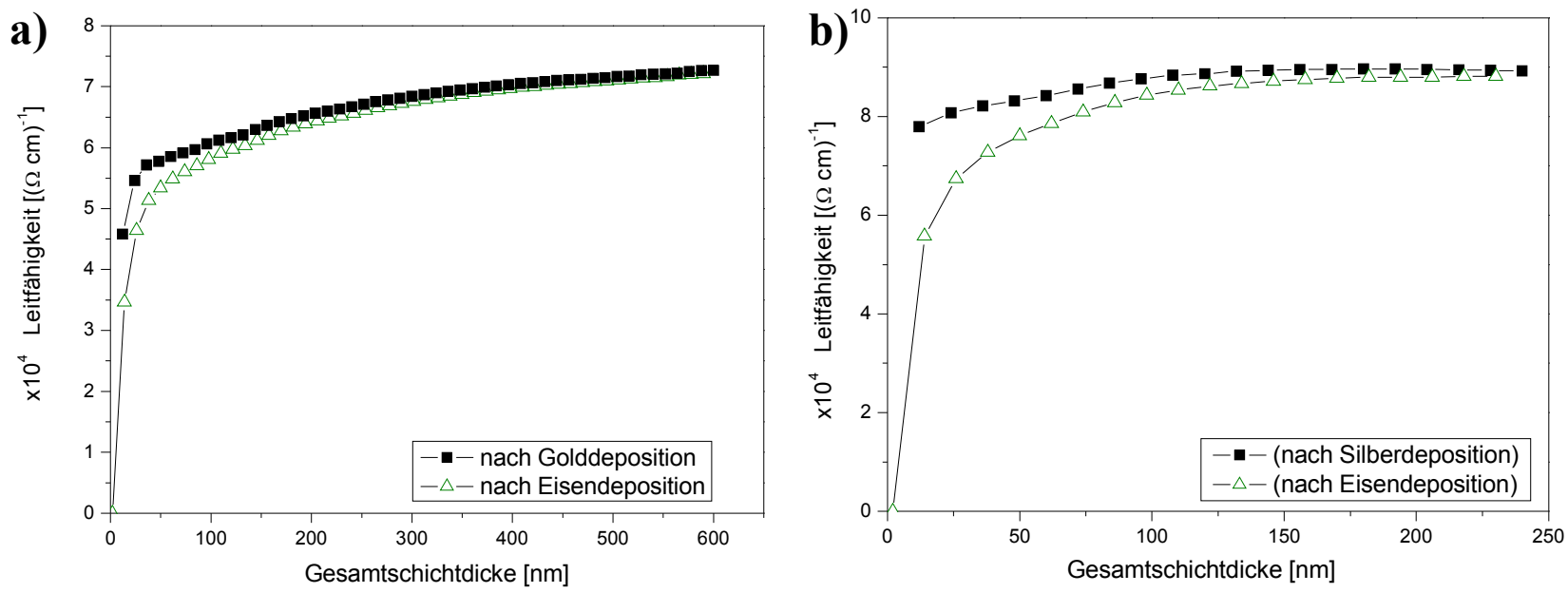

Abbildung 85: Leitfähigkeitsentwicklung während der Deposition von a) 50x(2nm Fe/10nm Au) und während der Deposition von b) $20 x(2 \mathrm{~nm} \mathrm{Fe} / 10 \mathrm{~nm} \mathrm{Ag)} \mathrm{auffacettiertem} \mathrm{Substrat,} \mathrm{senkrecht} \mathrm{zur} \mathrm{Facettierungsrichtung.}$

Abbildung 85 zeigt die während der Depositionspause ermittelten Leitfähigkeiten, getrennt in die Messwerte nach einer Gold- bzw. Silberschichtdeposion und nach einer Eisenschichtdepositon. Die Daten der Eisendeposition liegen während der gesamten Depositionsdauer unter denen der Goldbzw. Silberdeposition. Das verdeutlicht, dass das Aufbringen von Eisen während des gesamten Beschichtungsprozesses zur Verschlechterung der Leitfähigkeit führt.

Die Leitfähigkeit zeigt eine Sättigung. Während im Eisen/Gold-System auch nach 50 Doppellagen eine geringe Steigerung der Leitfähigkeit über den Wert von $7,2 \cdot 10^{4}(\Omega \mathrm{cm})^{-1}$ nachweisbar ist, wird im Fall der Kombination mit dem Edelmetall Silber schon nach 13 Doppelschichten eine maximale Leitfähigkeit von $8,4 \cdot 10^{4}(\Omega \mathrm{cm})^{-1}$ erreicht. Dass auch nach 50 Doppellagen im System Eisen/Gold eine Steigerung der Leitfähigkeit beobachtet wird, erstaunt insofern, als dass durch die hohe Anzahl an Grenzflächen ein normaler Größeneffekt im Sinne eines Fuchs-Sondheimer [Fuc 38], [Son 52] Ansatzes unterdrückt sein sollte. Durch weitere Lagen sollte es nicht zu einer weiteren Zunahme einer effektiven mittleren freien Weglänge kommen können, da schon an den inneren Grenzflächen viele Streuereignisse auftreten werden. Die weitere Steigerung der Leitfähigkeit deutet auf eine hohe Kohärenz der Grenzflächen zwischen Eisen und Gold hin, so dass nur wenige Streuereignisse an diesen Grenzflächen stattfinden.

Die gemessenen Leitfähigkeiten liegen etwa eine Größenordnung unter den spezifischen Leitfähigkeiten der Konstituenten der Schichtsysteme, wie sie von verschiedenen Autoren berichtet werden $\sigma_{\mathrm{Ag}}=68,0 \cdot 10^{4}(\Omega \mathrm{cm})^{-1}, \quad \sigma_{\mathrm{Au}}=49,0 \cdot 10^{4}(\Omega \mathrm{cm})^{-1}, \quad \sigma_{\mathrm{Fe}}=11,6 \cdot 10^{4}(\Omega \mathrm{cm})^{-1} \quad[$ Lan 82]. Durch Spannungen, die kolumnare Mikrostruktur und zahlreiche Defekte, wie sie typisch für gesputterte dünne Schichten sind, ist eine deutliche Reduktion der Leitfähigkeit unter die Werte von Kompaktmaterial zu erwarten [Lan 57]. Die große Zahl an Wachstumssäulengrenzen in den vorliegenden Systemen in Kombination mit den künstlichen Grenzflächen durch die Multilagenschichtung trägt sicher massgeblich zur Reduktion der Leitfähigkeit bei. In der Gesamtheit wird dadurch der Stromtransport senkrecht zur als auch in der Ebene durch Streuereignisse an diesen ausgezeichneten Grenzen effektiv behindert [May 70], [Van 87]. 
Abbildung 86 zeigt die Leitfähigkeitsentwicklung während der ersten 3 Doppellagen der Deposition eines Eisen/Silber-Schichtsystems auf ein glattes $(10 \overline{1} 0)-\mathrm{Al}_{2} \mathrm{O}_{3}$ Substrat. Während der Abscheidung der ersten Eisenschicht ist wieder keine Leitfähigkeit messbar.

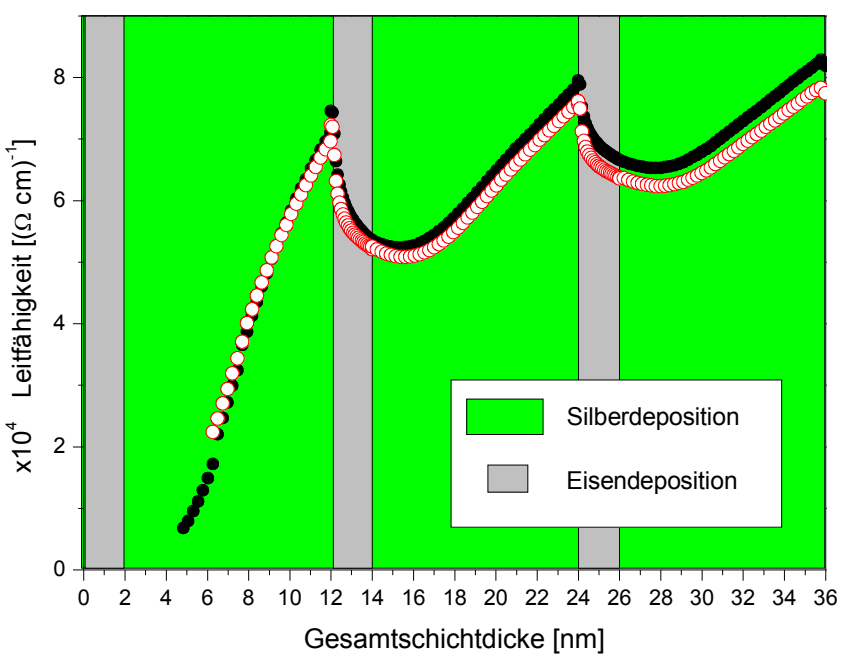

Abbildung 86: Leitfähigkeit während der ersten drei Doppellagen der Deposition eines 20x(2nm Fe/10nm Ag) Schichtsystems auf glattem m-plane $\mathrm{Al}_{2} \mathrm{O}_{3}$; beide Messrichtungen.

Im Anschluss an die Deposition des Eisens ist bei der Deposition von Silber ein Minimum zu beobachten, dem erst nach $3 \mathrm{~nm} \mathrm{Ag} \mathrm{eine} \mathrm{lineare} \mathrm{Leitfähigkeitszunahme}$ folgt. Hierbei ist zunächst $\mathrm{zu}$ berücksichtigen, dass bei der Bestimmung der Leitfähigkeit eine lineare Zunahme der Dicke mit der Zeit vorausgesetzt wurde. Sowohl eine Überschätzung der deponierten Dicke als auch eine Schicht, die nicht zur Leitfähigkeit beiträgt, können zu einer Absenkung der so bestimmten Leitfähigkeit führen. Beide Effekte sind bei diesen Messungen zu erwarten. Zum Anfang der Deposition wird die Targetspannung angelegt, d.h. die Depositionsrate erreicht erst nach einer gewissen Zeit ihren stabilen Wert. Weiterhin erfolgt die Deposition von Silber auf einer zunächst durch das VolmerWeber- resp. Stranski-Krastanov-Wachstum rauen Eisenoberfläche [Vol 26], [Str 38].

Der Einfluss der Rauigkeit der Oberfläche wird im Weiteren im Vergleich zu anderen Faktoren diskutiert. Wahrscheinlich ist die Wechselwirkung der Elektronenkonfiguration des 3d-Übergangmetalls Eisen mit der neu aufwachsenden Edelmetallschicht der dominierende Effekt. Um die Wirkung des veränderten Potentials der Eisenatome effektiv abzuschirmen (Frideloszillationen), ist eine gewisse Schichtdicke an Edelmetall notwendig. Erst wenn diese Schichtdicke auf die Eisenschicht deponiert wurde, erfolgt eine erneute Leitfähigkeitsteigerung.

Bei der Deposition von Eisen auf Silber bzw. Gold wird sogar ein starkes Absinken des Leitwertes beobachtet. Die folgende Diskussion soll helfen, diese unterschiedlichen Einflussfaktoren darzustellen und ihren Stellenwert bei der beobachteten Leitwertreduktion einzuschätzen.

Die Deposition von Atomen eines Elements auf die Oberfläche eines anderen führt zu einer verstärkten Oberfächenstreuung. Der dominierende Streumechanismus ist dabei durch die Art der Adatome gegeben. Wenn diese eine andere Valenzelektronenzahl haben, so ist die CoulombStreuung der maßgebliche Effekt [Gre 66], [Gre 66a]. Wenn beide Elemente die gleiche Anzahl an Elektronen pro Atom haben, so ist das Oberflächenpotential nur aufgrund der mikroskopischen Oberflächenaufrauung durch die Adatome verändert.

1) Coulombstörung durch Eisen als starken Streuer:

Es ist bekannt, dass Oberflächenadsorbate anderer Valenz einen sehr starken Einfluss auf das elektrische Leitungsverhalten haben. Im Volumen wurden für Verunreinigungen von Silber bzw. Gold mit Eisen Werte von $8 \mu \Omega \mathrm{cm} /$ at.\% gefunden [Lan 82]. First-Principle Rechnungen von Podloucky et al. [Pod 80] zeigen, dass Eisen die Bandstruktur von Silber sehr stark verändert und daher den elektrischen Transport effektiv stört $(10,8 \mu \Omega \mathrm{cm} /$ at.\%).

Trioni et al. zeigten mittels LDA und LSDA, dass Eisen als Adsorbat an der Oberfläche sogar einen stärkeren Einfluss auf die Leitfähigkeit hat, als wenn es interstitiell eingebaut. Zwar 
wurden die Rechnungen für Aluminium als Trägermaterial durchgeführt, es wird aber ausdrücklich auch Bezug auf die Richtigkeit für Edelmetalle wie Silber und Kupfer genommen. [Tri 01], [Tri 02].

2) Die Morphologie der aufwachsenden Eisenschicht:

Wesentlichen Einfluss auf die Leitfähigkeit einer metallischen dünnen Schicht hat deren Oberflächenbeschaffenheit [Sof 67], [Hof 81], [Tes 86]. Während eine atomar glatte Schicht zu einer maximal spiegelnden Reflexion der Leitungselektronen führt, muss bei Inselbildung inelastische Streuung an der Oberfläche berücksichtigt werden [Jac 90]. Im Fall einer sehr dünnen Schicht im Bereich der Perkolation muss auch Transport durch induziertes Tunneln berücksichtigt werden.

Es ist bekannt, dass das Wachstum von Eisen auf $\mathrm{Au}(111)$ zunächst durch Inselbildung erfolgt. [Bul 04] Hierbei nukleieren die Eiseninseln an den Knicken der im Fischgrätenmuster rekonstruierten Oberfläche [Str 92]. Dabei werden zunächst Gold-Atome an diesen Stellen ausgetauscht, woraufhin die Nukleation der Inseln eben dort erfolgt [Mey 96], [Shi 04].

3) Absputtern von Silber bzw. Gold durch auftreffende Eisen-Atome (vgl. z.B. [Stu 01], [Kah 00]):

Es erscheint unwahrscheinlich, dass Absputtern von Ag bzw. Au bei den hergestellten Schichten ein wesentlicher Prozess ist. Im Gegensatz zur Laserdeposition ist die Energieverteilung beim Magnetron-Sputtern deutlich schärfer, zudem liegt die durchschnittliche kinetische Energie der auf die Schicht auftreffenden Atome im Bereich von 0,2-5eV. Die Sputterausbeute für Silber ist zwar im Vergleich zu anderen Elementen sehr groß, jedoch reicht die kinetische Energie der auftreffenden Atome in der Regel nicht aus, um die Bindungsenergie zu überwinden. Zudem beobachtet man keinen signifikanten Unterschied zwischen den Raten reiner Silberschichten und Schichtpaketen. Würde ein wesentlicher Teil der Silberatome vom deponierten Eisen bei der Multilagenherstellung abgetragen, so wäre eine reduzierte Silbereinzelschichtdicke die Folge. Dies konnte aber nicht beobachtet werden.

4) Eindiffusion von Eisen in die unterliegende Edelmetallage, Segregation in Korngrenzen

Eine weitere Möglichkeit wäre eine schnelle Diffusion der Eisenatome in Korn- bzw. Wachstumssäulengrenzen oder „triple junctions" in der darunterliegenden Edelmetallschicht. Die Oberflächenanalyse der Schichtpakete mittels SEM (z.B. Abbildung 74) in Kombination mit den Kenntnissen zum kolumnaren Schichtaufbau vergleichbarer Schichtsysteme auf glatten Substraten aus TEM-Untersuchungen [Her 99], [Tro 00] lassen dies trotz der thermodynamischen Barrieren einer großen Mischungsentalphie (für Eisen/Silber $\Delta \mathrm{H}_{\text {mix }}=+42 \mathrm{KJ} / \mathrm{mol}$ [Ami 04]) möglich erscheinen. Die Zahl von Wachstumssäulengrenzen ist bedingt durch den mittleren Durchmesser von $54 \mathrm{~nm}$ hoch und die polygone Form

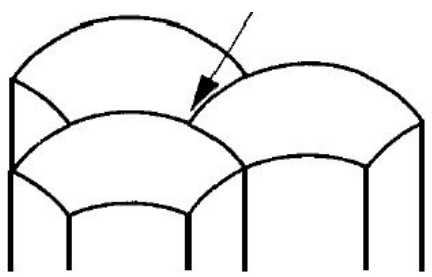

Abbildung 87 Korngrenzen und Tripelpunkte bei gekrümmten Grenzflächen. führt ebenfalls zu einer hohen Dichte an „triple junctions“ (vgl. Abbildung 87).

Um zu klären, ob die Segregation von Eisen an diesen mikrostrukturell ausgezeichneten Punkten in der unterliegende Edelmetalllage einen dominierenden Effekt darstellt und somit für den wesentlichen Teil der Leitfähigkeitsreduktion verantwortlich ist, konnten Untersuchungen an Schichtabfolgen der Kombination Eisen/Silber mittels der Tomographischen Atomsonde durchgeführt werden. Diese bietet die Möglichkeit, Durchmischungen an Grenzflächen auf 
atomarer Skala zu untersuchen [Klu 03]. Außerdem konnte Lang zeigen, dass das Verfahren aufgrund der atomarer Auflösung bei räumlicher Darstellbarkeit gut geeignet ist, um Segregation in Korngrenzen und „triple junctions“" nachzuweisen [Lan 02].

Ein weiterer Prozess, der $\mathrm{zu}$ einer Leitwertminderung führen würde, wäre eine starke Durchmischung der Grenzflächen. Um diese unterschiedlichen Möglichkeiten der Grenzflächendurchmischung und Segregation, zu klären, wurden unterschiedliche Schichtsysteme präpariert.

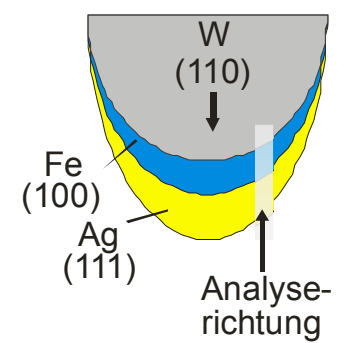

Abbildung 88: Skizze zur Position des Analysevolumens auf der Spitze.
Zunächst wurde mittels Ionenstrahlsputterns ein Doppellage aus Eisen und Silber mit den Einzelschichtdicken 90nm Eisen und 100nm Silber auf eine Wolframspitze aufgebracht (Abbildung 88). Das Probenvolumen wurde dabei bewusst nicht zentral auf der Spitze platziert, um die Wahrscheinlichkeit zur erhöhen, Korngrenzen im Analysevolumen vorzufinden. Lang konnte im System $\mathrm{Cu} / \mathrm{Au}$ zeigen, dass eine solche Verschiebung aus dem Zentrum der Spitze eine deutliche Erhöhung der Korngrenzdichte zur Folge hat [Lan 02]. Das Gefüge kann sich aber von dem planarer Schichtsysteme unterscheiden (vgl. Kapitel 2.8). a)

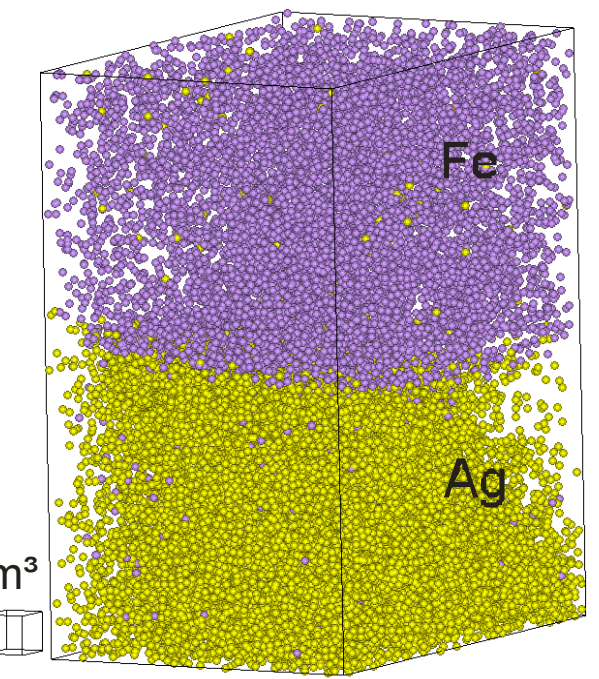

Analysiertes Probenvolumen b)

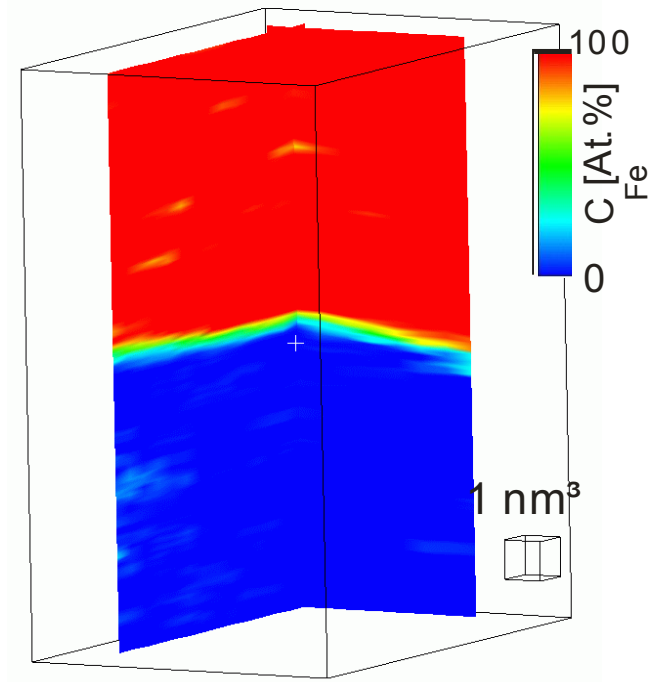

2D-Konzentrationsschnitte

Abbildung 89: a) Atompositionen (Eisen violett und Silber gelb) und b) Konzentrationsverteilung (Schnitte) im rekonstruierten Probenvolumen aus TAP-Analyse eines Bilayers 90nm Eisen/100nm Silber.

Abbildung 89 zeigt die Elementverteilung im analysierten Probenvolumen und im rechten Teil zwei senkrecht aufeinander stehende zweidimensionale Konzentrationsschnitte. In der Analyse sind unterschiedlich orientierte Bereiche erkennbar. Eine Ausrichtung, in der über größere Bereiche regelmäßig Netzebenabstände erkennbar sind, konnte nicht erzielt werden. Dennoch ist von der Kristallinität beider Metalle auszugehen und die unterscheidbaren Bereiche deuten auf Korngrenzen hin. Ausgehend von der Grenzfläche zwischen Eisen und Silber finden sich im Probenvolumen keine Bereiche, in denen die Konzentration des Fremdelements in der Matrix signifikant erhöht wäre, d.h. eine Segregation oder gar eine Benetzung von Silberkorngrenzen durch Eisen oder aber von Eisenkorngrenzen durch Silber ist somit sehr unwahrscheinlich. 
a)

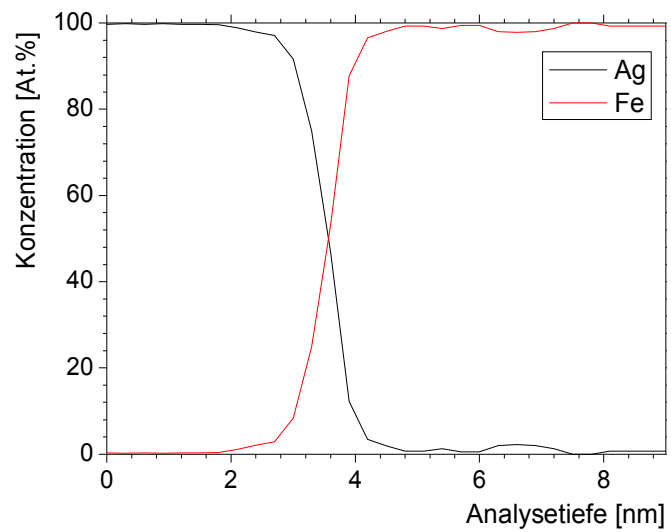

b)

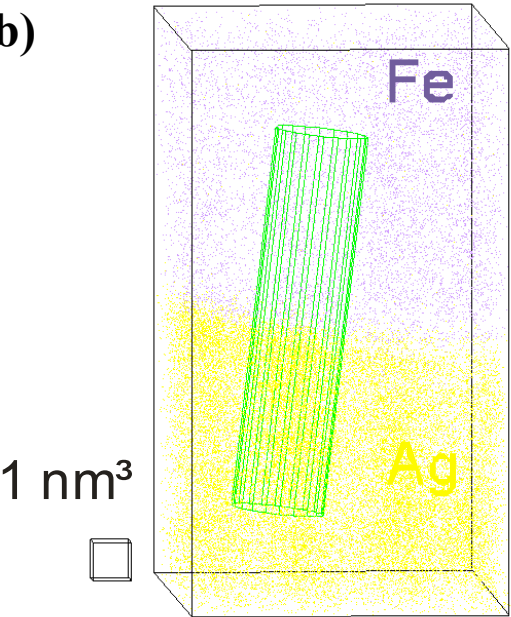

Abbildung 90: TAP-Analyse: a) Konzentrationsverlauf senkrecht zur Eisen/Silber-Grenzfläche; b) Position, Orientierung und Größe des Analysezylinders für den Konzentrationsverlauf über die Grenzfläche.

Die Konzentrationsschnitte machen aber noch weitere Aussagen möglich. Die Breite der Region, in der der Konzentrationswechsel von Eisen zu Silber erfolgt, ist sehr schmal, d.h. eine starke Durchmischung der Grenzfläche kann für den Fall der Deposition von Silber auf Eisen ausgeschlossen werden. Der komplette Kontrastwechsel von rot (100\% Eisen) zu blau (100\% Silber) erfolgt in einer Region, die schmaler ist als die Höhe des dargestellten Vergleichsvolumens von $1 \mathrm{~nm}$.

In Abbildung 90a ist eine eindimensionale Analyse entlang eines zusätzlichen Anlaysezylinders im Probenvolumen (b) dargestellt, der bei der Auswertung so in das Analysevolumen eingebracht wurde, dass seine Längsachse parallel zur lokalen Grenzflächennormalen verläuft. Der resultierende Verlauf der Konzentrationen für Silber und Eisen ist in der Auftragung abhängig von der Analysetiefe gegeben. Die Profile verlaufen sehr symmetrisch um die Lage der Grenzfläche bei 3,6nm. Die scharfe Grenzfläche und der gleichförmige Profilverlauf für Eisen und Silber bestätigen eine minimale Grenzflächendurchmischung und liefern keine Anzeichen für die Deposition von Material unter die Grenzfläche der anderen Komponente. Die starke Tendenz zur Entmischung im System Eisen/Silber mit einer Mischungsenthalpie von $42 \mathrm{~kJ} / \mathrm{mol}$ in der festen und sogar $28 \mathrm{~kJ} / \mathrm{mol}$ [Ami 04] in der flüssigen Phase bestätigt sich in einem sehr scharfen Grenzflächenverlauf trotz der vergleichsweise hohen Energien der auftreffenden Atome beim Ionenstrahlsputtern.

Insgesamt konnten die Ergebnisse der TAP-Untersuchung also zwei wesentliche Fragestellungen klären. Zum einen konnte gezeigt werden, dass das System Eisen/Silber, selbst bei der Deposition mittels Ionenstrahlsputtern sehr scharfe Grenzflächen bildet. Des weiteren kann ausgeschlossen werden, dass eine signifikante Segregation von Eisen in Silberkorngrenzen auftritt.

Zusammenfassend müssen die vier vorgenannten Erklärungsansätze für die starke Leitfähigkeitsabsenkung bei Eisendeposition wie folgt beurteilt werden. Der Beitrag der CoulombStreuung an der Absenkung ist als dominierend zu bewerten. Dabei ist jedoch die Deposition auf die unterliegende Edelmetallfläche ausreichend und eine signifikante Diffusion von Eisen in die Säulengrenzen und ,triple junction“ wird nicht beobachtet.

Der Einfluss der Oberflächen- und Grenzflächenrauigkeit auf die Leitfähigkeitsentwicklung in einem Schichtpaket ist komplexer. Vergleichende in-situ Leitwertmessungen an Schichtsystemen auf facettierten und glatten Substraten konnten zeigen, dass die künstliche Rauigkeit durch die Facettierung, keinen signifikanten Einfluss auf die Leitfähigkeit des aufgebrachten Schichtsystems 
hat. Aus TEM-Querschnittsuntersuchungen und TAP-Analysen ist bekannt, dass die Grenzflächen Edelmetall-Eisen bzw. Eisen-Edelmetall keine deutlichen Rauigkeitsunterschiede aufweisen. Trotzdem ist die Leitwertveränderung in beiden Fällen sehr unterschiedlich. Jede neu deponierte Edelmetalllage erhöht den Leitwert, jede neu deponierte Eisenlage verringert ihn. Dies lässt den Schluss zu, dass nicht die strukturelle Rauigkeit der Oberfläche, wie sie von Jacob et al. [Jac 90] und Hoffmann et al. [Hof 81] diskutiert wird, wesentlich für die beobachteten Leitwertänderung ist, vielmehr führt die veränderte Valenzelektronenzahl des Eisens gegenüber dem stromtragenden Edelmetall zu einer starken Coulomb-Streuung. Hierin ist folglich der dominierende Effekt in der leitfähigkeitsreduzierenden Wirkung des Eisens zu sehen.

\section{$\underline{\text { Kohlenstoff-Kobalt Schichtsysteme }}$}

Im Gegensatz zu den Edelmetallen Silber und Gold, die sich als gute elektrische Leiter auszeichnen und somit das wesentliche Material für den Stromtransport in den Eisen/Silber- und Eisen/GoldSchichtsystemen darstellen, sind die Leitfähigkeitsverhältnisse in den $2 \mathrm{~nm}$ Kobalt/10nm Kohlenstoff Schichtsystemen umgekehrt. Hier ist die Leitfähigkeit der dünneren Komponente Kobalt mit $16,02 \cdot 10^{4}(\Omega \mathrm{cm})^{-1} \quad$ um einen Faktor 800 höher als die der dickeren amorphen Kohlenstoffschichten $\left(0,02 \cdot 10^{4}(\Omega \mathrm{cm})^{-1}\right.$. Selbst gegenüber der Leitfähigkeit in den Graphit $a-b-E b e n e n$

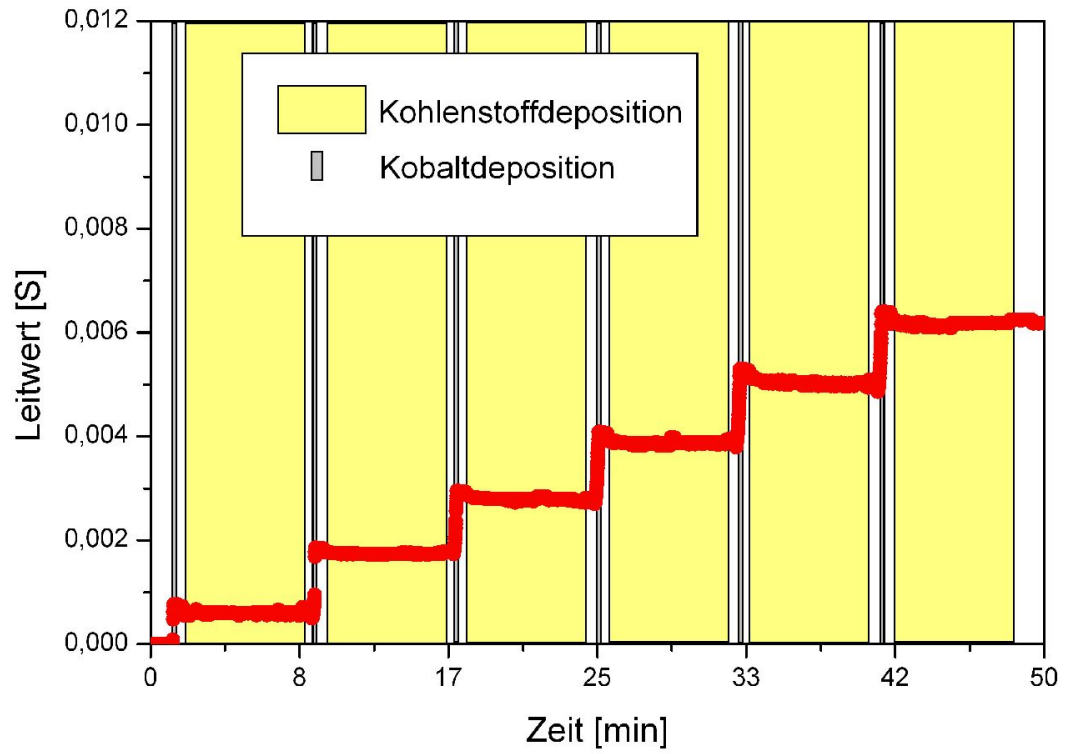

Abbildung 91: In-situ Leitwertmessung während der Deposition eines $6 x(2 \mathrm{~nm} \mathrm{Co} / 10 \mathrm{~nm} \mathrm{C})$ Schichtsystems auf glattem $\mathrm{Al}_{2} \mathrm{O}_{3}$ (Depositionspausen sind weiß unterlegt). $\left(3 \cdot 10^{4}(\Omega \mathrm{cm})^{-1}\right.$ ist die des

Kobalts um etwa den Faktor $\sim 5$ größer. Dies veranschaulicht nur eine obere Grenze für die Leitfähigkeit der Kohlenstoffschichten, es ist jedoch von einer ungeordneten sp ${ }^{2}$-hybridisierten Kohlenstoffschicht auszugehen, deren mittlere Leitfähigkeit deutlich niedriger ist.

Entsprechend erwartet man eine Leitwertsteigerung im Bereich der Kobaltdeposition und nur geringe Veränderungen während der Kohlenstoffdeposition.

Beginnend mit der Deposition von Co ist in Abbildung 91 exemplarisch der Leitwertverlauf während der Deposition eines Vielfachschichtsystems aus $6 \mathrm{x}(2 \mathrm{~nm} \mathrm{Co} / 10 \mathrm{~nm} \mathrm{C})$ auf glattem $\mathrm{Al}_{2} \mathrm{O}_{3}$ dargestellt. Der Leitwertverlauf auf facettiertem $\mathrm{Al}_{2} \mathrm{O}_{3}$ ist vergleichbar. Schon während der ersten $2 \mathrm{~nm}$ Co wird ein Leitwert beobachtet. Steigerungen des Leitwerts sind während jeder Kobaltdeposition erkennbar, wohingegen es durch die Kohlenstoffdeposition zu einer leichten Reduktion in der Leitfähigkeit kommt. Die Depositionszeiten für die Kobaltschichten betrugen nur 13s, wohingegen die Depositionszeiten für den Kohlenstoff, bedingt durch die kleine Sputterausbeute, 400 s umfassten. 
Eine Analyse der Leitwerte während der dreißigsekündigen Depositionspausen nach den jeweiligen Einzelschichten ergibt die in Abbildung 92 dargestellte Leitfähigkeit für eine Deposition auf facettiertes $\mathrm{Al}_{2} \mathrm{O}_{3}$.

Durch die Kohlenstoffdeposition sinkt die Leitfähigkeit des Systems immer ab. Nach 4 Doppellagen ist ein Maximum der Leitfähigkeit von $0,25 \cdot 10^{4}(\Omega \mathrm{cm})^{-1}$ erreicht, im Folgenden sinkt die Leitfähigkeit mit höheren Schichtdicken. Hierfür ist die sehr schlechte Leitfähigkeit durch die Kohlenstofflagen ursächlich. Der Transport durch Lagen, die weiter vom Substrat und damit von den Kontakten der Leitfähigkeitsmessung entfernt sind, ist durch die 10nm dicken Kohlenstoff-Zwischenschichten

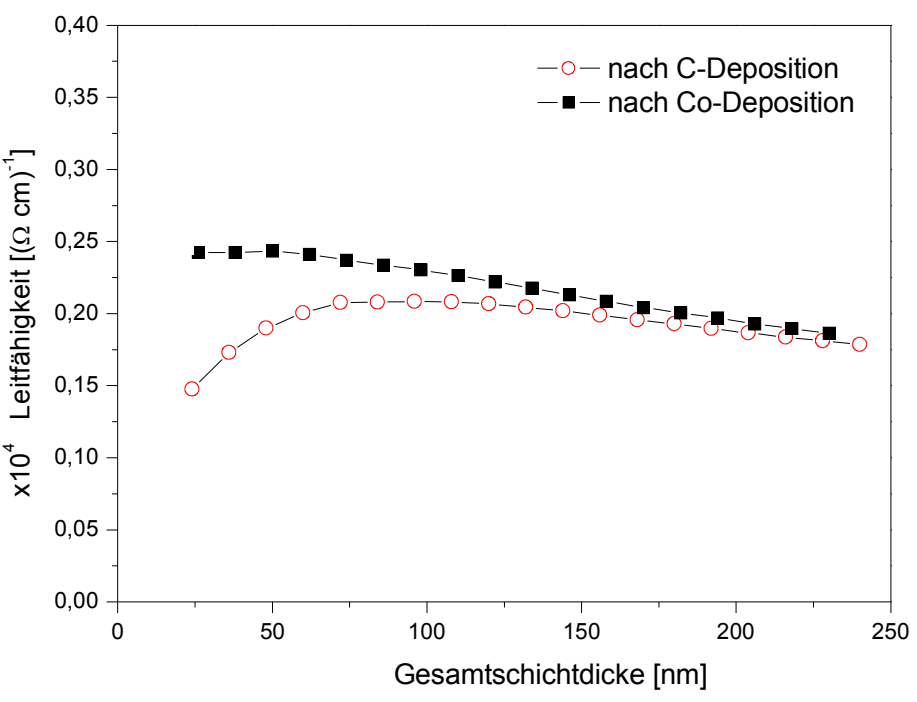

Abbildung 92: Leitfähigkeitsveränderung während der Deposition eines Schichtsystems aus 20x(2nm Co/10nm C) auf facettiertem $\mathrm{Al}_{2} \mathrm{O}_{3}$. unterdrückt, nur ein geringer Anteil kann des Stroms durch wenige leitfähige Kanäle (Kurzschlüsse) in die darüber liegenden Kobaltschichten gelangen. Die Kohlenstoff-Zwischenschichten verhindert also die gute Stromverteilung im Schichtsystem. 


\subsubsection{Innerer Aufbau und Periodizität der Vielfachschichtsysteme}

Um den inneren Aufbau und die Periodizität der Schichtpakete genauer zu untersuchen, wurden röntgendiffraktometrische Messungen durchgeführt.

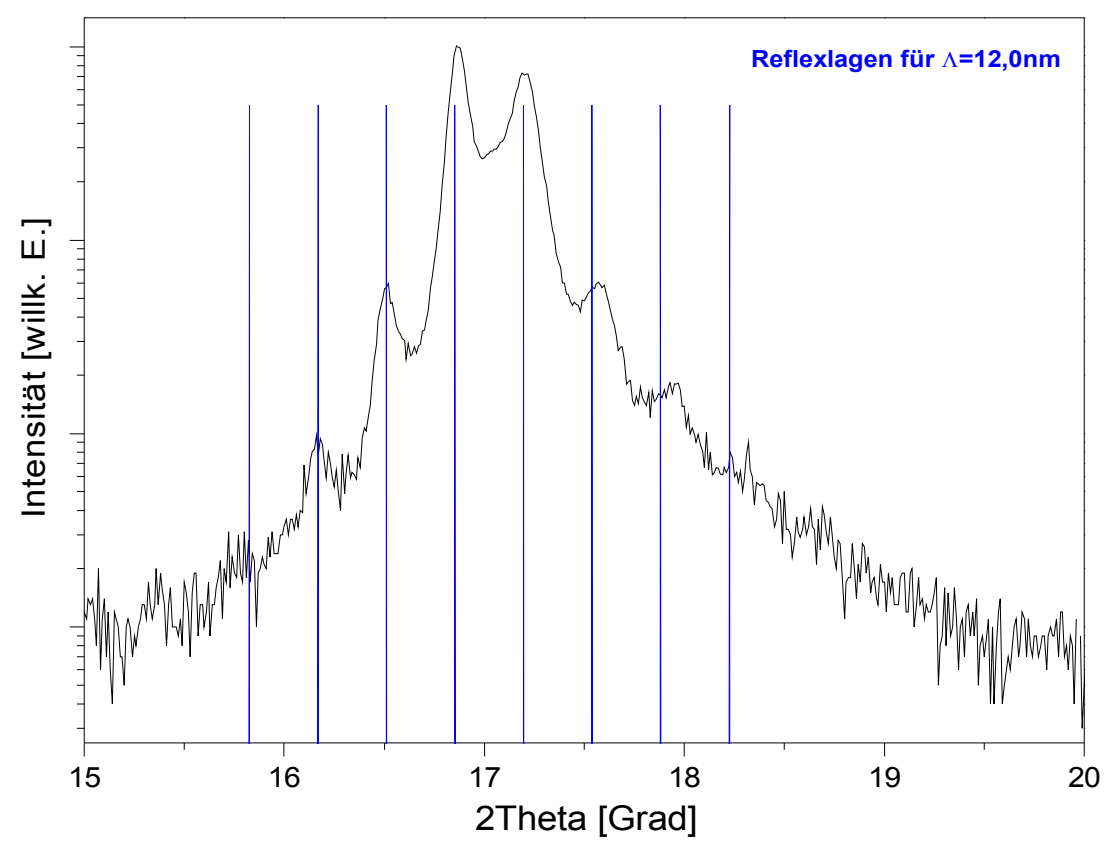

Abbildung 93: XRD-Spektrum eines Schichtsystems 50x(2nm Fe/10nm Au) auf glattem m-plane Saphir, wie hergestellt, $\lambda_{X R D}=0,709494 \AA$.

Abbildung 93 zeigt beispielhaft ein Diffraktogramm eines 50x(2nm Fe/10nm Au) Schichtsystems auf glattem m-plane $\mathrm{Al}_{2} \mathrm{O}_{3}$ mit Positionen der Reflexe des künstlichen Übergitters für die Übergitterperiodizität von $\Lambda=12,0 \mathrm{~nm}$. Die Analyse zeigt, dass die gewünschte Übergitterperiode bei der Deposition mit hoher Genauigkeit erreicht werden konnte.

Ausschlaggebend für die Sichtbarkeit der Überstrukturanteile im Diffraktogramm ist eine ausreichende Kohärenztiefe, d.h. parallel zur Substratnormalen muss Intensität von vielen Grenzflächen rückgestreut werden. Defekte wie Korngrenzen, Zwillingsgrenzen etc. verhindern, dass Intensität aus einer ausreichenden Informationstiefe in den Detektor gelangt, um die künstliche Übergitterstruktur abzubilden. 


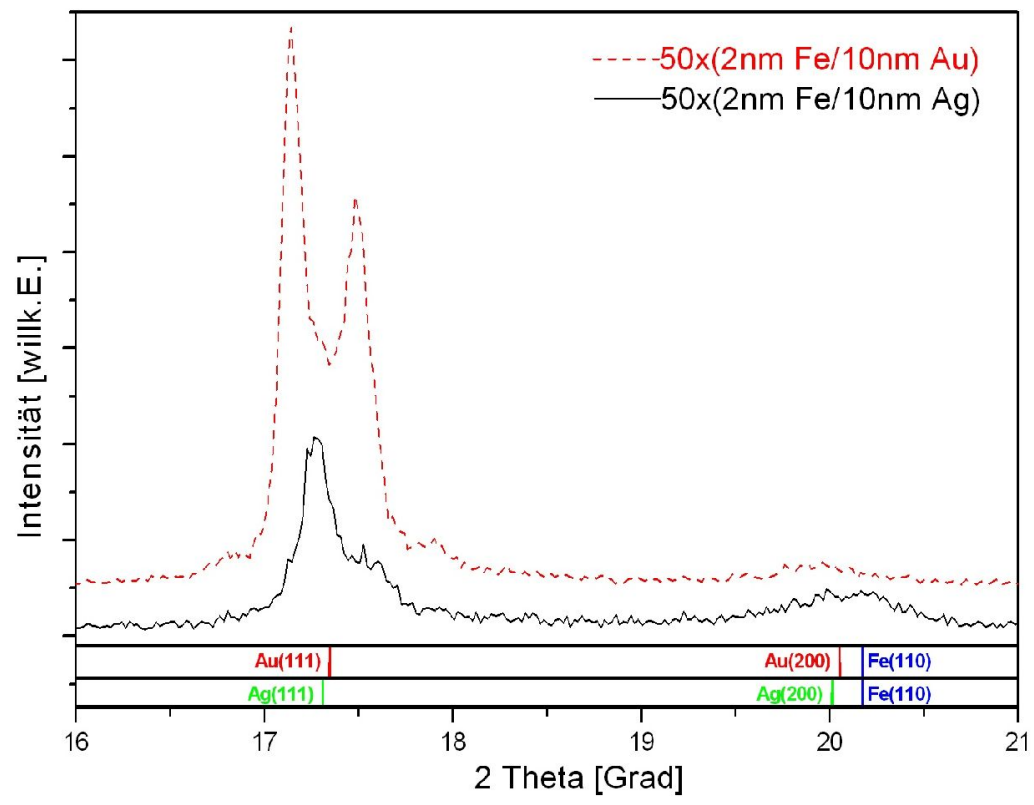

Abbildung 94: XRD: Vergleich der Schichtsystemen 50x(2nm Fe/10nm Au) und $50 x(2 \mathrm{nmFe} / 10 \mathrm{~nm} \mathrm{Ag})$ auf facettiertem Substrat.

Ein Vergleich von Schichtsystemen gleicher Einzelschichtdicke und Gesamtlagenzahl der Komponenten Eisen/Silber und Eisen/Gold (Abbildung 94) zeigt deutlich, dass ein wesentlich kohärenteres Wachstum im System Eisen/Gold vorliegen muss. Die Ausbildung von Überstrukturreflexen im Großwinkelbereich ist in diesem System deutlich ausgeprägter und kann nicht durch den größeren Streufaktor des Golds gegenüber Silber erklärt werden. Um weitere Strukturuntersuchungen auch an diesen Schichtensystemen, die aufgrund ihrer mikrostrukturellen Beschaffenheit und durch die facettierte Unterlage nur stark reduzierte Überstrukturen im Großwinkelbereich zeigen, durchführen zu können, erfolgten Messungen mit wesentlich höherer Brillianz der Strahlungsquelle am Hamburger Synchrotronstrahlungslabor HASYLAB.

Der Einfluss der Facettierung des Substrat auf die Intensität wird in Abbildung 95 deutlich. Dargestellt sind Diffraktogramme von drei im gleichen Beschichtungsprozess hergestellten Schichtsystemen mit 50x(2nm Fe/10nm Au). Die unteren beiden Diffraktogramme zeigen Daten des Vielfachschichtsystems auf facettiertem m-plane $\mathrm{Al}_{2} \mathrm{O}_{3}$-Substrat. Dabei unterscheiden sich die Orientierungen bei der Herstellung in vertikal (für präferentielle Materialverteilung) und horizontal (für eine homogene Materialverteilung). Die obere Kurve resultiert von Messungen des Systems auf einem glatten m-plane $\mathrm{Al}_{2} \mathrm{O}_{3}$-Substrat. Zur besseren Vergleichbarkeit mussten die Kurven des Schichtsystems auf dem facettiertem Substrat zehnfach überhöht dargestellt werden, da es aufgrund der für die Messungen ungünstigen Oberflächengeometrie kommt es zu einem schlechteren Signalzu-Rauschen-Verhältnis kommt. Die Facettierung der Unterlage hat aber auch einen Einfluss auf das Wachstum und somit auf die Mikrostruktur des deponierten Schichtsystems. Insgesamt ist die Intensität in den Reflexen rund um den (111)-Kristallreflex von Gold reduziert. Bei den unteren Kurven ist deutlich ein (200)-Anteil bei $2 \Theta=20^{\circ}$ erkennbar, auf dem glatten Substrat finden sich Körner dieser Orientierung nicht. Die Intensitätsverteilung ist dort breit, so dass dort geringe Anteile von Eisen in (110)-Orientierung vorhanden sein könnten. In keinem Diffraktogramm war es jedoch möglich, eindeutig einen Eisenreflex zu identifizieren. Die Schichten wachsen bezüglich der Edelmetallkomponente stark (111)-orientiert auf den Facettenseiten auf, diese Orientierung ist entsprechend um den Facettierungswinkel von ca. 17,6 gegen die Normalen der Gesamtsubstrat- 
fläche verkippt. Folglich erfüllt nur eine kleiner Anteil die Bragg-Bedingung.

Ein Vergleich der Psi-Scans um die Normalrichtung der Substrate beim Glanzwinkel für die (111)-Orientierung (Abbildung 96), verdeutlicht dies. Die Daten auf facettiertem Substrat sind doppelt überhöht dargestellt. Während im Fall des glatten Substrat die Intensität im Zentrum um die Oberflächennormale gebündelt $\left(\mathrm{FWHM}=3,4^{\circ}\right)$ ist, findet man bei den facettierten Substraten eine breite Verteilung. Im Fall der präferentiellen Bedeckung (vertikal, facettiert) ist ein ausgeprägtes Maximum bei $-20,5^{\circ}$ sichtbar $\left(\mathrm{FWHM}=3,5^{\circ}\right), \quad$ zusätzlich ein lokales Maximum um $0^{\circ}$ und ein sehr schwaches im Bereich der zweiten Facettenseite von ca $16^{\circ}$. Im Fall der homogeneren Deposition, bei horizontaler Ausrichtung der Facettierungsrichtung bei der Deposition, sind die Intensitätsunterschiede $z W$

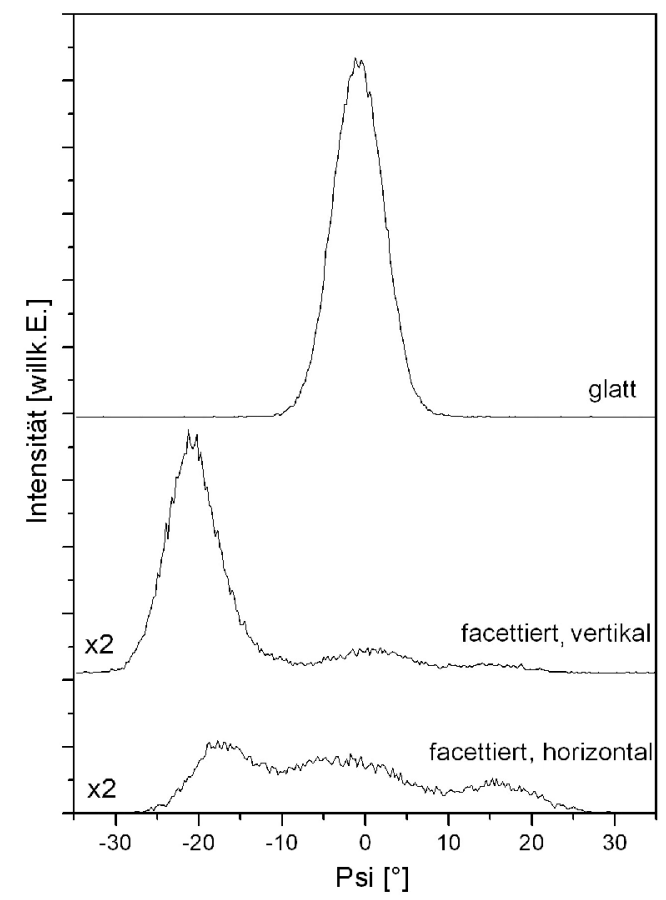

Abbildung 96: Vergleich von Psi-scans bei der Au(111)-Reflexbedingung System: $50 x(2 \mathrm{~nm} \mathrm{Fe} / 10 \mathrm{~nm}$ Au), facettierte Substrate doppelt überhöht.

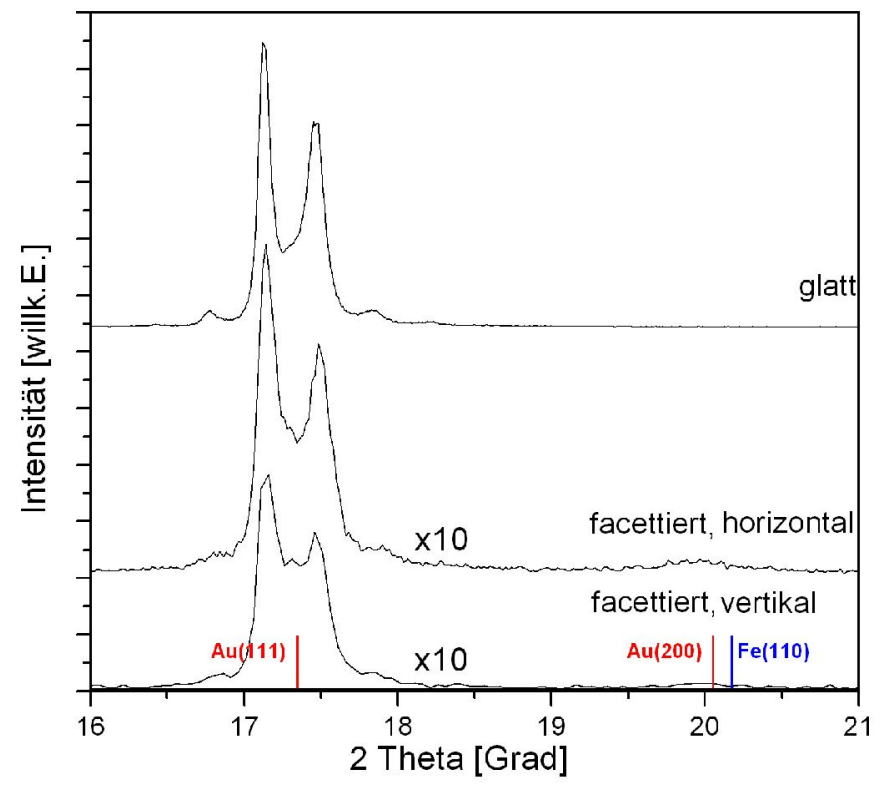

Abbildung 95: XRD: Einfluss der Substratorientierung und -beschaffenheit, System: $50 x(2 \mathrm{~nm} \mathrm{Fel} \mathrm{10nm} \mathrm{Au);} \mathrm{die} \mathrm{Daten} \mathrm{für}$ die facettierten Substrate sind zehnfach überhöht dargestellt.

zwischen diesen Maxima geringer. Die Reflexe sind breit, was $\mathrm{zu}$ einem insgesamt erhöhten Intensitätsbereich zwischen $-20^{\circ}$ und $20^{\circ}$ führt. Für die Lage der Maxima können hier $-17,6^{\circ},-2^{\circ}$ und $16,3^{\circ}$ ermittelt werden. Die integrale Intensität ist für die Psi-Scans auf facettiertem Substrat mit 7000 (horizontal) und 8432 (vertikal) vergleichbar, die auf glattem Substrat liegt bei 20408. Für die vertikal eingebauten Substrate wird deutlich, dass das (111)Wachstum in Richtung der Facettennormalen von Gold auf der Seite, die der Goldquelle abgewandt ist gegenüber der der Quelle zugewandten Seite verschlechtert ist. Dabei ist jedoch aufgrund der geometrischen Verhältnisse (siehe Abbildung 97) und aufgrund der Tatsache, dass es sich beim Magnetronsputtern um ein nur schwach gerichtetes Verfahren handelt, nicht davon auszugehen, dass die Intensitätsverhältnisse zwischen den Peaks bei $-20,5^{\circ}$ und $16^{\circ}$ dem Verhältnis an deponiertem Gold auf den unterschiedlichen Facettenseiten entsprechen. Vielmehr ist anzunehmen, dass nur ein kleiner Teil der Goldkörner, die auf der abgewandten Seite aufwachsen, (111)-orientiert ist. Dies wird unterstützt durch die - im Gegensatz zur Probe auf glattem Substrat - klar messbare Intensität im Bereich des $\mathrm{Au}(200)$-Reflexes (vgl. Abbildung 95). Es ist bekannt, dass die Depositionsrichtung, d.h. der Winkel zwischen Materialstrom und Substratnormalen, einen wesentlichen Einfluss auf die Mikrostruktur von gesputterten Filmen hat [Abe 97], [Har 88]. Einen weiteren Einfluss des Depositionswinkels auf das Wachstum erkennt man auch an der Position des 
absoluten Maximums in diesem Psi-Scan. Mit 20,5 $5^{\circ}$ ist dieses etwas weiter gegenüber der Substratnormalen verkippt als der mittlere

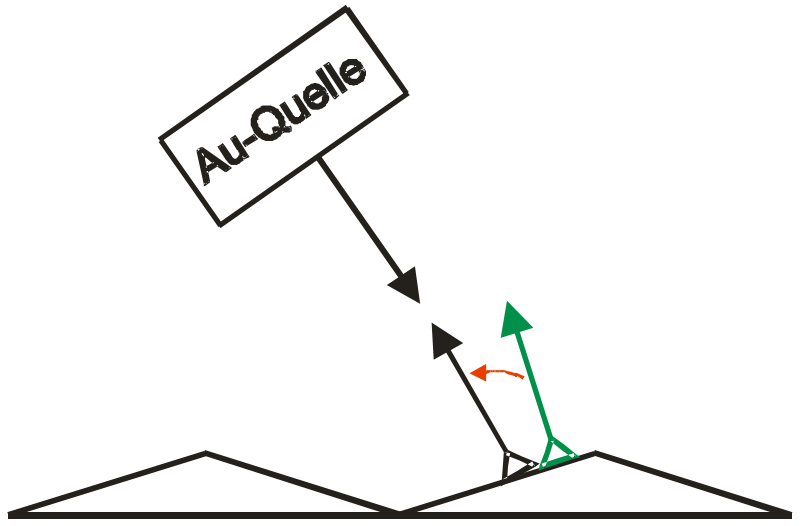

Abbildung 97:Verkippung der (111)-Richtung in Richtung des Materialstroms. Facettierungswinkel. Dies kann auf den quellenzugewandten Seiten der Verkippung der (111)-Richtung der Körner zum Materialstrom hin zugeschrieben werden (Abbildung 97).

Auf dem zur Beschichtung horizontal eingebauten Substrat findet man in Abbildung 96 die Maxima mit $-17,6^{\circ}$ und $16,3^{\circ}$ bei den Winkeln, die sehr gut dem Facettierungswinkel der Oberfläche entsprechen.

Der Vergleich zeigt aber vor allem deutlich, dass mit drastischen Intensitätseinbußen durch die Facettierung der Oberfläche zu rechnen ist. Zwar kann durch eine gezielte Verkippung der Probe eine Messung im absoluten Maximum erfolgen, dennoch ist auch dort die absolute

Intensität geringer.

Die Facettierung hat aber nicht nur Auswirkung auf die Kristallstruktur und das Gefüge der Edelmetallschichten, sondern auch auf den Betrag und die Güte der Periodizität des künstlichen Übergitters. Als Folge davon sind bei den Schichtsystemen auf den facettierten Substraten in Abbildung 94 deutlich weniger Satellitenreflexe um die Lage der Kristallreflexe zu erkennen. Größere Rauigkeiten der Grenzflächen zwischen Eisen und Edelmetall, aber auch eine Variation in der Schichtdicke über den untersuchten Probenbereich, führen zu einer Verschlechterung der BraggBeugung am Übergitter und damit $\mathrm{zu}$ einer Minderung des Verhältnisses zwischen Spitzen und Tälern (peak-to-valley Verhältnis, PV) (siehe Abbildung 98).

Tabelle 2 gibt die Werte für die Intensitätsverhältnisse der beiden Hauptmaxima zum dazwischengelegenen Tal wieder.

\begin{tabular}{|l|r|r|}
\hline & \multicolumn{1}{|c|}{ PV1 } & \multicolumn{1}{c|}{ PV2 } \\
\hline glatt & 3,8 & 2,7 \\
\hline horizontal & 2,5 & 1,8 \\
\hline vertikal & 1,7 & 1,3 \\
\hline
\end{tabular}

Tabelle 2: PV-Verhältnisse der Eisen/Gold-Schichtsysteme.

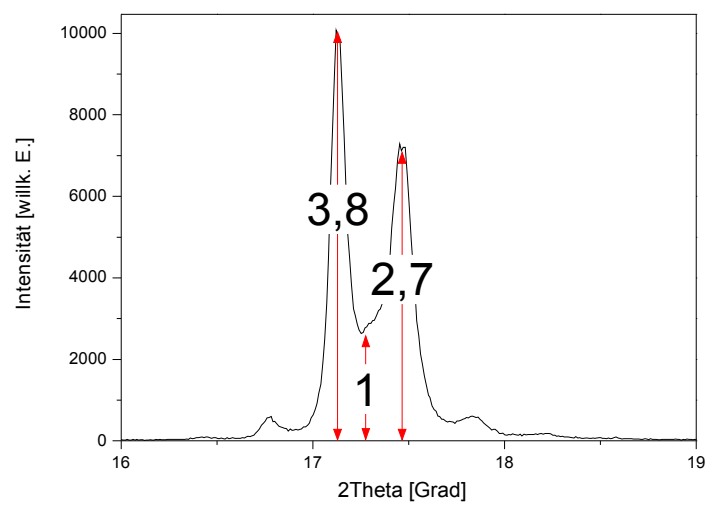

Abbildung 98: XRD: Erläuterung des PVVerhältnisses.

Die PV-Werte zeigen das geringste Verhältnis für die Proben, die vertikal eingebaut wurden. Dies ist verständlich, da in diesem Fall die Variation in der Schichtdicke einer Komponente auf der Breite einer Facettenseite besonders groß ist (siehe Abbildung 97). Hingegen sind die Variationen der Einzelschichtdicken im Fall der horizontalen Ausrichtung der Facetten bei der Deposition die gleichen wie auch bei glatten Substraten, da beide Quellen und die Facettierungsrichtung in einer Ebene liegen. Nahezu alle getroffenen Aussagen sind übertragbar auf das System Eisen/Silber. Vergleicht man die Systeme unterschiedlicher Edelmetallkomponenten Gold (Abbildung 95) bzw. Silber (Abbildung 99), sind zwei Details besonders auffällig. Zum einen ist die Ausbildung von Satellitenreflexen um den (111)-Kristallreflex im Eisen/Silber geringer als im Eisen/Gold. Der Anteil, den die Intensität aus der Übergitterstrukturbeugung bei der Faltung hat, ist in diesem System deutlich geringer. Die Lage des absoluten Intensitätsmaximums entspricht im Eisen/Silber 
schon vor einer Temperung besser der idealen Lage, d.h. dem Gitterabstand der (111)-Ebenen des Silbers im Gleichgewicht.

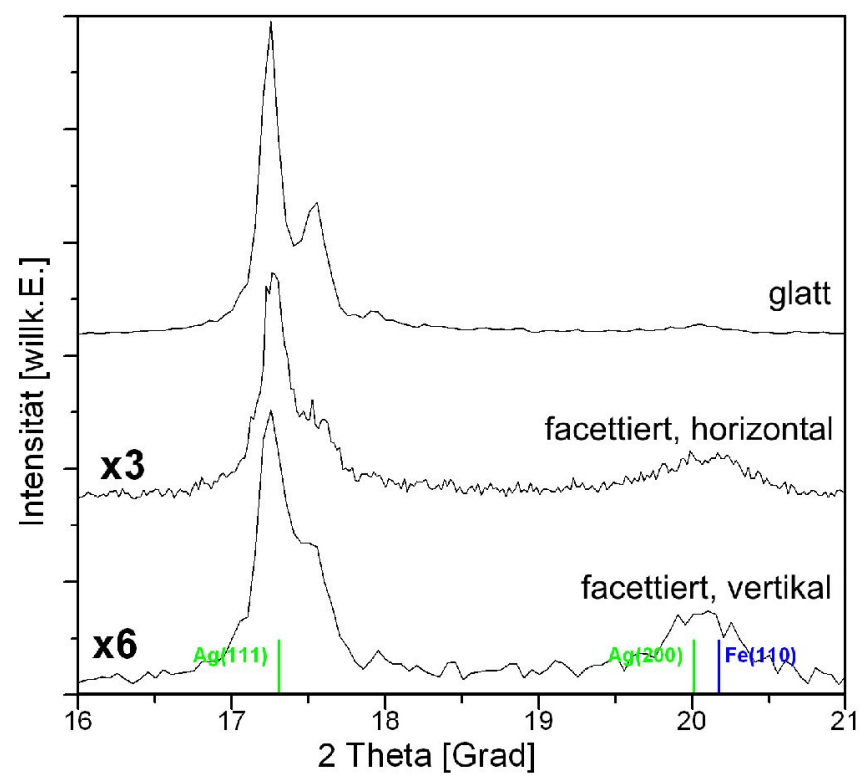

Abbildung 99: XRD: Einfluss der Substratorientierung und -beschaffenheit, System: 50x(2nm Fe/10nm Ag), wie hergestellt (auffacettiertem Substrat dreifach bzw. sechsfach überhöht).

Des weiteren zeigt sich im System Eisen/Silber eine schlechtere Texturierung. Schon auf dem glatten Substrat ist ein Gold-(200)-Anteil deutlich erkennbar, der bei Schichten auf vertikal eingebauten Substraten auf eine Intensitätsverhältnis von (200) zu (111) von 0,29 anwächst.

Zusammenfassend kann gesagt werden, dass die Substratfacettierung zu einer sehr starken Reduktion in der gemessenen Intensität in den Diffraktogrammen führt. Die charakteristische Satellitenstruktur in den Großwinkelspektren bestätigt die angestrebte Periodizitätslänge von 12nm. Die Systeme zeigen bevorzugtes (111)-Wachstum der Edelmetallebenen parallel zur lokalen Oberfläche. Die Ersetzung von Gold durch Silber führt neben einer starken Reduktion der Gesamtintensität auch zu einer verringerten Kohärenz des Übergitters einhergehend mit einem größeren Fehlorientierungsanteil. Die Beschichtungsgeometrie beeinflusst neben der Materialverteilung auch das Wachstum. Insgesamt ergeben sich für die weiteren Analysen zwei Anforderungen:

1. Die Facettierung des Substrats erfordert Untersuchungen, die über eine nur parallel zur Gesamtoberflächennormalen sensitiven Methode hinausgeht und durch Verkippung bzw. Drehung der Probe Aussagen zur orientierungsabhängigen Kornverteilung zulässt.

2. Zur temperaturabhängigen Analyse von Proben auf facettierten Substraten, insbesondere der Eisen/Silber-Schichtsysteme in Bragg-Brentano-Geometrie ist eine intensive Strahlungsquelle notwendig. 


\subsubsection{Texturanalyse der Vielfachschichtsysteme}

Um den Einfluss der Substratfacettierung auf die Kornverteilung und Orientierung $\mathrm{zu}$ analysieren, wurden ferner Terturanalysen der Schichtpakete vorgenommen. Da die Schichtpakete eine (111)-Vorzugsorientierung in Richtung der Substrat- bzw. Facettennormalen bezüglich des Edelmetalles aufweisen, eignet sich diese Reflexbedingung gut, anhand von Texturanalysen die Auswirkungen der facettierten Unterlage auf die Orientierungsverteilung zu untersuchen.

Der geringe Unterschied des Gitterabstands der Gold (200)-Ebenen mit 2,03915 $\AA$ (bzw. Silber (200)-Ebenen

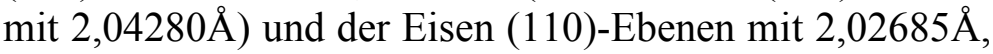
sowie die Reflexverbreiterung erschweren jedoch eine Analyse und Trennung für die Orientierung der Eisenkörner in den Schichtsystemen. Hinzu kommt die schlechte Röntgensichtbarkeit von Eisen durch den kleinen Atomformfaktor.

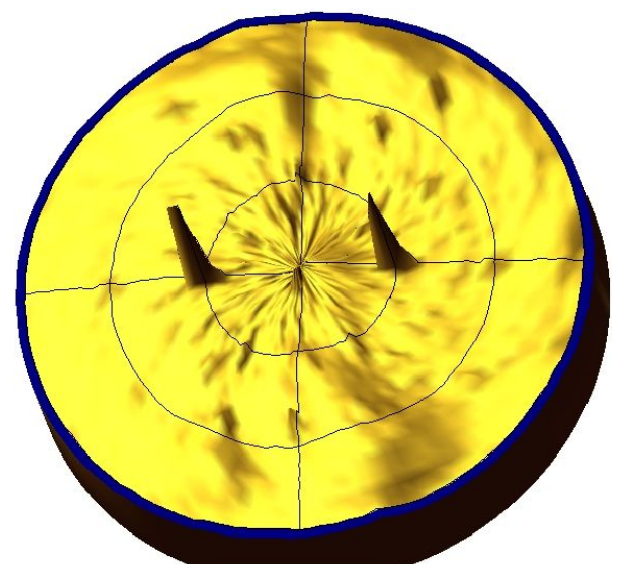

Abbildung 100: Texturmessung eines unbeschichteten facettierten $\mathrm{Al}_{2} \mathrm{O}_{3}$-Substrats.

Um den Einfluss des Substrats auf die Texturmessungen feststellen zu können, wurde eine Referenzmessung eines unbeschichteten Substrats unter den Bedingungen für (111)-Polfiguren für Silber bzw. Gold vorgenommen. In Abbildung 100 sind zwei Reflexe zu erkennen, die unter einem Winkel von $\mathrm{psi}=30^{\circ}$ zur Substratnormalen unter einem Winkel von phi $=180^{\circ}$ zueinander angeordnet sind. Durch Kenntnis der Kristallstruktur des Substrat, der Reflexbedingung und der in dieser Halbraum-Darstellung zweizähligen Symmetrie lassen sich diese eindeutig den $(11 \overline{2} 0)$ - bzw. $(2 \overline{1} \overline{10})$-Ebenen (a-plane) zuordnen. Der Abstand der Ebenen beträgt 2,3794 $\AA$ [JCPDS 46-1212] und liegt nahe an denen der Gold- bzw.

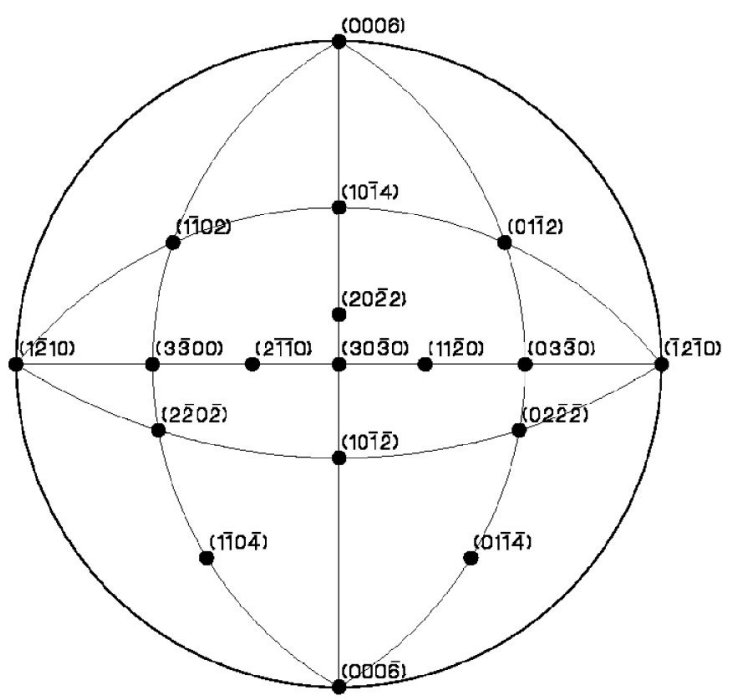

Abbildung 101: Standardprojektion für $\alpha-\mathrm{Al}_{2} \mathrm{O}_{3}$ in ( $10 \overline{1} 0)$-Orientierung (aus [Ost 03]).
Silber-(111)-Netzebenenabständen von $2,35882 \AA$, so dass diese Reflexe in den entsprechenden Texturmessungen sichtbar sind.

Abbildung 101 zeigt die stereographische Projektion von $\mathrm{Al}_{2} \mathrm{O}_{3}$ in Richtung der m-plane (10 $0 \overline{1} 0)$-Orientierung [Ost 03]. Hieraus wird klar, dass unter einem Winkel von $30^{\circ}$ mit zweizähliger Symmetrie die (11 $\overline{2} 0)$ - bzw. (2 $\overline{1} 10)$-Richtungen $\mathrm{zu}$ finden sind. Die Projektion zeigt außerdem, dass die Facettenflächen, die $(20 \overline{2} 2)$-, aber auch die $(10 \overline{1} \overline{2})$-Flächen, gerade unter einem Winkel von $90^{\circ} \mathrm{zu}$ den a-plane Flächen in der Ebene der Substratnormalen (10 10$)$ liegen. Daher entspricht die Verbindungslinie zwischen den $(11 \overline{2} 0)$ - bzw. (2 $\overline{1} \overline{1} 0)$ - Substratreflexen der Facettierungsrichtung. 


\section{Auf glatten Substraten}

Die Texturanalysen von Schichtsystemen aus 50x(2nm Fe/10nm Au) (Abbildung 102a) bzw. 50x (2nm Fe/10nm Ag) (Abbildung 102b) zeigen die Drahttextur dieser Filme. Neben dem sehr starken zentralen Peak zeigt vor allem die linke Polfigur des Gold-Schichtsystems einen Ring hoher Intensität unter einem Winkel von $70,5^{\circ}$. Dies entspricht dem kleinsten Winkel zwischen den unterschiedlichen (111)-Ebenen. Die ringförmige Verteilung zeigt, dass keine Vorzugsorientierung in der Filmebene gegeben ist. Die zuvor beschriebene Substratsignatur der $(11 \overline{2} 0)$-Flächen ist ebenfalls zu sehen.
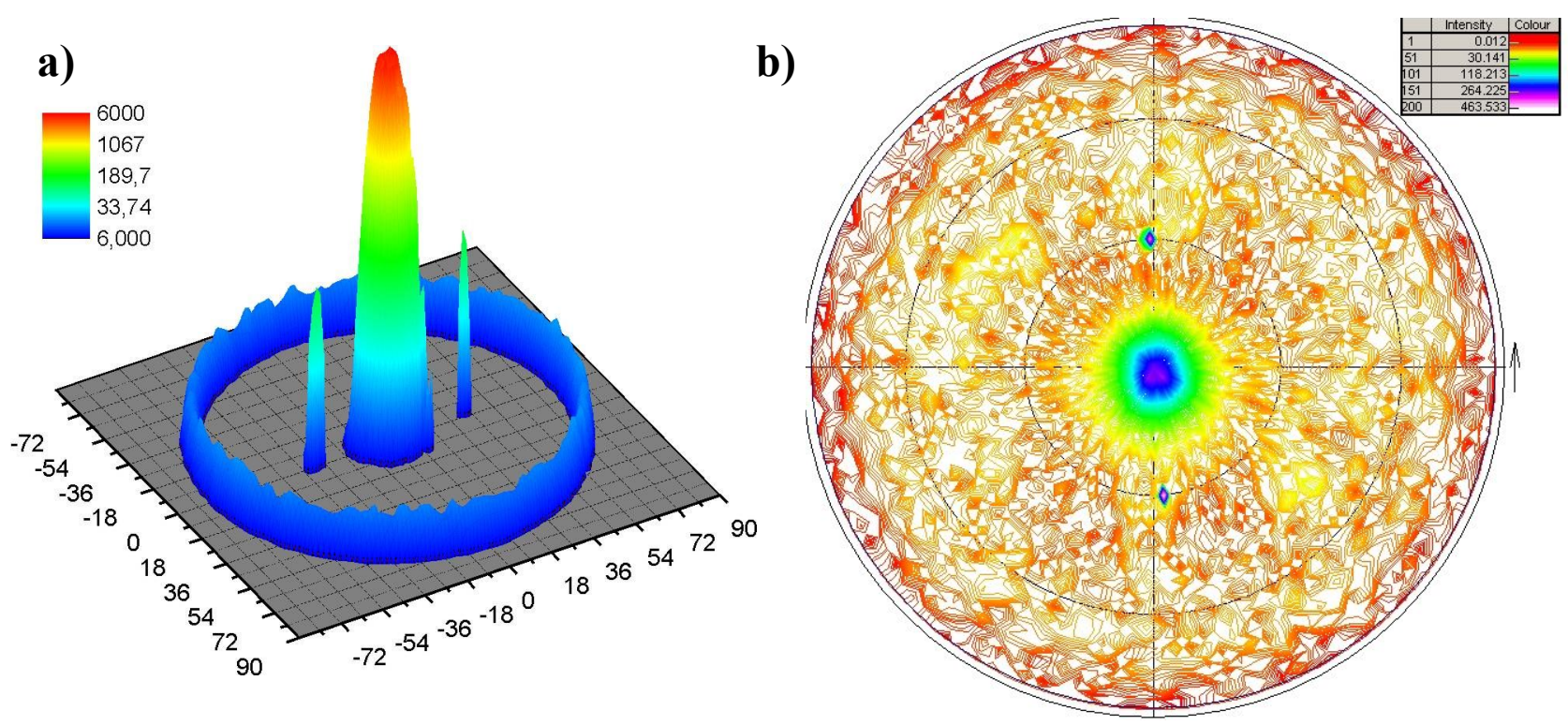

Abbildung 102: (111)-Polfigur a) eines 50x(2nm Fe/10nm Au)-Schichtsystems, dreidimensional und b) eines $50 x(2 \mathrm{~nm} \mathrm{Fe} / 10 \mathrm{~nm} \mathrm{Ag})$-Schichtsystems, zweidimensional auf glattem $\mathrm{Al}_{2} \mathrm{O}_{3}$.

Abbildung 102b verdeutlicht in der zweidimensional projizierten Darstellung die Textur des Eisen/Silber-Schichtpakets. Der zentrale Reflex der (111)-Polfigur ist deutlich schwächer und der Ring der zugehörigen (111)-Ebenen unter $70,5^{\circ}$ verschwindet fast vollständig. Es wird aber auch hier keine signifikante Häufung bei speziellen Phi-Winkeln unter Psi=70, $5^{\circ}$ beobachtet. Wäre eine Texturierung in der Filmebene vorhanden, würde man eine dreizählige Symmetrie erwarten, d.h. bei Psi $=70,5^{\circ}$ Maxima in der Intensität auf dem Phi-Kreis im Abstand von $120^{\circ}$.

Der (111)-Orientierung der Schichtpakete parallel zur Substratnormalen entsprechend zeigen (200)-Polfiguren einen Ring unter 54,7 $7^{\circ}$ (nicht dargestellt). Dies entspricht dem kleinsten Winkel zwischen den Ebenen dieses Typs. Die sehr gleichmäßige Intensitätsverteilung auf dem Ring bestätigt, dass keine in-plane Orientierung der Schichten vorliegt. Eine aufgrund der niedrigen Substrattemperaturen ohnehin unwahrscheinliche Epitaxie zum $\mathrm{Al}_{2} \mathrm{O}_{3}$-Substrat kann dementsprechend ebenfalls ausgeschlossen werden.

Die Texturmessungen konnten also zeigen, dass die (111)-Netzebenen der Edelmetallkomponenten in den Schichtsystemen parallel zur Substratoberfläche wachsen, bezüglich der Orientierung in der Filmebene jedoch statistisch verteilt sind. 


\section{Auf facettiertem Saphir}

Die Halbraum-Darstellung in Abbildung 103 macht den Einfluss der Substratfacettierung auf die Eisen/GoldSchichtpakete besonders deutlich. Die in Abbildung 102 beobachtete maximale Intensität im Zentrum teilt sich in zwei aus dem Zentrum verkippte Maxima auf. Die ringförmige Intensitätsverteilung bei $70,5^{\circ}$ ist ebenfalls in zwei Anteile geteilt und in derselben Ebene verkippt. Die Drehung erfolgt dabei um eine Achse, die durch die Verbindungslinie der Substratreflexe gegeben ist, also die Facettierungsrichtung.

Die Skizze in Abbildung 104 ver- facettiertem $\mathrm{Al}_{2} \mathrm{O}_{3}$.

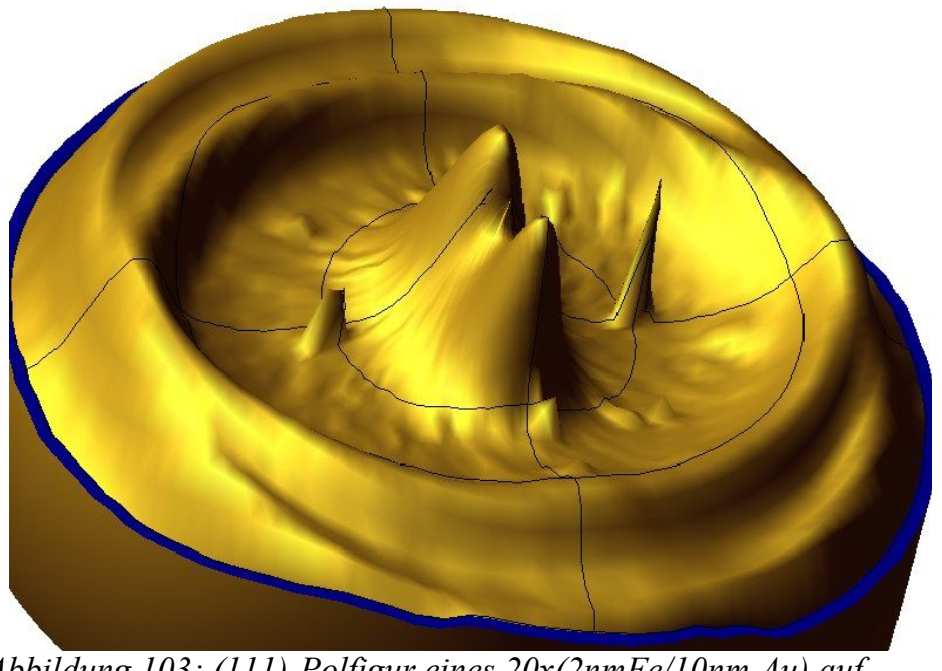

anschaulicht das generelle Aufwachsverhalten des Schichtsystems auf den

Facetten. Die dichtest gepackten (111)-Ebenen wachsen parallel zu den Facettenflächen, so dass der Winkel zwischen den Maxima in den (111)-Polfiguren (Abbildung 103) gerade dem Winkel zwischen den beiden Facetten entspricht.

Während bei der Deposition des in Abbildung 103 analysierten Schichtsystems die Verteilung der beiden Elemente gleichwertig auf beide Facettenseiten erfolgte, führt der Einbau der Substrate mit Facettierungsrichtung senkrecht zur Verbindungslinie zwischen den Quellen dazu, dass eine präferentielle Verteilung von Eisen bzw. Gold auf den unterschiedlichen Facetten entsteht.

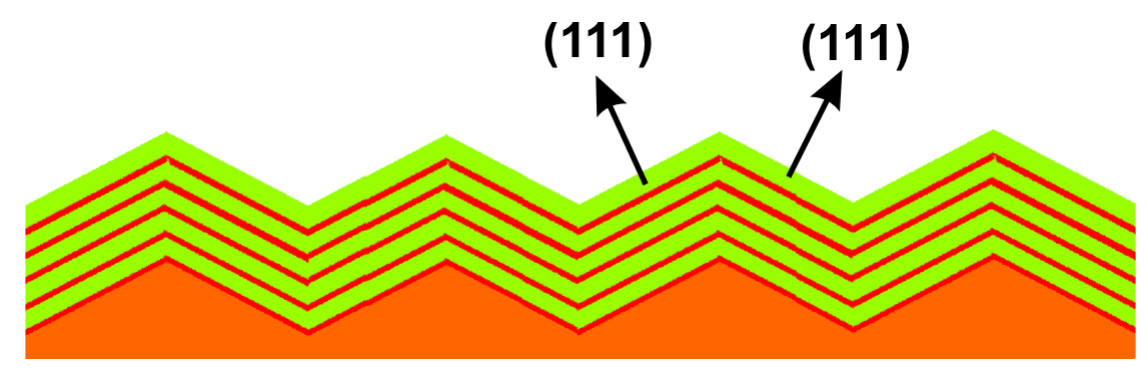

Abbildung 104: Vereinfachtes Bild zum Vielfachschichtaufbau auf dem facettierten Substrat. (111)-Ebenennormalen sind parallel zu den Facettenseitennormalen.

Die Intensitätsverteilung im Zentrum der (111)-Polfiguren (Abbildung 105) ist dementsprechend modifiziert. Man findet ein absolutes Maximum auf der Facettenseite, die der Edelmetallquelle zugewandt ist $\left(\mathrm{Psi}=-22^{\circ}\right)$. Das zur zweiten Facette korrespondierende, schwächere Maximum liegt bei Psi $=18^{\circ}$, aber auch im Zentrum ist noch eine hohe Intensität zu beobachten. Diese Winkel stimmen sehr gut mit denen mittels AFM bestimmten Werten für die Facettierungswinkel von $18-23^{\circ}$ überein. In einem Kreis von $70,5^{\circ}$ um das absolute Maximum ist, somit ebenfalls um $22^{\circ}$ verkippt, die Intensitätserhöhung durch die anderen (111)-Ebenen zu sehen. Bei der (111)-Polfigur des Eisen/Silber-Schichtsystems (Abbildung 105b) ist fast ausschließlich die zentrale Intensität von einer, der Silber-Quelle zugewandten Seite sichtbar. Gleiches gilt für den zugehörigen Ring bei $70,5^{\circ}$. 
Genau auf dem Phikreis bei Psi $=30^{\circ}$ sind in Abbildung 105 die bereits in der Hintergrundmessung

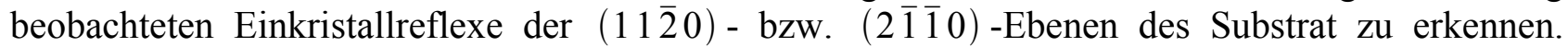
Diese liegen genau um Phi $=90^{\circ}$ gedreht zu den Maxima der Facetten, d.h. hier bestätigt sich, dass die Facettierungsrichtung in $[11 \overline{2} 0]$-Richtung verläuft. Der Winkel von $18^{\circ}$ entspricht der theoretischen Lage der Saphir- $(20 \overline{2} 2)$-Ebene mit 17, $6^{\circ}$ zur $(10 \overline{1} 0)$-Ebene sehr gut, die andere von Heffelfinger et al. vorgeschlagene Facettenseite, die (10 122$)$-Kristallfläche, läge unter $32,4^{\circ}$ zur (1010) -Fläche [Hef 97]. Dieser Winkel wird nicht erreicht, d.h. die Facettenseite entspricht nicht der Kristallebene, sondern ist, wie in Kapitel 3 schon beschrieben, aus Stufen mit dieser Kristallfläche aufgebaut.
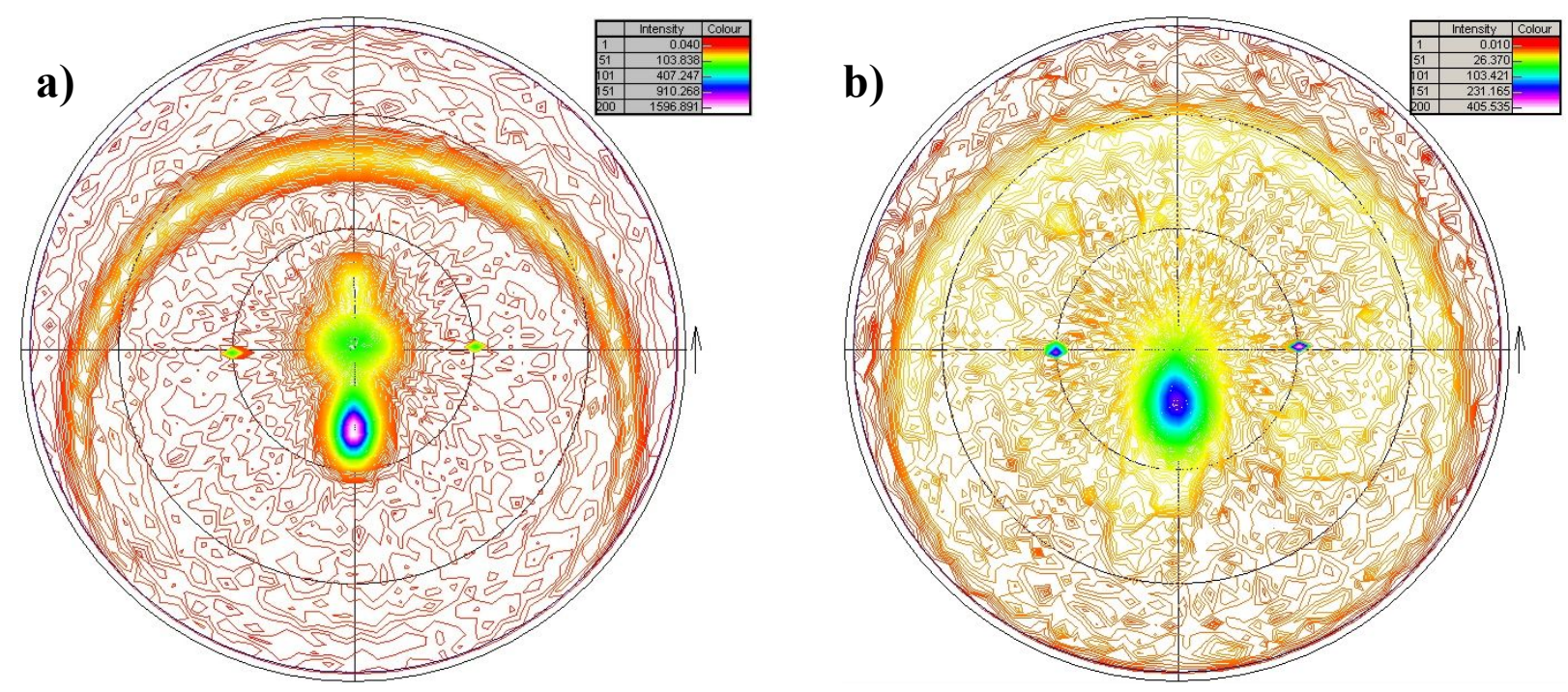

Abbildung 105: (111)-Polfigur a) eines 50x(2nm Eisen/10nm Gold)-Schichtsystems bzw.

b) $50 x\left(2 \mathrm{~nm}\right.$ Eisen/10nm Silber)-Schichtsystems (b) auf facettiertem, vertikal beschichtetem $\mathrm{Al}_{2} \mathrm{O}_{3}$.

Der Psi-Scan (Abbildung 96) hatte gezeigt, dass trotz homogener Deposition die Ausprägung der Maxima auf beiden Facettenseiten unterschiedlich ist. Die (111)-Polfigur in Abbildung 147b (Seite 123) bestätigt diese Diskrepanz. Hier zeigt sich wiederum die Auswirkung der Substratmorphologie auf den Schichtaufbau, denn die unterschiedliche Oberflächenbeschaffenheit der Facettenseiten führt hier zu einer stärkeren Störung der Kristallstruktur auf der nanofacettierten Seite. Ein Vergleich mit Abbildung 103 zeigt außerdem, dass die Unterschiede in der Höhe der Maxima der beiden Facettenflächen mit steigender Doppellagenzahl wachsen. Kumulativen Rauigkeits- bzw. Störungsentwicklung (siehe Kapitel 5.1.5) könne dazu beitragen wider.

Die Röntgenanalysen, sowohl in Bragg-Brentano Geometrie als auch die Texturanalysen zeigen für die Schichtsysteme mit Silber niedrigere Intensitäten und breitere Reflexe. Hierzu trägt neben dem geringere Formfaktor für Silber auch der Abbau innerer Spannungen aus Gitterfehlpassungen zwischen Eisen und dem Edelmetall bei. Silber bildet, bedingt durch die sehr niedrige Stapelfehlerenergie von $\gamma_{\mathrm{Ag}}=20 \mathrm{~mJ} / \mathrm{m}^{2}\left(\gamma_{\mathrm{Au}}=40 \mathrm{~mJ} / \mathrm{m}^{2}\right)$ [Haa 94], leicht Zwillingsgrenzen, wodurch die Röntgenkohärenz gestört wird. Die starke Neigung zur Zwillingsbildung wurde auch bei TEMUntersuchungen an Eisen/Silber-Schichtsystemen auf glatten Substraten beobachtet [Her 99]. Im System Eisen/Gold werden Verspannungen aus der Fehlpassung zwischen Eisen und Gold ebenfalls im Wesentlichen von der Edelmetallkomponente aufgenommen, (da die Schermodulen von Eisen mit $11,07 \cdot 10^{10} \mathrm{~N} / \mathrm{m}^{2}$ und Gold $4,23 \cdot 10^{10} \mathrm{~N} / \mathrm{m}^{2}$ stark differieren), jedoch führt hier im Wesentlichen eine Krümmung der Grenzflächen zur Relaxation der Spannungen.

Die Texturmessungen konnten aber zeigen, dass eine (111)-Drahttextur, d.h. eine Ausrichtung der (111)-Ebenennormalen der Edelmetallkomponente parallel zur Substratnormalen bei Deposition auf 
glattem Substrat vorliegt und die Orientierung dieser Körner in der Ebene statistisch ist. Auf den facettierten Substraten wächst die Schicht in der Gestalt auf, dass die (111)-Ebenen parallel zu den Facettenflächen liegen, also dichtestet gepackt auf der lokalen Oberfläche aufwachsen.

\subsubsection{Einfluss der Substratfacettierung und der Facettenoberfläche auf die Mikrostruktur der Vielfachschichtsysteme}

Um die Auswirkung lokal ausgezeichneter Punkte wie die eines einzelnen Facettendachs auf das Wachstum des deponierten Schichtsystems analysieren zu können, wurden TEMQuerschnittspräparationen auf facettiertem $\mathrm{Al}_{2} \mathrm{O}_{3}$ erstellt. Die globalen Ergebnisse der Streumethoden lassen sich damit durch lokale aufgelöste Aussagen der konventionellen und hochauflösenden Elektronenmikroskopie vervollständigen. Korrelationseffekte beim Wachstum der Vielfachschichtsysteme auf der facettierten Substratoberfläche sind somit auch identifizierbar, wenn sie nicht charakteristisch für weite Bereiche der Schicht sind, sondern auf ausgezeichnete Orte beschränkt sind.

Daher wurden Querschnittspräparationen von Schichtpaketen auf glattem bzw. auf facettiertem Saphir angefertigt. Hierbei ist die Präparation sehr problematisch, da die sehr unterschiedliche Härte von Schicht- und Substratmaterial einen gleichmäßigen Abtrag bei allen Schritten der Präparation vor allem aber auch beim letzten, dem Ionendünnen verhindert. Das Sektorätzen, bei dem das weichere Schichtmaterial durch das Substrat geschützt wird, näher erläutert z.B. von Hartung [Har 96], ist dies unabdingbare Voraussetzung, aber noch bei weitem kein Garant zur erfolgreichen Präparation. Die mangelnde Haftung des Films am Substrat ist ein weiterer wesentlicher Faktor, der trotz vorheriger, intensiver Reinigung der

Substrate zu vielen Fehlschlägen führte.

Abbildung 106 zeigt einen Substratbereich im Querschnitt, in dem die Schicht vollständig von Substrat abgelöst ist. Es zeigt sich die weitestgehend symmetrische Facettierung der Saphiroberfläche. Außerdem sind auf den linken Facettenseiten Stufen erkennbar, wohingegen die rechten glatt sind. Die Periodizitätslänge beträgt ca. $280 \mathrm{~nm}$, bei einer Facettierungshöhe (Spitze $\mathrm{zu}$ Tal) von ca. $40 \mathrm{~nm}$.

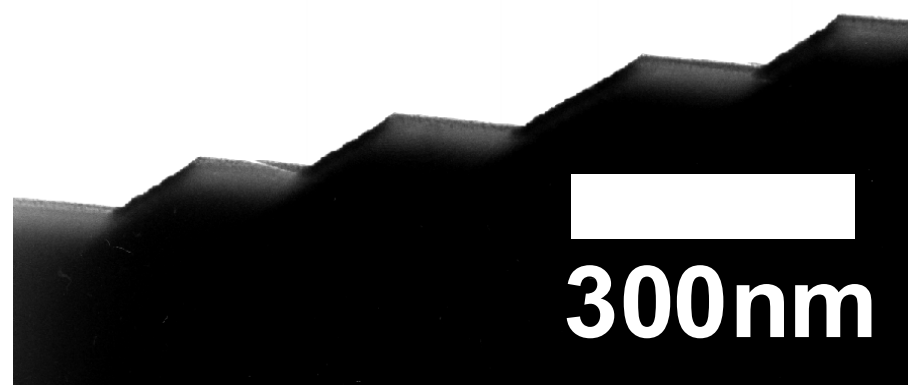

Abbildung 106: TEM-Querschnitt: Facettierung des $\mathrm{Al}_{2} \mathrm{O}_{3}$-Substrat in regelmäßige, gleichseitige Facetten.

\section{Eisen/Silber-Schichtsysteme}

Diese ausgeprägte Oberflächenmorphologie des Substrat bleibt über das gesamte Schichtsystem erhalten. Abbildung 107 zeigt eine Querschnittsaufnahme eines Eisen/Silber-Systems und bestätigt damit die Aussagen aus den rasterkraftmikroskopischen Analysen (vgl. Abbildung 78), dass die Substratfacettierung vollständig, präzise und ohne wesentliche Ausglättung an der Schichtoberseite nach 50 Doppellagen mit einer Gesamtschichtdicke von 600nm erhalten ist. Die beiden Grenzlinien Schichtsystem-Vakuum stellen sich als weitgehend parallel zur Grenzlinie Substrat-Schichtsystem. Lediglich die Facettenkämme wirken an der Oberseite leicht abgerundet. Nicht nur die Facetten 
werden durch das Schichtsystem hindurch an die Schichtoberseite abgebildet, sondern auch Fehler in der Facettierung und kleinere Oberflächenmerkmale.

Von Interesse ist, ob die Extrema der facettierten Unterlage auf die Mikrostruktur des Schichtsystems lokal einen Einfluss haben.

Abbildung 108 zeigt als Hellfeldbild eine Übersicht

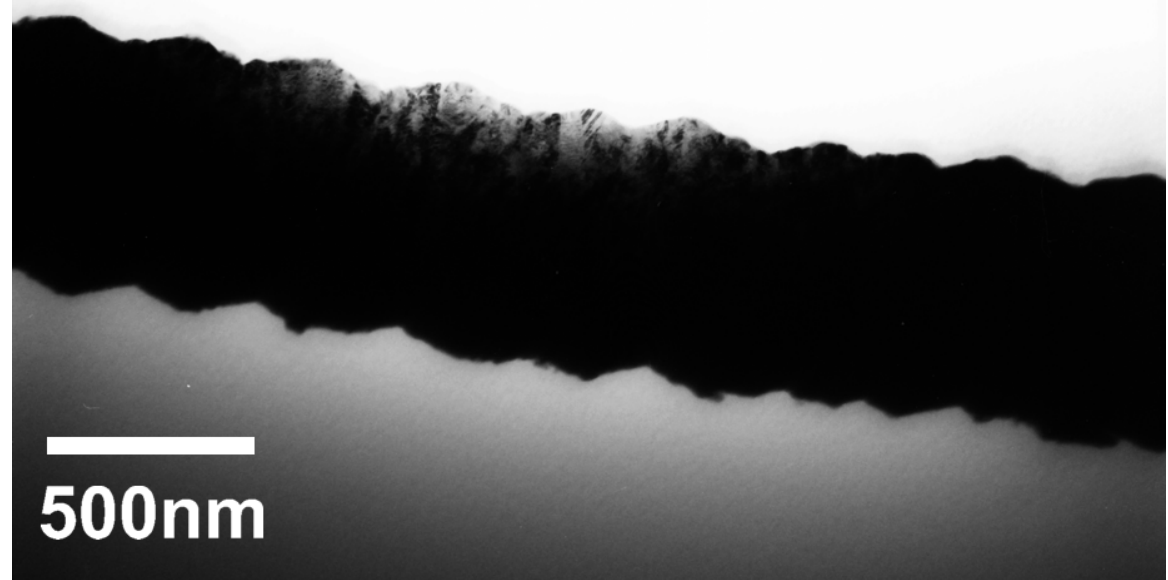
über einen substratnahen

Abbildung 107: TEM-Querschnitt eine 50x(2nmFe/10nm Ag) Schichtpakets auf facettiertem $\mathrm{Al}_{2} \mathrm{O}_{3}$.

Bereich des Schichtpakets. In dieser Darstellung zeigt sich ein feinkörniges Gefüge des Systems in der ersten und zweiten Doppellage. Die erste Eisenlage ist nicht klar von der ersten Silberlage zu trennen. Erst mit der zweiten Eisenlage wird der Schichtaufbau deutlich. Dies ist konsistent mit dem durch die Leitwertmessungen ermittelten Inselwachstum von Eisen und der Perkolation in der ersten Edelmetallschicht. In den folgenden, sehr dünnen Bereichen sind die unterschiedlichen Lagen von Silber und Eisen am besten zu unterscheiden. Die Verlaufsrichtung der inneren Grenzflächen ist vorwiegend parallel zur Grenzfläche Substrat-Schichtsystem. Die auftretenden Kontrastunterschiede haben unterschiedliche Ursachen (Spannungen, Massenzahl, Dicke, etc.). Zur Diskussion der Entwicklung des Grenzflächenverlaufs in steigender Lagenzahl ist eine Reduktion des Kontrastes auf einen chemischen Kontrast wünschenswert.

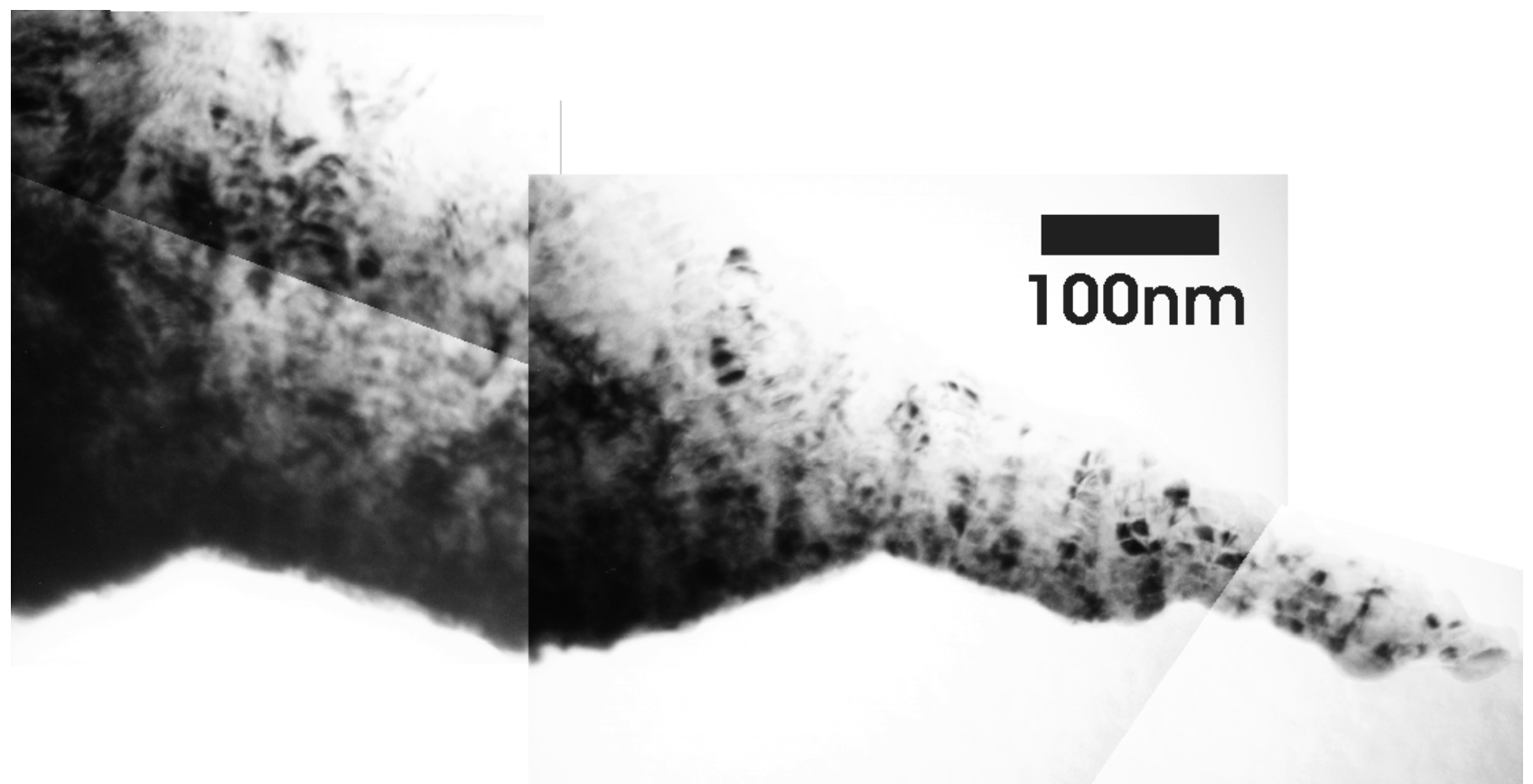

Abbildung 108: Querschnittspräparation TEM: Hellfeld-Abbildung eines 50x(2nm Eisen/10nm Silber)-Schichtsystems auffacettiertem $\mathrm{Al}_{2} \mathrm{O}_{3}$. 


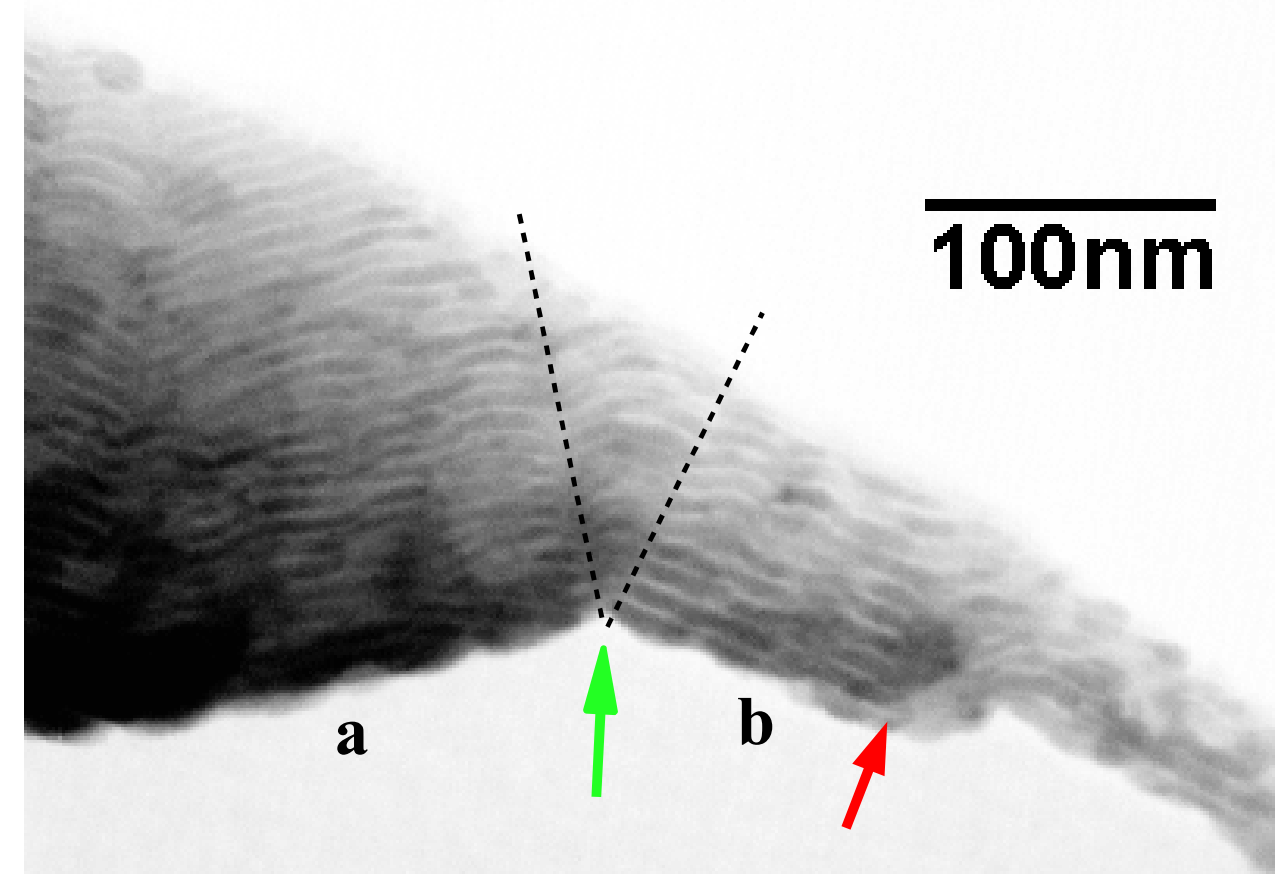

Abbildung 109: STEM-Querschnitt, inverse Darstellung. Dunkle Bereiche entsprechen Silber, helle Eisen. Der grüner Pfeil markiert eine Facettenspitze, der rote Pfeil ein Facettental. a) nanofacettierte und b) glatte (10 111$)$-Facettenseite.

Eine solche massenzahlsensitive Darstellung mittels STEM ist in Abbildung 109 gezeigt. Um die Sichtbarkeit zu verbessern, wurde die inverse Darstellung gewählt, so dass helle Bereiche eisenreichen und dunkle silberreichen entsprechen. In dieser Darstellung ist es möglich, nachzuvollziehen, wie sich die momentane Oberfläche während der Deposition entwickelt hat, da die inneren Grenzflächen über die gesamte Schichtdicke sichtbar sind. Auf der Facettenseite (b) ist nach den ersten 4-5 Doppellagen die Ausbildung von Wachstumssäulen mit gekrümmten Grenzflächen zu beobachten, wie sie schon auf glatten Substraten nachgewiesen wurden [Her 99]. Jedoch dominiert hier diese Krümmung die resultierende Querschnittsstruktur bei weitem nicht so stark.

An der durch einen grünen Pfeil gekennzeichneten Facettenspitze zeigt sich jedoch eine besondere Beeinflussung des Wachstums. Von der ersten Lage an bildet sich ein Keil aus, der durch eine besonders starke Krümmung der Grenzfläche auffällt. Durch diesen Keil wird der Winkel zwischen den Ebenennormalen der Facettenseiten geschlossen. Das Wachstum auf den Facettenseiten bleibt auf diese Weise weitgehend unbeeinträchtigt von dem Extremum der Facettenspitze.

Dahingegen erscheint die Vielfachschichtperiodizität im Bereich der Facettentäler stark gestört, der Verlauf der hellen Eisen-Schichten ist in diesem Bereich nicht durchgängig erkennbar (roter Pfeil).

Auf der breiten Facettenseite (a) in Abbildung 109 wächst das System zunächst vergleichsweise ungeordnet und es ist eine Welligkeit kleiner Wellenlängen von ca. 20nm erkennbar. Die Doppelschichtdicke erscheint in diesem Bereich auch noch starken Schwankungen unterworfen zu sein, bedingt durch unterschiedliche Wachstumsbedingungen. Eine Ursache für diesen ungeordneten Anfangsbereich liegt möglicherweise in der gestuften Beschaffenheit der Facettenseite. Wie schon im Kapitel 3.2 erläutert, ist der Aufbau der Facettenseiten unterschiedlich. Während die eine Seite sehr glatt ist, wird bei der anderen Seite der Symmetriewinkel nur durch Bildung einer Vielzahl von Stufen erreicht. Eben diese Nanofacetten sind bevorzugte Nukleationszentren und fördern das Wachstum ausgehend von diesen Keimstellen. Eine genauere Analyse dessen folgt am Beispielsystem Kohlenstoff/Kobalt (Seite 103).

Folglich ist das Schichtsystem auf dieser Facettenseite in den ersten Lagen durch die heterogene 
Nukleation an diesen kleinen Ausgleichsstufen geprägt. Erst mit zunehmender Schichtdicke wird auf dieser großen Facettenseite ein vergleichsweise glatter Verlauf der Grenzflächen und eine gleichmäßige Doppelschichtdicke erkennbar.

Auf der benachbarten Facettenseite (b) in Abbildung 109 sind die Grenzflächen schon in Substratnähe glatt. Der andere Facettenseitentyp bildet sehr glatte (10 $\overline{1} 1)$-Kristallflächen aus, was zu einer geringen Rauigkeit in den ersten Lagen des Schichtsystems auf dieser Facettenseite führt.

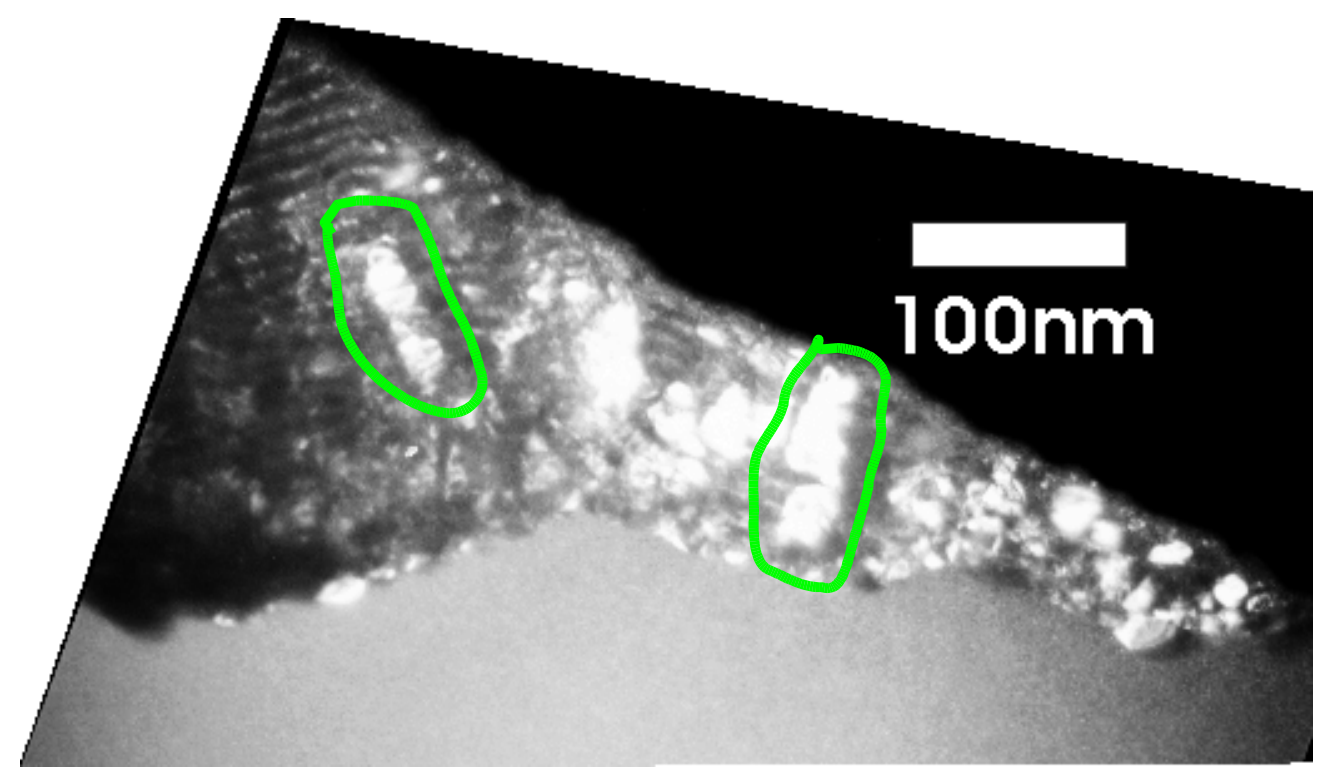

Abbildung 110: Querschnittspräparation TEM Dunkelfeld-Abbildung eines 50x(2nm Eisen/10nm Silber)-Schichtsystems auf facettiertem $\mathrm{Al}_{2} \mathrm{O}_{3}$.

In einer Dunkelfeldabbildung liefern Körner gleicher Orientierung (der Zonenachse bzgl. des Elektronenstrahls) gleichen Kontrast. Abbildung 110 zeigt mehrere, parallel zu den lokalen Facettennormalen ausgedehnte helle Bereiche, die entsprechend gleiche Orientierungen aufweisen. Zwei Säulen, die sich über ca. 10 Doppellagen erstrecken, sind grün markiert. Sie verdeutlichen, dass das Wachstum senkrecht zur Facettenoberfläche auf beiden Facettenseiten stattfindet. Die Breite der hell kontrastierten Bereiche ist aber erheblich schmaler als die Breite einer Wachstumssäule, die durch die Krümmung der inneren Grenzflächen begrenzt wird. Das bestätigt, dass mehrere verschieden orientierte Körner in Eisen/Silber-Schichtsystemen in einer Wachstumssäule nebeneinander vorliegen. 


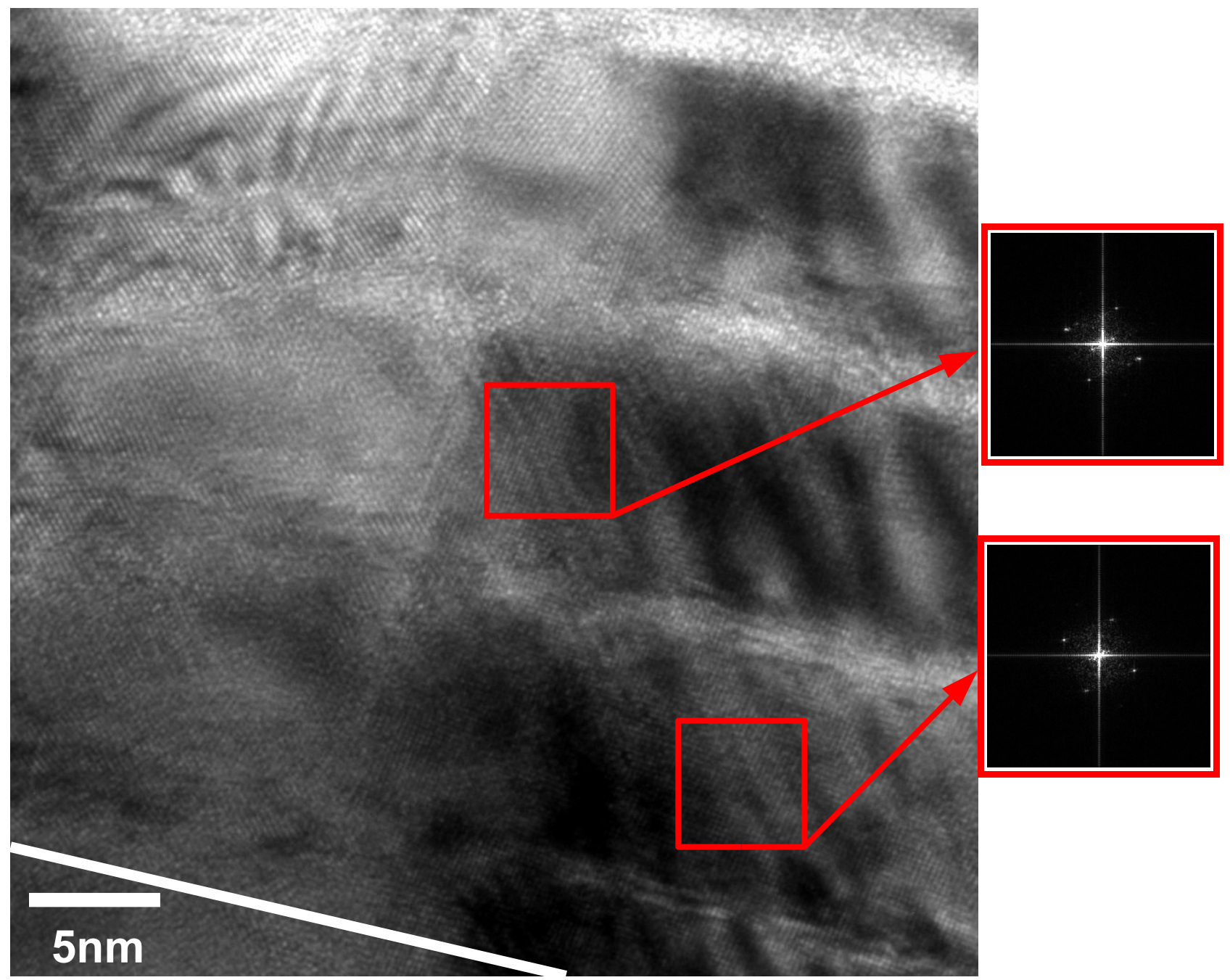

Abbildung 111: Querschnittspräparation HRTEM oberhalb glatter Facettenfläche mit Ausschnitts-FourierTransformationen. Die weiße Linie deutet den Verlauf der Facettenseite an.

Die unterschiedlich hohe Defektdichte an verschiedenen Bereichen der Schicht wird durch einen Vergleich deutlich. Diese Hochauflösungsbilder Abbildung 111 und Abbildung 112 zeigen gleich große Ausschnitte, zum einen von einem Bereich oberhalb einer Facettenfläche zum anderen oberhalb eines Tals. Das Gefüge oberhalb einer Facettenfläche (Abbildung 111) besteht aus großen Körnern, zu erkennen an den gleich strukturierten Bereichen der typischen Fringes (Beugungsmuster). In der rechten Bildhälfte sind die Einzellagen deutlich unterscheidbar. Die Grenzflächen verlaufen nahezu in horizontaler Richtung. In der Bildmitte ist gut erkennbar, dass ein Strukturübertrag über die Eisenlage hinweg erfolgt, da die Silberschichten ober- und unterhalb dieselben Beugungsstruktur aufweisen. Dies bestätigt die Vermutung, dass analog zu den Untersuchungen am System Eisen/Gold [Bor 02a] eine strukturvermittelnde Beziehung zwischen Eisen und Silber (Nishiyama-Wassermann) zu epitaktischem Wachstum in den Wachstumssäulen über viele Doppellagen hinweg führen kann.

Abbildung 112 zeigt dahingegen eine andere Mikrostruktur. Viele, sehr kleine Bereiche verschiedener Beugungsmuster sind zu erkennen und eine Unterscheidung unterschiedlicher Einzellagen ist nicht möglich. Die Abbildung zeigt einen Bereich oberhalb eines Facettentals (zur Position siehe Skizze Abbildung 113). In diesem Bereich kommt es durch die parallel zu den Facettenseitennormalen wachsenden Körner zu einer sehr defektreichen Zone. Immer wieder treffen Körner von benachbarten Facettenseiten aufeinander. Die Folge sind mikrostrukturell stark gestörte 


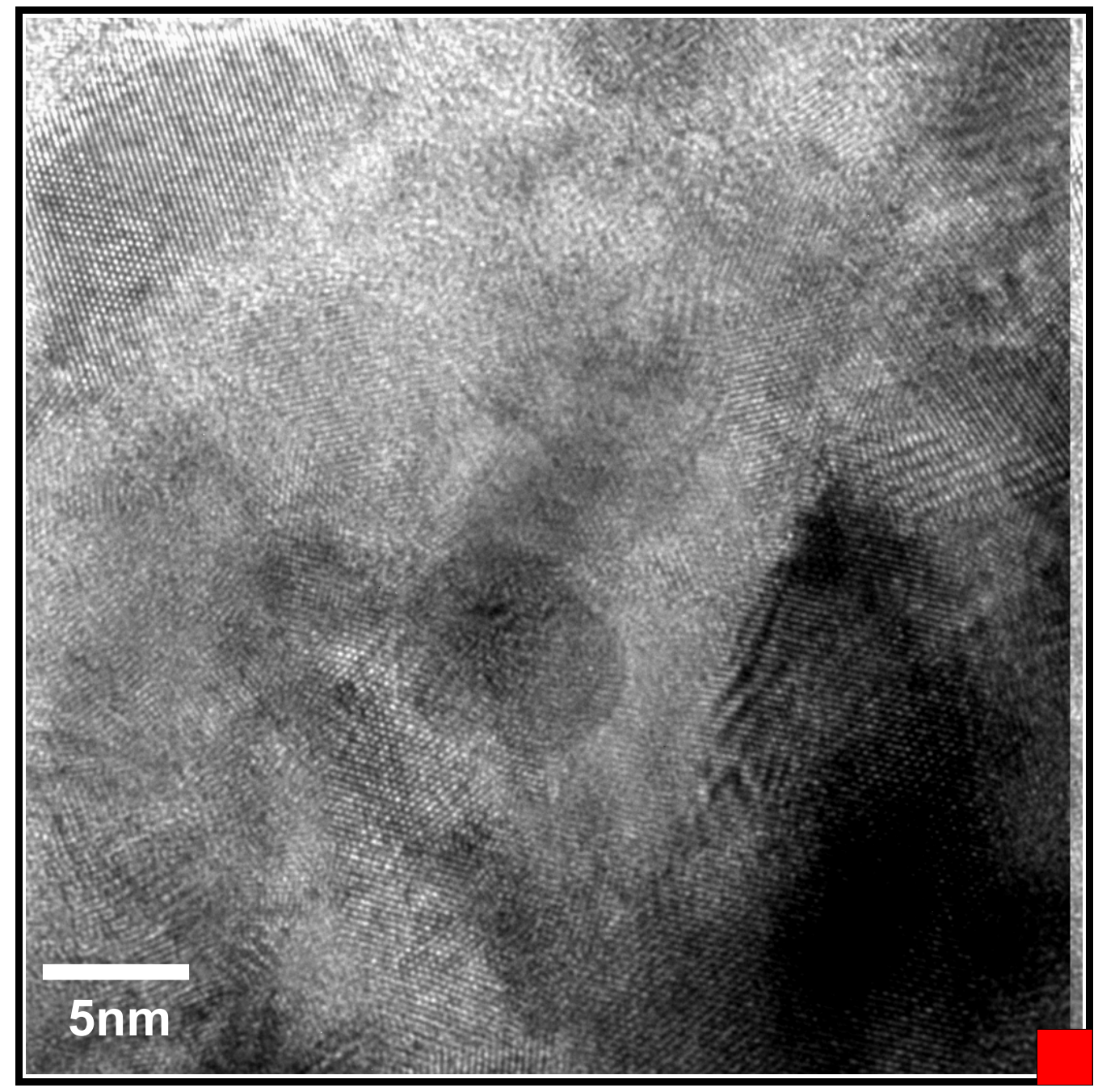

Abbildung 112: Querschnittspräparation HRTEM über einem Facettental.

Bereiche oberhalb der Facettentäler, in denen die Schichtabfolge nicht mehr erkennbar ist und die von einem nanokristallinen Gefüge geprägt sind. Die für Silber typische Zwillingsbildung ist ebenfalls häufig zu finden, die niedrige Stapelfehlerenergie führt auch in Nanopartikel und -röhren zu starker Zwillingsbldung [Hof 02], [Mor 01].

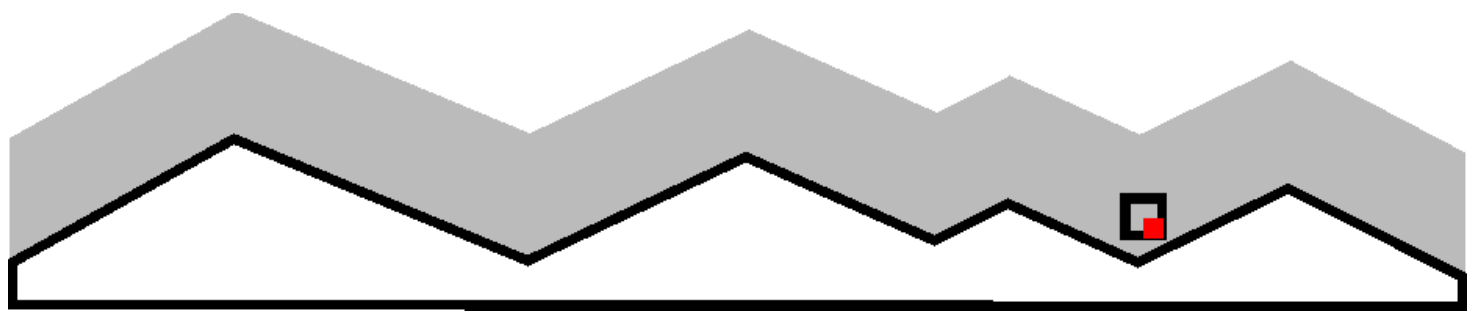

Abbildung 113: Position der HRTEM-Abbildung 112 über einem Facettental.

Die ortsaufgelösten Untersuchungen mittels TEM an Querschnittsproben vervollständigen also das Bild von der Mikrostruktur der metallischen Schichtsysteme, das sich aus dem Wachstumsverhalten und den Untersuchungen durch Streumethoden ergeben hat. Es kann folgendermaßen zusammengefasst werden: 
Die inneren Grenzflächen im Schichtsystem verlaufen im Wesentlichen parallel zu den Facettenseiten und das kolumnare Wachstum findet mit der (111)-Richtung parallel zur Normalen auf den Facettenseiten statt. HRTEM zeigt den epitaktischen Strukturübertrag benachbarter Silberschichten über die Eisenzwischenschicht. Krümmungen der Grenzflächen, wie sie bei Deposition entsprechender Schichtsysteme auf glatten Substraten bekannt sind, sind sichtbar. Die Substratmorphologie erlaubt jedoch eine Relaxation von Spannungen an den Facettenkämmen. Hier zeigt das System eine verstärkte Krümmung der Grenzflächen in einem Keil, der den Winkel zwischen den Facetten ausfüllt. Oberhalb von Facettentälern ist der Aufbau und das Korngefüge stark gestört.

Die unterschiedliche Güte des Aufbaus des Schichtsystems auf den unterschiedlichen Facettenseiten deutet auf eine Beeinflussung durch die Beschaffenheit der Facettenseite hin. Im System EisenSilber ist dieser Einfluss aber nur schwer von anderen, die Mikrostruktur störenden Einflüsse, wie Fehlpassungsspannungen zu trennen.

\section{Kohlenstoff/Kobalt - Kumulative Wachstumseffekte}

Die Untersuchungen an Kohlenstoff/Kobalt-Schichtsystemen auf glatten Substraten zeigten, dass sie sich durch sehr glatte Grenzflächen auszeichnen. Die in diesem System geringen, vorliegenden Grenzflächenspannungen ermöglichen ein ideales Wachstum mit geringer Rauigkeit. Aus diesem Grund eignet sich das System besonders, die Auswirkungen einer facettierten Unterlage auf das Wachstum und die Entwicklung von Störungen im Verlauf einer steigenden Doppellagenzahl zu studieren. Im Folgenden werden daher Ergebnisse der Querschnittspräparation von Schichtsystemen aus 30 Doppellagen 2nm Kobalt/10nm Kohlenstoff auf facettierter $\mathrm{AbO}_{3}$-Oberfläche dargestellt und diskutiert.

Abbildung 114 und Abbildung 115 zeigen Übersichts-TEM-Analysen eines solchen Schichtpakets im Querschnitt. Dunkler Kontrast zeigt die Kobaltlagen, heller die Kohlenstofflagen. Die Bilder verdeutlichen, dass die Facettierung des Substrats über das gesamte Schichtpaket hinweg erhalten bleibt.

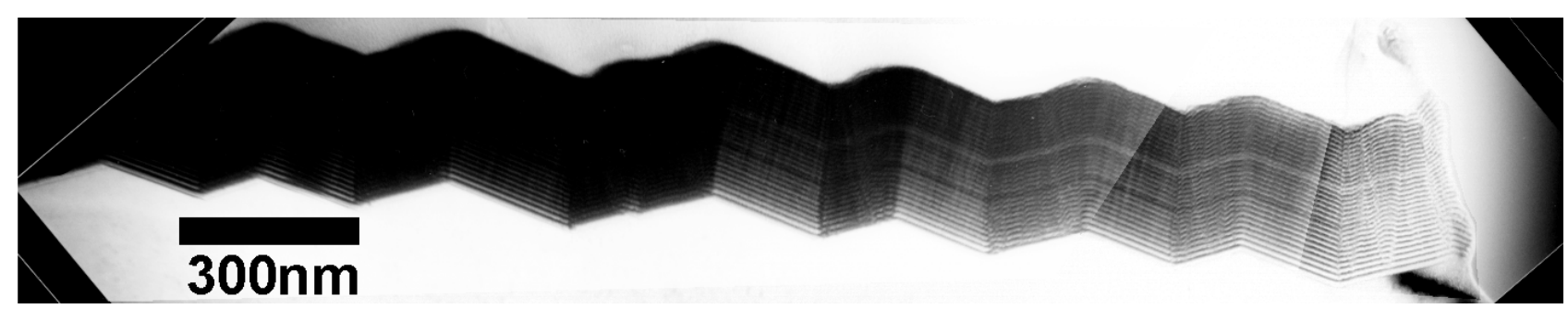

Abbildung 114: TEM-Querschnittsanalyse: 30x(2nm Kobalt/10nm Kohlenstoff) auffacettiertem $\mathrm{Al}_{2} \mathrm{O}_{3}$, dunkel Kobalt, hell Kohlenstoff.

Wie im Eisen/Silber- bzw. Eisen/Gold-System ist der Verlauf der Einzelschichten parallel zum Substrat und an der Oberseite des Schichtpakets wird die Substratmorphologie sehr präzise abgebildet. Die geringe Oberflächenspannung des Kohlenstoffs sowie der regellose Aufbau verhindern, dass Spannungen durch Gitterfehlpassungen entstehen. Entsprechend ist auch das Wachstumsverhalten verändert und der Schichtaufbau ist regulärer mit der Folge, dass der Transfer der Substratfacettierung in diesem System deutlich besser ist.

Der im Vergleich zu den Systemen aus Eisen und Silber bzw. Eisen und Gold hohe Massenunterschied der Komponenten führt zu einem deutlich besseren chemischen Kontrast in der TEM-Abbildung. Das Ausbleiben von Spannungskontrasten und unterschiedlichem Transmissions- 
und damit Abbildungsverhalten verschiedener Kornorientierungen bezüglich des Elektronenstrahls verbessert zudem die Abbildung gegenüber den kristallinen Systemen mit Edelmetallkomponenten.

Schon in der detailreicheren Übersichtsaufnahme (Abbildung 115) werden einige typische Wachstumseffekte sichtbar, die im Weiteren diskutiert und an Beispielen dargestellt werden. Zusätzlich ist in Abbildung 115 ein typisches Feinbereichsbeugungsbild des dargestellten Schichtsystems zu sehen, das dessen amorphen Charakter zeigt. Auch für Kobalt konnten mit der Feinbereichsbeugung keine Reflexe bzw. Beugungsringe identifiziert werden Dies entspricht den Strukturuntersuchungen von Shi et al. an - bei Raumtemperatur deponierten - Kohlenstoff/KobaltSchichtsystemen, die beide Komponenten als amorph identifizierten [Shi 01].

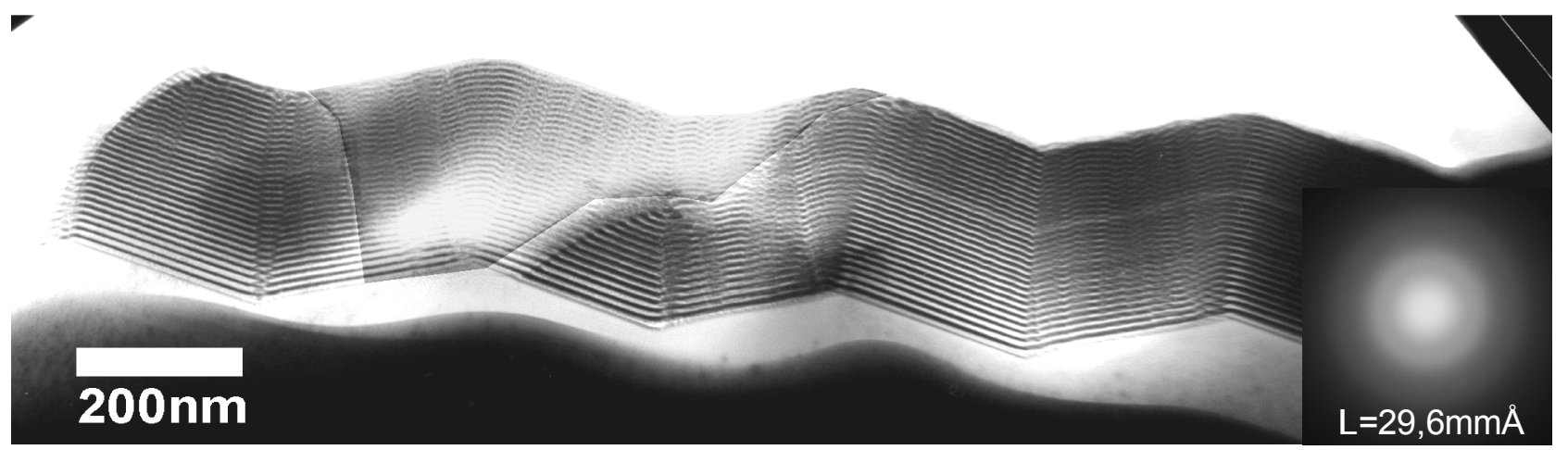

Abbildung 115: TEM-Querschnittsanalyse: 30x(2nm Kobalt/10nm Kohlenstoff) auffacettiertem $\mathrm{Al}_{2} \mathrm{O}_{3}$, Feinbereichsbeugung zeigt keine Hinweise auf kristalline Anteile.

Die Güte der gleichmäßigen, parallelen Anordnung der Einzelschichten ist wie bei den Eisen/Silberund Eisen/Gold-Schichtsystemen abhängig vom Facettenseitentyp. Während z.B. in Abbildung 115 auf den Facetten, deren Schnittlinie von links oben nach rechts unten verläuft, das Schichtpaket sehr glatt aufwächst, sind auf den Facetten mit umgekehrter Verlaufsrichtung eine Vielzahl von Störungen in der Kohärenz des Schichtsystems zu sehen. 


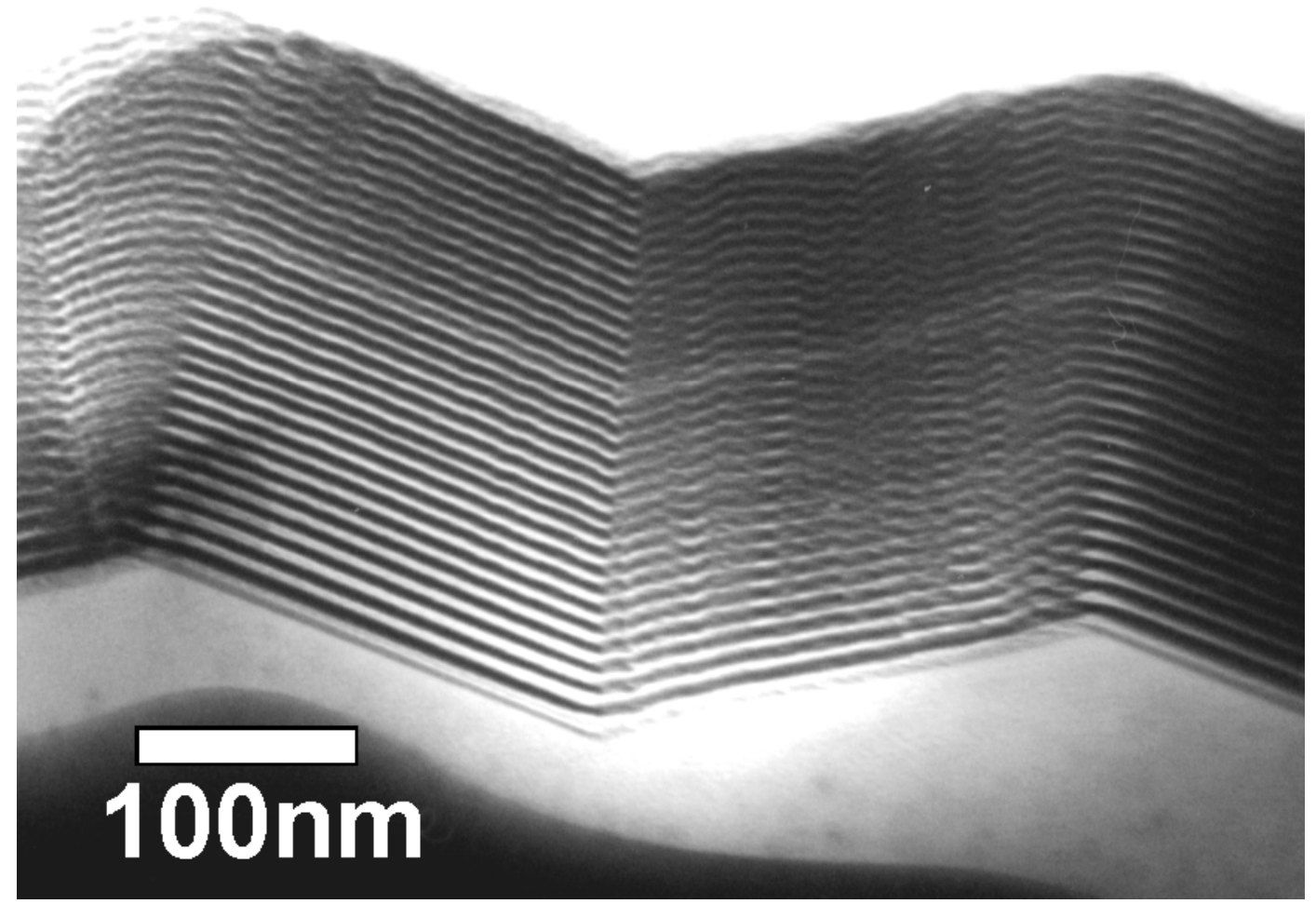

Abbildung 116: TEM-Querschnittsanalyse: 30x(2nm Kobalt/10nm Kohlenstoff) auffacettiertem $\mathrm{Al}_{2} \mathrm{O}_{3}$, Vergleich von glatter und rauer Facettenseiten.

Abbildung 116 macht deutlich, dass diese Störungen mit zunehmender Lagenzahl zunehmen. Während bei den Lagen in der Nähe des Substrat nur geringe Modulationen im geradlinigen Verlauf erkennbar sind, verstärken sich diese Fehler mit jeder Doppellage kumulativ. Dieses Wachstumsverhalten des Schichtsystems verdeutlicht die bereits im Kapitel 5.1.3 gemachte Beobachtung, dass die Facettenseiten, obwohl sie symmetrische Facettierungswinkel zeigen, eine sehr unterschiedliche Oberflächenbeschaffenheit aufweisen. Während die glatte (10 $\overline{1} 1)-\mathrm{Al}_{2} \mathrm{O}_{3}$ Kristallfläche (Abbildung 116 links) zu einer sehr regelmäßigen Schichtung im gesamten Schichtsystem und glatten Grenzflächen bis zur Oberfläche führt, sind die Nanofacetten der anderen Facettenseite (Abbildung 116 rechts) Ausgangspunkt für Störungen, die durch das darauf deponierte Schichtsystem laufen und sich kumulativ bis an die Oberfläche verstärken.

Zur Beschreibung dieser kumulativen Entwicklung führten Kardar, Parisi und Zhang (KPZ) einen nicht-linearen Term in die Wachstumsgleichung von Edwards und Wilkinson [Edw 82] ein, die eine erste lineare Beschreibung der Oberflächenentwicklung bei der Deposition granularen Materials lieferte. Dadurch wird es möglich, auch die Oberflächenevolution bei Deposition auf eine vorgegebene Oberfläche zu beschreiben. Kumulative Entwicklungen von Grenz- bzw. Oberflächen, wie im vorliegenden Fall, werden von der als KPZ-Gleichung bekannten Langevin-Gleichung wiedergegeben [Kar 86].

$$
\frac{\partial h}{\partial t}=v \nabla^{2} h+\frac{\lambda}{2}(\nabla h)^{2}+\eta(\boldsymbol{x}, t)
$$

Der zeitliche Veränderung der Höhe $h(\mathbf{x}, t)$ stehen dabei drei Terme gegenüber. Der erste entspricht einer Relaxation durch die Oberflächenspannung $v$, der zweite, nicht-lineare Term gestattet laterales 


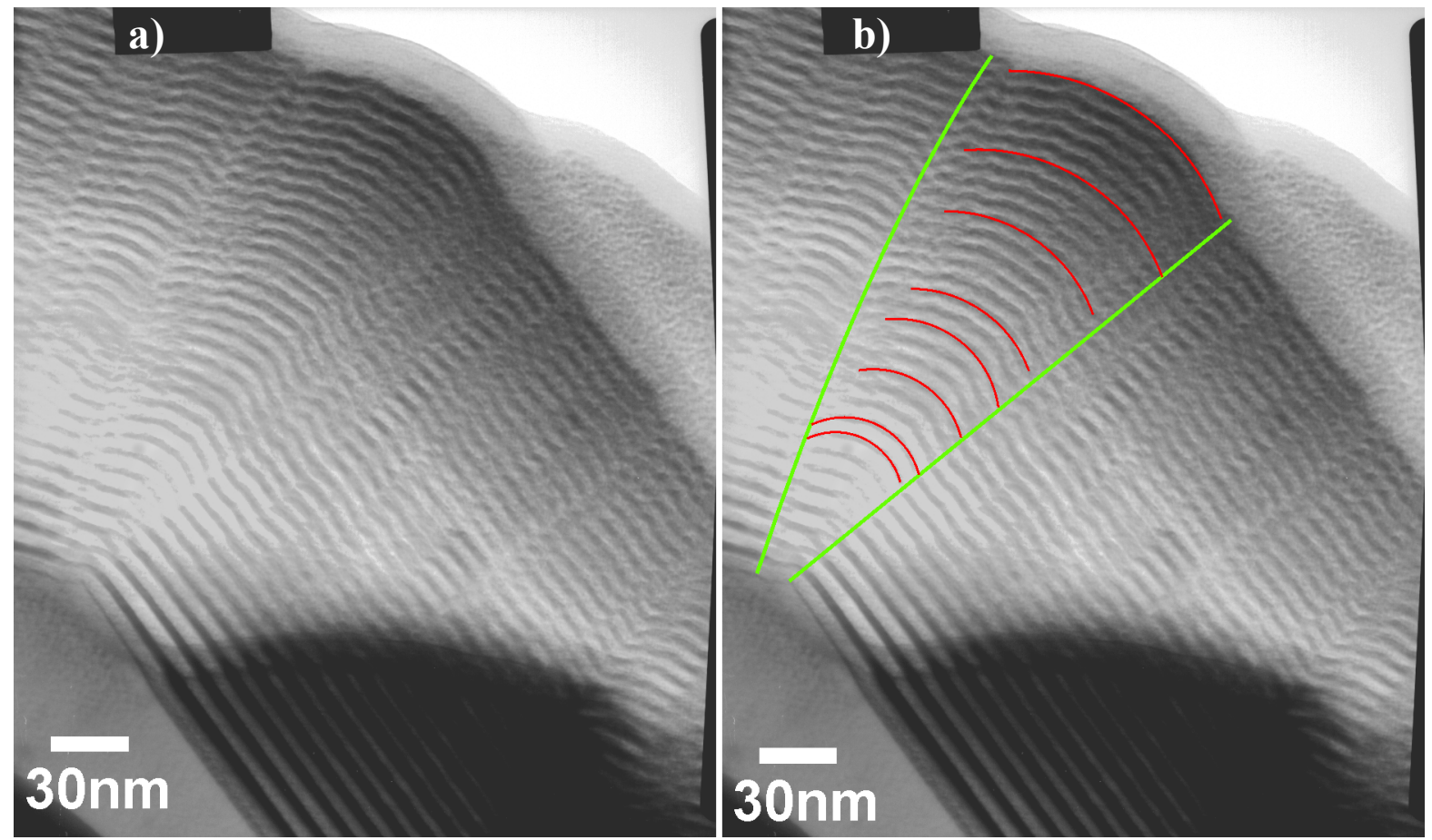

Abbildung 117: TEM-Querschnittsanalyse: Facettenspitzen führen zu starken Krümmungen der Einzelschichten. a) ohne und b) mit Hilfslinien.

Wachstum und damit auch die Bildung von Überhängen. $\eta(\mathbf{x}, t)$ repräsentiert die ortsabhängig variierende Depositionsrate.

Markante Punkte sind auch hier, wie im System Eisen/Silber, durch die Spitzen und Täler der Facettierung gegeben. Abbildung 117 zeigt, dass die Spitzen zu einer starken Krümmung der Schichten führen. Abbildung 117a zeigt das Originalbild zu sehen, in b) sind zur Verdeutlichung Hilfslinien eingefügt. Die grünen Linien zeigen die seitliche Begrenzung des Störungsbereichs an. Während die Störung an der rechten Seite durch die Oberflächennormalen der (glatten) (10 11$)$ Facettenfläche begrenzt ist, ist die Abgrenzung zur Seite der nanofacettierten Facettenfläche links leicht gebogen. Als Ursache hierfür kann die Überlagerung mit den sich nach oben verbreiternden Störungen von anderen Störstellen auf dieser Seite angesehen werden. Im Bereich zwischen den grünen Begrenzungslinien ist ab Doppellage 8 eine deutliche Krümmung der Schichten zu beobachten. Diese verläuft kreisrund, was durch die im rechten Teil rot markierten Kreissegmente verdeutlicht wird.

Eine quantitative Auswertung der Entwicklung der Krümmungsradien ergibt einen linearen Zusammenhang mit der Lagenzahl (siehe Abbildung 118). Im Bereich der ersten 5 Doppellagen ist jedoch keine signifikanten Krümmung erkennbar, vielmehr knicken hier die Einzelschichten analog zum Substratverlauf ab. Der Störungsbereich lässt sich zum Substrat hin jedoch nicht auf einen einzelnen Punkt

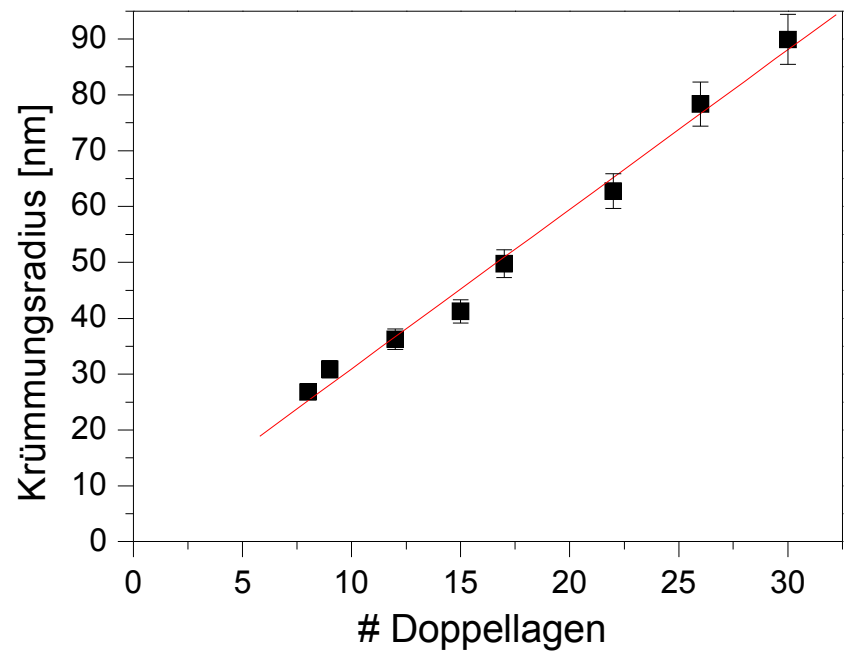

Abbildung 118: Krümmungsradius des Schichtverlaufs an einer Facettenspitze. 


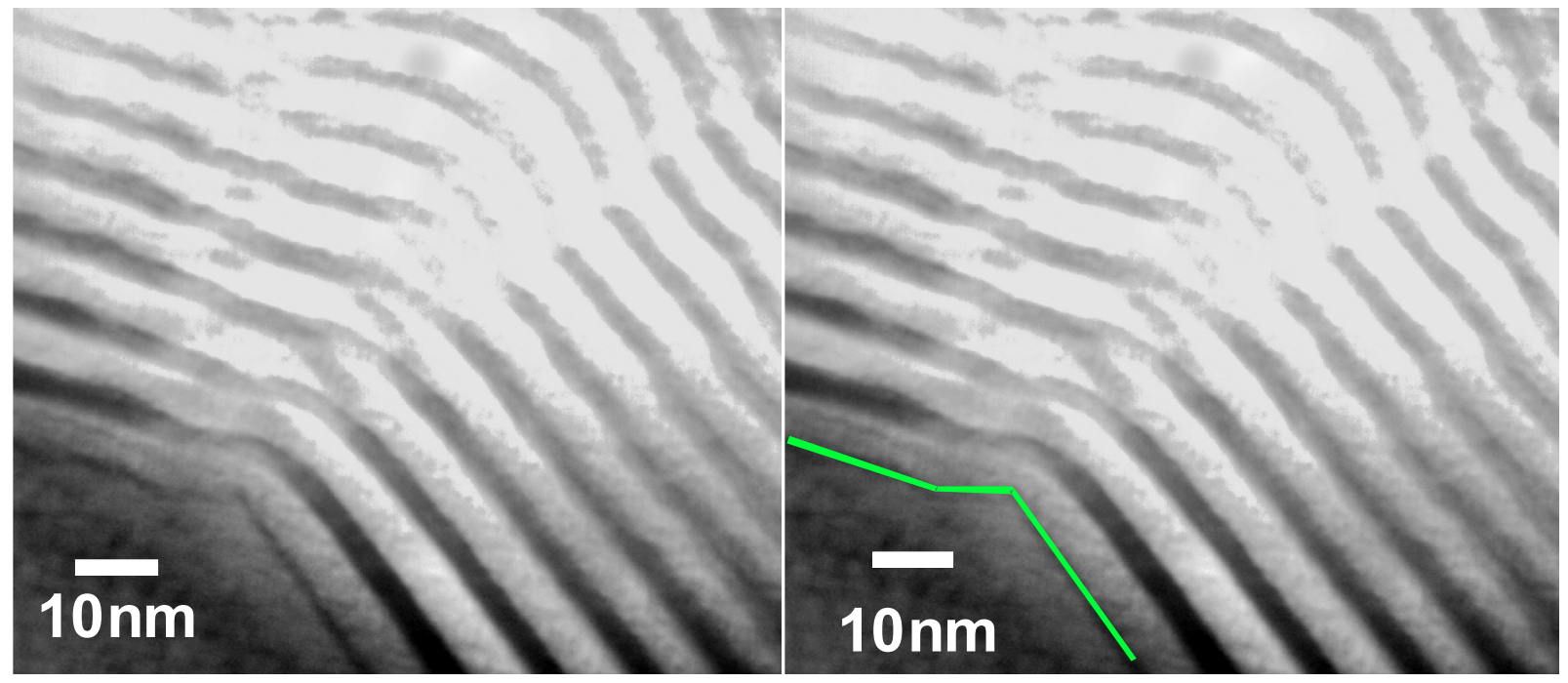

Abbildung 119: TEM-Querschnittsanalyse: (10 $\overline{1} \overline{2})$-Anstätze sind ursächlich für Störungskeile. a) ohne und b) mit Hilfslinien.

zurückführen, d.h. die beiden grünen Begrenzungslinien treffen sich nicht exakt an der Facettenspitze. Es verbleibt am Substrat ein ausgedehnter Bereich, den Abbildung 119 zeigt. Im rechten Bild ist die Substrat-Schichtsystem Grenzfläche zur Verdeutlichung nachgezeichnet. Der Ansatz der rauen Facettenseite links ist durch ein ca. $10 \mathrm{~nm}$ großes Stück $(10 \overline{1} \overline{2})-\mathrm{Al}_{2} \mathrm{O}_{3}-$ Kristallfläche gegeben. Damit entstehen zwei Knickstellen, die erste direkt an der Spitze, beim Übergang von der $(10 \overline{1} 1)$-Facettenseite auf die $(10 \overline{1} \overline{2})$-Seite und die zweite beim Übergang der $(10 \overline{1} \overline{2})$-Fläche zur mittleren Ausrichtung dieser Facettenseite. Abbildung 119 zeigt deutlich, dass diese beiden Knickstellen ursächlich für die Ausbildung des Störungkeils über das Schichtsystem hinweg sind. 


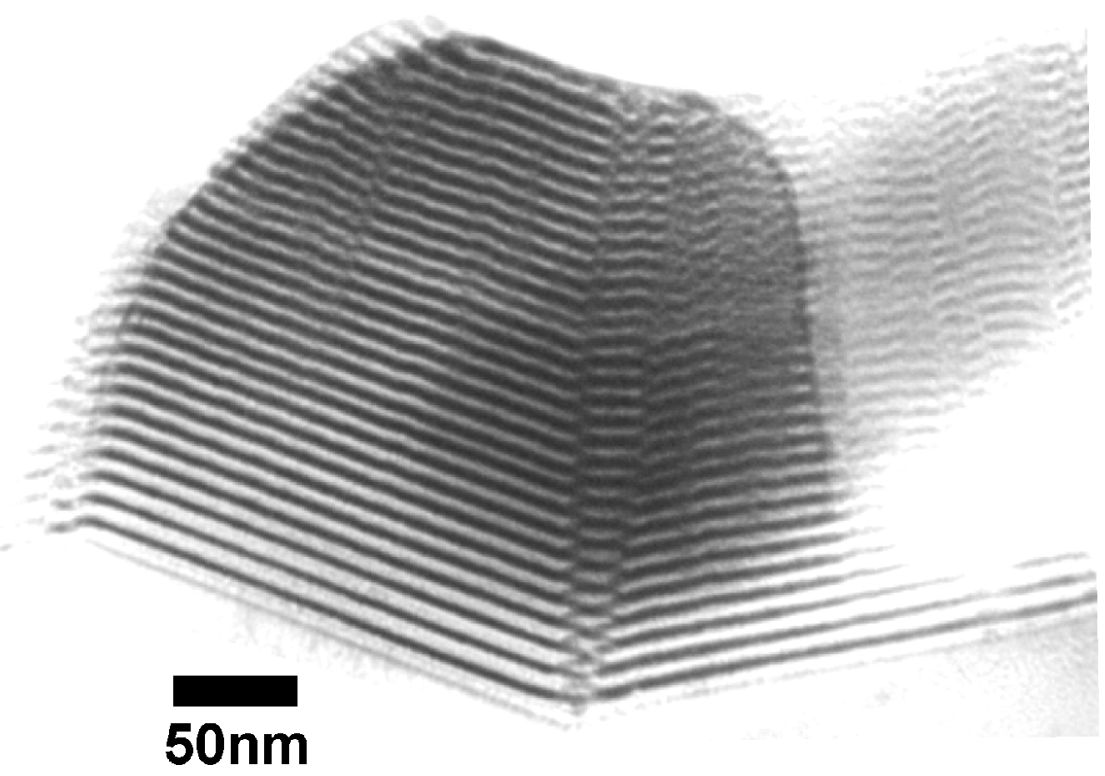

Abbildung 120: TEM-Querschnittsanalyse: Evolution der Störung oberhalb eines Facettentals.

Auch durch die Täler entstehen Störungen, die sich kumulativ durch das gesamte Schichtsystem erstrecken. Abbildung 120 zeigt exemplarisch eine solche Störung. Im Gegensatz zum Keil, der an der Spitze der Facetten entsteht, ist der Störungsbereich in diesem Fall von geringerer Ausdehnung und Verbreiterung mit steigender Schichtdicke. Die Grenzfläche im Störungsbereich verläuft gerade und ist gegenüber dem Facettenbereich erhöht. Insbesondere im mittleren Bereich des Schichtpakets entsteht dadurch ein Versatz des gestörten Bereiches um eine halbe Doppelschichtperiode gegenüber den glatten Bereichen auf den Facettenseiten. Im Gegensatz zum Eisen/SilberSchichtsystem führt die Zone oberhalb eines Facettentals hier jedoch nicht zu einer völligen Zerstörung des Schichtaufbaus.

Einzelne Oberflächenveränderungen auf einer ansonsten weitestgehend glatten Facettenseite ermöglichen es besonders gut, die Entwicklung der Störung zu studieren. Der Vergleich des Originalbilds Abbildung 121a mit Abbildung 121b, in dem ein glatter Verlauf der Co-Ebenen nachgezeichnet wurde, zeigt die sukzessive Verbreiterung der Störung mit steigender Lagenzahl. In der ersten Lage ist kaum eine Veränderung der Grenzflächen durch die Kante im Substrat zu erkennen. Nach 9-10 Doppellagen hat sich jedoch ein stark gekrümmter Verlauf der Ebenen ausgebildet, der keilförmig an Breite bis zur Oberfläche zunimmt. An der Oberseite zeigt die Überblendung mit dem geradlinigen Verlauf der Schicht, dass zusätzlich Material für die kumulative Entwicklung dieser Störung nötig ist. Der Scheitelpunkt der Krümmung liegt mehr als eine Doppelschichtdicke über dem (theoretischen) glatten Verlauf.

Diese kumulative Rauigkeitsentwicklung ist auch auf den glatten Facettenseiten zu beobachten (im oberen Drittel des Schichtpakets, siehe Abbildung 116), jedoch sind die zugrunde liegenden Welligkeiten hier deutlich flacher. Während die Ursache für die Störungen auf der anderen Facettenseite in der Beschaffenheit des Substrats zu finden ist, sind auf den glatten Facettenseiten Effekte während der Deposition des Vielfachschichtsystems entscheidend für die Ausbildung von Störungen der Übergitterstruktur. 

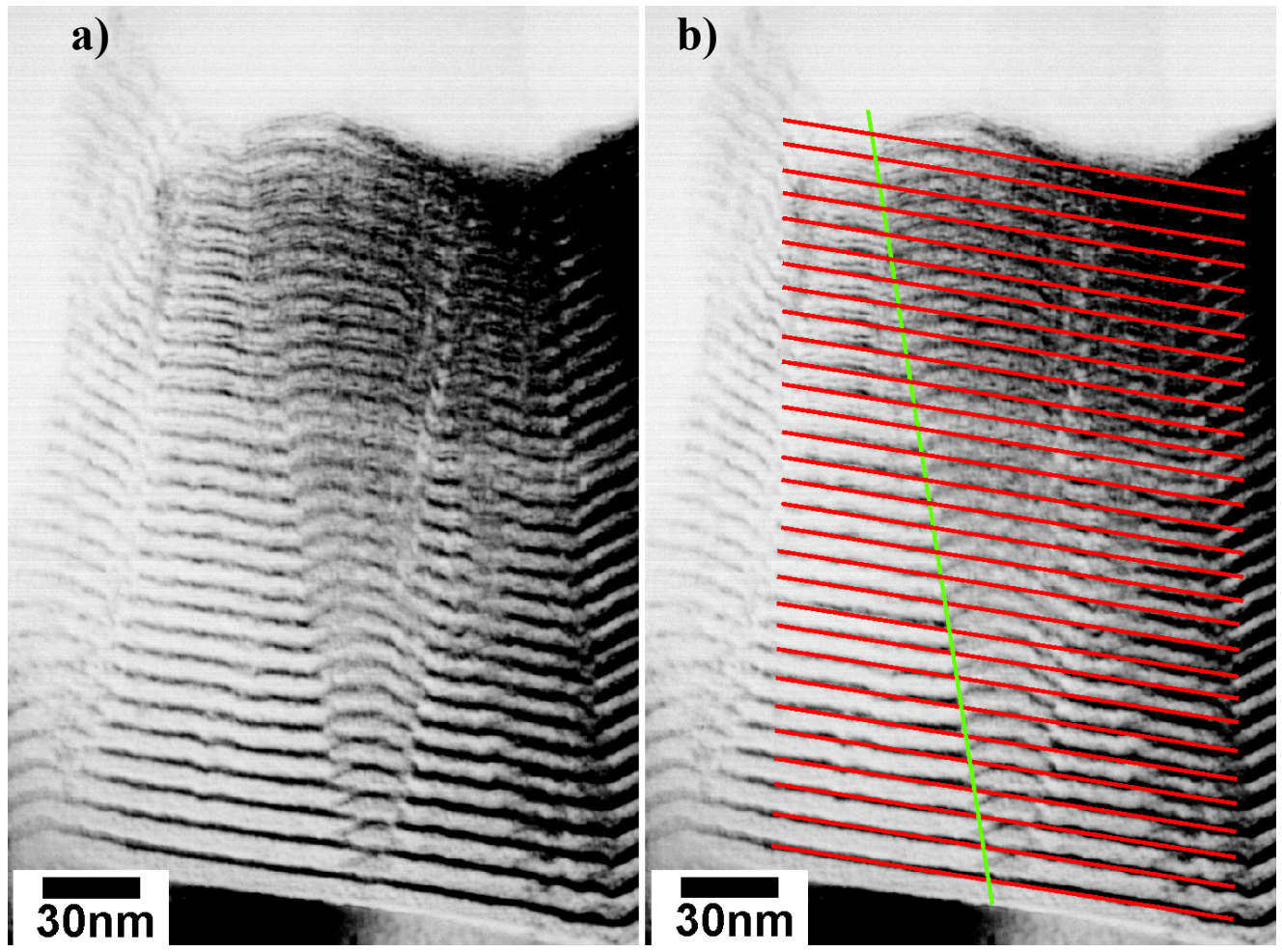

Abbildung 121: TEM-Querschnittsanalyse: Störungsentwicklung oberhalb eines einzelnen Defekts auf einer ansonsten glatten Facettenseite. a) ohne und b) mit Hilfslinien.

Damit lassen sich die Ergebnisse der Wachstumsuntersuchungen auf facettiertem $\mathrm{AbO}_{3}$ vereinfacht in Form der schematischen Abbildung 122 zusammenfassen, die sich in folgende vier markante Zonen unterteilen lässt:

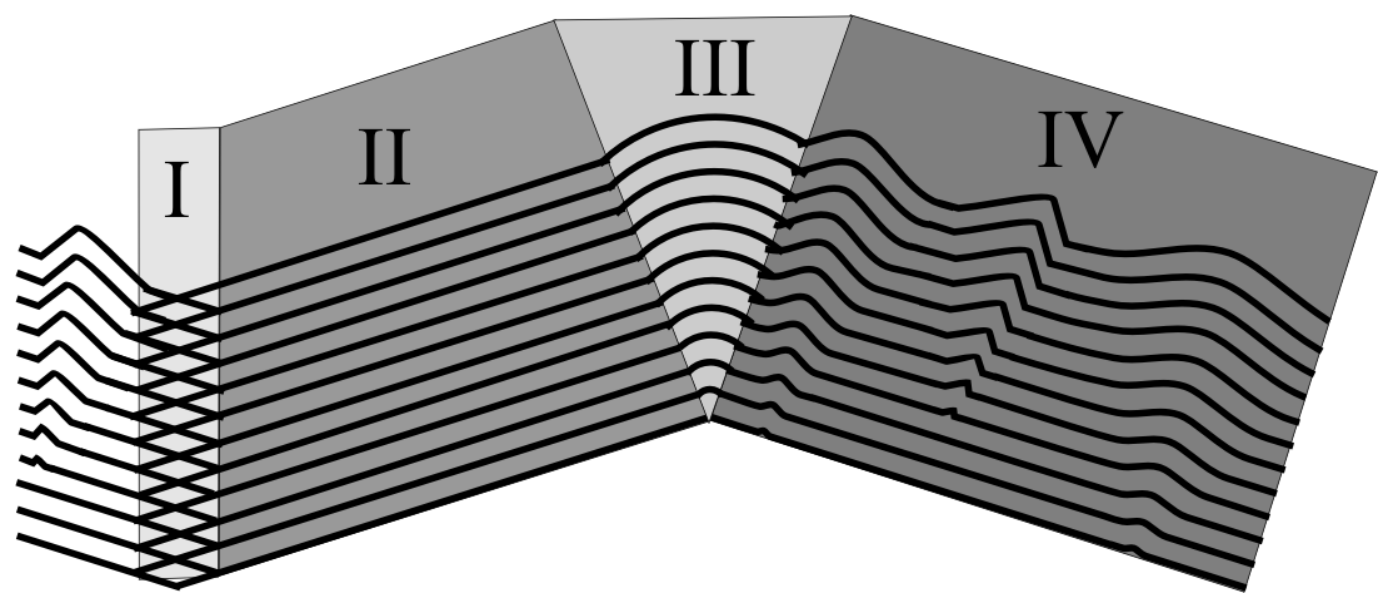

Abbildung 122: Schematische Darstellung der vier unterschiedlichen Wachstumszonen.

Zone I : $\quad$ Oberhalb der Facettentäler bildet sich eine lateral eng begrenzte Störungszone aus. Im kristallinen System Eisen/Silber ist diese Zone durch eine hohe Defektdichte und nanokristallines Gefüge gekennzeichnet. 


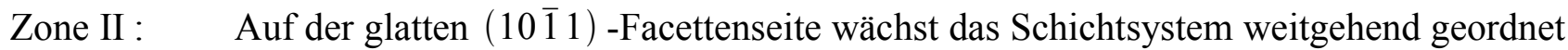
mit Grenzflächen parallel zur Facettenfläche auf. Körner wachsen in den rein metallischen Schichtsystemen mit (111)-Richtung parallel zur Facettenseitennormalen. In der Ebene liegt eine statistische Kornorientierung vor.

Zone III : Oberhalb der Facettenkämme bilden die Systeme rund gebogene Grenzflächen aus. Eisen/Silber nutzt diese Zone zur Spannungsrelaxation, wodurch in Zone II geringere Grenzflächenkrümmungen möglich werden, als sie auf glatten Substraten beobachtet werden. Das Gefüge ist in diesem Bereich, ähnlich zu Zone I, sehr defektreich.

Zone IV: Hier zeigen sich, im Kohlenstoff/Kobalt-System in beispielhafter Weise, die Aus wirkungen der nanofacettierten Facettenseite. Störungen des Substrat setzen sich kumulativ als Störungen im Aufbau des Schichtsystems fort. Im metallischen System führen diese Störungen zu einer sehr starken Beeinträchtigung des (111)-Wachstums.

Die Leitfähigkeitsmessungen konnten zeigen, dass es zu einem Inselwachstum bei der Depsition der ersten Eisenschicht auf den Facetten kommt, die TEM-Querschnittsuntersuchungen bestätigen dies. Eine Trennung von Eisen- und Silberlagen ist erst nach der ersten Silberlage möglich, durch die die Bereiche zwischen den Eiseninseln aufgefüllt werden. Die Störungszonen I und III haben nur geringen Einfluss auf die Isotropie der Leitfähigkeit in den Messrichtungen parallel und senkrecht zur den Facetten. Die Texturmessungen zeigen eine (111)-Drahttextur der Edelmetalle auf den Facettenseiten, die TEM-Untersuchungen an Eisen/Silber bestätigen das Wachstum entsprechender Körner parallel zu den Facettenseitennormalen über mindestens zehn Doppellagen hinweg. 


\subsection{Gestaltumwandlung durch Temperaturbehandlung}

Die Temperung von Nanodrähten auf der facettierten Saphiroberfläche hat gezeigt, dass es oberhalb einer kritischen Temperatur zu einer Gestaltänderung kommt und die Drähte in Reihen von separierten Partikeln überführt werden können. Die intensive Charakterisierung der Schichtsysteme auf facettiertem $\mathrm{Al}_{2} \mathrm{O}_{3}$ führte $\mathrm{zu}$ dem Ergebnis, dass die Facetten die Mikrostruktur des Schichtsystems lokal durch Defektzonen stark verändern. Fraglich ist, ob diese Veränderungen einen messbaren Einfluss auf die bekannte thermisch induzierte Gestaltänderung haben, die eine Anordnung sphärischer Präzipitate in eben diesen defektreichen Zonen erwarten lässt [Her 99], [Tro 00].

\subsubsection{Thermische Charakteristik der Gestaltumwandlung}

Die Gestaltänderung von Einzelschichten zu separierten Partikeln findet in Vielfachschichtsystemen in einen endlichen Temperaturbereich statt, der durch verschiedene Analyseverfahren einzugrenzen war. Daher wurde die Einsatztemperatur, bei der erste Anzeichen einer Unterbrechung des geschichteten Aufbaus beobachtet werden können, mittels in-situ Leitfähigkeitsmessungen während der Temperung und in-situ Röntgendiffraktometrie bestimmt. Außerdem ergeben sich Hinweise auf Temperaturen, bei denen der Zerfall der Schichtsysteme abgeschlossen ist.

Ein neu konzipierter und konstruierter Halter zur in-situ Leitfähigkeitsmessung ermöglichte durch die Kombination mit einem leistungsstarken Bornidrid-Heizer die Temperung der Schichtsysteme direkt im Anschluss an die Deposition, wodurch Verunreinigungen, Oxidation und weitere störende Einflüsse durch den Kontakt der Proben mit der Atmosphäre vermieden wurden.

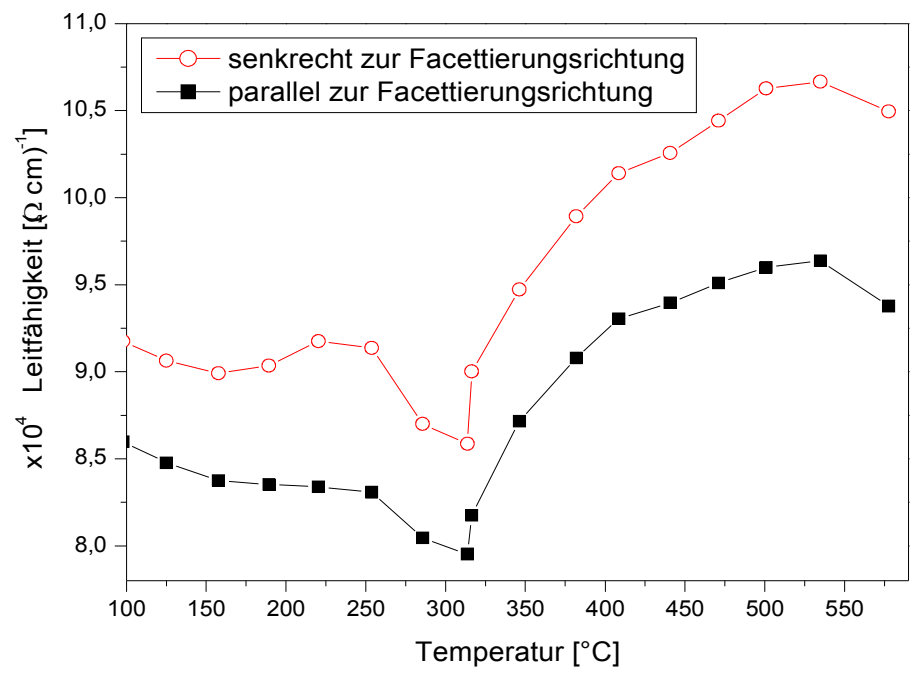

Abbildung 123: Leitfähigkeitsveränderung während Temperung bis $580^{\circ} \mathrm{C}$ eines $50 x(2 \mathrm{~nm} \mathrm{Fe} / 10 \mathrm{~nm} \mathrm{Ag})$-Schichtpakets auf facettiertem $\mathrm{Al}_{2} \mathrm{O}_{3}$.
Wie schon während des Wachstums der Schichtsysteme gezeigt, ist eine simultane Messung der Leitfähigkeit in den zwei Richtungen parallel zur Facettierungsrichtung und senkrecht dazu möglich.

Abbildung 123 zeigt exemplarisch die Leitfähigkeitsveränderung während der Temperung eines Eisen/Silber-Schichtsystems auf facettiertem Substrat bis zur Endtemperatur von $580^{\circ} \mathrm{C}$.

Bis $\mathrm{zu}$ einer Temperatur von $314^{\circ} \mathrm{C}$ nimmt die Leitfähigkeit des Schichtpakets in beiden Messrichtungen ab. Dies ist durch eine erhöhte Streuung an Phononen zu erklären. Bei $314^{\circ} \mathrm{C}$ steigt die Leitfähigkeit in beiden Messrichtungen sprunghaft an. Diese

Temperatur kann als Einsatzpunkt für die thermische Zerstörung der Eisenschichten in separierte Partikel festgelegt werden. Durch den direkten Kontakt benachbarter Silberschichten beim Durchbrechen der schlecht leitfähigen Eisenschichten verbessert sich die gesamte Leitfähigkeit des Systems sprunghaft. Im weiteren Verlauf der Temperung konkurriert zunächst die 
Leitfähigkeitssteigerung durch weitere Kurzschlüsse durch die Eisenschichten hindurch bis zur vollständigen Umformung der Eisenschichten in separierte Partikel mit der Leitfähigkeitsminderung durch Phononenstreuung. Die maximale Leitfähigkeit wird in beiden Messrichtungen bei $\sim 530^{\circ} \mathrm{C}$ mit $9,64 \cdot 10^{4}(\Omega \mathrm{cm})^{-1}$ parallel zur Facettierungsrichtung bzw. $10,67 \cdot 10^{4}(\Omega \mathrm{cm})^{-1}$ senkrecht dazu erreicht, womit wahrscheinlich der Abschluss der Gestaltumwandlung bei diesem Temperungsprofil angezeigt wird.

Die Messungen am Schichtsystem Eisen/Silber zeigen also, dass sich die Veränderungen in der Leitfähigkeit über einen vergleichsweise großen Temperaturbereich von mindestens $70^{\circ} \mathrm{C}$ erstrecken. Die Gestaltänderung ist damit kein spontaner Prozess. Zwar ist der erste Durchbruch der Eisenschichten durch einen scharf definierten Anstieg der Leitfähigkeit gekennzeichnet, aber der Ablauf der weiteren Veränderungen läuft als diffusionsgesteuerter Prozess in einem TemperaturZeit-Fenster ab.

Interessant ist ferner, dass der charakteristische Anstieg auf glattem Substrat bei $350^{\circ} \mathrm{C}$ beginnt [Her 02], während der Einsatzpunkt durch die Facettierung des Substrat auf $314^{\circ} \mathrm{C}$ sinkt.

Im System Eisen/Gold werden vergleichbare, charakteristische Veränderungen der Leitfähigkeit bei der Zerfallstemperatur beobachtet. Es schließt sich ebenso ein Temperaturfenster von $\sim 310^{\circ} \mathrm{C}$ bis $390^{\circ} \mathrm{C}$ an, das der Gestaltänderung der Eisenschicht zugeschrieben werden kann. Die höhere Löslichkeit von Eisen in der Goldmatrix (gegenüber einer Silbermatrix) führt in diesem Fall aber zu einer Unterdrückung des Leitwertanstiegs.

Kohlenstoff/Kobalt-Schichtsysteme sind hingegen insbesondere auf glattem Substrat bis zu sehr hohen Temperaturen $\left(>600^{\circ} \mathrm{C}\right)$ thermisch stabil. Auf facettiertem Substrat deutet jedoch ein Abfall der Leitfähigkeit bereits oberhalb von $\sim 430^{\circ} \mathrm{C}$ in Messrichtung senkrecht zur Facettierung auf anisotrope Veränderungen im Schichtsystem hin (Abbildung 124). Durch die Wahl einer facettierten m-plane $\mathrm{Al}_{2} \mathrm{O}_{3}$-Oberfläche als Substrat und einer gezielten Temperung im Temperaturbereich zwischen $430^{\circ} \mathrm{C}$ und $600^{\circ} \mathrm{C}$, kann die morphologische Anisotropie des Substrats in eine Anisotropie im Transportverhalten überführt werden. Als Ursache hierfür wird eine Unterbrechung der stromtragenden Kobaltschicht im Bereich der Facettenspitzen bzw. -täler als sehr

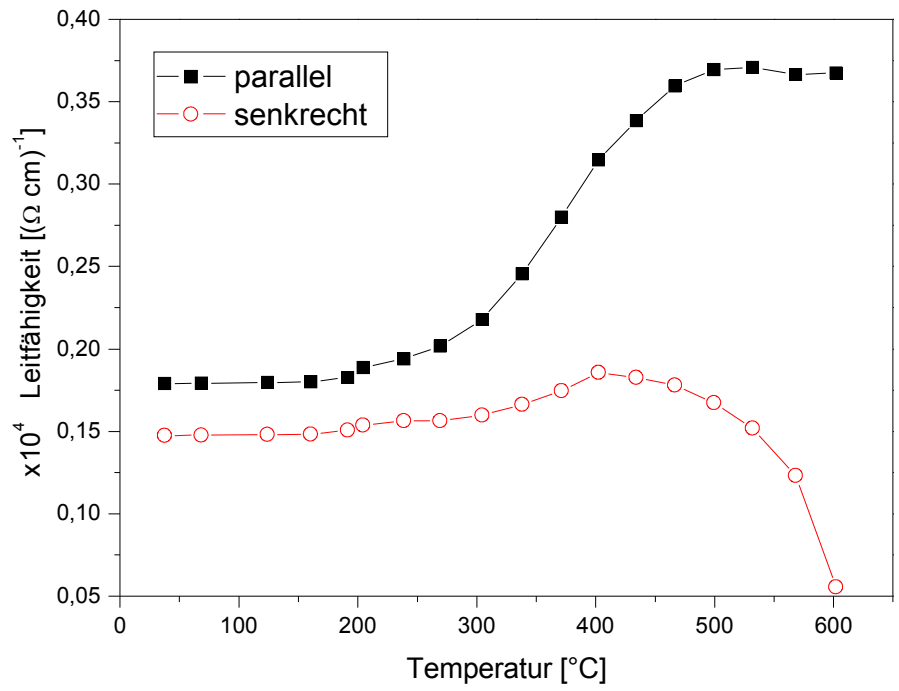

Abbildung 124: Leitfähigkeitsänderung durch die Temperung eines $20 x(2 \mathrm{nmCo} / 10 \mathrm{~nm} \mathrm{C})$ Schichtsystems auf facettiertem $\mathrm{Al}_{2} \mathrm{O}_{3}$ bis $600^{\circ} \mathrm{C}$. wahrscheinlich angesehen, da die Analysen zum Wachstum der Schichtsysteme diese Stellen als Bereiche stärkster mikrostruktureller Störung im Schichtaufbau identifizieren konnten.

Im Folgenden soll die thermische Zersetzung der Schichtpakete anhand von in-situ röntgendiffraktometrischen Messungen untersucht werden. 
In Abbildung 125 sind die Diffraktogramme eines Eisen/GoldSchichtsystems auf facettiertem m-plane $\mathrm{Al}_{2} \mathrm{O}_{3}$ vor und nach einer Temperbehandlung bis $400^{\circ} \mathrm{C}$ gezeigt. Vor der Temperung ist in der Umgebung der (111)-Orientierung die bereits erläuterte typische, periodisch modulierte Intensitätsverteilung zu erkennen. Neben dem stärksten Reflex bei ca. $45^{\circ}$ sind mehrere Satelliten bei ca. $42^{\circ}, 43^{\circ}, 44^{\circ}$ sowie $46^{\circ}$ und $47^{\circ}$ sichtbar. Nach der Temperung ist die Übergitterperiodizität zerstört, mit der Konsequenz, dass die Überstrukturreflexe entfallen und die Intensitätsverteilung sich alleine aus der Beugung an Kristallebenen ergibt. Entsprechend verbleibt nach der Temperung im hier dargestellten

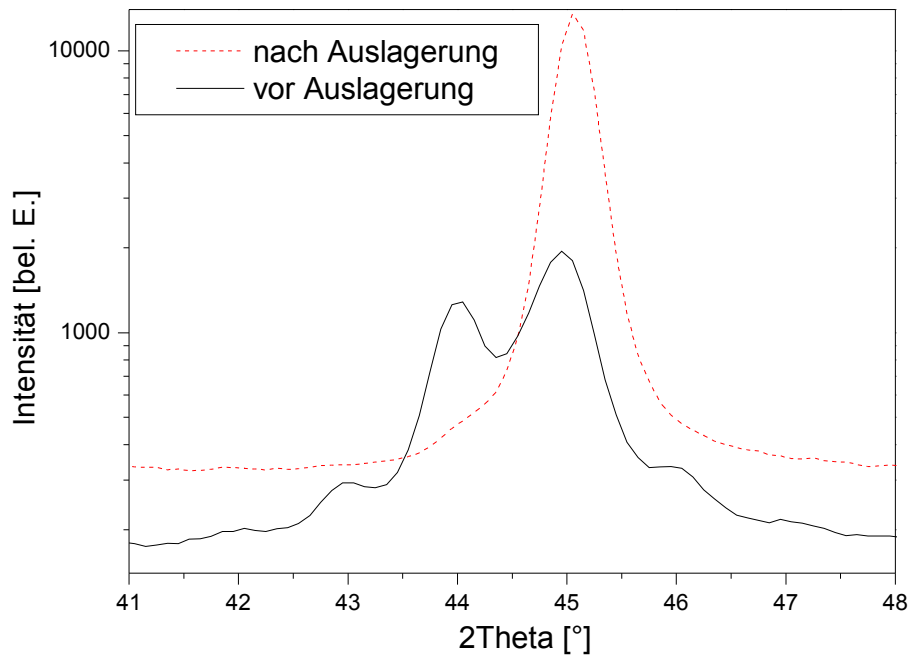

Abbildung 125: XRD: Vergleich von Diffraktogrammen vor und nach der Temperung bis $400^{\circ} \mathrm{C}$. Schichtsystem:

$50 x(2 \mathrm{~nm} \mathrm{Fe} / 10 \mathrm{~nm} \mathrm{Au})$ auffacettiertem m-plane $\mathrm{Al}_{2} \mathrm{O}_{3}$; horizontal beschichtet. Winkelbereich nur der $\mathrm{Au}(111)-$ Kristallreflex.

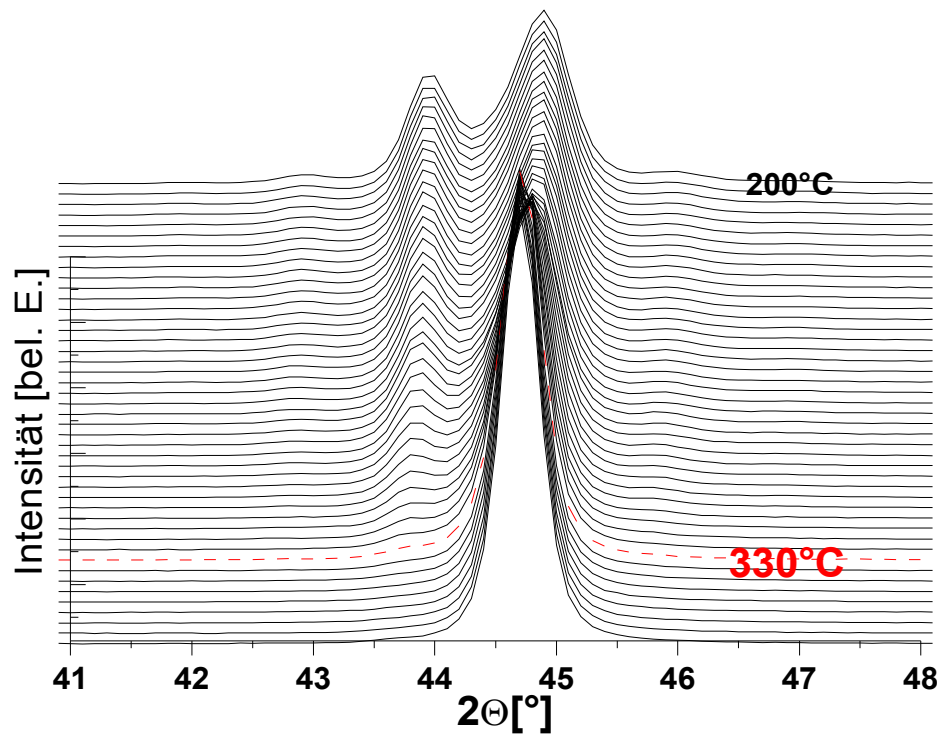

Abbildung 126: Veränderung des Röntgenspektrums während der Temperung von $200^{\circ} \mathrm{C}$ bis $400^{\circ} \mathrm{C}$ eines $50 x(2 \mathrm{~nm} \mathrm{Fe} / 10 \mathrm{~nm} \mathrm{Au})$ Schichtsystems auffacettiertem m-plane $\mathrm{Al}_{2} \mathrm{O}_{3}$; horizontal beschichtet.
Abbildung 126 zeigt die ganze Abfolge von Diffraktogrammen im Winkelbereich von $41^{\circ}-48^{\circ}$ während des Temperprozesses im Hochvakuum von $200^{\circ} \mathrm{C}$ bis $400^{\circ} \mathrm{C}$ bei einer Heizrate von $0,2 \mathrm{~K} / \mathrm{min}$, von oben nach unten mit zunehmender Temperatur. (Die Satellitenreflexe geringerer Intensität bei niedriger Temperatur sind in der linearen Intensitätsskalierung weniger deutlich erkennbar). Gut sichtbar ist, dass die Höhe des Satellitenreflex bei 43,7 ${ }^{\circ}$ mit steigender Temperatur sinkt. Gleichzeitig wächst der Hauptreflex mit simultan sinkender Halbwertsbreite. 


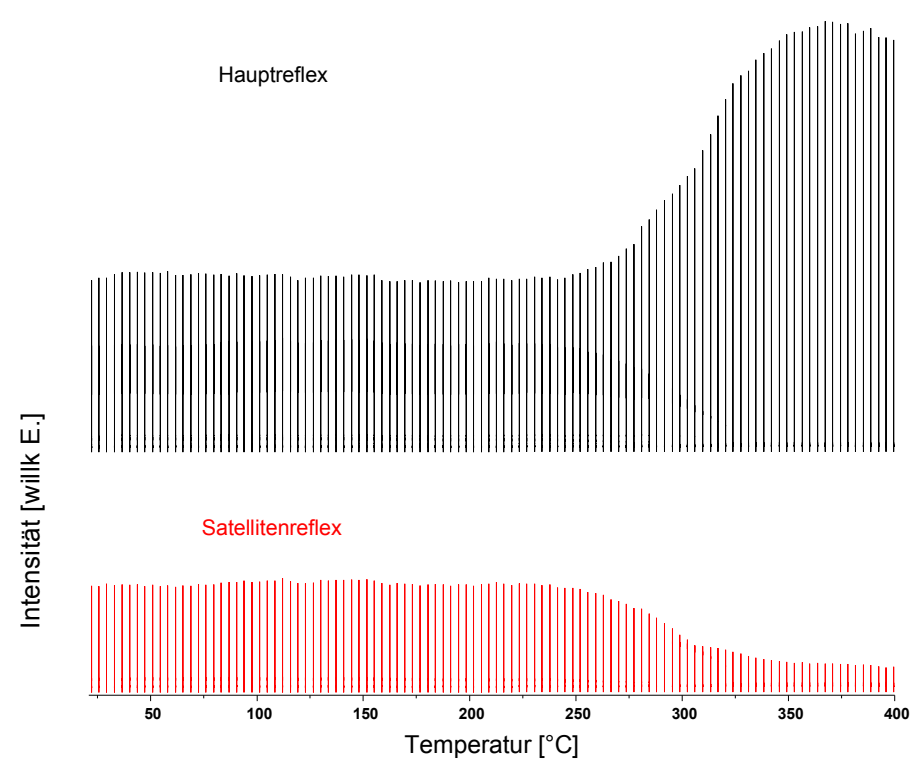

Abbildung 127: Intensitätsentwicklung des Au-(111)-Hauptreflexes und des Satellitenreflexes bei $2 \Theta=43,7^{\circ}$ während der Temperung eines $50 x(2 \mathrm{~nm} \mathrm{Fe} / 10 \mathrm{~nm} \mathrm{Au})$-Schichtsystems auf facettiertem m-plane $\mathrm{Al}_{2} \mathrm{O}_{3}$ bis $400^{\circ} \mathrm{C}$; horizontal beschichtet.
Trägt man die maximale Intensität des Haupreflexes bzw. des Satellitenreflexes bei $43,7^{\circ}$ gegen die Temperatur auf (Abbildung 127), so ist der Temperaturbereich, in dem die wesentlichen mikrostrukturellen Veränderungen im Schichtsystem stattfinden, besser $\mathrm{zu}$ erkennen. Es ist auffällig, dass die Intensitätszunahme des Hauptreflexes und die Intensitätsabnahme des Satellitenreflexes simultan erfolgen, d.h. der Verlust der Übergitterperiodizität geht mit der Steigerung der Röntgenkohärenztiefe einher. Eine Definition der Kohärenztiefe ist im Kapitel 2.3 gegeben. Abweichungen in den Intensitätsverläufen werden $\mathrm{ab} 270^{\circ} \mathrm{C}$ sichtbar, gefolgt von einem Bereich großer Steigung zwischen $280^{\circ} \mathrm{C}-330^{\circ} \mathrm{C}$ und einem Bereich weitgehend konstanter Intensität $\mathrm{ab} 350^{\circ} \mathrm{C}$.

Abbildung 128 zeigt zum Vergleich eine solche Intensitätsentwicklung einer in-situ Röntgenanalyse eines Eisen/Silber-Schichtsystems während einer Temperung bis $500^{\circ} \mathrm{C}$, ebenfalls bei einer Heizrate von $0,2 \mathrm{~K} / \mathrm{min}$.

Der Verlauf ist ähnlich, jedoch zeigt sich, dass die Analyse schwieriger ist, da in diesem Fall der stärkste Satellitenreflex bei der Temperung im Hauptreflex aufgeht. Es existiert kein scharf definierter Übergang für die Steigerung der Intensität des Hauptreflexes. Für den Satellitenreflex beginnt der Intensitätsrückgang bei $\sim 240^{\circ} \mathrm{C}$, vollständig verschwunden sind die Satellitenreflexe in diesem System

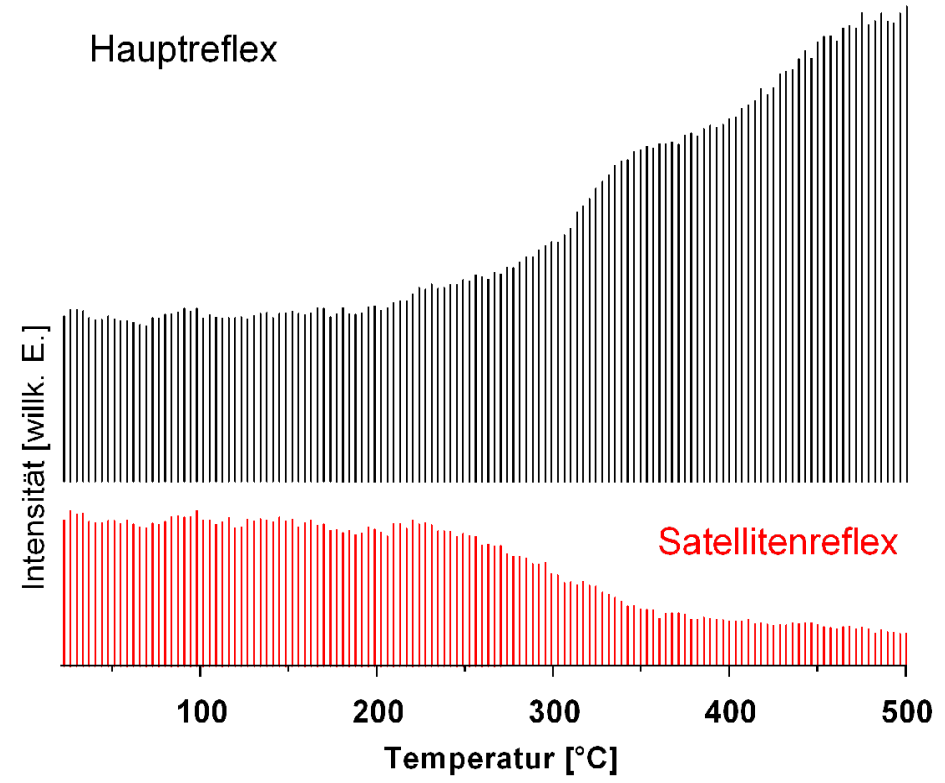

Abbildung 128: Intensitätsentwicklung des Ag-(111)-Hauptreflexes und des stärksten Satellitenreflexes während der Temperung bis $500^{\circ} \mathrm{C}$ eines $20 x(2 \mathrm{nmFe} / 10 \mathrm{~nm} \mathrm{Ag})$-Schichtsystems auf facettiertem m-plane $\mathrm{Al}_{2} \mathrm{O}_{3}$; horizontal beschichtet. bei einer Temperatur von $350^{\circ} \mathrm{C}$.

$\mathrm{Da}$ das System Eisen/Silber trotz sonst gleicher Parameter wie z.B. Einzellagendicke und Gesamtlagenzahl wesentlich schwächere Überstrukturen in der Großwinkelröntgenbeugung zeigt als Eisen/Gold und durch die facettierte Oberfläche zusätzlich massive Intensitätseinbußen hinzunehmen sind, wurden detailliertere in-situ röntgendiffraktometrische Messungen mit einer für diese Zwecke neu konzipierten Vakuum-Messkammer am Hamburger Synchrotron DESY/HASYLAB an der Beamline B2 durchgeführt. Ein großer Vorteil ist die hohe Brillianz der Quelle, weshalb auch Untersuchungen an solchen Proben niedriger Streuintensität möglich sind. 

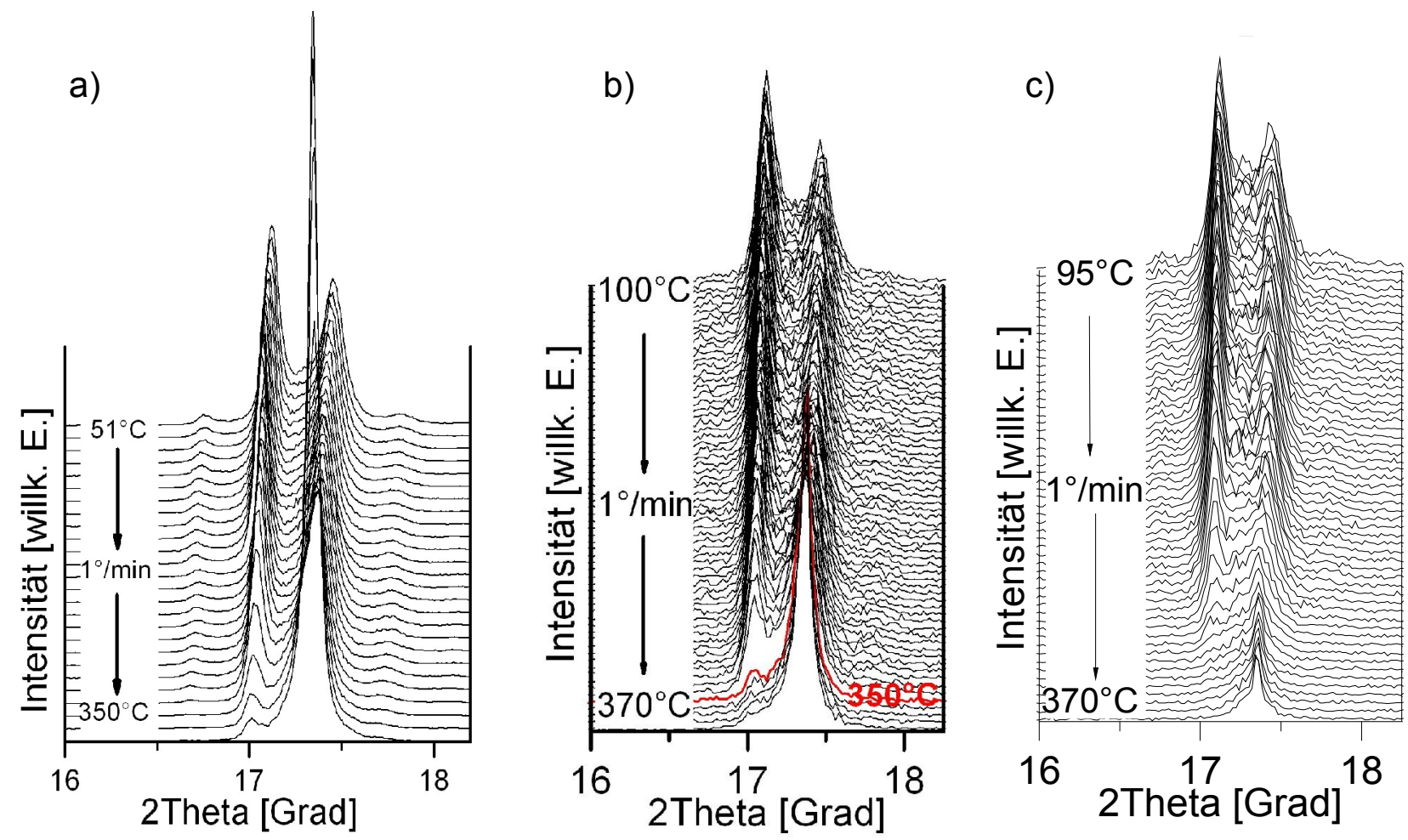

Abbildung 129: XRD-Temperung eines 50x(2nm Fe/10nm Au) Schichtsystems auf: a) glattem $\mathrm{Al}_{2} \mathrm{O}_{3}$ bis $350^{\circ} \mathrm{C}$,

b) facettiertem $\mathrm{Al}_{2} \mathrm{O}_{3}$, horizontale Beschichtung bis $370^{\circ} \mathrm{C}$, c) facettiertem $\mathrm{Al}_{2} \mathrm{O}_{3}$, vertikale Beschichtung bis $370^{\circ} \mathrm{C}$.

Alle Proben einer Materialkombination für dies Untersuchungen wurden im selben Prozess hergestellt, um die Vergleichbarkeit sicherzustellen, denn die Einzellagendicke hat wesentlichen Einfluss auf die Umwandlungstemperatur [Tro 00].

Abbildung 129 zeigt anhand von Abfolgen der Diffraktogramme vergleichend die Veränderung des Schichtaufbaus während der gesamten Temperungsphasen (mit einer Heizrate von 1K/min auf $350^{\circ} \mathrm{C}$ bzw. $370^{\circ} \mathrm{C}$ ) gleicher Eisen/Gold-Schichtsysteme auf glattem (a) und auf facettiertem $\mathrm{Ab}_{3}$ (b und c). Die Beschichtungsgeometrie mit homogener Materialverteilung bei horizontaler Ausrichtung der Facettierung (b) und präferenzieller Materialverteilung bei vertikaler Ausrichtung der Facettierung (c) führt dabei zu unterschiedlichen Endzuständen. 
a)

\section{Hauptreflex}

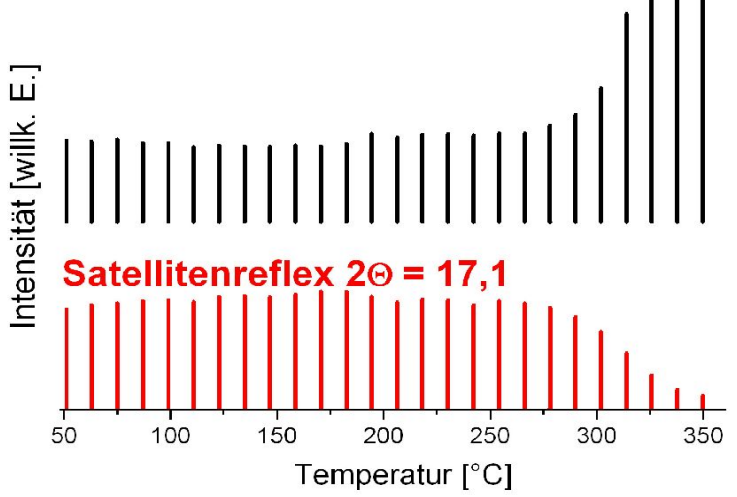

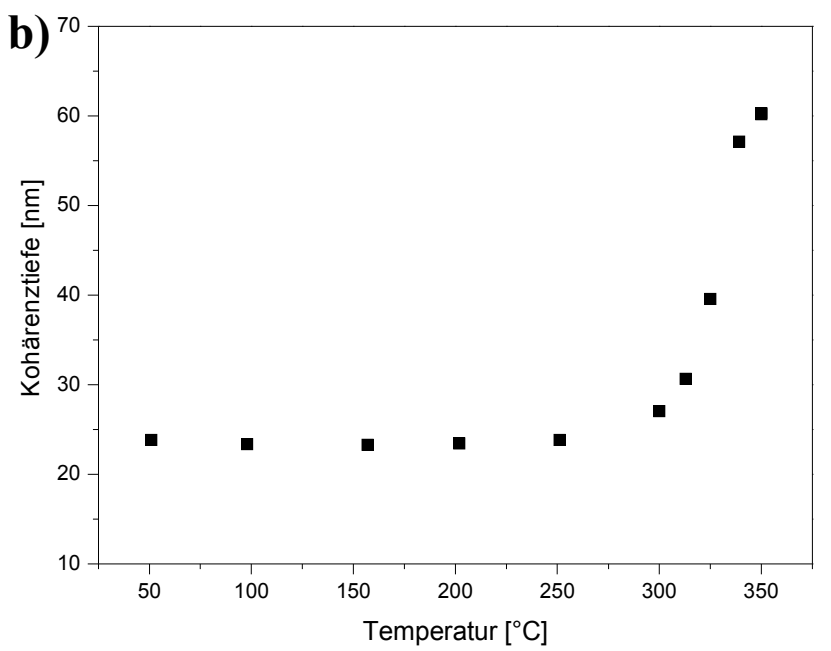

Abbildung 130: a) Intensitätsentwicklung des Au(111)-Hauptreflexes und des Satellitenreflexes und b) Kohärenztiefe während der Temperung eines $50 x\left(2 \mathrm{~nm} \mathrm{Fe} / 10 \mathrm{~nm}\right.$ Au)-Schichtsystems auf glattem $\mathrm{Al}_{2} \mathrm{O}_{3}$ bis $350^{\circ} \mathrm{C}(\mathrm{zu}$ Abbildung $129 a)$.

Abbildung 130a zeigt den temperaturabhängigen Intensitätsverlauf des zunächst intensiveren Satellitenreflexes bei ca. $17,1^{\circ}$ und des zweiten Hauptreflexes bei ca. 17,4 ${ }^{\circ}$, der nach Zerfall des Schichtaufbaus in den $\mathrm{Au}(111)$-Kristallreflex übergeht, für die Probe auf glattem $\mathrm{Al}_{2} \mathrm{O}_{3}$. Eine Veränderung des Schichtaufbaus findet demnach im Bereich zwischen etwa $275^{\circ} \mathrm{C}$ und $350^{\circ} \mathrm{C}$ statt Abbildung 130b zeigt die temperaturabhängige Entwicklung der Kohärenztiefe, bestimmt mit der Scherrer-Formel aus der Reflexbreite und -position bei ca. 17,4 ${ }^{\circ}$. Der Vergleich der Messungen in Abbildung 130 verdeutlicht, dass einhergehend mit dem Verschwinden der Überstruktur und der starken Zunahme der Intensität im Hauptreflex auch die Kohärenztiefe ansteigt. Beginnend $\sim 270^{\circ} \mathrm{C}$ erstreckt sich der Bereich stärkster Veränderungen dieser Größen auf Temperaturen zwischen $320^{\circ} \mathrm{C}$ und $340^{\circ} \mathrm{C}$. Damit zeigt der Vergleich mit Ergebnissen auf (0001)- $\mathrm{Al}_{2} \mathrm{O}_{3}$ und Silizium mit Diffusionsbarrieren [Her 99], [Tro 00], dass die Zerfallstemperatur weitgehend unabhängig von der exakten Kristallorientierung des Substrat ist. Da in der Regel bei der Deposition bei Raumtemperatur keine Epitaxie zum Substrat auftritt, ist auch der Einfluss

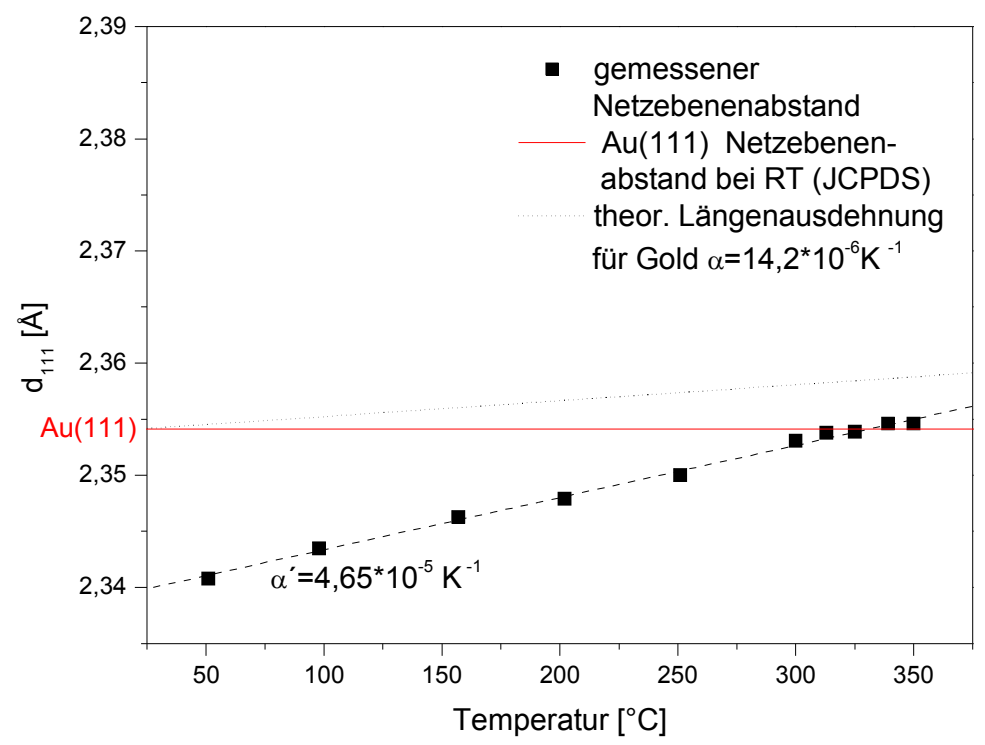

Abbildung 131: Ausdehnung der (111)-Netzebenenabstände durch die Temperung eines $50 x(2 \mathrm{~nm} \mathrm{Fe} / 10 \mathrm{~nm} \mathrm{Au})$-Schichtsystems auf glattem $\mathrm{Al}_{2} \mathrm{O}_{3}$ bis $350^{\circ} \mathrm{C}$ im Vergleich zur theoretischen Ausdehnung. der atomaren Anordnung unter dem Schichtsystem für dessen Wachstum gering.

Anhand der genauen Lage des (111)-Reflexes wurden ferner die Spannungszustände der deponierten Schichtsysteme untersucht. Durch die Temperung erwartet man aufgrund der thermischen Ausdehnung eine Vergrößerung des Netzebenenabstands. Im relevanten Temperaturbereich von Raumtemperatur bis $350^{\circ} \mathrm{C}$ ist die Ausdehnung von Metallen und im Speziellen von Gold weitgehend linear und isotrop mit einem Längenausdehnungskoeffizienten

$\alpha_{A u}=14,2 \cdot 10^{-6} \mathrm{~K}^{-1}$ [Dax 03]. Abbildung 131 zeigt jedoch für das 


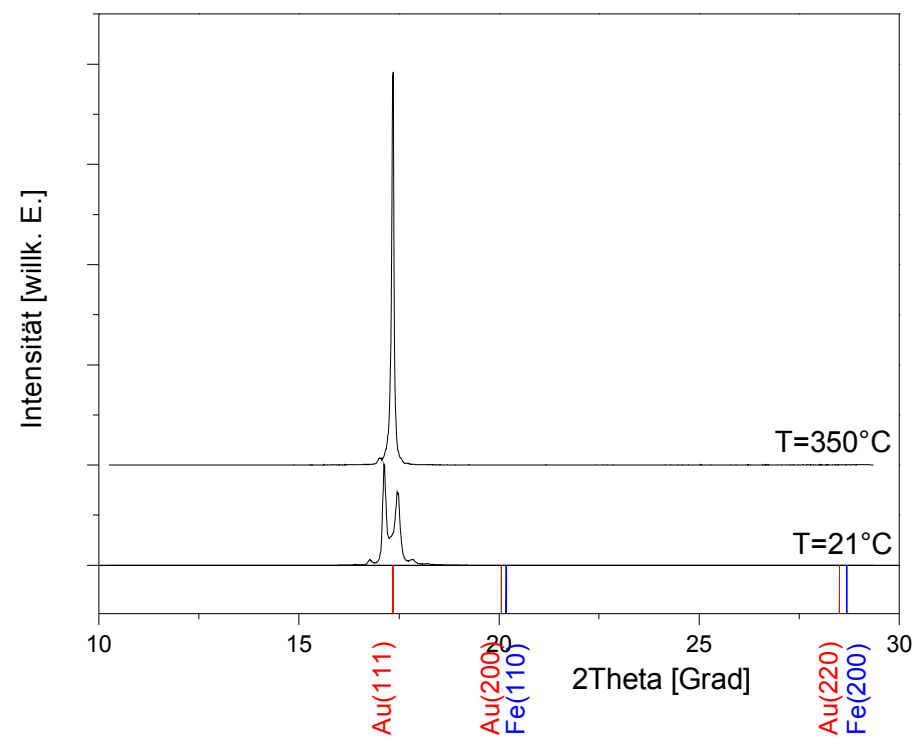

Abbildung 132: XRD-Diffraktogramme vor und nach der Temperung eines $50 x(2 \mathrm{~nm} \mathrm{Fe} / 10 \mathrm{~nm} \mathrm{Au})$ Schichtsystems auf glattem $\mathrm{Al}_{2} \mathrm{O}_{3}$ bis $350^{\circ} \mathrm{C}$.

Eisen/Gold-Schichtsystem auf glattem Substrat eine stärkere lineare Ausdehnung $\left(\alpha^{\prime}=46,5 \cdot 10^{-6} \mathrm{~K}^{-1}\right)$ als die typische Längenausdehung der Metalle Gold (oder Eisen $\alpha_{\mathrm{Fe}}=12,2 \cdot 10^{-6} \mathrm{~K}^{-1}$ ). Dies weist auf Zugspannungen in den Schichtpaketen hin. Diese Beobachtung gilt analog für Eisen/GoldSchichtsysteme auf facettiertem $\mathrm{Al}_{2} \mathrm{O}_{3}$, das horizontal beschichtet ist $\left(\alpha_{\text {facet.,horizontal }}^{\prime}=48,7 \cdot 10^{-6} \mathrm{~K}^{-1}\right)$. Im Fall der vertikalen, präferenziellen Beschichtung wird mit $\alpha_{\text {facet.,vertikal }}^{\prime}=27,0 \cdot 10^{-6} \mathrm{~K}^{-1}$ die niedrigste lineare Längenausdehung gemessen. Im Zustand nach der Herstellung liegt in diesem System aber auch die geringste Kompression der Abstände der Gold (111)-Ebenen gegenüber dem Gleichgewichtswert vor. Da der Gold (111)-Netzebenenabstand vor der Temperung 0,3-0,8\% unter dem Wert für Kompaktmaterial liegt, kann die Relaxation in die Gleichgewichtsabstände zu einer verstärkten Ausdehnung während des Heizvorgangs führen. Bei Erreichen der Zerfallstemperatur des Schichtaufbaus kommt es zu einer Abweichung vom vorausgehenden linearen Ausdehnungsverhalten.

Nach der Temperung treten keine Anteile anderer Orientierung im gesamten Röntgenspektrum auf (Abbildung 132), auch Eisen ist nicht detektierbar.

Qualitativ zeigt sich, bei insgesamt niedrigerer Intensität für das System auf facettiertem Substrat bei horizontaler Facettierungsausrichtung während der Depositon ein sehr ähnliches Bild (siehe Abbildung 129). Ein Vergleich der Intensitätsentwicklungen der

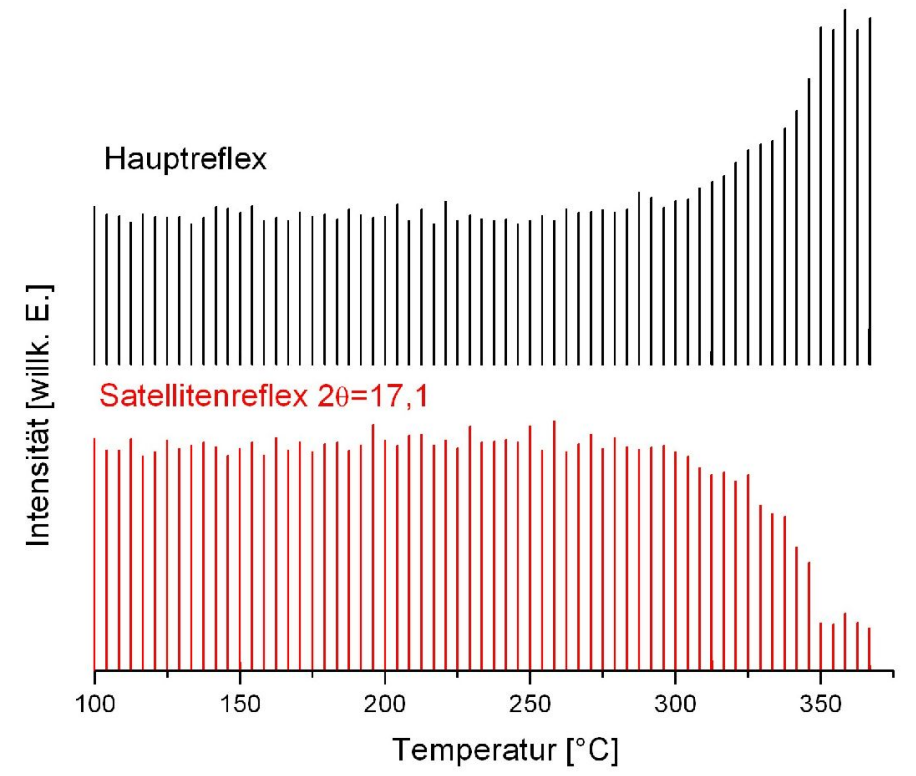

Abbildung 133: Intensitätsverlauf von Haupt- und Satellitenreflex, $50 x(2 \mathrm{~nm} \mathrm{Fe} / 10 \mathrm{~nm} \mathrm{Au})$ auf facettiertem $\mathrm{Al}_{2} \mathrm{O}_{3}$, horizontal beschichtet.
Eisen/Gold-Schichtsystem auf glattem (siehe Abbildung 130a) und facettiertem, homogen beschichteten Substrat (siehe Abbildung 133) verdeutlicht, dass der Zerfall des Schiichtaufbaus im gleichen Temperaturintervall von $275^{\circ}$ bis $350^{\circ} \mathrm{C}$ stattfindet. In diesem Temperaturbereich zeigt sich im beobachteten Winkelbereich eine weitere deutliche Veränderung. Aus einem sehr flachen Maximum bei $2 \Theta=19,9^{\circ}$ entwickelt sich bei der Temperatur, bei der die Vielfachschicht zerfällt, ein deutlicher, zum $\mathrm{Au}(111)$ Reflex vergleichbar breiter Reflex bei $2 \Theta=20,1$. Die Kohärenztiefe zeigt einen stufenartigen Anstieg zwischen $338^{\circ} \mathrm{C}$ und $352^{\circ} \mathrm{C}$ für beide Reflexe, den $\mathrm{Au}$ (111)-Reflex und den Reflex bei $20,1^{\circ}$ 


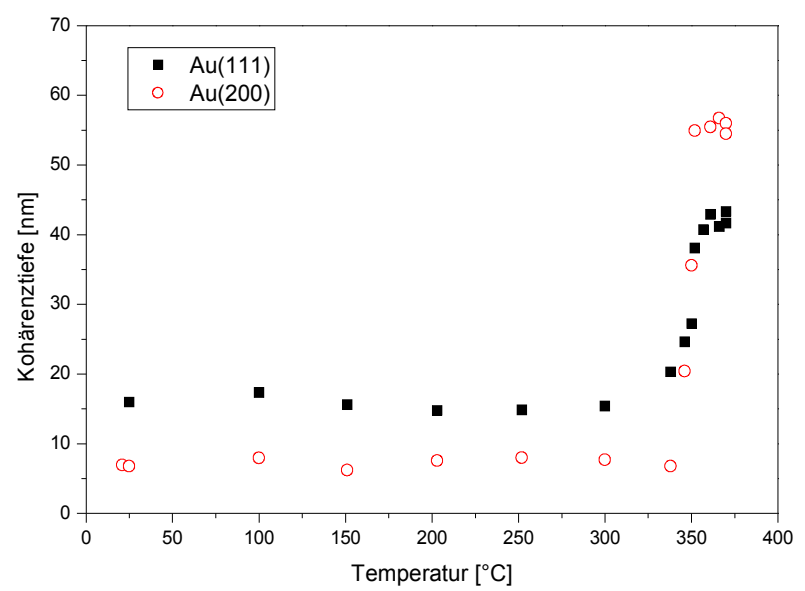

Abbildung 134: Kohärenztiefe ermittelt aus Au(111)- und Au(200)-Reflex während der Temperung eines $50 x(2 \mathrm{~nm} \mathrm{Fe} / 10 \mathrm{~nm} \mathrm{Au}$ )-Schichtsystems auf facettiertem $\mathrm{Al}_{2} \mathrm{O}_{3}$; horizontal beschichtet.

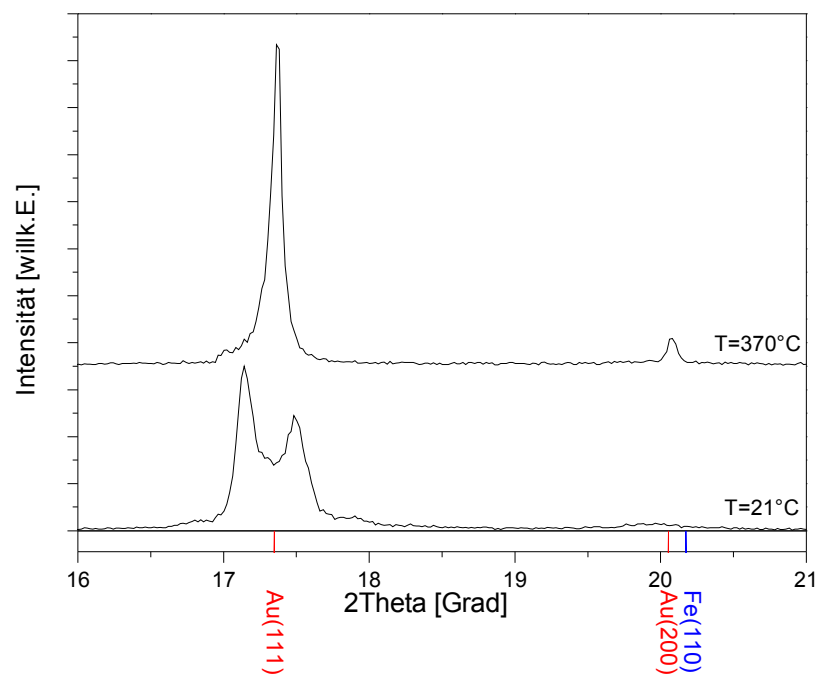

Abbildung 135: XRD-Diffraktogramme vor und nach der Temperung eines $50 x(2 \mathrm{~nm} \mathrm{Fe} / 10 \mathrm{~nm} \mathrm{Au})$-Schichtsystems auffacettiertem $\mathrm{Al}_{2} \mathrm{O}_{3}$; horizontal beschichtet.

(siehe Abbildung 134). Die eindeutige Zuordnung dieses Reflexes ist insofern schwierig, als dass $\mathrm{Au}(200)$ und $\mathrm{Fe}(110)$ mit $2 \Theta=20,05^{\circ}$ und $20,17^{\circ}$, wie in der Vergleichsdarstellung vor und nach dem Zerfall Abbildung $135 \mathrm{zu}$ sehen, sehr nahe beieinander liegen. Dies entspricht auch vorausgegangenen Beobachtungen in Eisen/Silber- Schichtsystemen, bei denen die Eisen(110)Komponente in Röntgenspektren im Temperaturbereich bis $350^{\circ} \mathrm{C}$ nicht von der $\operatorname{Ag}(200)$ Komponente zu trennen war [Her 99]. Die hohe Kohärenztiefe deutet jedoch darauf hin, dass der Reflex der $\mathrm{Au}(200)$-Orientierung zuzuordnen ist.

Die Intensitätszunahme des (111)-Hauptreflexes ist deutlich geringer als bei dem Vielfachschichtsystem auf glattem Substrat. Hier deutet sich an, dass im Bereich der Täler und Spitzen der Facetten, für den das Verfahren durch die Messung in Richtung der Gesamtsubstratnormalen sensitiv ist, bei der Temperung und dem Zerfall des Schichtaufbaus neben dem Wachstum bereits vorhandener (111)-Körner auf den Facetten insbesondere die dort vorhandenen stark gestörten Bereich rekristallisieren und - unter anderem - (200)-Körner bilden.

Die Analyse der Daten der in-situ Röntgenmessungen während der Temperung von Eisen/GoldSchichtsystemen auf facettiertem Saphir, dessen Facettenseiten durch den vertikalen Einbau bei der Beschichtung präferenziell belegt sind, zeigt neben dem Zerfall des Schichtsystems, wie schon zuvor an den anderen Konfigurationen diskutiert, einen ungewöhnlichen Verlauf der Intensität des Hauptreflexes. Bemerkenswert ist, dass dieser im Gegensatz zu den Beobachtungen auf den anderen Substrattypen bzw. -beschichtungsanordnungen (Abbildung 129a und b) durch den Zerfall des Schichtsystems nicht an Intensität gewinnt, vielmehr wird eine Reduktion der Intensität beobachtet (siehe Abbildung 129c). Wie die temperaturabhängigen Auftragungen der Intensitäten von Hauptund Satellitenreflex (Abbildung 136) zeigen, sinkt die Intensität des Satellitenreflexes bei $2 \Theta=17,1^{\circ}$ zwischen $287^{\circ} \mathrm{C}$ und $336^{\circ} \mathrm{C}$ deutlich ab, der Hauptreflex verliert im gleichen Temperaturintervall auch an Intensität und wandert zu kleineren Winkeln in die Kristallreflexlage. 


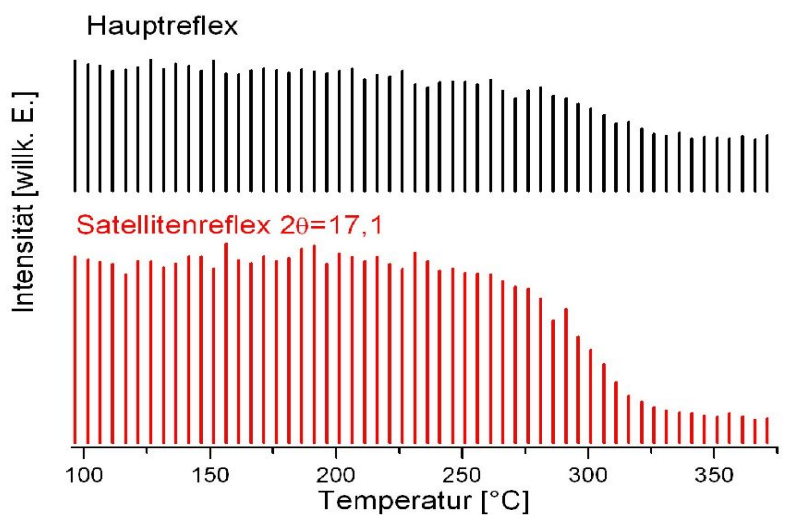

Abbildung 136: Abfall der Intensität bei Haupt- und Satellitenreflex, 50x(2nm Fe/10nm Au) Schichtsystem auf facettiertem $\mathrm{Al}_{2} \mathrm{O}_{3}$; vertikal beschichtet.

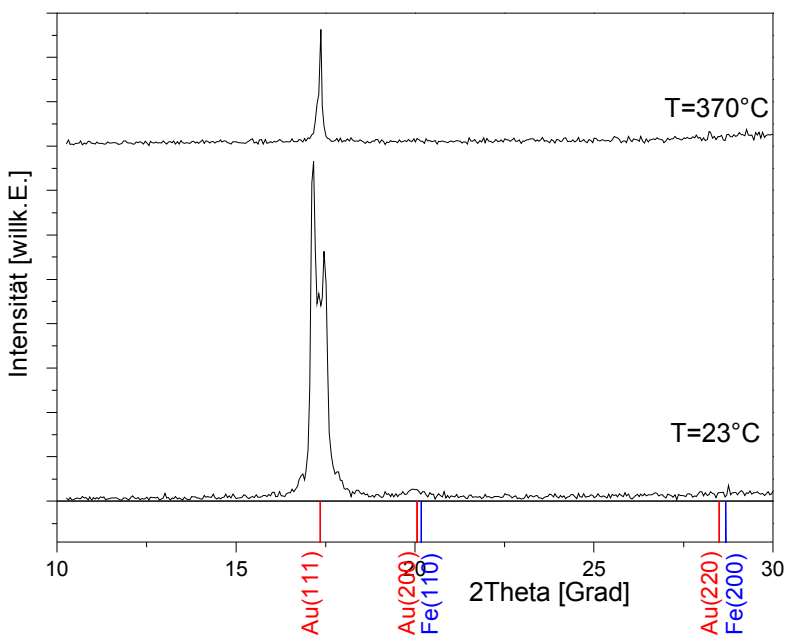

Abbildung 137: XRD-Diffraktogramme vor und nach der Temperung eines $50 x(2 \mathrm{nmFe} 10 \mathrm{~nm} \mathrm{Au})$ Schichtsystems, auf facettiertem $\mathrm{Al}_{2} \mathrm{O}_{3}$; vertikal beschichtet.

Nach der Temperung ist im gesamten gemessenen Winkelbereich von $10^{\circ}$ bis $30^{\circ}$ kein weiterer Reflex vorhanden (siehe Abbildung 137).

Einen Erklärungsansatz für die ungewöhnliche Intensitätsentwicklung im Fall vertikal ausgerichteter Facetten bei der Beschichtung liefern Texturanalysen im wie-hergestellten Zustand und nach der Temperung in Abbildung 138. Die (111)-Polfiguren zeigen, dass bei diesen Schichtsystemen mit präferentiellen Belegung der Facettenseiten durch eine Temperung eine besonders starke Verschärfung der Textur beobachtet wird. Nach der Herstellung ist noch ein deutliches Maximum im Zentrum der Polfigur von Abbildung 138a zu erkennen, obgleich die Intensität des Maximums einer Facettenseite höher ist. Nach der Temperung ist im Zentrum keine wesentlich vom Untergrund verschiedene Intensität nachweisbar, während das Maximum auf der Facettenseite verstärkt ist. Dementsprechend liegt es nahe, dass Bereiche, die eine (111)Ausrichtung bzgl. der Gesamtsubstratnormalen aufweisen, in einem Rekristallisationsprozess während der Temperung durch Wachstum der bezüglich der Facettenseite (111)-orientierten Körner aufgezehrt werden. Wie die mikrostrukturellen Untersuchungen gezeigt haben, finden sich solche
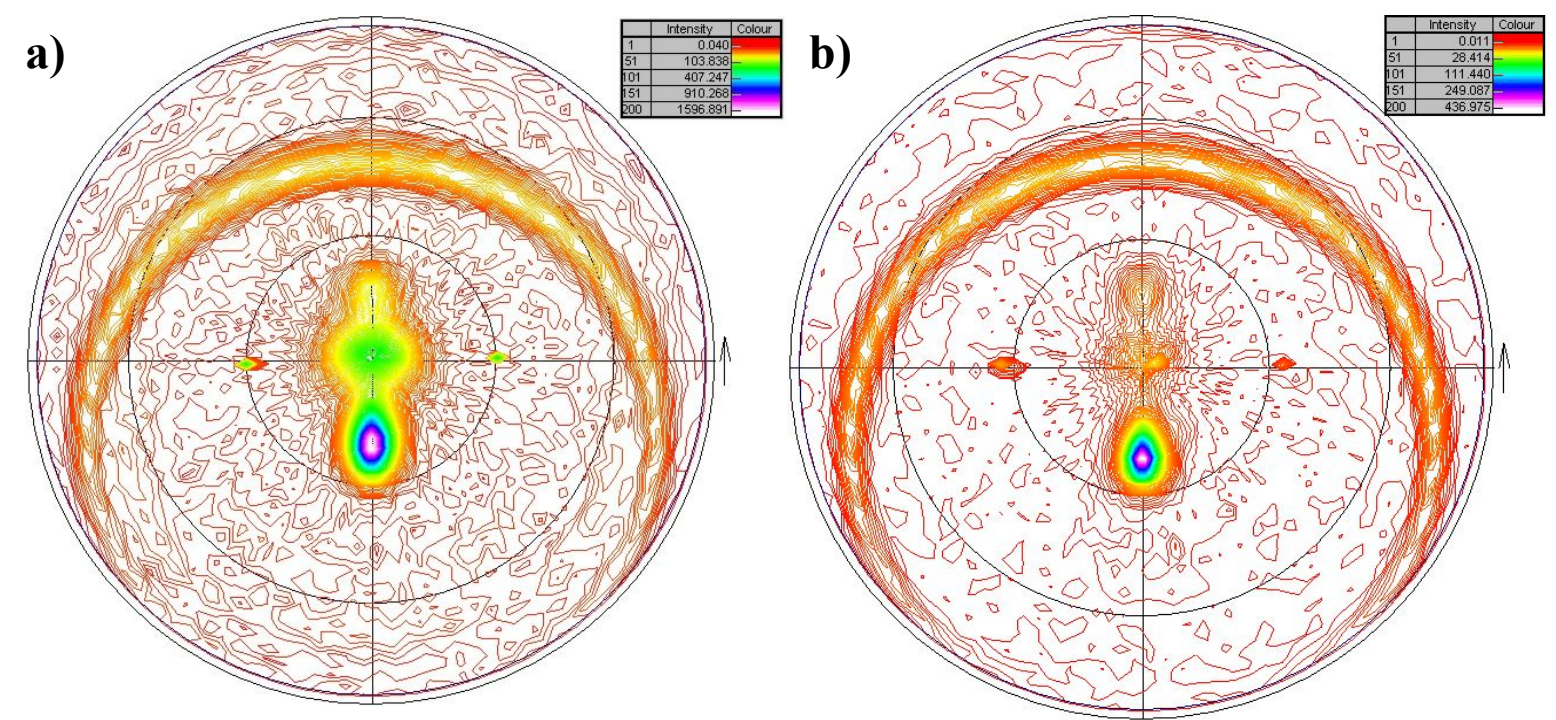

Abbildung 138: Texturanalyse bei Au(111)-Reflexbedingung eines 50x(2nm Eisen/10nm Gold) Schichtsystems, auf facettiertem $\mathrm{Al}_{3} \mathrm{O}_{3}$; vertikal beschichtet; a) wie hergestellt und b) nach 60 min bei $370^{\circ} \mathrm{C}$. 
Bereiche auf den Facettenkämmen und sind sehr defektreich. Es ist bekannt, dass die Kristallisationstemperatur im Bereich von wenigen Nanometern einen Größeneffekt zeigt, d.h. die Schmelztemperatur nahezu linear proportional zum reziproken Teilchendurchmesser ist [All 86]. Die starke Störung der Mikrostruktur ist daher auch notwendig, um Rekristallisation bei den vergleichsweise niedrigen Temperaturen von ca. $350^{\circ} \mathrm{C}$ überhaupt möglich erscheinen zu lassen.

Ein Vergleich von Abbildung 132, Abbildung 135 und Abbildung 137 verdeutlicht die unterschiedliche Intensitätsentwicklung durch die Temperung für die unterschiedlichen Beschichtungsanordnung während der Deposition der Schichtsysteme.

Eine zwölffache Überhöhung der Daten eines Eisen/Silber-Schichtsystems gegenüber dem Eisen/Gold System ist notwendig für die vergleichende Darstellung vor der Temperung in Abbildung 139. Man erkennt eine wesentlich schlechter ausgeprägte Überstruktur im Vergleich zu Eisen/Gold, was die Untersuchung für die Proben mit Silber als Edelmetallkomponente erschwert.

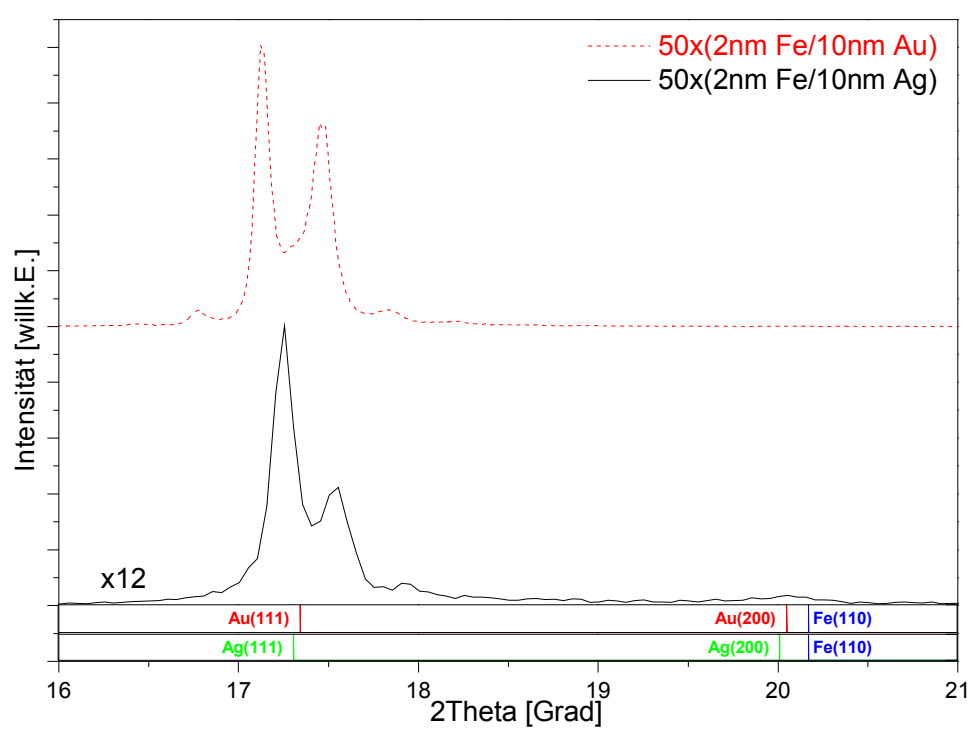

Abbildung 139: XRD-Diffraktogramme: 12-fach geringere Intensität von gleichwertigen Eisen/Silber-gegenüber Eisen/Gold-Schichtsystemen auf glattem Substrat, wie hergestellt.

Dennoch ließen sich Daten wie die

Zerfallstemperatur aus den temperaturabhängigen Diffraktogrammen bestimmen, unter Einbezug der Veränderungen von Kohärenzlänge und Ausdehnungsverhalten in die Auswertung.

Abbildung 140 zeigt die Entwicklung des Netzebenenabstands der Silber(111)-Ebenen während des Temperprozesses des Eisen/Silber-Schichtsystems auf glattem $\mathrm{Al}_{2} \mathrm{O}_{3}$ bis $400^{\circ} \mathrm{C}$. Nach einem Bereich linearer thermischer Ausdehnung von Raumtemperatur bis ca. $300^{\circ} \mathrm{C}$ stagniert die Vergrößerung der Gitterkonstante. Im entsprechenden Temperaturbereich kompensieren sich die

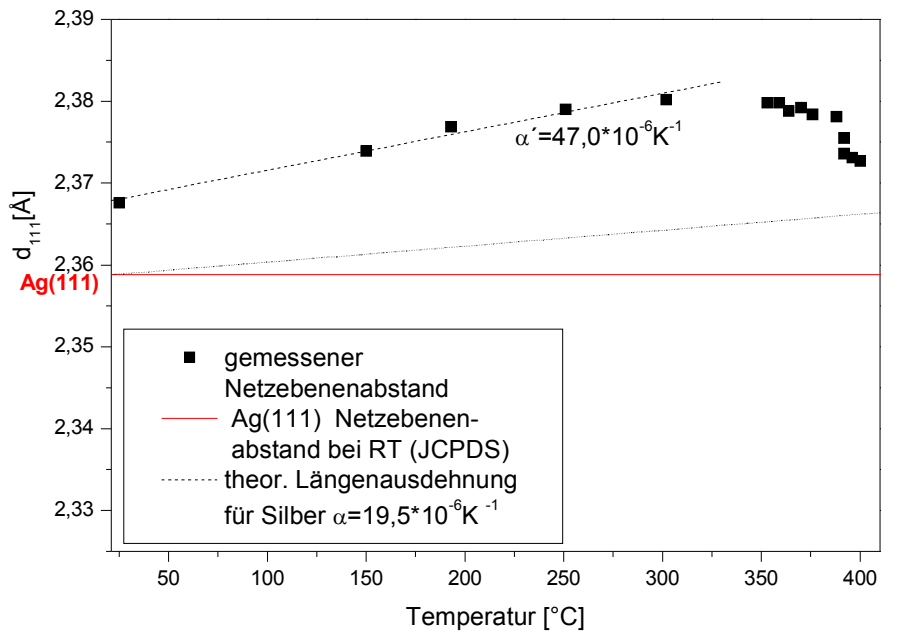

Abbildung 140: Ag(111)-Netzebenenabstand, $50 x(2 \mathrm{~nm} \mathrm{Fe} / 10 \mathrm{~nm} \mathrm{Ag})$-Schichtsystem auf glattem $\mathrm{Al}_{2} \mathrm{O}_{3}$. thermische Ausdehnung und die Verlagerung in Richtung des idealen Netzebenenabstands. Zwischen $388^{\circ} \mathrm{C}$ und $396^{\circ} \mathrm{C}$ kommt es $\mathrm{zu}$ einer stufenartigen Reduktion. Dieses Intervall kann mit der vollständigen Zerstörung des Schichtaufbaus identifiziert werden. Im Gegensatz zu den Eisen/Gold-Schichtsystemen zeigt die Auswertung der Kristallreflexlagen bei Eisen/Silber, dass die Gitterzelle des Edelmetalls in Richtung der Substratnormalen gedehnt ist, was auf Druckspannungen in diesen Schichten hinweist. Abbildung 99 hat bereits gezeigt, dass besonders im System Eisen/Silber der Silber(200)-Anteil in den Diffraktogrammen gegenüber dem (111)-Anteil bei Deposition auf den facettierten Substraten 
deutlich steigt. Es ist bei diesen Proben also auch möglich, diesen für die Bestimmung der Zerfallstemperatur heranzuziehen, da der Einfluss des Übergitters auf die Intensitätsverteilung um den Silber (111)-Kristallreflex deutlich gemindert ist. Abbildung 141 zeigt daher die Temperaturentwicklung der Netzebenenabstände für die (111)- und (200)-Anteile eines Eisen/Silber-Schichtsystems auf vertikal ausgerichtet beschichtetem, facettiertem Saphir. Im niedrigen Temperaturbereich von Raumtemperatur bis $300^{\circ} \mathrm{C}$ spiegeln die Positionen beider Reflexe die thermische Ausdehnung wider.

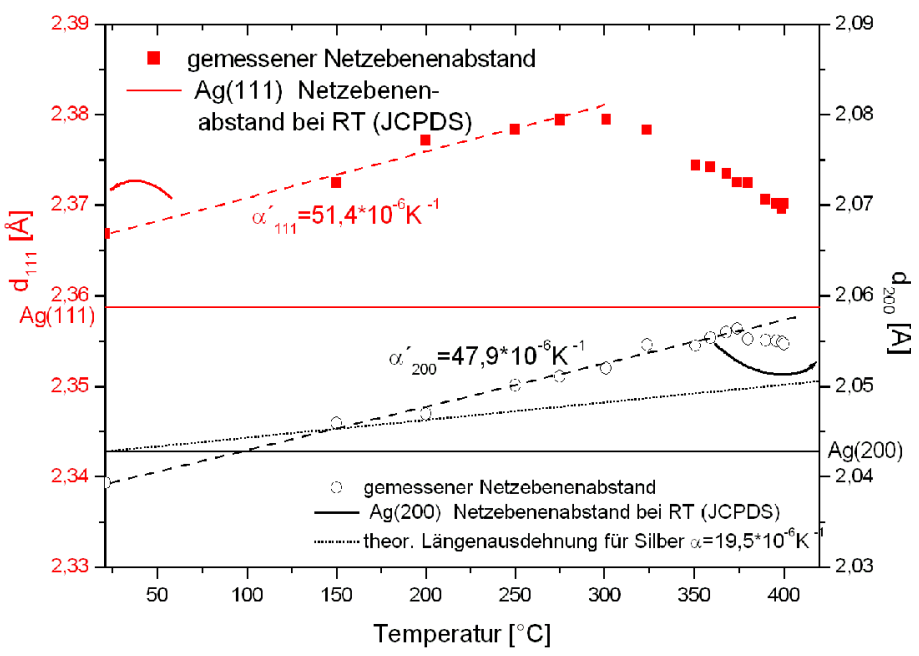

Abbildung 141: Entwicklung der Netzebenenabstände in $50 x(2 \mathrm{~nm} \mathrm{Fe} / 10 \mathrm{~nm} \mathrm{Ag})$ auf facettiertem $\mathrm{Al}_{2} \mathrm{O}_{3}$; vertikal beschichtet. Diese liegt jedoch mit $\alpha^{\prime} \approx 50 \cdot 10^{-6} \mathrm{~K}^{-1}$ ebenso wie bei allen anderen Proben deutlich über den tabellierten Werten für die lineare Längenausdehnungskoeffizienten [Dax 03] des Edelmetalls von $\alpha_{\mathrm{Ag}}=19,5 \cdot 10^{-6} \mathrm{~K}^{-1}$. Für die Werte aus dem (111)-Reflex folgt bei $300^{\circ} \mathrm{C}$ ein Absinken, während für die Gitterebenen mit (200)-Wachstumsrichtung erst bei $365^{\circ} \mathrm{C}$ ein Abweichen vom linearen Anstieg sichtbar wird.

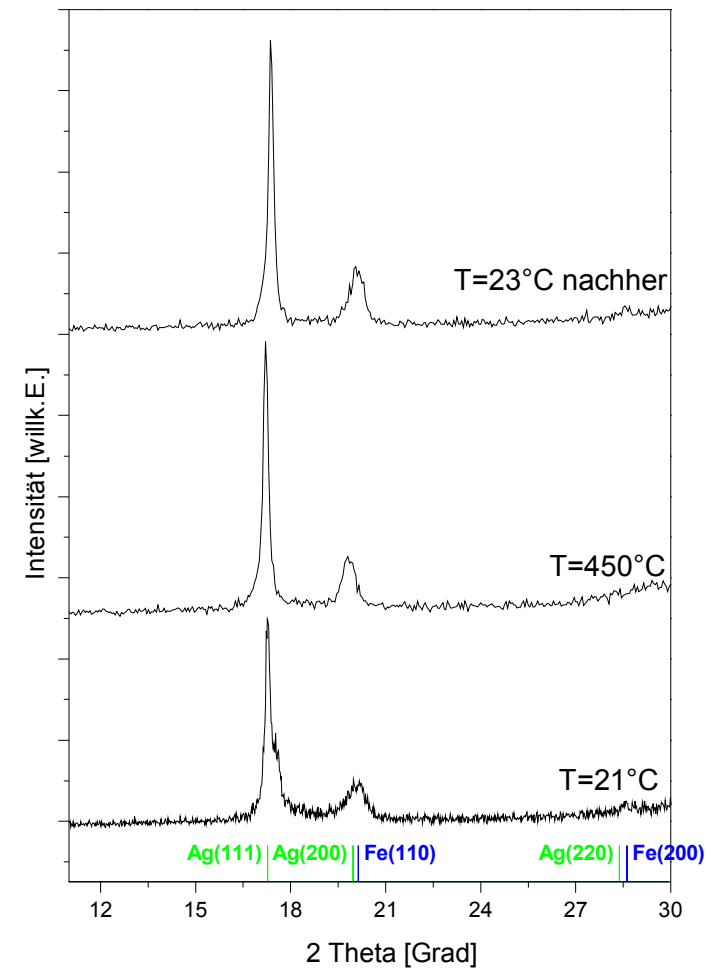

Abbildung 142: geringe Veränderung der XRD-Spektren durch Temperung eines 50x(2nm Fe/10nm Ag)-Schichtsystems auf facettiertem $\mathrm{Al}_{2} \mathrm{O}_{3}$; horizontal beschichtet.
Die ermittelte Kohärenztiefe zeigt ebenfalls bei Temperaturen größer als $350^{\circ} \mathrm{C}$ einen signifikanten Anstieg.

Somit bestätigen also auch die Untersuchungen mittels Röntgenbeugung am Synchrotronlabor, dass die Zerfallstemperatur des Schichtaufbaus im thermisch stabileren System Eisen/Silber durch die Aufbringung auf ein facettiertes Substrat um mindestens $20-30^{\circ} \mathrm{C}$ abgesenkt werden kann.

Für die bei der Deposition des Eisen/Silber-Schichtpakets horizontal eingebauten Saphirsubstrate sind die Veränderungen durch die Temperung am geringsten (siehe Abbildung 142).

Das Abweichen vom linearen thermischen Ausdehnungsverhalten oberhalb von $\sim 350^{\circ} \mathrm{C}$ kann als untere Grenze für den Beginn des Zerfalls des Übergitters gesehen werden.

Zusammenfassend kann festgestellt werden, dass im System Eisen/Gold ein ausgeprägtes (111)-Wachstum auf den Facetten sowie ein Wachstum dieser Körner und Ausheilen von Defekten während der Temperaturbehandlung erfolgt. Die Modulation der Intensitätsverteilung in den Diffraktogrammen durch das künstliche Übergitter ist auch auf den facettierten 
Substraten umfassend auswertbar, eine wesentliche Veränderung der Zerfallstemperatur von der Temperatur auf glatten Substraten ist diesen Daten nicht zu entnehmen. Die vollständige Zerstörung des Schichtsystems erfolgt bis zur Temperatur von $350^{\circ} \mathrm{C}$.

Die geometrischen Verhältnisse durch die Facettierung des Substrates behindern das Aufwachsen von Ebenen, deren Normalenvektor parallel zur Gesamtsubstratnormalen orientiert ist. Da das Verfahren der Röntgendiffraktometrie in Bragg-Brentano Geometrie aber für diese Richtung sensitiv ist, ist eine starke Verringerung der Intensität die Folge. Dies erschwert insbesondere die Beobachtung der thermischen Veränderungen der Eisen/Silber-Schichtsysteme, zusätzlich zu ihrem höheren polykristallinen Anteil und der schlechteren Kohärenz des Übergitters. Dabei hat der geringe $\operatorname{Ag}(111)$-Anteil im sensitiven Bereich der Spitzen und Täler offensichtlich negativen Einfluss auf diese Kohärenz.

Dennoch ist es durch umfangreiche Analyse der Daten gelungen, aufzuzeigen, dass die verbleibende, gegenüber dem Eisen/Gold deutlich gestörtere, Vielfachschichtstruktur beeinflusst durch die facettierte Unterlage schon bei tieferen Temperaturen zerfällt, als dies auf glatten Substraten der Fall ist. In-situ Leitfähigkeitsmessungen zeigen durch einen sprunghaften Anstieg der Leitfähigkeit die Frühstadien der Gestaltumwandlung schon bei niedrigeren Temperaturen an. So konnte beispielsweise für das System Eisen/Silber ein Einsatzpunkt bei $314^{\circ} \mathrm{C}$ bestimmt werden, obgleich auf glatten Substraten dieser erst bei $350^{\circ} \mathrm{C}$ auftritt und in-situ Röntgenmessungen konsistent mit TEM-Untersuchungen eine weitgehend erhaltene Lagenstruktur bis über $350^{\circ} \mathrm{C}$ hinaus bestätigen. Während in sämtlichen Eisen/Gold-Schichtsystemen kleinere (111)-Netzebenenabstände als im Kompaktmaterial gemessen wurden, tritt in den Eisen/Silber-Schichtsystemen eine Dehnung parallel zur Substratnormalen auf. In den damit verbundenen, unterschiedlichen Wachstumsbedingungen für die Edelmetalle mit Zug- bzw. Druckspannung kann eine Ursache für die bessere Röntgenkohärenz und die schärfere Textur gesehen werden.

Eine sehr hohe thermische Stabilität zeigt das Kohlenstoff/Kobalt-System. Vielfachschichten mit dreißig Doppellagen zeigen über den gesamten Großwinkelbereich trotz intensiver Strahlungsquelle keine kristallinen Reflexe. Abbildung 143a verdeutlicht, dass auch nach einer Temperung bis $600^{\circ} \mathrm{C}$ keine Veränderungen auftreten, lediglich der Substratreflex ist sichtbar. Dennoch zeigt das selbe Schichtsystem im Kleinwinkelbereich Bragg-Reflexe der künstlichen Übergitterstruktur (Abbildung 143b).
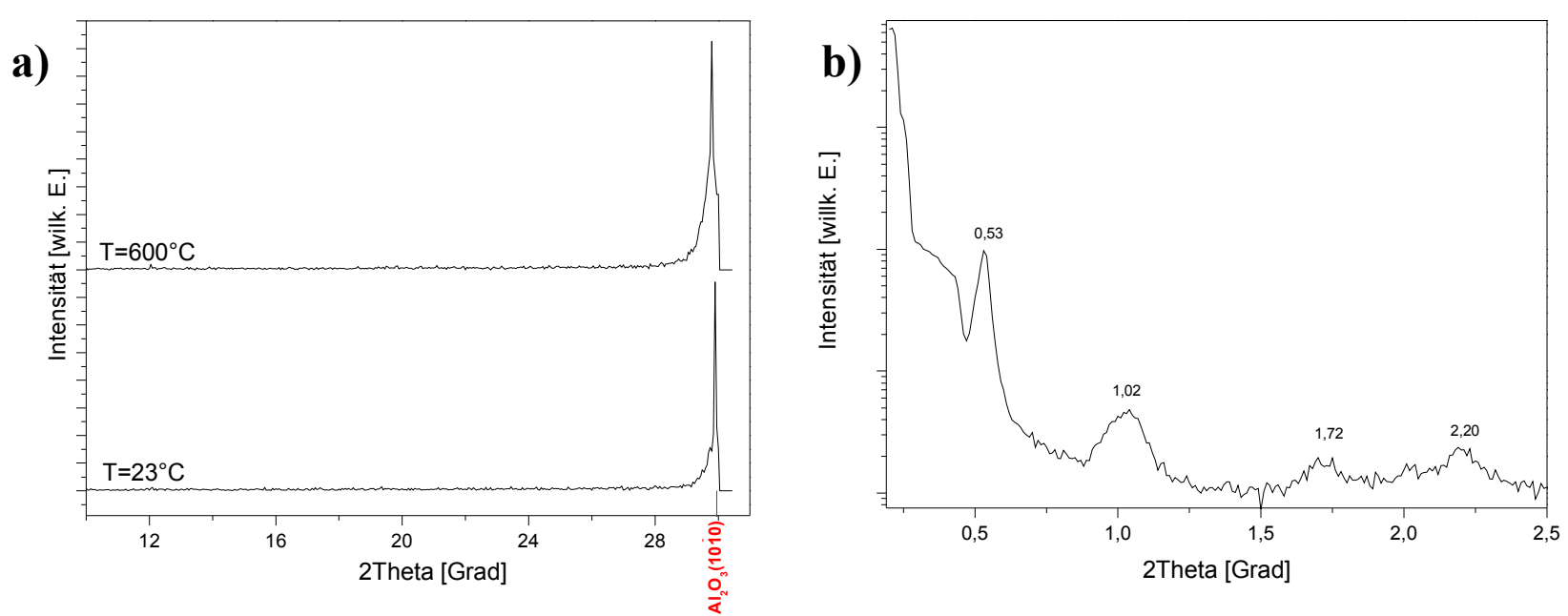

Abbildung 143: a) XRD-Spektren vor und nach der Temperung bis $600^{\circ} \mathrm{C}$ eines $30 x(2 \mathrm{~nm}$ Kobalt/10nm Kohlenstoff)Schichtsystems auffacettiertem Saphir, horizontal beschichtet.

b) zugehöriges XRD-Kleinwinkelspektrum. 
In Übereinstimmung mit den Ergebnissen der Feinbereichsbeugung an den Querschnittsproben bei den TEM Untersuchungen liegt der Kohlenstoff im Schichtsystem amorph vor. Auch Kobalt zeigt keine kristallinen Reflexe in den Diffraktogrammen oder im Beugungsmodus des Elektronenmikroskops. Die in-situ XRD-Messungen bis zu $600^{\circ} \mathrm{C}$ zeigen, dass weder Kobalt noch Kohlenstoff kristallisieren. Die große Dichtedifferenz der beiden Elemente des Schichtsystems ermöglicht aber eine hohe Reflektivität des künstlichen Übergitters bei kleinen Winkeln. In Zusammenhang mit den Ergebnissen zur Leitfähigkeitsmessung im Schichtsystem Kobalt/Kohlenstoff ist aber davon auszugehen, dass es zwischen $550^{\circ} \mathrm{C}$ und $600^{\circ} \mathrm{C}$ zu Veränderungen in der Lagenstruktur kommt, da die Leitfähigkeit in senkrechter Richtung zur Facettierung einbricht. Dabei muss offensichtlich davon ausgegangen werden, dass hier Verarmung von Kobalt an den Bereichen extremer Krümmung, nicht aber die Kristallisation wesentlicher Volumenbruchteile des Kobalts oder Kohlenstoffs Grund für die anisotrope Leitfähigkeit ist.

\subsubsection{Veränderungen von Gefüge und Mikrostruktur}

Neben den Veränderungen des inneren Aufbaus der Schichtsysteme, insbesondere dem Zerfall der Schichtstruktur, führt die Temperung der Schichtsysteme ebenso zu teilweise starken Änderungen im Korngefüge. Im Folgenden werden diese Änderungen im direkten Zusammenhang mit den mikrostrukturellen Veränderungen (sichtbar in den Texturanalysen) dargestellt und in Bezug auf die vorausgehenden Ergebnisse der Leitfähigkeitsveränderungen und in-situ Röntgenanalysen diskutiert.

Durch eine Temperung für $30 \mathrm{~min}$ bei $350^{\circ} \mathrm{C}$, bei der die Röntgenkohärenz des künstlichen Übergitters des Vielfachschichtsystems bereits verschwunden ist (siehe Abbildung 129), verändert sich die Oberflächenmorphologie nur wenig (Abbildung 144, vgl. Abbildung 74a, Seite 72 (wie hergestellt)). Grenzbereiche wirken schmaler. Einige Bereiche scheinen zusammengewachsen, so dass nun auch konkave Krümmungen in der Form einzelner Körner erkennbar sind.

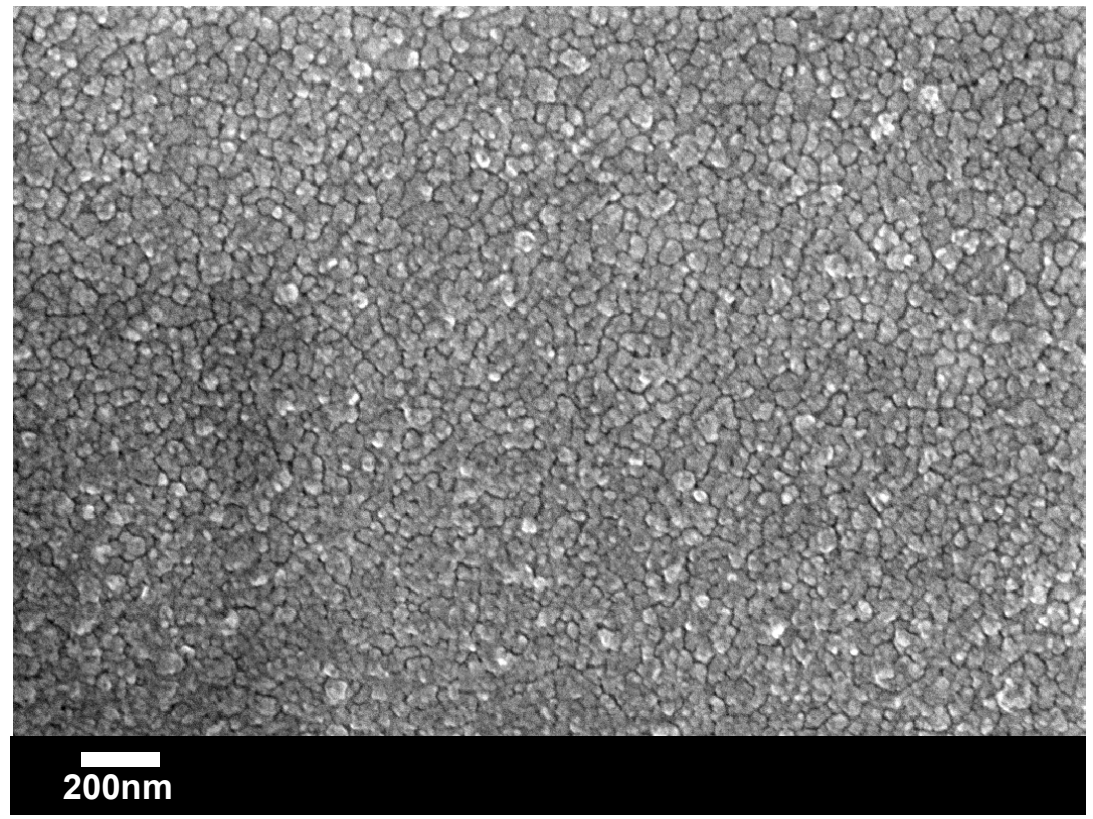

Abbildung 144: SEM-Abbildung: 50x(2nm Fe/10nm Au)-Schichtsystem, auf glattem $\mathrm{Al}_{2} \mathrm{O}_{3}, 30 \mathrm{~min}$ bei $350^{\circ} \mathrm{C}$ getempert. 


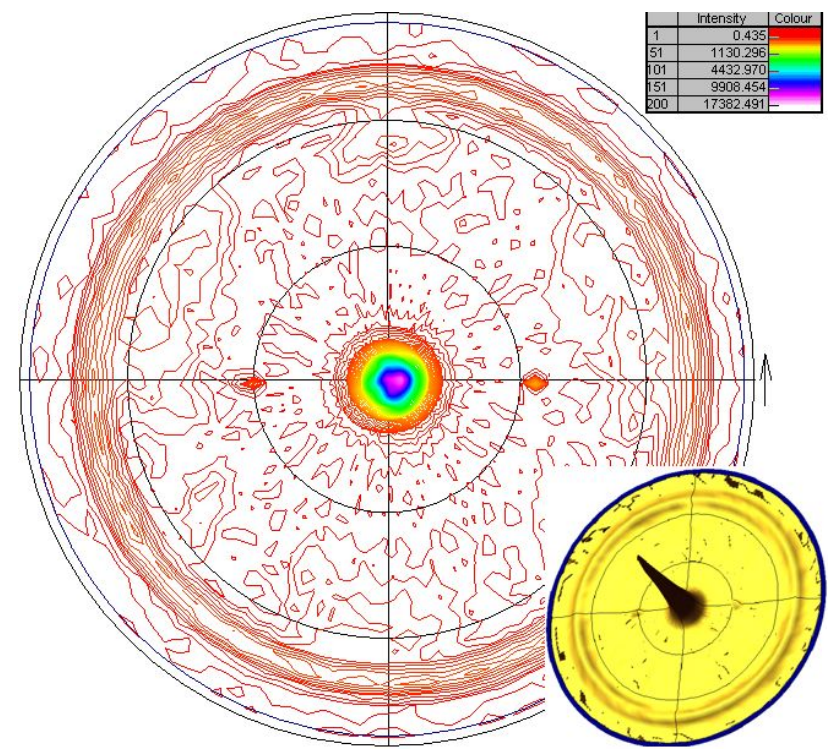

Abbildung 145: (111)-Polfigur eines

$50 x(2 \mathrm{~nm}$ Eisen/10nm Gold)-Schichtsystems

auf glattem $\mathrm{Al}_{2} \mathrm{O}_{3}, 30 \mathrm{~min}$ bei $350^{\circ} \mathrm{C}$ getempert.

Durch die Temperung verschärft sich die Textur. Der Abbau von Spannungen und Defekten hat eine höhere Intensität im Hauptmaximum und eine Verschmalerung des Reflexes zur Folge. Abbildung 145 zeigt die (111)-Polfigur eines $30 \mathrm{~min}$ bei $350^{\circ} \mathrm{C}$ getemperten Eisen/Gold-Schichtsystems. Die Intensität im zentralen Reflex der (111)-Ebenen parallel zur Substratoberfläche hat stark zugenommen, so dass der Ring unter $70,5^{\circ}$ schwächer erscheint. In der kleinen HalbraumDarstellung hebt sich dieser jedoch deutlich vom Untergrund $a b$.

Abbildung 146 zeigt die Oberfläche eines Eisen/Gold-Schichtpakets nach einer Temperung für $30 \mathrm{~min}$ bei $370^{\circ} \mathrm{C}$. Das Gefüge ist gegenüber der nicht getemperten (Abbildung 74b, Seite 72 )

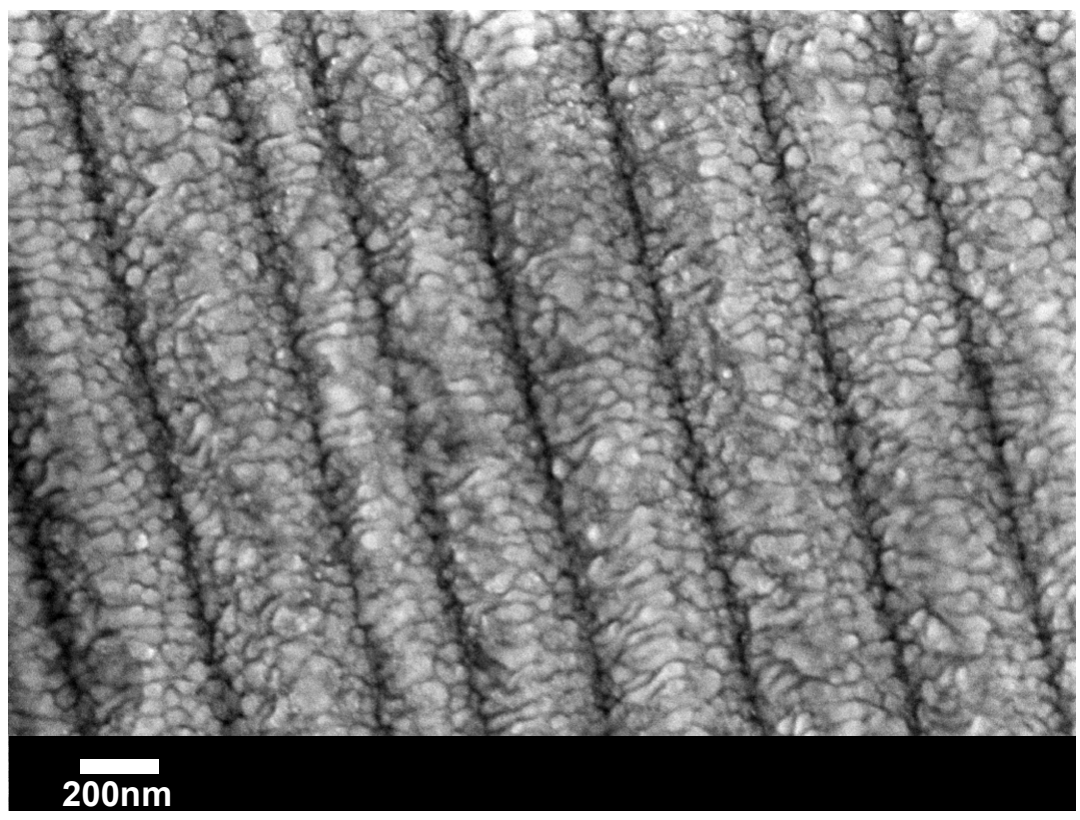

Abbildung 146: SEM-Abbildungen: 50x(2nm Fe/10nm Au)-Schichtsystem, auf facettiertem, horizontal beschichtetem $\mathrm{Al}_{2} \mathrm{O}_{3}$ für 30 min bei $370^{\circ} \mathrm{C}$ getempert. 
weitgehend unverändert, obwohl die inneren Grenzflächen des Schichtsystems zerstört sind, wie die röntgendiffraktometrischen Daten zeigen.

Abbildung 147 verdeutlicht die durch eine Temperung für $30 \mathrm{~min}$ bei $370^{\circ} \mathrm{C}$ hervorgerufenen mikrostrukturellen Veränderungen eines Eisen/Gold-Schichtpakets anhand von Texturanalysen. Die linke (111)-Polfigur (Abbildung 147a) zeigt ein in horizontaler Facettierungsrichtung gesputtertes Schichtpaket, so dass die Facetten vergleichsweise homogen belegt wurden, direkt nach der Herstellung, Abbildung 147b ein ebensolches nach 30min Temperung bei $370^{\circ} \mathrm{C}$. Der Vergleich der SEM-Abbildungen Abbildung 74b und Abbildung 146 zeigt noch keine wesentliche Veränderung der Oberflächenbeschaffenheit. Abbildung 147 zeigt jedoch, dass durchaus wesentliche mikrostrukturelle Veränderungen im Schichtsystem ablaufen. Die Intensitätsverteilung im Zentrum verschiebt sich deutlich. Während im wie hergestellten Zustand noch ein breites Maximum in der
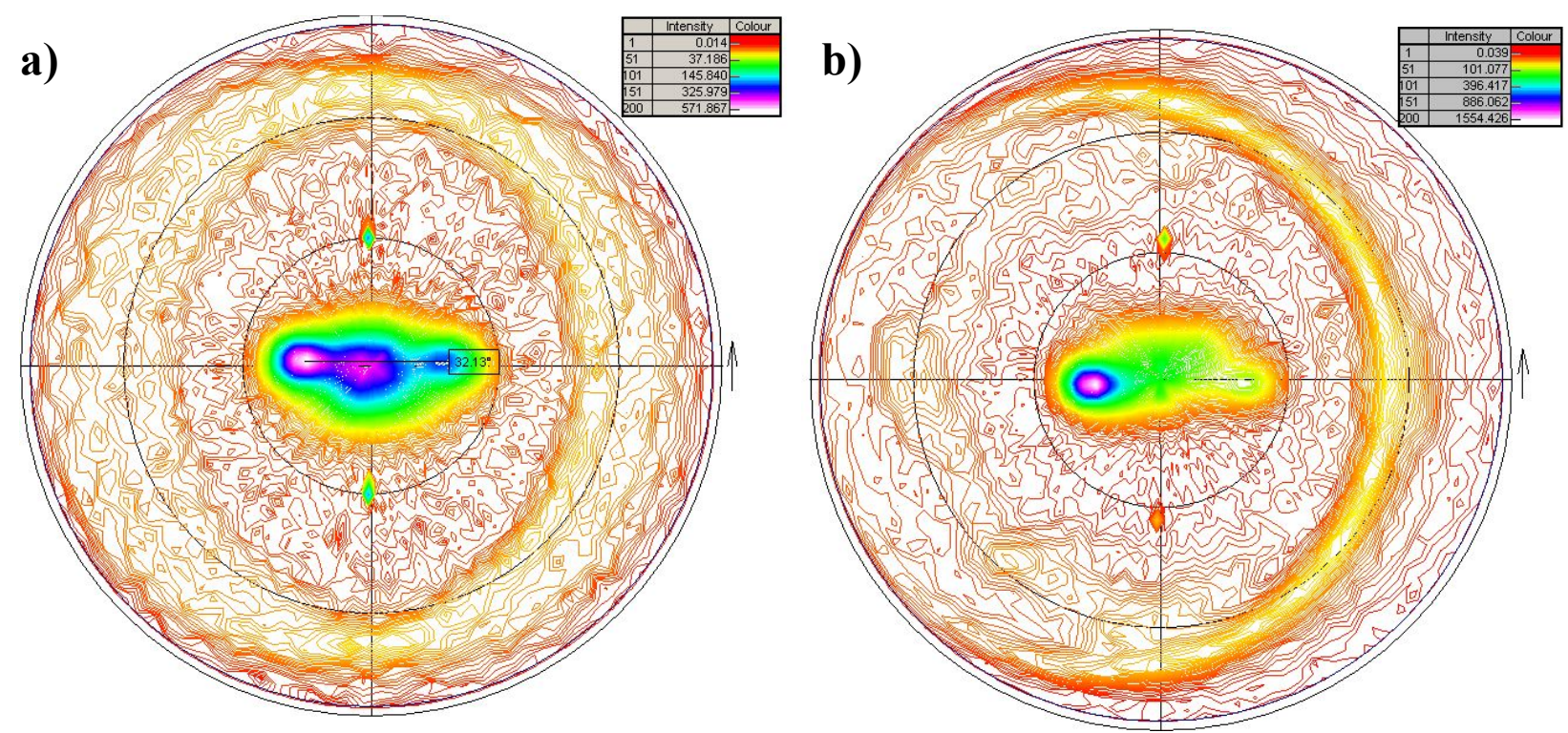

Abbildung 147: (111)-Polfigur eines 50x(2nm Eisen/10nm Gold)-Schichtsystem auf facettiertem $\mathrm{Al}_{2} \mathrm{O}_{3}$; horizontal beschichtet; a) wie hergestellt und b) nach $30 \mathrm{~min}$ bei $370^{\circ} \mathrm{C}$.

Mitte erkennbar ist, welches mit kleinen (111)-Goldkörnern in den defektreichen Zonen auf den Facettendächern und -tälern assoziiert werden kann, wird dieses durch die Temperung wesentlich geringer, wohingegen es zu einer Verschärfung der (111)-Goldreflexe auf den Facettenseiten kommt. Ebenso tritt der ringförmige Intensitätsverlauf unter $70,5^{\circ}$ erst durch die Temperung deutlich hervor.

Festzustellen ist, dass die rückgestreute Intensität für die beiden Facetten deutlich unterschiedlich ist, obwohl die Depositionsgeometrie keine der beiden Seiten bevorzugt. Dies deutet auf die unterschiedliche Beschaffenheit der beiden Facettenseiten hin, insbesondere da die SEM-Bilder und AFM-Daten keine wesentlichen Unterschiede aufgezeigt haben. Durch die Nanofacettierung der einen Facettenseite ist ein gleichmäßiges Wachstum gestört, was zu einer defektreichen Mikrostruktur von geringerer Röntgenkohärenztiefe führt.

Dass eine längere Temperung im Bereich der kritischen Zerfallstemperatur der Schichtsysteme auch deutliche Auswirkungen auf das Gefüge hat, zeigt die SEM-Abbildung 148. Durch die Verdoppelung der Temperungszeit sind an der Oberfläche starke Veränderungen zum Gefüge vor der Temperung erkennbar. Die laterale Periodizitätslänge, d.h. die Länge, auf der in der Darstellung senkrecht zur Facettierungsrichtung vergleichbare Muster auftreten, ist hier mit $\sim 150 \mathrm{~nm}$ nur halb so groß und kann mit den einzelnen Facettenseiten korreliert werden. Die Gefügetrennung durch die Facetten ist geringer, Bereiche auf benachbarten Facetten greifen an den Grenzen teilweise 

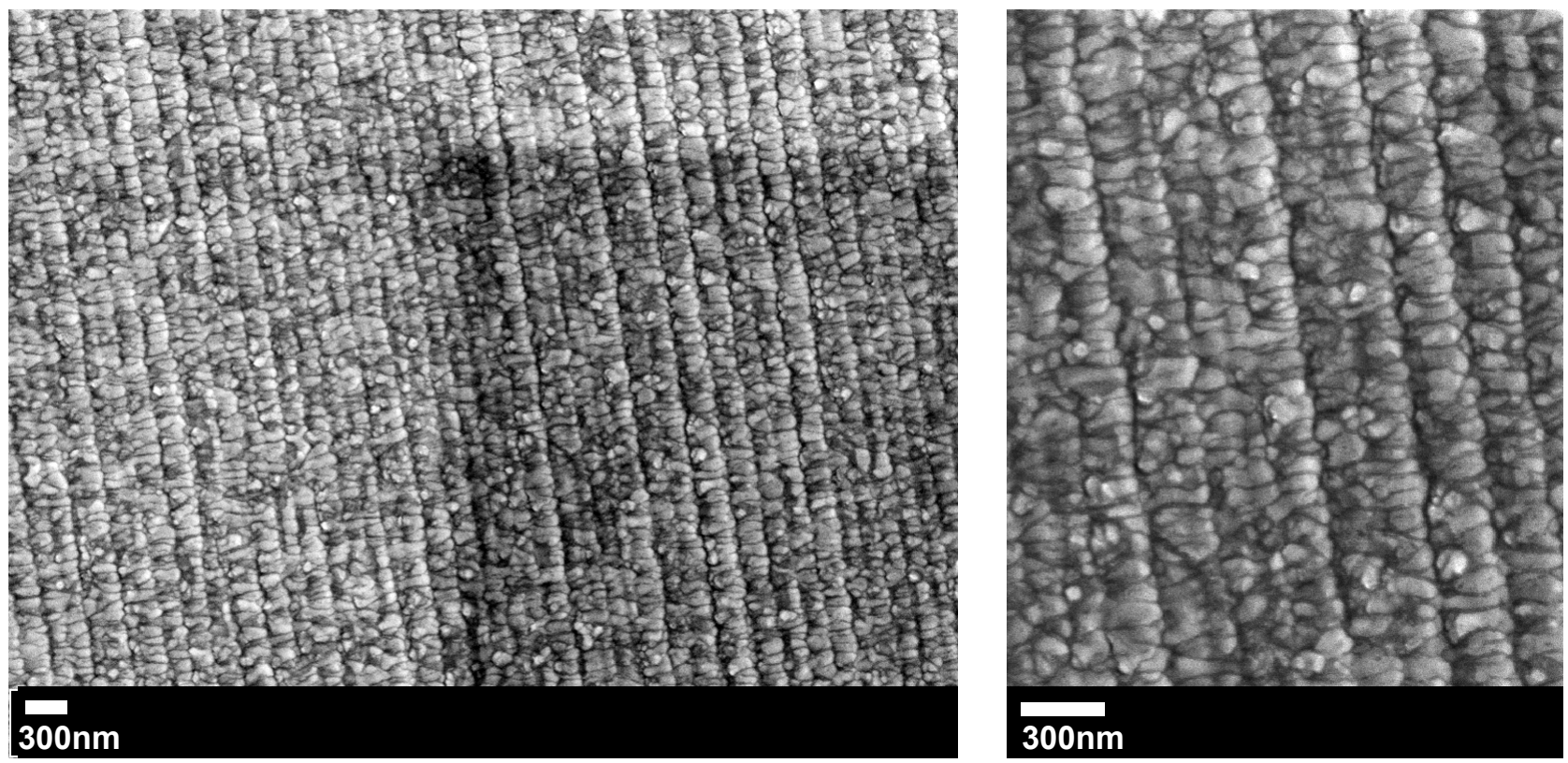

Abbildung 148: SEM-Abbildungen: 50x(2nm Eisen/10nm Gold)-Schichtsystem auffacettiertem $\mathrm{Al}_{2} \mathrm{O}_{3}$; vertikal beschichtet für $60 \mathrm{~min}$ bei $370^{\circ} \mathrm{C}$ getempert, Ausschnitt rechts doppelte Vergrößerung.

reißverschlussähnlich ineinander. Der Wachstumssäulendurchmesser ist senkrecht zur Facettierungsrichtung in diesem System durch die Breite einer Facette von 150nm begrenzt, es findet in seltenen Fällen ein Überwachsen von Facettenkämmen statt. Täler werden fast nie überwachsen. Die mittlere Ausdehnung der Säulen in Facettierungsrichtung beträgt $65 \mathrm{~nm}$, womit sich ein Aspektverhältnis von 2,3 ergibt. Es ist zu beachten, dass die Wachstumssäulen an der Oberfläche keine klare geometrische Form aufweisen.

Sowohl in Bereichen der Facettenkämme, als auch in den Tälern sind deutlich kleinere, sphärische Partikel mit einem Durchmesser von typisch $>40 \mathrm{~nm}$ (einige bis $\mathrm{zu} 60 \mathrm{~nm}$ ) zu finden. Dominiert wird das Gefüge von der Anpassung der lateralen Breite der Wachstumssäulen an die Facettenseitenbreite. Diese Rückschlüsse aus der Oberflächenanalyse bestätigt auch die Texturanalyse (Abbildung 149). Die Intensität

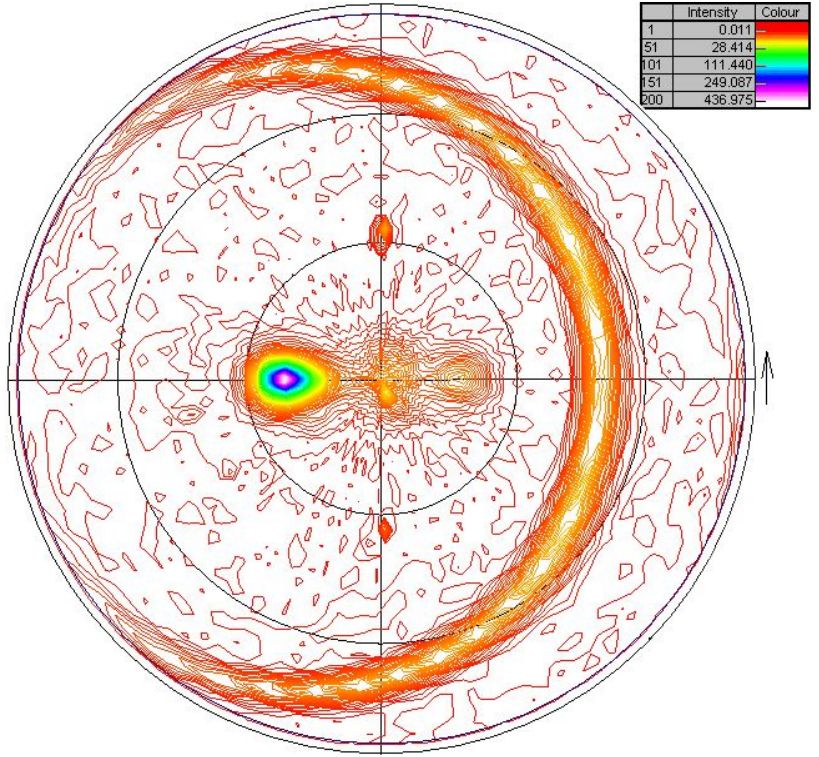

Abbildung 149: (111)-Polfigur eines $50 x\left(2 \mathrm{~nm}\right.$ Eisen $/ 10 \mathrm{~nm}$ Gold) nach $60 \mathrm{~min}$ bei $370^{\circ} \mathrm{C}$ (zu Abbildung 148). aus dem Zentrum ist fast vollständig $\mathrm{zu}$ Gunsten der auf den Facetten verschwunden. Die Gefügeänderung durch doppelte Temperzeit von Abbildung $146 \mathrm{zu}$ Abbildung 148 zeigt die Anpassung der Körner an die Facettenbreite, und das „Aufbrechen“ an den Facettenkämmen spiegelt sich in den Röntgenstrukturdaten wider.

Nach der Temperung wird bei der Oberflächenanalyse mittels SEM ein wesenticher Unterschied zwischen den Gefügen der Schichtsysteme Eisen/Silber bzw. Eisen/Gold deutlich. Während im System Eisen/Silber vergleichsweise runde Bereiche an der Oberfläche sichtbar sind (Abbildung 150), passen sich diese bei hinreichend getemperten Eisen/Gold-Proben vielfach der Breite der 


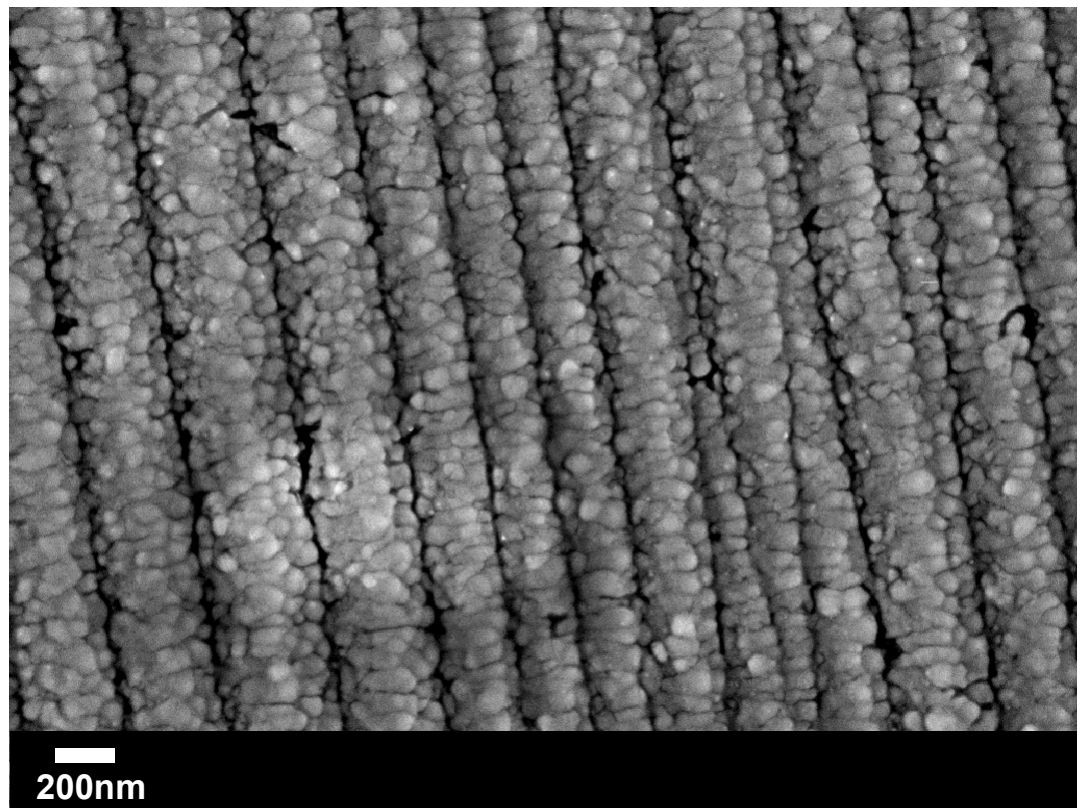

Abbildung 150: SEM-Abbildung: 50x(2nm Eisen/10nm Silber)-

Schichtsystem auffacettiertem $\mathrm{Al}_{2} \mathrm{O}_{3}$; vertikal beschichtet nach Temperung für $60 \mathrm{~min}$ bei $400^{\circ} \mathrm{C}$.

Facettenseiten (Abbildung 148) an. Dennoch sind nach der Temperung aber auch bei den Eisen/Silber-Schichtpaketen Rückwirkungen der Substratfacettierung auf das Gefüge erkennbar.

Ein solches Schichtpaket auf facettiertem Saphir getempert bis $400^{\circ} \mathrm{C}$ zeigt in Abbildung 150 „Streifenbreiten“, die einer gesamten Facette bestehend aus zwei Facettenseiten entsprechen. Körner überwachsen den Facettenkamm, wohingegen die Täler besonders starken dunklen Kontrast aufweisen. Wahrscheinlich sind nach der Temperung auch im System Eisen/Silber die Signaturen der Wachstumssäulen $\mathrm{zu}$ beobachten, die typischerweise in Eisen/Silber-Schichtsystemen größer sind als im System Eisen/Gold. Es konnte keine Stelle beobachtet werden, an der ein Tal überwachsen wurde, vielmehr separieren die Täler das Gefüge der benachbarten Facetten. Typische Bereichsgrößen sind hier 40-70nm und ein deutlich von eins verschiedenes Verhältnis der Halbachsen einer angenommenen ellipsodalen Form findet sich selten, dann aber vorzugsweise in Form von sehr großen Bereichen, die mittig auf den Facettenkämmen liegen mit Halbachsen von 220nm und 60nm entsprechend einem Verhältnis von 3,7. Dabei ist die große Halbachse immer nahezu senkrecht zur Facettierungsrichtung. Kleinere, runde Körner eines Durchmessers von $50 \pm 10 \mathrm{~nm}$ säumen die Gefügestreifen im Bereich der „Trennung“ in den Tälern. Auffällig ist auch, dass an Stellen, an denen die Facetten schmaler sind, bis zu einer Breite von ca. 120nm nur einzelne Wachstumssäulen auf diesen zu finden sind. Erst jenseits dieser Breite neigt das System dazu, unter Einbringung einer weiteren Grenze die Facettenbreite durch zwei oder mehr Wachstumssäulen zu überspannen. Für diese Beobachtungen eignen sich Bereiche, in denen die Facettierung des Substrats fehlerhaft ist, d.h. durch die Koaleszenz von Facettierungsdomänen versetzungsähnliche Fehler entstanden sind, da dort die

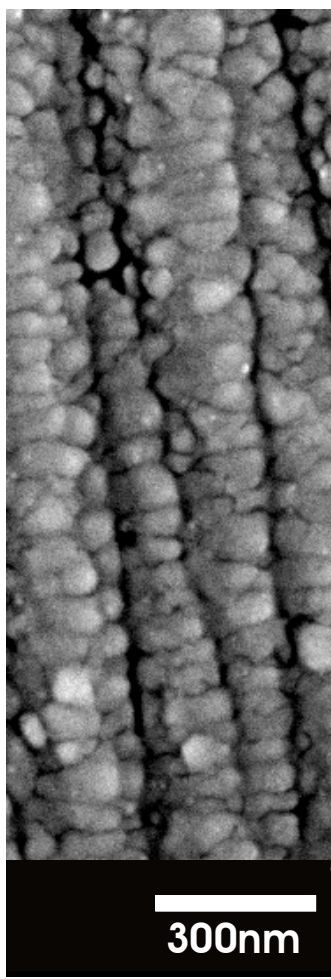

Abbildung 151: SEMAbbildung: Anpassung der Wachstumssäulenbreite an die Facettenseite. 
Facettenseitenbreite stetig bis zur Endbreite von ca. 150nm zunimmt. Das Gefüge eines solchen Bereichs nach Deposition und Temperung bis $400^{\circ} \mathrm{C}$ für Eisen/Silber zeigt Abbildung 151 in hoher Auflösung.Letztlich ist aber festzustellen, dass Eisen/Silber-Schichtsysteme ein inhomogeneres, ungerichteteres Gefüge an der Oberfläche zeigen als Eisen/Gold-Schichtsysteme.

Abbildung 152 zeigt (111)-Polfiguren von Eisen/Silber-Schichtsystemen nach der Temperung. Bei den Schichtsystemen mit Silber als Edelmetallkomponente wird der Unterschied zwischen Schichten mit homogener Bedeckung (Abbildung 152a) und denen präferentieller Beschichtung einer Facettenseite (Abbildung 152b) deutlicher als bei Eisen/Gold. Abbildung 152a ist nach einstündiger Temperung von bei $450^{\circ} \mathrm{C}$ entstanden und zeigt ein breites Maximum, das sich über einen Bereich von ca. $40^{\circ}$ erstreckt. Es ist senkrecht zur Facettierungsrichtung ausgedehnt. Die Temperung, obwohl mit $400^{\circ} \mathrm{C}$ bzw. $450^{\circ} \mathrm{C}$ bei höheren Temperaturen und langen Temperzeiten (1 Stunde) ausgeführt, verschärfen die Textur nicht in einer vergleichbaren Weise, wie beim Eisen/Gold beobachtet. Vielmehr zerfällt durch die Temperung der Ring von statistischen in-plane Orientierungen unter $\mathrm{psi}=70,5^{\circ}$ in Bereiche höherer Intensität (vgl. Abbildungen 105b und 152b). Die Abrundung des Reflexes maximaler Intensität in Abbildung 152b spricht für Veränderung des Gefüges mit Angleichung des Aspektverhältnisses zwischen Korngröße senkrecht und parallel zur Facettierungsrichtung.
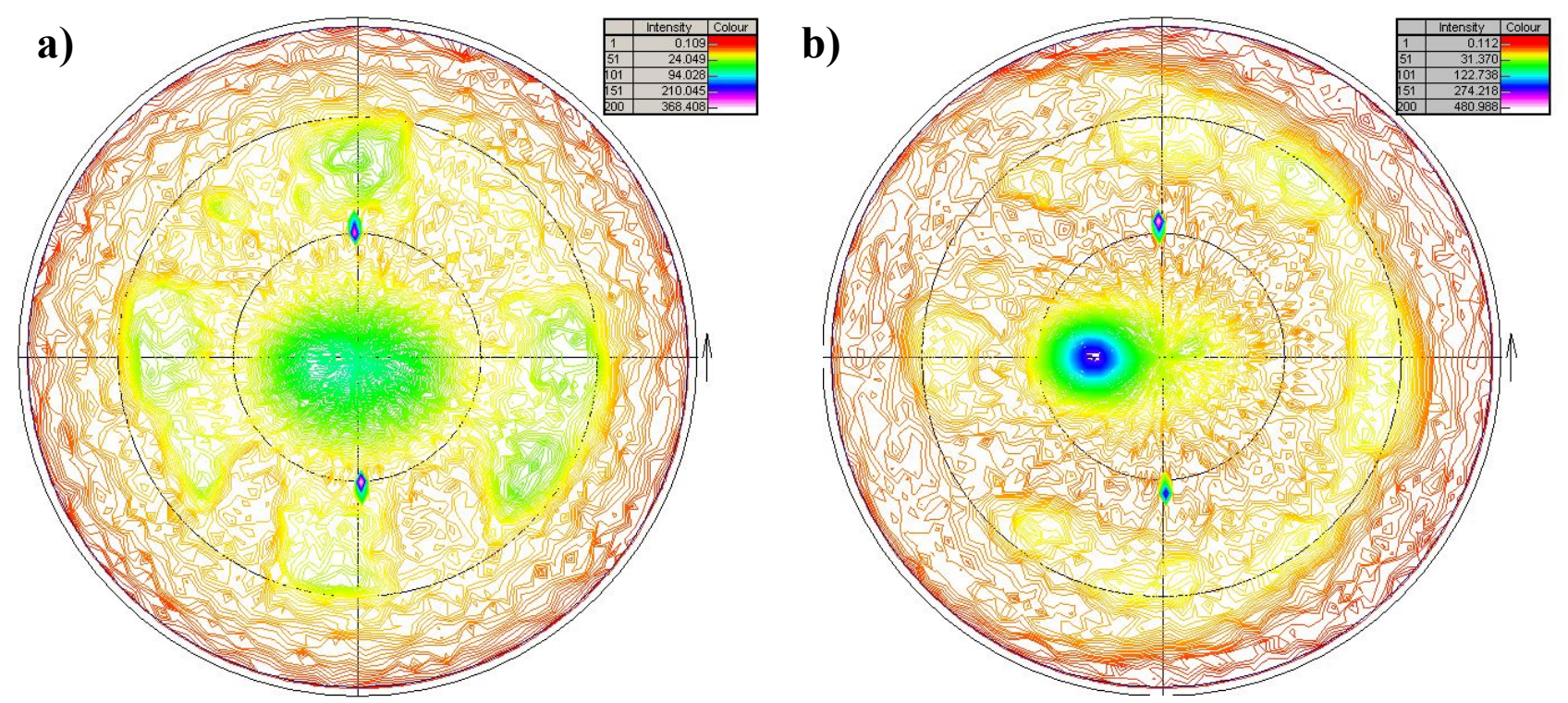

Abbildung 152: Texturanalyse: (111)-Polfiguren eines 50x(2nm Eisen/10nm Silber)-Schichtsystems: a) horizontal beschichtet nach $60 \mathrm{~min}$ bei $450^{\circ} \mathrm{C}$ und b) vertikal beschichtet nach $60 \mathrm{~min}$ bei $400^{\circ} \mathrm{C}$.

Zusammenfassend lässt sich feststellen, dass sich die Ergebnisse der Texturuntersuchung der Eisen/Gold-Schichten durch klarere Signaturen auszeichnen, die Schichten also größere Intensitäten sowie schärfere Reflexe bzw. ringförmige Intensitätsmaxima zeigen. Insbesondere bei Eisen/GoldSchichtsystemen auf facettiertem $\mathrm{Al}_{2} \mathrm{O}_{3}$ konnte gezeigt werden, dass (111)-orientierte Körner auf den Facettenseiten auf Kosten stark gestörter Bereiche auf den Facettenkämmen wachsen.

Trotz gleicher Dauer und höherer Endtemperatur der Temperung bedingt durch die höhere Zerfallstemperatur, ist das Gefüge des Schichtsystems Eisen/Silber weniger verändert als das Gefüge des Schichtsystems Eisen/Gold. Die Oberflächenmorphologie ist inhomogener und die Facettenseiten sind in der projizierenden Darstellung des SEM nicht unterscheidbar. Hingegen konnte durch eine 60minütige Temperung des Eisen/Gold-Systems bei $370^{\circ} \mathrm{C}$ eine Gefügetrennung auch an den Facettenkämmen beobachtet werden. Diese Gefügetrennung bestätigt die Beobachtungen der Untersuchungen mittels Röntgenbeugung. Sowohl Texturmessungen als auch in-situ Röntgen- 
heizmessungen zeigen für Substrate, die bei der Beschichtung eine vertikale Ausrichtung der Facetten aufwiesen, eine Reduktion der in Richtung der Gesamtsubstratnormalen (111)-orientierten Goldkörner durch die Temperung. Die SEM-Analyse zeigt, dass dies sogar deutlich sichtbare Veränderungen an der Oberfläche in Form von Trennungen oberhalb der Facettenkämme hinterlässt. TEM-Querschnittsanalysen an getemperten Eisen/Silber-Schichtsystemen auf facettiertem Substrat konnten durch zahlreiche Artefakte (wahrscheinlich durch präparative Probleme) kein vollständiges Bild von der Mikrostruktur, insbesondere von der Position der Eisenpartikel liefern. 


\section{Zusammenfassung}

Ziel dieser Arbeit war es, Selbstorganisationseffekte auszunutzen, um einerseits Substrate durch Oberflächenfacettierung im Nanometerbereich periodisch zu strukturieren und andererseits diese Anordnung in aufgebrachte dünne Filme und Schichtsysteme zu übertragen. Durch weitere nachfolgende thermisch induzierte Gestaltumwandlungen können damit auf technisch einfache Weise lineare Anordnungen metallischer und magnetischer Nanopartikel auf Oberflächen oder sogar im Volumen erzeugt werden.

Um diese gezielt beeinflusste Selbstordnung in den verschiedenen Dimensionen zu erreichen, wurde mit der Oberflächenfacettierung von $(10 \overline{1} 0)-\mathrm{Al}_{2} \mathrm{O}_{3}$ ein Prozess genutzt, der auf einfache Weise die thermodynamischen Eigenschaften der äußeren Kristallform und die elastische Wechselwirkung von Facettenlinien ausnutzt, um eine im Nanometerbereich periodisch strukturierte Oberfläche zu erzeugen. Analysen mittels AFM, aber auch Querschnittsuntersuchungen mittels TEM haben es ermöglicht, die unterschiedlichen Stadien der Facettierung zu beobachten. Nachdem Höhenfluktuationen an verschiedenen Stellen auf der Oberfläche Keime für die Facettenbildung darstellen, wachsen die Facetten mit parallelen Facettenlinien in [1 $\overline{2} 10]$-Richtung. Dabei bildet sich schnell die (1011) -Kristallfläche als eine der beiden Facettenseiten aus, während die andere Seite durch Aufstauen von kleineren Facetten gebildet wird und auch im Endzustand als eine nanofacettierte Facettenseite mit hohen ( $10 \overline{1} \overline{2})$-Flächenanteilen vorliegt. Durch Zusammenwachsen verschiedener Facettierungsdomänen gefolgt von Ausheilprozessen bildet sich schließlich nach $24 \mathrm{~h}$ bei $1550^{\circ} \mathrm{C}$ eine symmetrische, spitzdachartig rekonstruierte, periodische Oberfläche, deren Facettenseiten einen Winkel von $\sim 18^{\circ}$ zur ursprünglichen Oberfläche haben. Die laterale Periodizität, die durch die elastische Wechselwirkung der Facettenkämme und -täler im Wesentlichen über Materialkonstanten des Substrats bestimmt ist, beträgt $\sim 320 \mathrm{~nm}$, während die vertikale Amplitude auf $\sim 50 \mathrm{~nm}$ bestimmt werden konnte. Betrachtungen zur Thermodynamik und Kinetik vervollständigen das Verständnis des Facettierungsprozesses und zeigen weitere Beeinflussungsmöglichkeiten der Periodizität auf.

Diese Periodizität des Substrats gibt den Abstand von Drähten vor, die durch Schattendeposition unter flachem Winkel $\left(\sim 3^{\circ}\right)$ auf eine der beiden Facettenseiten aufgebracht werden konnten. Die Oberflächendiffusion des deponierten Materials auf den Facetten ist dabei entscheidend für das Wachstum und bestimmt, ob erfolgreich Nanodrähte erzielt werden können. Durch oberflächensensitive Verfahren, wie SEM-, AFM- und MFM-Analysen konnte gezeigt werden, dass bei hinreichend geringer Schichtdicke $<20 \mathrm{~nm}$ Nanodrähte der ferromagnetisch ordnenden Materialien Eisen und Kobalt mit Breiten im Bereich der Facettenseitenbreite von 160nm erfolgreich hergestellt werden konnten. Die Länge der Drähte ist dabei nur durch Fehler in der Facettierung begrenzt, deren mittlerer Abstand aus SEM-Untersuchungen auf ca. $100 \mu \mathrm{m}$ abgeschätzt werden konnte. Das Ummagnetisierungsverhalten dieser Anordnungen von Nanodrähten unterscheidet sich wesentlich von dem vergleichbarer dünner Schichten. Die Form der Drähte führt zu einer uniaxialen Anisotropie. Ein scharfer Übergang und die sprunghafte Änderung der Magnetisierungsrichtung beim Koerzitivfeld von $\mathrm{H}_{\mathrm{c}}=383 \pm 5$ Oe deutet auf eine Kopplung der Eisendrähte hin.

Neben der periodischen Anordnung der Drähte senkrecht zur Facettierungsrichtung konnte der thermische Zerfall der Drähte erfolgreich genutzt werden, um diese in Reihen von separierten, magnetischen Partikeln zu überführen. Durch in-situ Leitwertmessungen während des Anlassens konnte mit $550^{\circ}-600^{\circ} \mathrm{C}$ der für die selbstordnende Gestaltänderung relevante Temperaturbereich festgelegt werden. SEM- und AFM-Untersuchungen konnten zeigen, dass dem Zerfall eine mit der Temperatur steigende Aufrauung der Oberfläche vorausgeht. Die Drahtanordnungen beider untersuchten ferromagnetischen Systeme, Eisen und Kobalt, eignen sich für die Gestaltänderung. Sowohl die Partikelgröße, als auch die Separationsdistanz zeigen eine Proportionalität zur lokalen 
Facettenseitenbreite und können somit auch über die Schichtdicke der präparierten Drähte beeinflusst werden. Allerdings konnten nur bei Schichtdicken $<20 \mathrm{~nm}$ vollständig separierte Partikel durch den thermisch induzierten Zerfall erzeugt werden. Mit einem Durchmesser von 20 bis zu $\sim 160 \mathrm{~nm}$ (maximale Facettenseitenbreite) liegen in beiden Systemen runde Partikel vor. Größere, elliptisch in Facettierungsrichtung verformte Partikel zeigen große Halbachsen bis zu 350nm bei typischen Höhen von 40-70nm. Analysen remanenter Zustände nach Magnetisierung in Feldern unterschiedlicher Orientierung mittels MFM zeigen zum einen die Abhängigkeit der leichten Magnetisierungsrichtung von der Form, zum anderen, dass bei speziellen Größen und Abständen magnetostatische Kopplung zu einer gleichen Ausrichtung der Magnetisierung direkt benachbarter Partikel führen kann. Das Verschwinden der uniaxialen Formanisotropie bestätigt den Zerfall der Drähte. Die weitgehend winkelunabhängige Hysterese zeigt, dass weder in Facettierungsrichtung noch senkrecht dazu eine besonders ausgeprägte, magnetische Kopplung der Partikel vorliegt.

Um zu klären, ob die Periodizität der Unterlage in die Mikrostruktur der Schichtsysteme übertragbar ist, wurden Schichtsysteme der nicht mischenden Komponenten Eisen/Silber, Eisen/Gold und Kohlenstoff/Kobalt auf die facettierten Substrate deponiert und das Wachstum, die Mikrostruktur, das Gefüge und der Aufbau detaillierte charakterisiert. In-situ Leitfähigkeitsmessungen konnten zeigen, dass zunächst ein Volmer-Weber-Wachstum des Eisens auf dem $\mathrm{AbO}_{3}$-Substrat erfolgt. Die gegenüber den Volumenwerten um eine Größenordnung reduzierte Leitfähigkeit lässt auf das Vorliegen einer Vielzahl von mikrostrukturellen Defekten, wie z.B. Korngrenzen insbesondere parallel zur Schichtnormalen schließen. Da durch TAP-Messungen sowohl eine Segregation von Eisen in Silberkorngrenzen als auch eine starke Durchmischung der Grenzflächen ausgeschlossen werden konnte, wird die beobachtete Absenkung der Leitfähigkeit durch jede Eisenlage durch die starke Coulombstreuung der Leitungselektronen an Eisenatomen auf der Oberfläche erklärt. AFMund TEM-Querschnittsanalysen zeigen, dass die Oberflächenmorphologie des Substrats auch über große Gesamtschichtdicken von $600 \mathrm{~nm}$ hinweg an der Oberseite erhalten bleibt, vertikale Amplitude und Periodizitätslänge bleiben unverändert. Texturanalysen und röntgendiffraktometrische Messungen zeigen eine (111)-Drahttextur der Edelmetallkomponenten auf den Facettenseiten. Schärfere Reflexe und eine ausgeprägtere Übergitterstruktur in Großwinkeldiffraktogrammen weisen auf ein kohärenteres Wachstum im Schichtsystem Eisen/Gold gegenüber Eisen/Silber hin. Detaillierte TEM-Querschnittsanalysen der Schichtpakete belegen, dass die Facetten des Substrats wesentlichen Einfluss auf die Mikrostruktur in den Schichtsystemen haben und zeigen Säulen gleicher kristallographischer Orientierung den Ergebnissen der Texturmessungen entsprechend auf den Facettenseiten. Am amorphen Kohlenstoff/Kobalt-System, das sehr glatte Grenzflächen zeigt, konnten beispielhaft die Auswirkungen verschiedener Substratmerkmale auf das Wachstum analysiert werden. Während auf der glatten (10 $\overline{1} 1)$-Facettenseite ideal parallele Verläufe der inneren Grenzflächen zu beobachten waren, zeigte sich die nanofacettierte Gegenseite als Ausgangspunkt für Störungen, die zu kumulativen Modulationen der Grenzflächen mit der Lagenzahl führten. Zwei ausgezeichnete Bereiche sind über den Facettenkämmen und den -tälern gegeben. Während an den Kämmen ein keilförmiger, radialer Grenzflächenverlauf die Fehlorientierung über die Schichtdicke ausgleicht, ist oberhalb der Täler eine sehr schmale Störungszone erkennbar. Mittels HRTEM-Untersuchungen konnte am kristallinen System Eisen/Silber in diesen Bereichen ein sehr stark gestörtes, von vielen Korngrenzen dominiertes Gefüge identifiziert werden. Schon auf glatten Substraten konnte gezeigt werden, dass solche Defektbereiche bevorzugte Nukleationszentren für die Anlagerung von Nanopartikeln sind, die als Folge einer thermisch induzierten Gestaltumwandlung dieser entmischenden Schichtsysteme entstehen [Her 99], [Tro 00].

Leitfähigkeitsmessungen während der Temperbehandlungen der Schichtsysteme zeigen für Eisen/Silber eine Reduktion der Umwandlungstemperatur um mindestens 35K durch die nanostrukturierte Unterlage, während im Eisen/Gold-System der ohnehin niedrigere Umwandlungspunkt von $\sim 320^{\circ} \mathrm{C}$ nicht weiter reduziert wird. Durch den Aufbau einer neuen Vakuumkammer zur 
temperaturabhängigen Röntgendiffraktometrie am Hamburger Synchrotronstrahlungslabor (HASYLAB) war die Analyse der Gestaltumwandlung auch von Eisen/Silber-Schichtpaketen sehr geringer Reflektivität möglich. Zerfallstemperaturen, die durch den Verlust der Übergitterkohärenz bestimmt wurden, bestätigen die Absenkung dieser charakteristischen Temperatur für Eisen/Silberund die Konstanz für Eisen/Gold-Schichtsysteme. Die detaillierten Messungen liefern mit gegensätzlichen Spannungszuständen der Edelmetalle in den beiden Systemen außerdem einen Hinweis für die beobachteten Unterschiede. Gefüge- und Texturuntersuchungen nach dem Anlassen zeigen insbesondere im System Eisen/Gold, dass die Facettenkämme die Mikrostruktur des aufgebrachten Schichtsystems verändern, so dass nach einer Temperbehandlung von einer Stunde sogar eine Gefügetrennung oberhalb Facettenkämme an der Oberfläche des Schichtsystems sichtbar wird.

Somit ist es gelungen, die Substrate durch elastische Wechselwirkung von Facettenkämmen und -tälern auf Nanometer-Skala periodisch zu strukturieren, diese Facetten für die Herstellung von Nanodrähten durch Schattendeposition zu nutzen und deren technisch einfache, selbstorganisierte Umformung in lineare Anordnungen von metallischen Nanopartikeln zu erreichen, sowie die magnetischen Eigenschaften der verschiedenen Gestaltformen zu analysieren. Der Transfer der Periodizität der Substrate in Vielfachschichten konnte in Form von Störungszonen erzielt und die mikrostrukturellen Veränderungen in den Schichten umfassend charakterisiert werden. Damit erscheint auch die gezielte Anordnung der magnetischen Partikel im Volumen durch den thermisch induzierten Zerfall dieser Schichtsysteme möglich. 


\section{Literaturverzeichnis}

Abe 97: $\quad$ L. Abelmann, C. Lodder, Thin Solid Films 305 1-2 (1997) 1.

Aha 58: $\quad$ A. Aharoni, S. Shtrikman, Phys. Rev. 109 (1958) 1522.

All 86: G. L. Allen, R. A. Bayles, W. W. Gile, W. A. Jesser, Thin Solid Films 1442 (1986) 297.

Ami 04: S. Amirthapandian, B. K. Panigrahi, A. K. Srivastava, S. Dhara, A. Gupta, J. Appl. Phys. 9510 (2004) 5295.

Atk 88: $\quad$ A. Atkinson, Solid State Ionics 28-30 2 (1988) 1377.

Azz 77: $\quad$ R. M. A. Azzam, N. M. Bashara, Ellipsometry and polarized light North-Holland Publ. Co. Amsterdam (1977).

Bek 95: $\quad$ D.L. Beke, Y. Kaganovskii, Mat. Sci. Eng. B Solid. 32 (1995) 185.

Bia 02: L. Del Bianco, D. Fiorani, A. M. Testa E. Bonetti, L. Savini, S. Signoretti, Phys. Rev. B 66 (2002) 174418.

Bir 03: $\quad$ L. P. Biró, Z. Bálint, K. Kertész, Z. Vértesy, G. I. Márk, Z. E. Horváth, Phys. Rev. E 67 (2003) 21907.

Blu 93: $\quad$ S. J. Blundell, M. Gester, J. A. C. Bland, C. Daboo, E. Gu, M. J. Baird, J. Appl. Phys. 7310 (1993) 5948

Bol 04: $\quad$ A. P. Boltaev, N. A. Penin, A. O. Pogosov, F. A. Pudonin, JETP 994 (2004) 827.

Bor 02a: C.Borchers, P.Troche, C.Herweg, J.Hoffmann, J. Mat. Sci. 37 (2002) 791.

Bul 04: $\quad$ H. Bulou, F. Scheurer, P. Ohresser, A. Barbier, S. Stanescu, C. Quirós, Phys. Rev. B 69 (2004) 155413.

But 95: $\quad$ H.-J Butt, B. Gerharz, Langmuir 11 (1995) 4735.

Car 02: J. Carrey, J.-L. Maurice, Phys. Rev. B 65 (2002) 205401.

Che 97: K. Kuo-Fang Chen, Diplomarbeit, Institut für Metallphysik Universität Göttingen (1997).

Cul 67: B. D. Cullity, Elements of X-ray Diffraction Addison-Wesley Publishing Company (1967).

Dax 03: W. Dax, N. Drozd, W.D. Gläser, Tabellenbuch der Metalltechik Handwerk und Technik (2003).

Dzi 00: J. Dzick, Dissertation, Institut für Materialphysik Universität Göttingen (2000).

Edw 82: $\quad$ S. F. Edwards, D.R. Wilkinson, Proc. R. Soc. London, Ser. A 381 (1982) 17. 
Elm 94: $\quad$ H. J. Elmers, J. Hauschild, H. Höchle, U. Gradmann, H. Bethge, D. Heuer, Phys. Rev. Lett. 73 (1994) 898.

Fra 99: S. Franzka, Dissertation, Institut für Keramik im Maschienenbau Universität Karlsruhe (1999).

Fre 57: $\quad$ E. H. Frei, S. Shtrikman, D. Treves, Phys. Rev. 1063 (1957) 446.

Fre 87: H. Frey, G. Kienel, Dünnschicht Technologie VDI-Verlag GmbH (1987).

Fuc 38: $\quad$ K. Fuchs, Proc. Camb. Phil. Soc 34 (1938) 100.

Gie 03: $\quad$ F. J. Giessibl, Rev. Mod. Phys. 75 (2003) 949.

Gol 02: M. Golosovsky, Y. Saado, D. Davidov, Phys. Rev. E 65 (2002) 061405.

Gre 66: $\quad$ R. F. Greene, Phys. Rev. 1412 (1966) 687.

Gre 66a: $\quad$ R. F. Greene, R. W. O'Donnell, Phys. Rev. 147 (1966) 599.

Haa 94: $\quad$ P. Haasen, Physikalische Metallkunde Springer Berlin (1994).

Har 80: W. A. Harrison, Electronic Structure and the Properties of Solids W. H. Freeman and Company Stanford (1980).

Har 88: $\quad$ K. Hara, M. Kamiya, T. Hashimoto,K. Okamoto, H. Fujiwara, J. Magn. Magn. Mater. 732 (1988) 161.

Har 94: U. Hartmann, Adv. Electr. Electr. Phys. 87 (1994) 49.

Har 96: F. Hartung, Diplomarbeit, Institut für Metallphysik Universität Göttingen (1996).

Hat 01: $\quad$ A. Hatzor, P. S. Weiss, Science 2919 (2001) 1019.

Hef 97: J. R. Heffelfinger, Carter, C. B., Surf. Sci. 389 (1997) 188.

Hen 94: M. Henzler, W. Göpel, Oberflächenphysik des Festkörpers Teubner Stuttgart (1994).

Her 02: C. Herweg, S. Dreyer, P. Troche, J. Hoffmann, S. Sievers, C. Lang, H. C. Freyhardt, Mat. Res. Soc. Symp. Proc. 707 (2002) W6.35.

Her 99: C. Herweg, Diplomarbeit, Institut für Materialphysik Universität Göttingen (1999).

Heu 98: $\quad$ A. H. Heuer, K. P. Lagerlof, J. Castaing, Phil. Mag. A 783 (1998) 747.

Him 99: $\quad$ F. J. Himpsel, J. Phys.: Condens. Matter 11 (1999) 9483.

Hof 02: $\quad$ H. Hofmeister, S. A. Nepijko, D. N. Ievlev, W. Schulz, G. Ertl, J. Crys. Growth 234 (2002) 773.

Hof 81: H. Hoffmann, J. Vancea, Thin Solid Films 852 (1981) 147.

Hof 91: J. Hoffmann, Dissertation, Institut für Metallphysik Universität Göttingen (1991). 
Jac 90: $\quad$ U. Jacob, J. Vancea, H. Hoffmann, Phys. Rev. B 4115 (1990) 11852.

Kah 00: S. Kahl, Diplomarbeit, Institut für Materialphysik Universität Göttingen (2000).

Kar 86: $\quad$ M. Kardar, G. Parisi, Y.C. Zhang, Phys. Rev. Lett. 569 (1986) 889.

Kas 03: $\quad$ G. Kästle, H.-G. Boyen, F. Weigl, G. Lengl, T. Herzog, P. Ziemann, S. Rieth, Adv. Func. Mat. 1311 (2003) 863.

Kau 03: L.O. Kautschor, Dissertation, Institut für Materialphysik Universität Göttingen (2003).

Kel 91: $\quad$ D. Keller, Surf. Sci. 253 1-3 (1991) 353.

Kes 03: $\quad$ M. A. Kessler, B. T. Werner, Science 299 (2003) 380.

Klu 03: C. Kluthe, Dissertation, Institut für Materialphysik Universität Göttingen (2003).

Kre 84: $\quad$ H.U. Krebs, Dissertation, Institut für Metallphysik Universität Göttingen (1984).

Kro 57: $\quad$ M.L. Kronberg, Acta Met. 5 (1957) 507.

Kum 96: M. Kume, Atsushi Maeda, Toshio Tanuma, Kazuhiko Kuroki, J. Appl. Phys. 798 (1996) 6402.

Lai 95: $\quad$ B. M. Lairson, A. P. Payne, S. Brennan, N. M. Rensing, B. J. Daniels, B. M, Appl. Phys. 787 (1995) 4449.

Lan 82: $\quad$ Landolt-Börnstein - Group III, Electric Transport Phenomena Springer Berlin Heidelberg New York (1982).

Lan 02: C. Lang, Dissertation, Institut für Materialphysik Universität Göttingen (2002).

Lan 57: $\quad$ R. Landauer, IBM J. Res. Dev. 1 (1957) 223.

Lan 91: $\quad$ Landolt-Börnstein - Group IV, Physical Chemistry Al-O Springer Berlin Heidelberg New York (1991).

Liu 93: $\quad$ F. Liu, Horia Metiu, Phys. Rev. B 48 (1993) 5808.

Loc 89: J.-P. Locquet, D. Neerinck, L. Stockman, Y. Bruynseraede, Phys. Rev. B 3918 (1989) 13338

Mad 94: $\quad$ I. Manassidis, M.J. Gillan, J. Am. Ceram. Soc. 77 (1994) 335.

Mai 02: $\quad$ S. A. Maier, Mark L. Brongersma, Pieter G. Kik, Harry A. Atwater, Phys. Rev. B 65 (2002) 193408.

Mar 80: $\quad$ V. I. Marchenko, A. Ya. Parshin, Sov. Phys. JETP 52 (1980) 129.

Mar 81: $\quad$ V. I. Marchenko, Sov.Phys. JETP 54 (1981) 605.

Mat 93: $\quad$ G. Matteucci, M. Muccini, U. Hartmann, Appl. Phys. Lett. 6215 (1993) 1839.

May 70: $\quad$ A. F. Mayadas, M. Shatzkes, Phys. Rev. B 1 (1970) 1382. 
Mei 56: $\quad$ W. H. Meiklejohn, C. P. Bean, Phys. Rev. 1025 (1956) 1413.

Men 97: $\quad$ E. Mentz, D. Weiss, J. E. Ortega, A. Bauer, G. Kaindl, J. Appl. Phys. 82 (1997) 482.

Men 99: $\quad$ E. Mentz, A. Bauer, T. Günther, G. Kaindl, Phys. Rev. B 60 (1999) 7379.

Mey 03: G. Meyer, Dissertation, Freie Universität Berlin (2003).

Mey 96: J. A. Meyer, I. D. Baikie, E. Kopatzki, R. J. Behm, Surf. sci. lett. 365 (1996) L647.

Mil 96: M. K. Miller, A. Cereso, M. G. Hetherington und G. D. W. Smith, Atom Probe Field Ion Microscopy, Clarendon Press, Oxford (1996).

Mor 01: C. Mohr, M. Dubiel, H. Hofmeister, J. Phys. 13 (2001) 525.

Mul 61: $\quad$ W. W. Mullins, Phil. Mag. 6 (1961) 1313.

Mun 02: $\quad$ R. C. Munoz, R. Finge, C. Arenas, G. Kremer, L. Morag, Phys. Rev. B. 66 (2002) 205401.

Mün 97: M. Münzenberg, Diplomarbeit, I. Physikalisches Institut Universität Göttingen (1997).

Neu 62: $\quad$ C. A. Neugebauer, M. B. Webb, J. Appl. Phys. 331 (1962) 74.

Nic 66: $\quad$ F. A. Nichols, J. Appl. Phys. 377 (1966) 2805.

Odi 94: ～C. Odin, J. P. Aimé, Z. El Kaakour, T. Bouhacina, Surf. Sci. 3173 (1994) 321.

Oep 89: $\quad$ H. P. Oepen, J. Kirschner, Phys. Rev. Lett. 627 (1989) 819.

Ost 03: J. Oster, M. Kallmayer, L. Wiehl, H. J. Elmers, H. Adrian, M. Huth, submitted to Phys. Rev. B (2003).

Ozc 94: J. S. Ozcomert, W. W. Pai, N. C. Bartelt, J. E. Reutt-Robey, Phys. Rev. Lett. 72 (1994) 258.

Pau 58: $\quad$ L.J. van der Pauw, Philips Res. Rep. 131 (1958) 1.

Pha 91: R. J. Phaneuf, N. C. Bartelt, Ellen D. Williams, W. Swiech, E. Bauer, Phys. Rev. Lett. 67 (1991) 2986.

Pim 98: A. Pimpinelli, J. Villain, Physics of Crystal Growth Cambridge University Press (1998).

Pod 80: $\quad$ R. Podloucky, R. Zeller, P. H. Dederichs, Phys. Rev. B 22 (1980) 5777.

Pre 04: $\quad$ G. Prévot, B. Croset, Physical Review Letters 92 (2004) 256104.

Ram 00: $\quad$ S. Ramamurthy, H. Schmalzried, C. B. Carter, Phil. Mag. A 801 (2000) 2651.

Ram 98: K. A. Ramirez-Aguila, K. L. Rowlen, Langmuir 14 (1998) 2562.

Res 97: L. Ressier, A. Schuhl, F. Nguyen Van Dau, K. Postava, M. Goiran, J. P. Peyr, J. Appl. Phys. 81 (1997) 5464. 
Ros 01: C. A. Ross, Annual Review of Materials Research 31 (2001) 203.

Ros 02: C. A. Ross, S. Haratani, F. J. Castaño, Y. Hao, M. Hwang, M. Shima, J. Y., J. Appl. Phys. 9110 (2002) 6848.

Roy 92: $\quad$ W. Van Roy, J. De Boeck, G. Borghs, Appl. Phys. Lett. 6125 (1992) 3056.

Roy 93: W. Van Roy, E. L. Carpi, M. Van Hove, A. Van Esch, R. Bogaerts, J. De Boeck, J. Magn. Magn. Mater. 121 (1993) 197.

Saj 87: $\quad$ P. Sajgalik, Z. Panek, M. Uhrik, J. Mat. Sci. 2212 (1987) 4460.

Sch 04: C. Schug,Vortrag beim 191. PTB-Seminar am 11. Februar 2004.

Sch 92: $\quad$ M. A. Schildbach, A. V. Hamza, Phys. Rev. B 45 (1992) 6197.

Shc 95: V. A. Shchukin, A. I. Borovkov, N. N. Ledentsov, D. Bimberg, Phys. Rev. B 5115 (1995) 10104.

Shc 99: $\quad$ V. A. Shchukin, D. Bimberg, Rev. Mod. Phys. 71 (1999) 1125.

She 94: C. Shearwood, S. J. Blundell, M. J. Baird, J. A. C. Bland, M. Gester, H. Ah, J. Appl. Phys. 7510 (1994) 5249.

Shi 01: J. Shi, M. Azumi, O. Nittono, Appl. Phys. A. 73 (2001) 215.

Shi 04: $\quad$ S. Shiraki, Hideki Fujisawa, Masashi Nantoh, Maki Kawai, Appl. Surf. Sci. in press (2004)

Sht 03: M. Shtaya (Suleiman), Dissertation, Institut für Materialphysik Universität Göttingen (2003).

Sim 96: $\quad$ P. Simon, J. Ihlemann, Appl. Phys. A. 63 (1996) 505.

Sof 67: $\quad$ S. B. Soffer, J. Appl. Phys. 384 (1967) 1710.

Son 52: $\quad$ E. H. Sondheimer, Adv. Phys. 1 (1952) 1.

Son 97: $\quad$ S. Song, Mirang Yoon, S. G. J. Mochrie, G. B. Stephenson, S. T. Milner, Surf. Sci. 372 1-3 (1997) 37.

Sor 03: T.G. Sorop, C. Untiedet, F. Luis, L. J. de Jongh, M. Kröll, M. Rasa, J. Appl. Phys. 9310 (2003) 7044.

Spr 98: $\quad$ G. Springholz, V. Holy, M. Pinczolits, G. Bauer, Science 282 (1998) 734.

Str 04: C. Streng, Dissertation, I. Physikalisches Institut Universität Göttingen (2004).

Str 38: $\quad$ I. N. Stranski, L. Krastanov, Sitzungsbericht Akad. Wissenschaft Wien 146 (1938) 797.

Str 92: J. A. Stroscio, D. T. Pierce, R. A. Dragoset, P. N. First, J. Vac. Sci. Tech. A, 104 (1992) 1981.

Stu 00: K. Sturm, Dissertation, Institut für Materialphysik Universität Göttingen (2000). 
Stu 01: $\quad$ K. Sturm, H. U. Krebs, J. Appl. Phys. 902 (2001) 1061.

Sug 01: A. Sugawara, IEEE Transactions on Magnetics 374 (2001) 2123.

Sug 97: $\quad$ A. Sugawara, T. Coyle, G. G. Membree, M. R. Scheinfein, Appl. Phys. Lett. 708 (1997) 1043.

Sug 97a: $\quad$ A. Sugawara, M. R. Scheinfein, Phys. Rev. B 56 (1997) R8499.

Sus 92: $\quad$ D. W. Susnitzky, C. B. Carter, J. Am. Ceram. Soc. 759 (1992) 2463.

Tak 83: Y. Takeno, Y. Iwama, J. Magn. Magn. Mater. 35 (1983) 293.

Tas 88: $\quad$ P. W. Tasker, Adv. Ceram. 10 (1988) 176.

Tei 00: $\quad$ C. Teichert, C. Hofer, K. Lyutovich, M. Bauer, E. Kasper, Thin Solid Films 380 (2000) 25.

Tei 02: C. Teichert, Phys. Rep. 365 (2002) 335.

Ter 96: J. Tersoff, C. Teichert, M. G. Lagally, Phys. Rev. Lett. 7610 (1996) 1675.

Tes 86: Z. Tesanovic, M. V. Jaric, S. Maekawa, Phys. Rev. Lett. 5721 (1986) 2760.

Thi 04: K. Thiele, Dissertation, Institut für Materialphysik Universität Göttingen (2004).

Tri 02: $\quad$ M. I. Trioni, H. Ishida, G. P. Brivio, Phys. Rev. B 65 (2002) 125413.

Tro 00: P. Troche, Dissertation, Institut für Materialphysik Universität Göttingen (2000).

Tro 96: P. Troche, Diplomarbeit, Institut für Metallphysik Universität Göttingen (1996).

Tso 94: $\quad$ A. Tsoga, P. Nikolopoulos, J. Am. Ceram. Soc. 774 (1994) 954.

Tso 96: A. Tsoga, D. Sotiropoulou, P. Nikolopoulos, Mat. Sci. Forum 207-209 (1996) 565.

Van 87: J. Vancea, G. Reiss, H. Hoffmann, Phys. Rev. B 35 (1987) 6435.

Vol 26: M. Volmer, A. Weber, Z. Phys. Chem. 119 (1926) 277.

Vri 94: J. Vrijmoeth, H. A. van der Vegt, J. A. Meyer, E. Vlieg, R. J. Behm, Phys. Rev. Lett 72 (1994) 3843.

War 69: B. E. Warren, x-ray diffraction Addison-Wesley Reading, Menlo Park, London, Don Mills (1969).

Was 98: E. F. Wassermann, M. Thielen, S. Kirsch, A. Pollmann, H. Weinforth, A. Carl, J. Appl. Phys. 833 (1998) 1753.

Wes 04: $\quad$ A. Westphalen, H. Zabel, K. Theis-Bröhl, Thin Solid Films 449 1-2 (2004) 207.

Whi 02: G. M. Whitesides, B. Grzybowski, Science 295 (2002) 2418. 
Wie 01: U. Wiedwald, M. Spasova, M. Farle, M. Hilgendorff, M. Giersig, J. Vac. Sci. Tech. A 194 (2001) 1773.

Wul 01: G. Wulff, Z. Kristallogr. Mineral. 34 (1901) 449.

Yos 04: $\quad$ M. Yoshino Y. Shinzato, M. Morinaga, Mat. Sci. Forum 449-452 (2004) 713.

Zil 94: $\quad$ H. Zillgen, B. Feldmann, M. Wuttig, Surf. Sci. 321 (1994) 32. 



\section{Danksagung}

Herrn Prof. Dr. H. C. Freyhardt danke ich für die Ermöglichung dieser Arbeit und die großen Freiräume, die er mir gelassen hat, eigene Ideen und Vorstellungen umzusetzen.

Mein besonderer Dank gilt den Herren Prof. Dr. H.-U. Krebs, Dr. J. Hoffmann und PD. Dr. C. Jooß, die diese Arbeit begleitet und durch viele Diskussionen, Anregungen und Korrekturvorschläge bereichert haben.

Den Mitgliedern der Sputtergruppe, Herrn Dr. P. Troche, Frau Dr. S. Sievers, Frau Dr. K. Herweg, Herrn Dr. L.-O. Kautschor, Frau Dr. C. Brandt, Herrn S. Dreyer und Herrn C. Thiele, aber auch den Mitgliedern der PLD-Gruppe, Herrn E. Süske, Herrn J. Faupel, Herrn T. Scharf, Herrn C. Fuhse, Herrn A. Meschede und Frau J. Röder danke ich für zahllose Diskussionen über Physik und anderes und die gute Atmosphäre.

Herrn PD Dr. M. Seibt danke ich für die Hilfe am CM200, als das 420er den Dienst versagte.

Herrn Prof. Dr. W. H.-G. Müller von der Fachhochschule Hildesheim/Holzminden/Göttingen danke ich für das Vertrauen und die Möglichkeit zur Nutzung des Rasterkraftmikroskops,- Herrn Prof. Dr. W. Felsch für die Möglichkeit zur Nutzung der MOKE-Apparatur und Frau PD Dr. A. Pundt und Herrn Dr. J. Bankmann für die Möglichkeit zur Durchführung von Messungen am HASYLAB.

Frau Dr. S. Sievers danke ich für die Unterstützung durch MFM- und SQUID-Messungen, Herrn Dr. C. Kluthe für die Hilfe bei den TAP-Analysen.

Frau K. Gehrke, Herrn L. Kirchhoff, Herrn M. Malchow und Herrn M. Hahn danke ich für die unproblematische, technische Unterstützung im Labor, bei den Sputteranlagen und diversen Präparations- und Analysegeräten.

Den Mitarbeitern des Instituts für Materialphysik und allen, die das Mittagessen, die Kaffeerunden und die Seminarnachbesprechung zu wichtigen Ereignissen mit vielen Diskussionen und einer motivierenden Atmosphäre haben werden lassen, danke ich ebenfalls sehr.

Den Mitarbeitern des Zentrums für Funktionswerkstoffe danke ich für die nette Unterstützung und Partnerschaft in der Zeit im Windausweg.

Meiner Familie und meinen Freunden danke ich besonders, da sie mich während der Arbeit unterstützt, ermutigt und begleitet haben. 


\section{Lebenslauf}

\section{Persönliche Daten}

Name:

Geburtsdatum:

Geburtsort:

Staatsangehörigkeit:

\section{Schulbildung}

$1980-1984$

$1984-1993$

16.06.1993

\section{Zivildienst}

$1993-1994$

\section{Studium}

WS 1994

05.07.1996

WS 1996 - SS 1997

WS 1998 - SS 1999

29.10.1999

SS 2000

WS 2002

\section{Beschäftigungen}

seit 1.12.1999
Carsten Herweg

27.06.1974

Opladen jetzt Leverkusen

deutsch
Grundschule in der Wasserkuhl/Neuboddenberg in Leverkusen Gymnasium im Schulzentrum Schlebusch Abitur
Aufnahme des Physikstudiums an der Universität Göttingen Vordiplomprüfung Physik

Studium der Physik an der Universität Uppsala, Schweden Hauptstudium Physik an der Universität Göttingen

Diplomarbeit bei Prof. Dr. H. C. Freyhardt am Institut für Materialphysik mit dem Thema:

GMR als Sonde zur Untersuchung thermisch induzierter Gestaltinstabilitäten magnetischer Vielfachschichten

Diplomprüfung Physik

Einschreibung in den Aufbaustudiengang Physik an der Universität Göttingen

Beginn der Dissertation in der Arbeitsgruppe von Herrn Prof. Dr. H.C. Freyhardt am Institut für Materialphysik

Umschreibung in den Promotionsstudiengang Physik an der Universität Göttingen

wissenschaftlicher Mitarbeiter des Instituts für Materialphysik der Universität Göttingen 